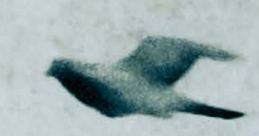

\title{
TRANSPORT NEAR SLIPPERY \\ INTERFACES
}

5.
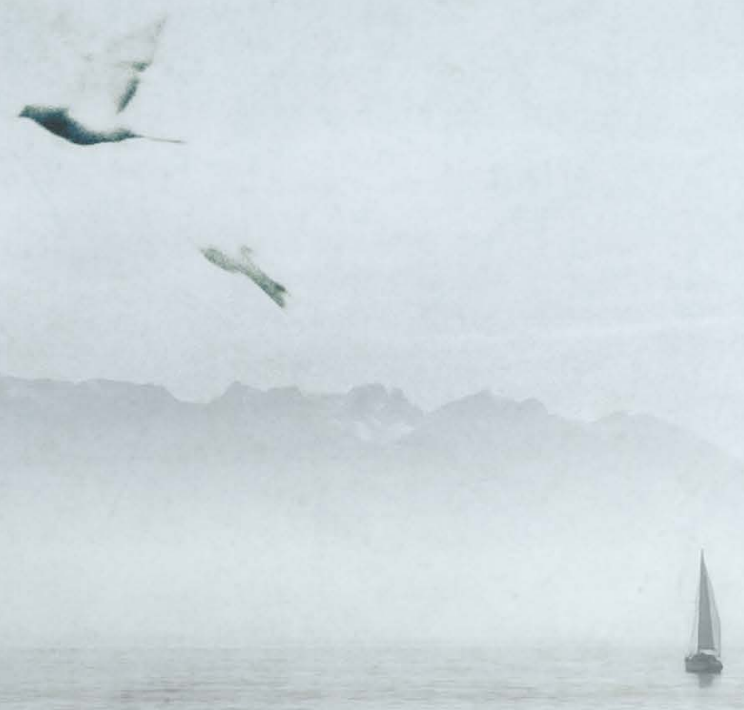

A. SANDER HAASE 



\section{DISSERTATION}

Transport near slippery interfaces

Transport nabij glibberige grensvlakken

by $A$. Sander Haase 


\section{Promotiecommissie}

Voorzitter Prof. dr. ir. J.W.M. Hilgenkamp Universiteit Twente

Promotor Prof. dr. ir. R.G.H. Lammertink Universiteit Twente

Copromotor Dr. J.A. Wood

Universiteit Twente

Overige leden Dr. C. Cottin-Bizonne

Prof. dr. J.C.T. Eijkel

Université Claude Bernard Lyon 1

Prof. dr. rer. nat. S. Hardt

Universiteit Twente

Prof. dr. ir. J.A.M. Kuipers

Technische Universität Darmstadt

Prof. dr. rer. nat. D. Lohse

Technische Universiteit Eindhoven

Universiteit Twente

This thesis is part of the TRAM-project

(transport at the microscopic interface),

funded by the European Research Council.

This work was performed at

Soft matter, Fluidics and Interfaces

$\mathrm{MESA}^{+}$Institute for Nanotechnology

Faculty of Science and Technology

University of Twente

P.O. Box 217

7500 AE Enschede

The Netherlands

Transport near slippery interfaces

ISBN: 978-90-365-4180-0

DOI: $10.3990 / 1.9789036541800$

URL: http://dx.doi.org/10.3990/1.9789036541800

Cover design by A. van de Maat and A.S. Haase

Typeset in $\mathrm{LAT}_{\mathrm{E}} \mathrm{X}$

Printed by Gildeprint

Copyright (C) 2016 by A.S. Haase 


\title{
TRANSPORT NEAR SLIPPERY INTERFACES
}

\section{PROEFSCHRIFT}

\author{
ter verkrijging van \\ de graad van doctor aan de Universiteit Twente, \\ op gezag van de rector magnificus, \\ prof. dr. H. Brinksma, \\ volgens besluit van het College voor Promoties \\ in het openbaar te verdedigen \\ op vrijdag 4 november 2016 om 14.45 uur
}

door

\section{Andries Sander Haase}

geboren op 21 februari 1988

te Deventer, Nederland 
Dit proefschrift is goedgekeurd door

Prof. dr. ir. R.G.H. Lammertink (promotor)

en

Dr. J.A. Wood (copromotor) 


Our little systems have their day; They have their day and cease to be: They are but broken lights of Thee, And Thou, O Lord, art more than they.

- In Memoriam A.H.H. by Alfred, Lord Tennyson 



\section{Contents}

Summary $\quad$ xv

Samenvatting $\quad$ xix

1 Transport near slippery interfaces: an introduction $\quad 1$

1.1 Slip or no-slip? . . . . . . . . . . . . . . . . . . . . . 2

1.1.1 A historical overview . . . . . . . . . . . . . 2

1.1.2 The slip boundary condition ............ 5

1.2 Momentum transport . . . . . . . . . . . . . 6

1.2.1 Renewed interest in the no-slip boundary condition . . . . . 6

1.2.2 Slippery smooth surfaces . . . . . . . . . . . . . 6

1.2.3 What causes wall slip? . . . . . . . . . . . . . . 9

1.2.4 Superhydrophobicity . . . . . . . . . . . . 10

1.2.5 Slippery superhydrophobic surfaces . . . . . . . . . . . 12

1.2.6 Analytical relationships . . . . . . . . . . . . . 13

1.2.7 Curved gas-liquid interfaces . . . . . . . . . . . . . 15

1.2.8 Shape, stability and contamination . . . . . . . . . 16

1.2 .9 Polymer solutions . . . . . . . . . . . . . . 17

1.3 Heat and mass transfer . . . . . . . . . . . . . . . . . . 18

1.3.1 Concentration polarisation ............ 18

1.3.2 A classical forced convection problem . . . . . . . . . 21

1.3.3 Increased convection near the wall . . . . . . . . . . . 22

1.3.4 Heat and mass transfer near superhydrophobic surfaces . . 23

1.3.5 Wall heterogeneity ................ 25

1.4 Charge transport near heterogeneous surfaces . . . . . . . . . 25

1.5 Utilising hydrodynamic slip and wall heterogeneity . . . . . . . . . 27

1.6 Thesis outline . . . . . . . . . . . . . . . . . . . . . . . 28

References ......................... 29

2 Controlling momentum transport over a heterogeneously slippery $\begin{array}{ll}\text { bubble mattress } & 43\end{array}$

2.1 Introduction . . . . . . . . . . . . . . . . . . . . . . 44

2.2 Materials and methods . . . . . . . . . . . . . . . . . . 45

2.2.1 Fabrication of microfluidic devices . . . . . . . . . 45 




2.2 .3 Numerical procedure . . . . . . . . . . . . . . . . 47

2.2.4 Calculation of effective slip length _ . . . . . . . . . 47

2.2.5 Calculation of friction factor . . . . . . . . . . . . 48

2.3 Results and discussion . . . . . . . . . . . . . . . . . 49

2.3 .1 Measuring the flow field . . . . . . . . . . . . . . . 49

$2.3 .2 \quad$ Effective wall slip . . . . . . . . . . . . . . . . 52

2.3.3 Comparison with analytical asymptotic solutions . . . . . 55

2.3.4 Drag reduction . . . . . . . . . . . . . . . . 56

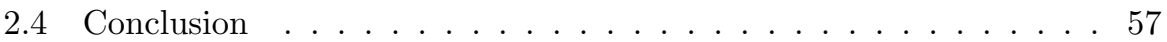

References . . . . . . . . . . . . . . . . . 58

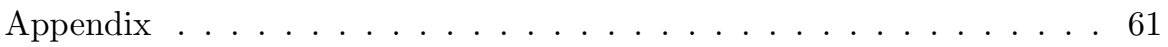

3 Why bumpy is better: the role of the dissipation distribution in slip flow over a bubble mattress $\quad \mathbf{6 5}$

3.1 Introduction . . . . . . . . . . . . . . . 66

3.2 Mathematical formulation . . . . . . . . . . . . . . 67

3.2 .1 Governing equations . . . . . . . . . . . . . . . . 67

3.2.2 Relating the effective slip length to viscous dissipation . . . 69

3.3 Results and discussion . . . . . . . . . . . . . . . . 71

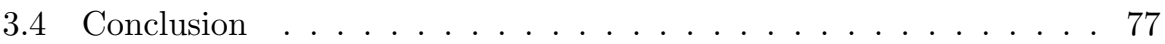

References . . . . . . . . . . . . . . . . 78

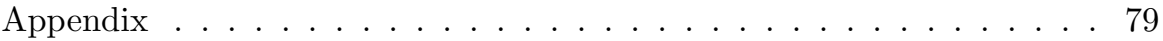

4 Inelastic non-Newtonian flow over heterogeneously slippery surfaces 83

4.1 Introduction . . . . . . . . . . . . . . . . . . . 84

4.2 Methodology . . . . . . . . . . . . . . . . . 87

4.2.1 Flow curves of xanthan gum solutions _ . . . . . . . 87

4.2 .2 Numerical method . . . . . . . . . . . . . . . . . 88 88

4.2.3 Determination of the effective slip length _ . . . . . . . 90

4.3 Limiting values for effective wall slip . . . . . . . . . . . . 91

4.4 Results and discussion . . . . . . . . . . . . . . . . . . 94

4.4 .1 Numerical results . . . . . . . . . . . . . . . . . . . . . . 94

4.4 .2 Predicting the enhancement . . . . . . . . . . . . . 997

4.4.3 Relationship to hydrodynamic development length . . . . . 100

4.5 Conclusion . . . . . . . . . . . . . . . . . . . 101

References . . . . . . . . . . . . . . . . . . . . 102

Appendix . . . . . . . . . . . . . . . . . . 106

5 Momentum and mass transport over a bubble mattress: the $\begin{array}{lr}\text { influence of interface geometry } & 109\end{array}$

5.1 Introduction . . . . . . . . . . . . . . . . . . 110 
5.2 Mathematical formulation . . . . . . . . . . . . . . . . . 112

5.2.1 Computational model . . . . . . . . . . . . . . . . . 112

5.2 .2 Reference models . . . . . . . . . . . . . . . . 115

5.3 Results and discussion . . . . . . . . . . . . . . . . 116

5.3.1 Momentum transport . . . . . . . . . . . . . 116

5.3 .2 Interfacial mass transport . . . . . . . . . . . . . . . . . . 119

5.4 Conclusions . . . . . . . . . . . . . . . . . . . . . 125

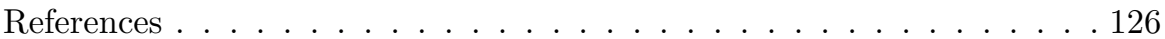

6 The Graetz-Nusselt problem extended to continuum flows with $\begin{array}{ll}\text { finite slip } & 131\end{array}$

6.1 Introduction . . . . . . . . . . . . . . . . . . . . 132

6.2 Mathematical model . . . . . . . . . . . . . . . . . . 134

6.2.1 Heat equation . . . . . . . . . . . . . . . . . 134

6.2.2 Nusselt number . . . . . . . . . . . . . . . . 135

6.2.3 Analytical expressions for thermally developing flow . . . . 135

6.2.4 Thermal and viscous boundary layer thickness . . . . . . 137

6.2.5 Numerical procedure . . . . . . . . . . . . . . . . . . 137

6.3 Results and discussion . . . . . . . . . . . . . . . . . 137

6.3.1 Nusselt profiles . . . . . . . . . . . . . . . . 137

6.3.2 On $\beta$ and its transition point . . . . . . . . . . . 139

6.3.3 On $N u_{\infty}$ and its transition point . . . . . . . . . . . . . . . 142

6.3.4 Employing wall slip to increase heat transfer . . . . . . . . 142

6.4 Conclusion . . . . . . . . . . . . . . . . . 143

References . . . . . . . . . . . . . . . . 144

Appendix . . . . . . . . . . . . . . . . 146

7 Heat and mass transfer over slippery, superhydrophobic surfaces 153

7.1 Introduction . . . . . . . . . . . . . . . . . . . . . . . . . . 154

7.2 Methodology . . . . . . . . . . . . . . 156

7.2.1 Governing equations . . . . . . . . . . . . . 156

7.2.2 Nusselt/Sherwood number . . . . . . . . . . . . . . . . . 158

7.2.3 Boundary conditions . . . . . . . . . . . . . . . . . 158

7.2 .4 Effective slip length . . . . . . . . . . . . . . . . . 159

7.3 Numerical approach . . . . . . . . . . . . . . . . . . . . 161

7.4 Results and discussion . . . . . . . . . . . . . . . . . . 162

7.4.1 Local Nusselt/Sherwood number . . . . . . . . . . . . . . . 162

7.4.2 Average Nusselt/Sherwood number . . . . . . . . . . . . . 167

7.4.3 The significance of axial diffusion - local Péclet number . . 169

7.4.4 Influence of axial diffusion on heat/mass transport . . . . . 171

7.5 Conclusion . . . . . . . . . . . . . . . . . . 173 


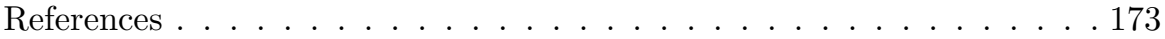

8 Desalination by electrodialysis using a stack of ion-selective $\begin{array}{lr}\text { hydrogels on a microfluidic device } & 179\end{array}$

8.1 Introduction . . . . . . . . . . . . . . . . . . . . . . 180

8.2 Experimental . . . . . . . . . . . . . . . 181

8.2.1 Microchip and hydrogel fabrication . . . . . . . . . . . 181

8.2.2 Characterisation of hydrogels . . . . . . . . . . . . . . 184

8.3 Results and discussion . . . . . . . . . . . . . . . . . . 186

8.3.1 Characterisation of hydrogels . . . . . . . . . . 186

8.3.2 Desalination - proof of principle experiments . . . . . . 189

8.4 Conclusion . . . . . . . . . . . . . . . . . . 195

References . . . . . . . . . . . . . . . . . 196

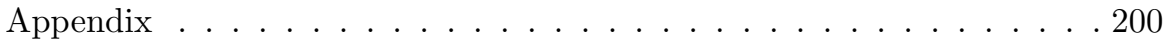

9 Conclusions and outlook 205

9.1 Conclusions . . . . . . . . . . . . . . . . . . . . . 205

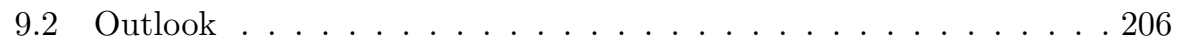

9.2.1 Enhanced transport . . . . . . . . . . . 206

9.2.2 Visco-elasticity and wall slip . . . . . . . . . . . . 207

9.2.3 Surface-driven flows . . . . . . . . . . . . . . . . 209

9.2.4 Electrokinetic transport and wall heterogeneity . . . . . . . 212

References . . . . . . . . . . . . . . . . . . . 212

$\begin{array}{lr}\text { Acknowledgements } & 219\end{array}$

$\begin{array}{ll}\text { About the author } & 223\end{array}$

$\begin{array}{lr}\text { Scientific output } & 225\end{array}$ 




\section{SUMMARY}

\section{Transport near slippery interfaces}

The phenomenon of wall slip can be utilised to increase advection in the direct vicinity of a wall. As this also affects the rate of transport at larger scales, slippery surfaces have a potentially important application in enhancing interfacial transport. The aim of this thesis is to obtain further insight into the transport phenomena near slippery and/or heterogeneous interfaces. A general introduction to this topic is given in chapter 1. First, an overview is given of the boundary conditions to apply at slippery surfaces. This is followed by a discussion of the various systems in which hydrodynamic slip can be encountered. Finally, the relationship between slippage and/or surface heterogeneity and interfacial transport is briefly discussed.

It has been predicted that for flow over a bubble mattress, which is a superhydrophobic surface consisting of an array of solid ridges and slippery bubbles positioned perpendicular to the flow direction, the amount of wall slip depends on the curvature of the gas-liquid interface. In chapter 2, we describe the experimental verification of this predicted dependency using micro-particle image velocimetry. The design of the glass-silicon microfluidic devices, containing channels of $50 \mu \mathrm{m}$ high and bubbles with a size of $20 \mu \mathrm{m}$, allowed active control of the bubble protrusion angle $\vartheta$ in the range of $-5^{\circ}<\vartheta<45^{\circ}$. We found good qualitative and quantitative agreement between the experimentally determined slip lengths, quantifying the amount of slippage, and the numerical predictions. Reinterpretation of the data in terms of the friction factor illustrates that the slip phenomena in such devices could be used to control hydrodynamic friction.

The experimental results show that wall slip is maximum for a protrusion angle in the range of $0^{\circ}<\vartheta<15^{\circ}$. The optimum angle depends on the system. Various numerical and analytical studies confirm that slippage is maximised for bubbles that somewhat protrude in the channel flow. In chapter 3 we answer the question of why this is the case by analytically and numerically analysing the spatial distribution of the viscous dissipation rate for varying protrusion angles. When this angle increases, the bubbles increasingly form an obstacle to the flow and reduce the effective channel height. This increases the dissipation in the bulk flow. Near the bubble corners, which form the contact points of the no-slip 
channel wall and the no-shear bubble surfaces, the dissipation rate decreases with increasing protrusion angle. An analytical expression, based on classical corner flow solutions, quantifies this. These two opposing effects explain why effective wall slip is maximised for a bubble mattress that is slightly bumpy.

In chapter 4, we extend our studies on momentum transport over heterogeneously slippery surfaces to inelastic non-Newtonian fluids, represented by shear-thinning aqueous xanthan gum solutions. Our simulation results reveal that wall slip can be increased significantly for flow over a bubble mattress when compared to Newtonian fluid flow. To observe this enhancement for a Carreau fluid, the system needs to be operated in the shear-thinning regime. We could predict the maximum slip enhancement analytically by deriving a theoretical limit for the effective slip length for flow of a power-law fluid over a surface containing transverse no-slip and no-shear regions. This limit is reached when these regions are much longer than the characteristic size of the system, allowing the fluid flow to adapt fully to the no-slip and no-shear conditions at the wall. The slip enhancement for shear-thinning liquids with respect to a Newtonian fluid is independent of the size of the no-slip/no-shear regions.

Wall slip increases fluid flow and therefore convection near the wall. As described in chapter 5, we investigated numerically the effect of a heterogeneously slippery bubble mattress on interfacial mass transfer. A fixed solute concentration was applied to the gas-liquid interfaces, whereas the solid wall in between the bubbles was impermeable. As such, the model formed a two-dimensional representation of a microporous membrane. Even though mass is exchanged only at the gas-liquid interfaces of a bubble mattress, our results predict a significant enhancement in mass transfer with respect to a non-slippery substrate having a uniform solute concentration. The enhancement is a strong function of the bubble protrusion angle, the coverage of the surface by bubbles and the flow conditions in the channel. Additionally, the enhancement vanishes when the bubbles become very small compared to the characteristic size of the channel. Since the amount of slip scales with bubble size, transport near small bubbles is dominated by diffusion. As a consequence, for small bubbles the porous and slippery bubble mattress can be considered as a fully solute-saturated, non-slippery surface.

To study the relationship between hydrodynamic slip and interfacial transport on a more general level, we extended the classical Graetz-Nusselt problem to continuum flows with homogeneous wall slip. This problem concerns heat or mass transfer between a hydrodynamically developed laminar flow and a tube having a constant wall temperature or concentration. As described in chapter 6, we derived an analytical expression for the Nusselt or Sherwood number in the developing regime that is valid for any value of the slip length. It reduces to the classical solutions for no-slip Hagen-Poiseuille flow and for no-shear plug flow in the respective asymptotic limits. The Nusselt and Sherwood numbers for partial 
wall slip gradually transition from the lower to the upper classical limit when increasing the slip length from zero to infinity. A physical interpretation of the results revealed that the heat/mass transfer mechanism in the developing regime depends on the velocity profile in the thermal/mass boundary layer. The relative significance of the slip velocity determines whether it resembles the transport mechanism for no-slip or for no-shear flow. Transport in the developed regime, where the thermal/mass boundary layer is larger than the tube radius and the Nusselt/Sherwood number is constant, depends on the velocity profile only.

Slip flow over superhydrophobic surfaces is highly heterogeneous, as those surfaces are characterised by a pattern of (in the ideal case) no-slip and no-shear regions. Chapter 7 concerns the extension of the Graetz-Nusselt problem to heat and mass transfer over heterogeneously slippery, superhydrophobic surfaces. The boundary conditions are different for heat and mass transfer. When describing heat transfer, due to differences in thermal conductivity, the solid no-slip wall has a constant wall temperature and the slippery gas-liquid interface can be treated as adiabatic. For mass transport, considering transport near a gas-filled porous membrane, the no-shear gas-liquid interface has a constant wall concentration and the solid wall is impermeable. Because only for mass transport the locations for hydrodynamic slip and mass exchange coincide, mass transfer is faster than thermal transport. Generally, thermal diffusion is much faster than mass diffusion. For that reason, axial conduction cannot be neglected when describing heat transfer in microscale systems. For mass transfer, axial diffusion only has a minor influence on the transport rate.

In chapter 8 we describe the development of a polydimethylsiloxane microfluidic electrodialysis platform containing a stack of alternatingly positively and negatively charged hydrogels. The wall is heterogeneous, containing regions that are impermeable. Under an electrical potential difference, ions are transported selectively through the anion- and cation-exchange hydrogels. This leads to either enrichment or depletion of the inflowing salt solutions. For sufficiently high voltages, we observed the formation of vortices, which enhances convective transport near the hydrogel interfaces in the overlimiting current regime. Our experimental electrodialysis platform allows visualisation of ion transport inside the hydrogels, which can be coupled to transport near the interfaces and in the bulk. This device can therefore contribute to a better understanding of the various transport phenomena related to electrodialysis.

The main conclusions of the work described in this thesis are provided in chapter 9, followed by some suggestions for future research on slippery surfaces and their use for enhancing interfacial transport. These include the application of superhydrophobic surfaces in large-scale processes containing microscale structures, the enhancement of surface-driven flows by hydrodynamic wall slip and the influence of visco-elastic fluids on slippage and the possible use thereof. 



\section{SAMENVATTING}

\section{Transport nabij glibberige grensvlakken}

Het verschijnsel van wandslip kan worden gebruikt om advectie in de directe nabijheid van een wand te vergroten. Aangezien dit eveneens de transportsnelheid op grotere schaal beïnvloedt, hebben glibberige oppervlakken een potentieel belangrijke toepassing in het versterken van grensvlaktransport. Het doel van dit proefschrift is om meer inzicht te verkrijgen in de transportverschijnselen nabij glibberige en/of heterogene oppervlakken. Hoofdstuk 1 vormt een algemene introductie op dit onderwerp. Allereerst wordt een overzicht gegeven van de randvoorwaarden die van toepassing zijn op glibberige oppervlakken. Vervolgens worden verschillende systemen besproken waarin sprake is van hydrodynamische slip. Tenslotte wordt kort de wisselwerking tussen slip en/of oppervlakteheterogeniteit en grensvlaktransport behandeld.

Men heeft voorspeld dat voor stroming over een bellenmatras de hoeveelheid wandslip afhangt van de kromming van het gas-vloeistof grensvlak. Een bellenmatras is een superhydrofoob oppervlak dat bestaat uit een reeks van vastestofrichels en glibberige (lucht)bellen die haaks op de stromingsrichting zijn gepositioneerd. In hoofdstuk 2 beschrijven wij de experimentele verificatie van deze voorspelde afhankelijkheid, gebruikmakend van micro-particle image velocimetry. Het ontwerp van het uit glas en silicium gefabriceerde microfluïdische platform, dat bestaat uit kanaaltjes met een hoogte van $50 \mu \mathrm{m}$ en gasbellen met een grootte van $20 \mu \mathrm{m}$, maakte het mogelijk om de protrusiehoek $\vartheta$ van de bellen actief te controleren in het bereik van $-5^{\circ}<\vartheta<45^{\circ}$. Wij vonden goede kwalitatieve en kwantitatieve overeenstemming tussen de experimenteel bepaalde sliplengten, die de hoeveelheid slip kwantificeren, en de numerieke voorspellingen. Herinterpretatie van deze data in termen van de frictiefactor illustreert dat de slipverschijnselen in dergelijke microchips zouden kunnen worden gebruikt om hydrodynamische frictie te controleren.

De experimentele resultaten laten zien dat wandslip maximaal is voor een protrusiehoek in het bereik van $0^{\circ}<\vartheta<15^{\circ}$. De optimale hoek hangt af van het systeem. Verschillende numerieke en analytische studies bevestigen dat slip wordt gemaximaliseerd wanneer de gasbellen iets uitsteken in de vloeistofstroming door het kanaal. In hoofdstuk 3 beantwoorden wij de vraag waarom dit het geval is 
door analytisch en numeriek de ruimtelijke verdeling van de visceuze dissipatiesnelheid te analyseren voor verschillende protrusiehoeken. De bellen vormen in toenemende mate een obstakel voor de stroming wanneer deze hoek toeneemt. Tevens reduceren ze de effectieve kanaalhoogte. Daardoor neemt de dissipatie in de stroming toe. Nabij de hoeken van de gasbellen, die de contactpunten vormen voor de niet-glibberige kanaalwand en de afschuifspanningsvrije bellen, neemt de dissipatie af wanneer de hoek toeneemt. Dit wordt gekwantificeerd door een analytische uitdrukking die gebaseerd is op klassieke analytische oplossingen voor stroming in een hoek. Deze beide tegenstrijdige effecten verklaren waarom effectieve wandslip maximaal is voor een enigszins bobbelige bellenmatras.

In hoofdstuk 4 breiden we ons onderzoek naar momentumtransport over heterogeen glibberige oppervlakken uit naar inelastische niet-Newtoniaanse vloeistoffen, voorgesteld door pseudoplastische oplossingen van xanthangom in water. De simulatieresultaten wijzen uit dat voor stroming over een bellenmatras, in vergelijking met Newtoniaanse vloeistoffen, wandslip significant kan worden versterkt. Om deze vermeerdering waar te nemen voor een Carreauvloeistof dient het systeem werkzaam te zijn in het pseudoplastische gebied. De maximale versterking in slip konden wij voorspellen door een theoretische limiet af te leiden voor effectieve slip in de stroming van een machtsfunctievloeistof over een oppervlak dat bestaat uit transversale niet-glibberige en afschuifspanningsvrije domeinen. Deze limiet wordt bereikt wanneer deze domeinen veel langer zijn dan de karakteristieke grootte van het systeem, waardoor de vloeistofstroming zich volledig aan kan passen aan de slipvrije en afschuifspanningsvrije condities aan de wand. De slipvermeerdering voor pseudoplastische vloeistoffen in vergelijking met Newtoniaanse vloeistoffen is echter onafhankelijk van de grootte van de slipvrije en afschuifspanningsvrije domeinen.

Wandslip versnelt vloeistofstroming en daarmee convectie nabij de wand. Zoals beschreven in hoofdstuk 5 hebben wij de effecten van een heterogeen glibberige bellenmatras op massatransport nabij het grensvlak numeriek onderzocht. Een constante stofconcentratie werd opgelegd op de gas-vloeistofgrensvlakken, terwijl de vaste wand tussen de gasbellen in als impermeabel werd verondersteld. Als zodanig vormde dit model een tweedimensionale voorstelling van een microporeus membraan. Ondanks het feit dat massa alleen uitgewisseld werd aan de gas-vloeistofgrensvlakken van de bellenmatras, voorspellen deze resultaten een aanzienlijke toename in massatransport ten opzichte van niet-glibberige oppervlakken met een uniforme stofconcentratie. De vermeerdering hangt sterk af van de protrusiehoek van de bellen, de bedekking van het oppervlak met bellen en de stromingscondities in het kanaal. Daarnaast verdwijnt de versterking van het transport wanneer de gasbellen zeer klein zijn ten opzichte van de karakteristieke grootte van het kanaal. Aangezien de mate van slip schaalt met de grootte van de bellen, wordt het transport nabij kleine gasbelletjes gedomineerd door diffusie. Als 
gevolg daarvan kan de poreuze en glibberige bellenmatras voor kleine gasbelletjes beschouwd worden als zijnde volledig verzadigd met stof en als niet-glibberig.

Om de verhouding tussen hydrodynamische slip en grensvlaktransport op een meer globaal niveau te onderzoeken, hebben wij het klassieke Graetz-Nusseltprobleem uitgebreid naar continuümstroming met homogene wandslip. Dit probleem betreft warmte- of massatransport tussen een hydrodynamisch ontwikkelde laminaire stroming en een cylindrische buis met een constante wandtemperatuur of -concentratie. Zoals beschreven in hoofdstuk $\mathbf{6}$ hebben wij een analytische uitdrukking voor het Nusselt- of Sherwoodgetal in het ontwikkelende gebied afgeleid die geldig is voor elke waarde van de sliplengte. Het omvat ook de klassieke oplossingen voor slipvrije Hagen-Poiseuillestroming en voor afschuifspanningsvrije propstroom. De Nusselt- en Sherwoodgetallen voor gedeeltelijke slip gaan langzaam over van de onderste naar de bovenste klassieke limiet wanneer de sliplengte wordt vergroot van nul tot oneindig. Een fysische interpretatie van de resultaten laat zien dat het mechanisme voor warmte- dan wel stofoverdracht in het ontwikkelende gebied afhangt van het snelheidsprofiel in de thermische of massagrenslaag. Het relatieve belang van de slipsnelheid bepaalt of het mechanisme overeenkomt met het transportmechanisme voor slipvrije stroming of voor afschuifspanningsvrije stroming. Het transport in het ontwikkelde gebied, waar de thermische of massagrenslaag groter is dan de buisradius en het Nusselt of Sherwoodgetal constant is, hangt alleen af van het snelheidsprofiel in de buis.

Slipstroming over superhydrofobe oppervlakken is zeer heterogeneen, aangezien deze oppervlakken worden gekarakteriseerd door een patroon van (idealiter) slipvrije and afschuifspanningsvrije domeinen. Hoofdstuk 7 betreft de uitbreiding van het Graetz-Nusseltprobleem naar warmte- en massatransport over heterogeen glibberige, superhydrofobe oppervlakken. De randvoorwaarden zijn verschillend voor warmte- en stofoverdracht. Wanneer warmteoverdracht wordt beschreven heeft de niet-glibberige vaste wand een constante wandtemperatuur en kan, door het verschil in thermische conductiviteit, het glibberige gas-vloeistofgrensvlak als adiabatisch beschouwd worden. Voor massatransport heeft, daarbij transport nabij een gasgevuld membraan in ogenschouw nemend, het gas-vloeistofgrensvlak een constante wandconcentratie en is de vaste wand impermeabel. Aangezien alleen voor stofoverdracht de locaties voor hydrodynamische slip en massa-uitwisseling samenvallen, is massa-overdracht sneller dan thermisch transport. Over het algemeen is thermische diffusie sneller dan massadiffusie. Derhalve kan axiale conductie niet worden genegeerd wanneer warmteoverdracht in microscopische systemen wordt beschreven. Voor massatransport heeft axiale diffusie slechts een beperkte invloed op de overdrachtssnelheid.

In hoofdstuk 8 beschrijven wij de ontwikkeling van een uit polydimethylsiloxaan gemaakt microfluïdisch platform voor elektrodialyse dat een stapel van alternerend positief en negatief geladen hydrogelen bevat. De wand is heterogeen 
en bevat domeinen die impermeabel zijn. Onder een elektrisch potentiaalverschil worden ionen selectief door de anion- en kationuitwisselingshydrogelen getransporteerd. Dit leidt tot ofwel verrijking ofwel uitputting van de instromende zoutoplossingen. Voor voldoende hoge voltages zagen wij de vorming van vortices, welke convectief transport nabij de hydrogelgrensvlakken in het overlimiterende stroomgebied kunnen versterken. De chip maakt het mogelijk om ionentransport in de hydrogelen te visualiseren, wat vervolgens gekoppeld kan worden aan het transport nabij de grensvlakken en in de kanalen. Dit experimentele elektrodialyseplatform kan zo bijdragen aan een beter begrip van de verschillende transportverschijnselen die gerelateerd zijn aan elektrodialyse.

In hoofdstuk 9 worden de voornaamste conclusies gegeven van het in deze dissertatie beschreven werk, gevolgd door enkele suggesties voor toekomstig onderzoek naar glibberige oppervlakken en het gebruik daarvan voor het versterken van grensvlaktransport. Deze betreffen de toepassing van superhydrofobe oppervlakken in grootschalige processen die microscopische structuren bevatten, de versterking van oppervlaktegedreven stromingen middels hydrodynamische wandslip en de invloed van visco-elastische vloeistoffen op slip en de mogelijke toepassing daarvan. 




\title{
CHAPTER 1
}

\section{Transport near slippery interfaces: an introduction}

\begin{abstract}
This introductory chapter starts with a historical overview of the long debate the scientific community had regarding the correct boundary condition to apply at the interface between a flowing fluid and a solid wall: slip or no-slip? Based on experimental data, the community became slowly convinced that a fluid adjacent to a solid wall had no relative velocity to that wall. This is expressed by the no-slip boundary condition. However, the arrival of micro- and nanoscale technologies soon led to a revived discussion on the correct boundary condition. Nowadays, it is believed that smooth hydrophobic surfaces can exhibit wall slip: experimental observations indicate that a fluid can have a finite velocity relative to the wall. For such surfaces, slippage is limited though. Superhydrophobic surfaces are characterised by much more wall slip, which arises from the combination of microscale surface roughness and hydrophobicity. The gas present in the micro-structures of a superhydrophobic surface makes it slippery. Consequently, superhydrophobic surfaces can significantly reduce hydrodynamic friction between a flowing liquid and the wall when compared to flat, non-slippery surfaces. As discussed in this chapter, the amount of wall slip depends on many parameters, such as the surface topology, the flow direction and the composition and curvature of the interface. Because of the drag-reducing properties of superhydrophobic surfaces, they can also be used for increasing transport of heat and mass near the interface. Wall slip leads to fluid flow and consequently increased convection near the wall, and therefore can enhance interfacial transport. For both drag reduction and enhancement of heat/mass transfer, it is required that the surface heterogeneity has a scale that is similar to the characteristic length of the system.
\end{abstract}




\subsection{Slip or no-slip?}

\subsubsection{A historical overview}

Which conditions need to be satisfied by a moving fluid that is in contact with a solid body? This question had occupied the scientific community for a long time. Experiments by scientists like Bernouilli, Du Buat and Coulomb all suggested that the fluid layer adjacent to the surface had no relative velocity to it $[1,2]$, as illustrated in Fig. 1.1(a). These observations were in contradiction with the equations describing fluid flow as derived by Euler, in which the concept of friction was neglected. To bridge the gap between mathematical theory and engineering practice, Navier published in 1823 a new set of hydrodynamic equations that described viscous flow [3]. He derived those based on a modification of Euler's equations of potential flow by taking into account the forces acting between the molecules in the fluid. In the case of a flowing fluid with velocity $\mathbf{u}$, he assumed that 'the repulsive actions of the molecules are increased or diminished by a quantity that is proportional to the velocity with which the distance between the molecules decreases or increases' $[2,3]$. This resulted in an additional term to Euler's equations, $\mu \nabla^{2} \mathbf{u}$, although Navier did not recognise that this multiplicative constant $\mu$ denoted the viscosity of the fluid [4]. The new governing equation for flow with friction then read

$$
\rho\left[\frac{\partial \mathbf{u}}{\partial t}+(\mathbf{u} \cdot \nabla \mathbf{u})\right]=-\nabla p+\mu \nabla^{2} \mathbf{u}+\mathbf{f},
$$

which is nowadays known as the Navier-Stokes equation for an incompressible Newtonian fluid. Here, $\rho$ is the density of the fluid, $p$ is the pressure and $\mathbf{f}$ is a body force. Besides that, incompressibility of the fluid implies that the density is constant, resulting in the following simplified continuity equation:

$$
\nabla \cdot \mathbf{u}=0
$$

Stokes independently arrived at the same equation in 1845, although the NavierStokes equation was also rederived or rediscovered by Cauchy, Poisson and Saint-Venant $[2,4]$.

Having derived an hydrodynamic equation that accounted for fluid resistance and flow retardation, Navier applied his theory to the experimental results of Girard, who measured the outflow of water from copper capillary tubes. As Girard did, Navier initially assumed the walls were covered with a very thin layer of immobilised fluid $[1,5]$ :

Il existe contre les parois solides une chouche extrêmement mince de fluide immobile, ou que ces parois sont formées de la substance même du fluide, qui aurait été solidifiée. Alors la vitesse u devra être 
nulle dans toute l'étendue de ces parois.

The fluid next to this layer of stagnant fluid would then have a uniform velocity and thus move as a plug through the tubes. Using the assumption of a vanishing velocity $\mathbf{u}$ at the wall, Navier found that he could only partially explain the results of Girard. In addition, the no-slip boundary condition could not clarify why Girard observed a difference in discharge for different liquids (water and mercury) or for different tube wall materials (glass and copper). For that reason, Navier gave up the no-slip boundary condition and proposed a new boundary condition [3]. Using the same molecular hypotheses on which his equations of motions were based, 'he deduced that there is slipping at the solid boundary, and this slipping is resisted by a force proportional to the relative velocity' [1]. This new condition read

$$
E \mathbf{u}+\mu \partial_{\perp} \mathbf{u}_{\|}=\mathbf{0}
$$

Here, $E$ is a molecular constant or a surface coefficient that accounts for the attraction/repulsion between the molecules of the liquid and the wall [6]. As Navier wrote:

La constante $\mu$ représente (...) la résistance provenant du glissement de deux couches quelconques l'une sur l'autre. (...) La constante E représente (...) la résistance provenant du glissement de cette couche sur la paroi.

La lettre E représante une constante dont la valeur sera donnée par l'expérience, d'après la nature de la paroi et du fluide, et qui peut être regardée comme la mesure de leur action réciproque.

$\partial_{\perp}$ is the derivative normal to the surface, and $\mathbf{u}_{\|}$is the velocity parallel to the surface. It follows that the fraction $\mu / E$ corresponds to a length that is zero when there is no slip. In the case of a free surface, $E=0$. Using this boundary condition, which is illustrated in Fig. 1.1(b), Navier was able to explain Girard's results.

Nevertheless, it took a while before consensus was reached on the condition that correctly described the interface between a fluid and a solid wall [1]. Stokes was inclined to assume a no-slip boundary condition, but since his predictions did not agree with the experiments then known he hesitated between the no-slip boundary condition and Navier's slip condition. He finally thought that the assumption of no-slip was most plausible. Girard's experiments were repeated with much greater accuracy by Poiseuille and Hagen, and their results suggested that the liquid at the tube walls must have a negligible velocity. Or, in the case there would be a stagnant liquid layer at the wall, Hagen found that the thickness of this layer had to be very thin [7]: 
Ich erwähne noch, dass die angenommene Dicke der ruhenden Wasserschicht (...) nicht stärker ist, als das allerfeinste Brief-Papier.

Hagen, in accordance with Poiseuille, also reported that the fluid velocity increases from zero at the walls to a maximum in the middle of the tube [8]. As such, he considered the common view that a fluid would flow as a plug through a tube as incorrect.

Diese gewönliche Annahme, dass das Wasser sich in allen Theilen des Querschnittes der Röhre mit gleicher Geschwindigkeit bewegt, führt also zu keinem passenden Resultate. (...) So scheint die Voraussetzung zulässig, dass die ganze Wassermenge sich in concentrische hohle Röhren von sehr geringer Dicke zerlegt, und von diesen sich eine in der anderen fortschiebt, und zwar eilen sie gleichmässig einander vor, nachdem jede schon beim Eintritt in die Durchflussröhre die ihr zukommende Geschwindigkeit angenommen hat. Indem aber die äusserste Wasserröhre an der Wand der Durchflussröhre haftet, so ist die Geschwindigkeit derselben gleich Null, und es tritt sonach in jeder Secunde nicht ein Wassercylinder aus der Röhre, sondern ein Wasserkegel, der den Querschnitt der Röhre zur Grundfläche und die Geschwindigkeit des mittleren Fadens zur Höhe hat.

Maxwell arrived at the same boundary condition as Navier did. Maxwell derived that in the case of a rarified gas flow at constant temperature, the gas is slipping over the surface as described by Navier's condition: $u=G \partial_{n} u[9]$.

If, therefore, the gas at a finite distance from the surface is moving parallel to the surface, the gas in contact with the surface will be sliding over it with the finite velocity $v$, and the motion of the gas will be very nearly the same as if the stratum of depth $G$ had been removed from the solid and filled with the gas, there being now no slipping between the new surface of the solid and the gas in contact with it.

The coefficient $G$ was introduced by Helmholtz and Piotrowski under the name of Gleitungs-coefficient, or coefficient of slipping. The dimensions of $G$ are those of a line.

Maxwell also found that the slipping coefficient $G$ is proportional to the mean free path of a molecule, and that 'hence at ordinary pressures $G$ is insensible.' This implies that at ambient pressure, the wall can be considered as non-slippery.

The scientific community thus slowly became convinced that, despite the lack of any conclusive proof, a fluid adjacent to a solid wall could be treated as having no relative velocity to the wall $[1,10,11]$. If there would be any slip between a fluid and a wall, the amount of slip would be too small to significantly affect 
(a) No-slip



(b) Wall slip

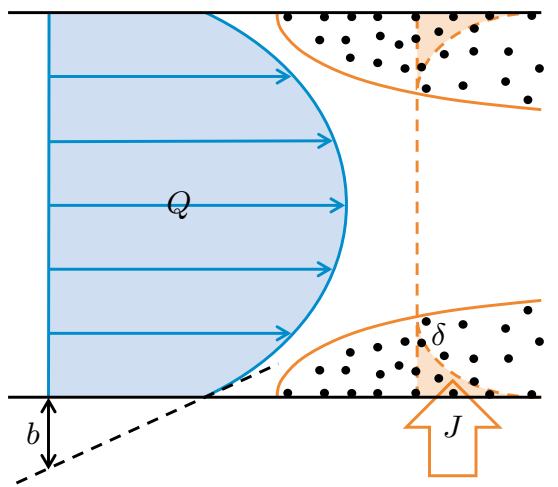

Figure 1.1 - For a long time, the scientific community was convinced that a fluid adjacent to a wall had no relative velocity to that wall. (a) This is expressed by the noslip boundary condition, which in the case of pressure-driven flow results in a parabolic velocity profile. (b) However, recent studies have shown that some surfaces are slippery, which is quantified by a slip length $b$. For a given driving force, this increases the flow rate $Q$. Wall slip also results in advection near the interface between the wall and the fluid. This can enhance interfacial transport, yielding a higher flux $J$ and a reduced boundary layer thickness $\delta$.

theoretical deductions. This conviction, which was an acknowledged truth until the end of the 20th century, was based on the agreement between experimental observations and theoretical analyses assuming the no-slip boundary condition.

\subsubsection{The slip boundary condition}

In the case of a slippery wall, the boundary condition is defined as follows [11]:

$$
\mathbf{u}_{\|}=b \mathbf{n} \cdot\left[\nabla \mathbf{u}+(\nabla \mathbf{u})^{\mathrm{T}}\right] \cdot(1-\mathbf{n} \mathbf{n}) .
$$

Often, this boundary condition is rewritten into two complementary equations, which are the impermeability condition and Navier's slip condition. For a twodimensional flow with the $y$-axis normal to the surface, this yields the following:

$$
\begin{gathered}
\mathbf{u} \cdot \mathbf{n}=0, \\
u_{b}=-\left.b \frac{\partial u}{\partial y}\right|_{\text {wall }} .
\end{gathered}
$$

The first equation says that the velocity component normal to the surface is zero: the wall is impermeable. The second equation states that the slip velocity $u_{b}$ parallel to the surface is proportional to the slip length $b$ and the shear rate $\partial_{y} u$ 
at the wall. The slip length $b$ is then equivalent to, see Eq. (1.3), the ratio $\mu / E$ of dynamic viscosity over friction coefficient. As illustrated in Fig. 1.1(b), it can be considered as the hypothetical distance below the surface where zero fluid velocity would be obtained when linearly extrapolating the velocity profile at the wall.

\subsection{Momentum transport}

\subsubsection{Renewed interest in the no-slip boundary condition}

When would the effect of wall slip on the flow rate be significant? Or, as also illustrated in Fig. 1.1, which slip length is required to reduce hydrodynamic drag or enhance the flow rate significantly? Assuming that at the interface between the wall of a cylindrical tube with radius $R$ and an incompressible fluid Navier's slip condition holds, and that the fluid flow is driven by a pressure gradient in the $x$-direction, we find the following expression for the flow rate $Q$ :

$$
Q=-\frac{\pi R^{4}}{8 \mu} \frac{\partial p}{\partial x}\left(1+\frac{4 b}{R}\right) .
$$

It immediately follows that wall slip is only significant when the ratio of slip length $b$ to tube radius $R$ is larger than $10^{-2}$. Thus, to increase the flow rate by ten percent for a tube with a radius of $1 \mathrm{~mm}$, the slip length would have to be $25 \mu \mathrm{m}$. As will be discussed later in more detail, slip lengths of this size have only been measured in slippery and structured superhydrophobic systems (see the reviews [12-18] and the references therein). However, superhydrophobic surfaces have only been used for drag reduction since 2000. Before that time, most studies concerned surfaces showing intrinsic or molecular wall slip, for which typical slip lengths are of the order of tens of nanometres (see the reviews $[10,11,14]$ and the references therein). This clarifies why for a long time most experiments empirically confirmed the validity of the no-slip boundary condition: intrinsic slip lengths are too small to be observed in large-scale or macroscopic systems.

However, at the end of the 20th century it became technologically possible to manufacture microscale and later also nanoscale devices. This led to the emergence of new research fields in which microfluidic and nanofluidic devices

play an important role. The ability to manufacture such devices makes it possible to investigate physical phenomena that act on small scales, like interfacial wall slip, with high precision and accuracy [10, 19].

\subsubsection{Slippery smooth surfaces}

The possibility to engineer microscale or smaller systems led to a revival of studies investigating the phenomenon of wall slip, as the generally accepted no-slip boundary condition was questioned again in some early studies [20, 21]. The 


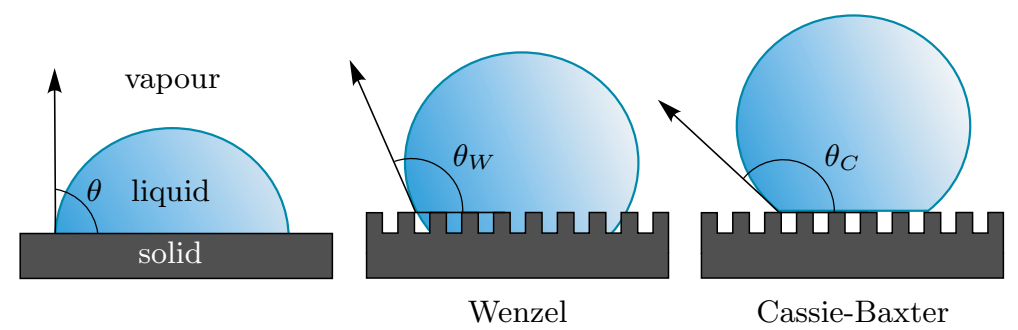

Figure 1.2 - Wetting of a solid surface by a liquid depends on the adhesive forces between the solid and the liquid, the cohesive forces within the liquid, but also on the texture of the surface. In the case of a flat, homogeneous surface, the contact angle $\theta$ is given by Young's law as defined in Eq. (1.8). The contact angle usually is larger in the case of rough or textured surfaces, for which two wetting states can be distinguished. In the Wenzel state the liquid penetrates into the surface structures. In the Cassie-Baxter state, the space between the structures is filled with a gas and the liquid is resting on top of the hybrid gas-solid surface. The Cassie-Baxter contact angle $\theta_{C}$ is defined in Eq. (1.11). Figure adapted from [25].

first studies investigated the possibility of wall slip by varying the hydrophobicity of a wall, indicating that the slip length increases with decreasing wettability $[10,11,14]$. The wettability is characterised by the contact angle $\theta$, which is the angle at which the gas-liquid interface of a droplet meets the liquid-solid interface. This is depicted in Fig. 1.2. In the case of a partially wetting surface (where the spreading parameter $S=\sigma_{g s}-\sigma_{g l}-\sigma_{l s}<1$ ), the contact angle $\theta$ is defined by Young's law,

$$
\sigma_{g s}=\sigma_{l s}-\sigma_{g l} \cos \theta,
$$

which relates the interfacial tension $\sigma$ between the gas $(g)$, liquid $(l)$ and solid $(s)$ phases. Since the interfacial tension in fact is the free energy per unit area, the contact angle is therefore fixed by the chemical nature of the different phases [22-24]. As follows from the Young-Dupré equation,

$$
W_{l s}^{a}=\sigma_{g l}(1+\cos \theta)
$$

the work of adhesion $W_{l s}^{a}$ between the liquid and solid phase decreases when the contact angle or surface hydrophobicity increases.

Although it remained a discussion for quite some time and it still is difficult to prove $[26,27]$, it is now believed that only hydrophobic, smooth surfaces display wall slip, whereas the amount of slip is negligible for highly wettable surfaces $[10,28,29]$. For smooth hydrophobic surfaces, reported slip lengths are typically in the range of $1 \mathrm{~nm}$ to $100 \mathrm{~nm}[10,11,14]$. This concerns intrinsic or molecular slip, which is illustrated in Fig. 1.3 $(a)$. This type of slip is actual or 'true' slip: fluid molecules sliding over the solid molecules of the wall. 
Even smooth surfaces, however, are rough on an atomic scale. The magnitude and length scale of the surface roughness appeared to be an important factor regarding the slipperiness of a wall. However, the results are ambiguous: when increasing the surface roughness, some studies reported an increase in slip, while other demonstrated a decrease in slip [10]. There are some explanations why roughness could reduce friction. In the case of a wetted surface, liquid molecules can be trapped in the pits on the surface. The liquid then slides over a heterogeneous layer of solid and immobilised (and possibly spatially ordered) liquid molecules, thereby reducing the friction at the wall [10]. However, this raises the question where the actual boundary should be located, as a shift of the reference plane (used to calculate the slip length) below the peaks of the wall roughness frequently leads to a recovery of the no-slip boundary condition.

Another possible issue that can affect the measurement of intrinsic slip lengths is the presence of nanobubbles or gas-covered regions at the rough or hydrophobic surface [10, 28, 30, 31]. These nanobubbles or gas layers are formed when the gas entrained by the liquid flow nucleates on the hydrophobic and/or rough surface. This affects the amount of slip measured, as the gas bubbles reduce the amount of friction considerably. An example is the study of Tretheway and Meinhart [32], who measured a slip length of $1 \mu \mathrm{m}$ in a channel of $30 \times 300 \mu \mathrm{m}$ with smooth, hydrophobised walls using micro-particle image velocimetry. Later, however, they found that this appeared to be an apparent slip length due to the formation of a lubricating gas layer on the channel walls [33].

In the case of a gas-lubricated liquid-solid interface, assuming continuity of stress, the slip length $b$ is of the order of $[16,34]$

$$
b=\delta\left(\frac{\mu_{l}}{\mu_{g}}-1\right) \simeq \delta \frac{\mu_{l}}{\mu_{g}} .
$$

In the case of nanobubbles, the thickness $\delta$ of the gas layer is typically $20 \mathrm{~nm}$. For air-water systems, the viscosity ratio is $\mu_{l} / \mu_{g} \sim 50$ and we find a slip length of $b \sim 1 \mu \mathrm{m}$. This means that for a surface covered with nanobubbles or with a gas layer, the measured slip length must be something else than the intrinsic slip length $b_{i}$.

As illustrated in Fig. 1.3(b), in case of a gas layer covering the complete surface, an apparent slip length $b_{a}$ is measured. Because of the lubricating gas layer, it appears that the surface is slippery, while actually the no-slip boundary condition holds at the solid wall. When the surface is only partly covered with nanobubbles, as depicted in Fig. 1.3(c), slippage is characterised by an effective slip length. This effective slip length $b_{f}$ is an 'average' value for the heterogeneous surface, which is characterised by regions of high slip $(b \gg 0)$ and zero slip $(b=0)$. The effective slip length therefore represents the amount of slip that is experienced by the flow on scales larger than the surface heterogeneities. 


\subsubsection{What causes wall slip?}

What is the actual physical origin of wall slip? As already hinted in the previous section, there are two phenomena that are of importance: the intermolecular forces or attraction between the molecules of the fluid and the solid, and the roughness of the surface.

Intermolecular forces inhibit slipping of the fluid over the solid surface. That is, fluid molecules do not move relative to the wall, because they adhere to the surface. The friction arising from these intermolecular forces depends on the chemical properties of both fluid and wall. In 1860, Helmholtz already wrote [35]:

So bestätigt sich durch die hier vorliegenden Versuche, dass die chemische Beschaffenheit der Wand auf die Bewegung der Flüssigkeiten nicht in allen Fällen einflusslos ist.

Sticking of molecules to the wall does not imply that near the wall molecules are fully immobilised. It remains a dynamical system, since by Brownian motion individual molecules stick to and come off the wall. As such, the slip as well as the no-slip boundary condition do not correspond to the actual statistical-mechanical conditions prevailing at the solid wall [36], but are only a continuum description without any microscopic information $[19,37]$. But, when the attractive forces between a fluid and a wall become weaker, the wettability of a surface decreases and, as a consequence, also the friction at the wall decreases. Note that there also exists an hypothesis saying that, in the case of a weakly attractive solid, a depletion layer is formed near the wall, which again would suggest that the no-slip boundary condition holds on smooth non-wetting surfaces and that often an apparent slip length is measured [38].

However, wall slip is inhibited by roughness. Since essentially all surfaces are rough at the microscale, the energy that is dissipated when a fluid flows over these irregularities brings it to rest. It does not matter how strong the intermolecular forces between the fluid and solid molecules are. Richardson demonstrated that, for rough surfaces that are subject to either a no-slip or no-shear condition at their walls, the roughness by itself ensures that on a macroscopic scale no-slip is the perceived condition at the wall [39]. However, this view also implies that for a perfectly smooth surface, wall slip is infinite and no stress is experienced by the fluid at the wall [19].

In reality, it is the combination of both phenomena that determines the slipperiness of a surface. Roughness does increase the flow resistance, but can also lead to dewetting of the surface. However, even surfaces that are very smooth and non-wetting, are not shear-free. A simple force balance shows that a critical shear force needs to be overcome. The attractive force of $F \sim \sigma a$, where $\sigma$ is the interfacial energy (or surface tension) and $a$ is the size of the molecule, has to equal the hydrodynamic stress $F \sim \mu a^{2} \gamma$, where $\gamma$ is the shear rate of the fluid 
flow. For that reason, a critical shear rate of $\gamma \sim \sigma /(\mu a)$ needs to be met before the molecule can actually slip over the surface [40]. This theoretical argument could explain why for Newtonian fluids often a dependency of wall slip on the shear rate is reported [10].

\subsubsection{Superhydrophobicity}

Since intrinsic slip lengths are too small to significantly reduce friction in systems larger than the microscale, it was suggested that the actual liquid-solid boundary should be replaced by a gas-liquid boundary by for instance covering the surface with bubbles [12]. The strongly water-repellent leaves of the lotus and other plants, and the legs of various insects and spiders possess such properties [41-43]. The combination of surface roughness and wall hydrophobicity makes water droplets to sit on top of the wall roughness, thereby promoting the formation of air pockets in between the liquid and the surface structures (see Fig. 1.3(c)). Large contact angles and low contact angle hysteresis, although the precise definitions are still subject of research [44-47], characterise such surfaces as superhydrophobic. Bico et al. showed that the fraction of the solid that contacts the surface, and not the surface roughness itself, determines the (super)hydrophobicity of the surface [48]. Air trapping can be enhanced by utilising structures with sharp edges, promoting pinning of the gas-liquid interface at these edges [24, 49]. Experiments showed that the flow resistance of water droplets sliding and/or rolling down inclined surfaces was reduced greatly when the surface was superhydrophobic [50, 51].

Superhydrophobic surfaces are characterised by very large contact angles, which can reach values of almost $180^{\circ}$. Engineered superhydrophobic surfaces, illustrated in Fig. 1.3 $(d)$, usually possess a well-defined roughness at scales larger than rough surfaces [17]. Two different wetting states can be distinguished. In the Wenzel state, the liquid (droplet) impregnates the surface roughness, which is expected to decrease wall slip [12]. In the Cassie-Baxter state, the liquid (droplet) is lying on top of the surface roughness. The hydrophobicity of the wall prevents the liquid from filling the structures, which then, consequently, are filled with a gas. The contact area between liquid and solid is therefore very small, thereby reducing friction. These two states are illustrated in Fig. 1.2. The contact angle $\theta_{C}$ of the Cassie-Baxter state, given by [52]

$$
\cos \theta_{C}=\phi_{s}(1+\cos \theta)-1
$$

depends on the solid-liquid fraction $\phi_{s}$ of the hybrid surface and the contact angle $\theta$ of liquid on the flat solid itself. A superhydrophobic surface is also characterised by a low contact angle hysteresis, which is, as the Cassie-Baxter contact angle, a function of the shape, size and density of the surface structures [53].

In the case a surface is in the Cassie-Baxter state, it necessarily follows that 
(a) Intrinsic wall slip

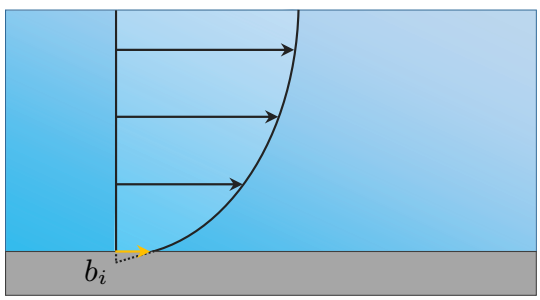

(c) Effective wall slip

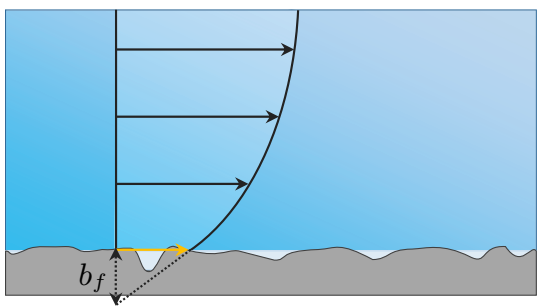

(b) Apparent wall slip

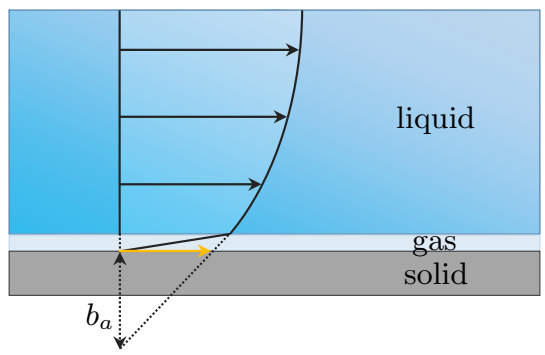

(d) Effective wall slip

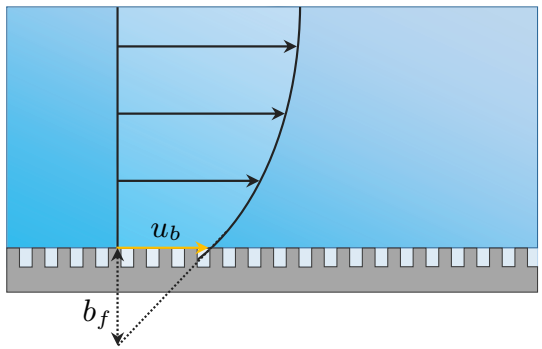

Figure 1.3 - Different types of wall slip can be distinguished. In the case of $(a)$ intrinsic wall slip, fluid molecules slide over the solid molecules of the (usually hydrophobic) wall with a slip velocity $u_{b}$. Intrinsic slip lengths $b_{i}$ are typically of the order of tens of nanometres. In the case of $(b)$ apparent wall slip, there is a lubricating layer of a low viscosity fluid between the outer flow and the wall. It appears therefore that the wall is slippery, while actually the no-slip boundary condition holds. The apparent slip length $b_{a}$ scales with the viscosity ratio of both fluids. Effective wall slip is encountered for naturally $(c)$ or engineered $(d)$ rough superhydrophobic surfaces. The effective slip length $b_{f}$ scales with the length scale $L$ of the surface structures, such that typically $b_{f} / L=\mathcal{O}(1)$. However, the exact value depends on the solid fraction of the surface, the orientation of the structures with respect to the flow and the interface curvature.

there exists a limit to the Laplace pressure before it transitions to the Wenzel state $[14,54]$. This pressure difference equals to

$$
\Delta p=\sigma R_{c} \cos \theta
$$

where $R_{c}=\left(R_{1}^{-1}+R_{2}^{-1}\right)$ and $R_{1}$ and $R_{2}$ are the principal radii of curvature. The maximum pressure difference that the gas-liquid interface can withstand is therefore inversely proportional to the spacing between the structure elements of the surface. For a given application or for given operating conditions, this provides an upper limit to the spacing of the surface structures, and therefore also to the maximum amount of slip that can be obtained for a superhydrophobic surface. 


\subsubsection{Slippery superhydrophobic surfaces}

For these reasons, the use of superhydrophobic surfaces was identified as a promising route to reduce flow friction in small channels. Typical surface structures are pillars, grooves and cavities, as shown in Fig. 1.4. Watanabe et al. [55] reported in 1999 a $14 \%$ drag reduction for laminar flow through tubes with a diameter of $10^{1} \mathrm{~mm}$ by using pressure drop measurements. This corresponds to a slip length of $\mathcal{O}\left(10^{2} \mu \mathrm{m}\right)$. They attributed this to the presence of a gas in the structures of the rough, water-repellent wall. With that, and probably unintentionally, they were among the first to quantify slippage over superhydrophobic surfaces.

By using molecular dynamics simulations, Cottin-Bizonne et al. [56, 57] confirmed that superhydrophobic surfaces could be used to reduce drag. Experimental studies followed, using various techniques to quantify the effect of wall superhydrophobicity on the flow resistance. Using pressure drop measurements, and later extended by using micro-particle image velocimetry ( $\mu \mathrm{PIV})$, Ou et al. $[58,59]$ measured slip lengths up to $20 \mu \mathrm{m}$ for silanised superhydrophobic silicon surfaces with micrometre-sized posts and ridges. The results were validated by numerical simulations. They reported that the slip length increases with decreasing solid fraction $\phi_{s}$, with ridges positioned parallel to the flow direction outperforming regular arrays of posts.

Choi and Kim [60-62] measured effective slip lengths through torque measurements using a rheometer on silicon surfaces containing needle-like structures, and reported slip lengths of $20 \mu \mathrm{m}$ for water. Joseph et al. [63], however, reported much smaller slip lengths of a few micrometres for flow over a superhydrophobic surface containing carbon nanotubes. They used $\mu \mathrm{PIV}$ to quantify the slip flow near the surface at submicrometre resolution.

Truesdell et al. [64] reported, using $\mu \mathrm{PIV}$ and a Couette cell, slip lengths of tens of micrometres for flow over longitudinal grooves with a spacing of $25 \mu \mathrm{m}$. Based on simulations, they concluded that these large slip lengths originate from other mechanisms; possibly from the formation of a vapour layer between the fluid and the surface. Maynes et al. [65, 66] combined numerical and analytical work with experiments (measuring flow rate and pressure drop), considering both flow over transverse and longitudinal grooves. They reported that, due to vapour flow inside the gas-filled cavities of the micro-structured surfaces, the assumption of zero-shear at the gas-liquid interface leads to a certain overestimation of the drag reduction. For cavity depths similar to or larger than the hydraulic channel diameter, applying a no-shear condition at the gas-liquid interface is a good approximation though. Besides for longitudinal grooves, the slip length decreases with increasing Reynolds number when $R e>10^{2}$ [65, 67].

Hydrophobic, nano-structured surfaces were fabricated by Choi et al. [68], who inferred a slip length of $140 \mathrm{~nm}$ for longitudinal and of $60 \mathrm{~nm}$ for transverse grooves from flow rate and pressure drop measurements. They used channels 
of approximately $5 \mu \mathrm{m}$ high and a surface consisting of ridges with a $230 \mathrm{~nm}$ pitch, having a solid fraction of $\phi_{s}=0.3$. Lee et al. [69], on the other hand, fabricated surfaces with posts and longitudinal grooves that were characterised by very large pitch sizes up to $200 \mu \mathrm{m}$ and gas fractions approaching unity. By using a rheometer, they found effective slip lengths approaching $200 \mu \mathrm{m}$.

Byun et al. [70] used $\mu \mathrm{PIV}$ to measure the slip length in superhydrophobic polydimethylsiloxane (PDMS) channels. They reported slip lengths up to $5 \mu \mathrm{m}$ for channels with transverse grooves of $21 \mu \mathrm{m}$ width and with a solid fraction of $\phi_{s}=0.4$. Tsai et al. [71] utilised $\mu \mathrm{PIV}$ to quantify the effective slip length for PDMS surfaces containing longitudinal grooves (see Fig. 1.4(b)) with a width between 8 to $32 \mu \mathrm{m}$ and a gas fraction of 0.5 . They determined the slip lengths to be $3 \mu \mathrm{m}$, and found the value to depend on the shape of the gas-liquid menisci.

Recently, Schäffel et al. demonstrated that the local slip length can be anisotropic and thus can vary with position [72]. They used a surface containing a square pattern of $12 \mu \mathrm{m}$ diameter pillars with a pitch of $20 \mu \mathrm{m}$. The effective slip length amounted to $2 \mu \mathrm{m}$.

\subsubsection{Analytical relationships}

All of these experimental studies on slip over superhydrophobic surfaces demonstrate that the amount of wall slip not only depends on the type of surface patterning, which are typically posts, grooves and cavities, but also on the orientation of the structures with respect to the flow. Philip [75, 76] and later also Lauga and Stone [77] derived that in the case of a surface that consists of an alternating pattern of no-slip and no-shear stripes or grooves, the effective slip length $b_{f}$ is given by

$$
b_{f}=-\alpha \frac{L}{\pi} \ln \left[\cos \left(\varepsilon \frac{\pi}{2}\right)\right],
$$

where $L$ is the period length between each no-slip and no-shear unit, and $\varepsilon=$ $\left(1-\phi_{s}\right)$ is the surface porosity or the surface fraction that is perfectly slippery. The prefactor $\alpha$ depends on the orientation of the flow with respect to the grooves. In case of parallel flow $\alpha=1$, whereas for perpendicular flow $\alpha=1 / 2$. These two situations correspond to the maximum and minimum amount of wall slip that can be obtained for flow over grooved superhydrophobic surfaces [78-80].

Comparison of Eq. (1.13) with the experimentally determined slip lengths discussed in Sec. 1.2.5, which vary from tens of nanometres to approximately hundred micrometres, reveals that the non-dimensionalised slip length $b_{f} / L$ is often of the order of one, i.e. $b_{f} / L=\mathcal{O}(1)$.

Ybert et al. [81, 82] showed that for a surface with a vanishing solid fraction of $\phi_{s} \rightarrow 0$, which is in terms of slippage most interesting, the shear stress $\tau \sim \phi_{s} \mu \gamma_{s}$. The shear rate $\gamma_{s}$ at the solid surface is determined by the slip velocity $u_{b}$ around the solid fraction of the surface, which is characterised by the length scale $a$. Thus, 

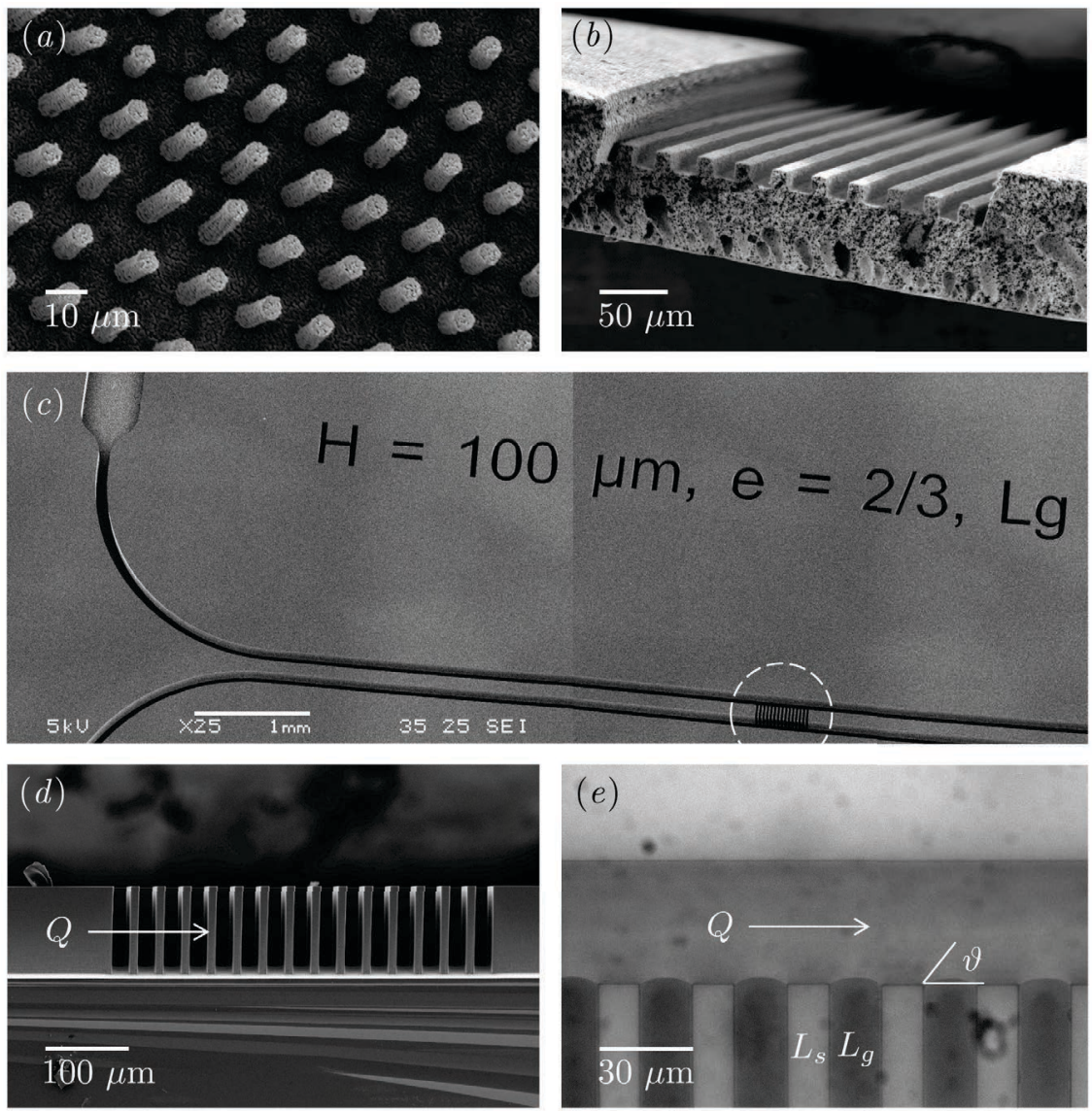

Figure 1.4 - Engineered superhydrophobic surfaces often contain surface structures that include pillars, grooves and cavities. When in the Cassie-Baxter state, these surfaces exhibit wall slip, thereby reducing hydrodynamic drag. This can enhance mass transport, as in for example oxygen transfer across the pillared porous membrane shown in $(a)$ [73]. The drag reducing properties also depend on the orientation of the micro-structures with respect to the flow. Longitudinal grooves, i.e. grooves in the direction of the flow as in (b), maximise wall slip [71]. Superhydrophobic surfaces can consist of transverse ribs as in $(c)$, with the encircled part corresponding to the image in $(d)$. For these surfaces, often referred to as bubble mattresses (e) [74], wall slip is a function of the protrusion angle $\vartheta$ and is maximum for an angle of approximately $10^{\circ}$.

$\gamma_{s} \sim u_{b} / a$. From Navier's slip condition, we find that $\tau \sim \mu u_{b} / b$. Substitution yields that for a surface with a vanishing solid fraction $\left(\phi_{s} \rightarrow 0\right)$

$$
b_{f} \sim \alpha \frac{a}{\phi_{s}}
$$

with $\alpha$ again being a prefactor that depends on the geometry of the structured 
surface. In the case of a surface with grooves, $\phi_{s}=a / L$ and thus $b_{f} \sim L$. This is in agreement with Eq. (1.13), as for $\phi_{s} \rightarrow 0$ or $\varepsilon \rightarrow 1$ we find that $b_{f} \sim-L / \pi \ln \phi_{s}$. Since as a practical limit $\phi_{s}<0.01$, this indeed reduces to $b_{f} \sim L$. For a surface with pillars, $\phi_{s}=(a / L)^{2}$ and therefore $b_{f} \sim L / \sqrt{\phi_{s}}$. This reveals that for surface geometries with stripes or grooves, effective slip lengths are of the order of the surface periodicity $L$. For pillared surfaces, with $b_{f} \sim L / \sqrt{\phi_{s}}$, slip lengths much larger than $L$ can be achieved. The amount of wall slip over surfaces with other geometries, such as fractal and checkerboard surfaces, have been subject of various analytical studies as well $[83,84]$.

Usually, the gas-liquid (often air-water) interfaces of a superhydrophobic surface are assumed to be shear-free or perfectly slippery. This condition is obtained when assuming that the viscosity ratio of liquid to gas is infinite, i.e. $\mu_{l} / \mu_{g} \rightarrow \infty$. Schönecker and Hardt $[85,86]$ investigated the influence of the flow patterns inside the surface cavities, with varying geometries, on the outer flow over these cavities. For viscosity ratios of $\mu_{l} / \mu_{g}>50$, cavities with a depth larger than their width and moderate surface coverages $\varepsilon$, their analytical and numerical results confirmed the accuracy of the assumption that the gas-liquid interface is shear-free. Under these conditions, Eq. (1.13) gives a reasonably accurate prediction of the effective slip length. By contrast, their study also explains why slip over liquid-liquid interfaces is hard to achieve. In the case of patterned surfaces, this is only possible when the flowing liquid has a much larger viscosity than the surface-infusing liquid.

The drag reducing properties of superhydrophobic surfaces are beyond questioning, but the degree of drag reduction depends (as with atomically rough surfaces, as discussed in Sec. 1.2.2) on the location of the reference plane [87, 88]. When locating this plane at the bottom of the surface structures, these structures reduce the effective cross-section of the channel. Still, for relatively shallow cavities and high viscosity ratios it is possible to achieve a considerable drag reduction.

\subsubsection{Curved gas-liquid interfaces}

For flat, hybrid gas-solid surfaces, the effective slip length is always positive. This may not be true for superhydrophobic surfaces containing curved gas-liquid interfaces: the amount of slip depends on the curvature of the bubble interface $[57,81,89]$, which is often characterised by the protrusion angle $\vartheta$. Steinberger et al. [90] found that the slip length decreases for increasing protrusion angles. Negative slip lengths were computed for positive protrusion angles exceeding a critical value, as confirmed in other studies [91]. This implies a superhydrophobic surface containing bubbles can increase the flow resistance. Teo and Khoo [92] showed numerically that with increasing protrusion angle, the wall shear stress near the contacts points of the wall and the bubbles decreases, while the static pressure difference between the front and rear halves of the bubble interfaces 
increases. This latter effect dominates beyond the critical protrusion angle, resulting in negative slip lengths.

Davis and Lauga [93] derived an analytical expression for the effective slip length for transverse shear flow over a bubble mattress in the dilute limit, i.e. for a surface coverage $\varepsilon=\left(1-\phi_{s}\right)<0.35$ [94], and showed that the slip length is a function of the protrusion angle:

$$
\frac{2 b}{L_{g}}=\pi \varepsilon f(\vartheta) .
$$

For protrusion angles larger than approximately $60^{\circ}$ wall slip becomes negative, denoting increased friction compared to a non-slippery surface. Later studies confirmed the general validity of this model, even for three-dimensional surfaces containing spherical bubbles [92, 94-96]. To prove this predicted dependency of wall slip on the protrusion angle experimentally, we fabricated silicon-based microfluidic devices that allowed active control of the bubble protrusion angle $\vartheta$ and we measured the effective slip length for $-5^{\circ}<\vartheta<45^{\circ}$ using $\mu \mathrm{PIV}$ [74] (chapter 2). This is also illustrated in Fig. 1.4(c-e). We demonstrated that, in agreement with numerical simulations, the slip length is maximum for (nearly) flat bubbles and subsequently decreasing with increasing protrusion angle.

The amount of slip in flow over a bubble mattress is maximum for a small, but positive optimum protrusion angle of $\vartheta \sim 10^{\circ}$ [92-94]. By considering the spatial distribution of viscous dissipation, we explained why wall slip is maximised when the bubble mattress is slightly bumpy [97] (chapter 3). Bubbles protruding in the channel act as obstacles and reduce the effective channel height, thereby increasing the dissipation in the bulk flow. At small scales, however, increasing the protrusion angle reduces the dissipation near the contact points of the no-slip channel wall and the no-shear bubble surfaces.

Hyväluoma and Harting [95] found that, considering infinitely long cylindrical bubbles, the slip length strongly depends on the flow direction. When the flow is parallel to the bubble slots, the slip length is always positive: the streamlines are straight and no roughness is encountered by the flow. The amount of slip still does depend on the protrusion angle though [94, 98-101].

\subsubsection{Shape, stability and contamination}

It is not only the protrusion angle that matters, but also the shape of the bubble [102]. In the case the shear forces exerted by a liquid flow exceed the surface forces, bubbles can deform $[96,103]$. The ratio of shear forces to interfacial forces is given by the capillary number $C a$ :

$$
C a=\frac{\mu u}{\sigma} .
$$


When $C a \ll 1$, interfacial forces dominate and the bubbles can assumed to be circular/spherical. For sufficiently large capillary numbers, $\mathrm{Ca}>0.1$, the bubbles can deform significantly. When the shear forces are large enough, depinning of the bubble contact lines from the corners of the gas cavities can occur [103]. Depending on the shear forces acting on the gas bubbles, eventually a continuous gas film can be obtained that acts as a lubrication layer between the liquid flow and the solid wall. As a consequence, the slip length is predicted to increase by more than one order of magnitude. The required shear forces are very large, however, which hinders experimental verification.

Superhydrophobic surfaces are subject to instabilities, as the non-wetting Cassie-Baxter state can transition to the wetting Wenzel state [18]. This increases drag forces considerably. This transition can happen suddenly when the hybrid gas-solid surface is exposed to excessive pressures, thereby exceeding the capillary pressure as given in Eq. (1.12) [104]. The transition can also be gradual, due to dissolution of the entrapped gas in the liquid (flow) [104-106].

Although often the gas-liquid surface is assumed to be free from any stress, some studies showed that this is not always the case. Bolognesi et al. [107] reported a breakdown of slippage over the air-liquid interfaces of a superhydrophobic surface with discontinuous longitudinal grooves. One of the possible explanations they give is contamination of the interface by surface-active particles or surfactants. The flow past the initially slippery interface results in accumulation of the contaminants at the downstream edge of the gas-liquid interface, leading to a surface tension gradient along the interface. This ultimately results in a Marangoni stress opposing the flow, reducing wall slip or even making the interface effectively a no-slip surface [108-110]. The experimental results of Schäffel et al. [72] confirm that adding a surfactant effectively transforms the slippery gas-liquid interface into a no-slip surface.

\subsubsection{Polymer solutions}

In the case of flow of a wide range of complex fluids such as polymer melts, solutions of (bio)polymers, suspensions and emulsions past solid surfaces, wall slip has been observed much earlier [111-115]. When considering polymer solutions, the existence of wall slip has been attributed to one of the three following slip mechanisms, although combinations of these mechanisms are also possible. First, breaking of the adhesive bonds between the polymers chains and the substrate reduces friction at the wall. This leads to adhesive wall slip. Cohesive slip, which is the second slip mechanism, occurs when the polymer chains adsorbed at the wall disentangle from those in the bulk. The third mechanism, which is considered to be the most important and relevant slip mechanism for (in particular dilute) polymeric systems $[112,114,116,117]$, is the formation of a lubrication layer between the no-slip substrate and the polymer solution. This layer is formed by 
wall depletion effects, which can be caused by for instance steric, hydrodynamic, visco-elastic and chemical forces acting between the polymer coils and the wall [112]. This results in a low-viscosity layer with thickness $\delta$ that is depleted of polymers and over which the bulk fluid flow slips. The layer thickness roughly equals the radius of gyration of the polymer [118]. The amount of slippage, which is apparent wall slip, is then expressed by Eq. (1.10): $b_{a} \sim \delta\left(\mu_{s} / \mu_{l}\right)$. In the case of viscous polymer solutions having a viscosity $\mu_{s}$ containing a low viscosity solvent with $\mu_{l}$, this expression indicates that wall slip can be significant for polymer flows.

The viscosity of complex solutions is not constant, but generally decreases with increasing shear rate $[119,120]$. This implies that flows of such shear-thinning solutions over heterogeneously slippery surfaces, which are characterised by a spatial distribution of shear at the wall $[65,66,85,92,121]$, lead to variations in the local viscosity near the wall. This will affect the overall wall slip, as for nonNewtonian fluids the relationship between shear stress and rate of deformation is not linear. This also follows from the definition of the slip condition at the wall for a power-law fluid with a viscosity described by $\mu=\mu_{0}\left(\partial_{y} u\right)^{n-1}$. The boundary condition can be written in terms of the shear stress, i.e. $E u_{b}=\mu_{0}\left(\partial_{y} u\right)^{n}$ according to Eq. (1.3), or in terms of the shear rate, i.e. $u_{b}=b \partial_{y} u$ according to Eq. (1.6). Both expressions are equivalent in the case of a Newtonian fluid, as then $b=\mu / E$, but for a non-Newtonian fluid with a power-law index of $n \neq 1$ they are not [122].

Even though complex fluids are encountered frequently in the field of microfluidics [123-125], non-Newtonian fluid flow over heterogeneously slippery surfaces has received little attention to date. We have investigated numerically the flow of inelastic, non-Newtonian aqueous xanthan gum solutions over a bubble mattress (chapter 4). These polymer solutions appear not to show slip induced by wall depletion effects [126-128]. In accordance with the analytically predicted slip enhancement, the numerical results show a significant increase in effective wall slip when operating the system in the shear-thinning regime.

\subsection{Heat and mass transfer}

\subsubsection{Concentration polarisation}

In many practical situations, exchange of heat or mass takes place between a flowing fluid and a wall. Examples are membrane systems, heat exchangers and reactors. Also in microfluidic systems, heat and mass transfer is important to understand the performance of these devices [129]. For that reason, it is desirable to predict the rate of heat or mass transfer between a fluid and a wall. As a specific example of transport in a microfluidic system, which will be discussed 
gas

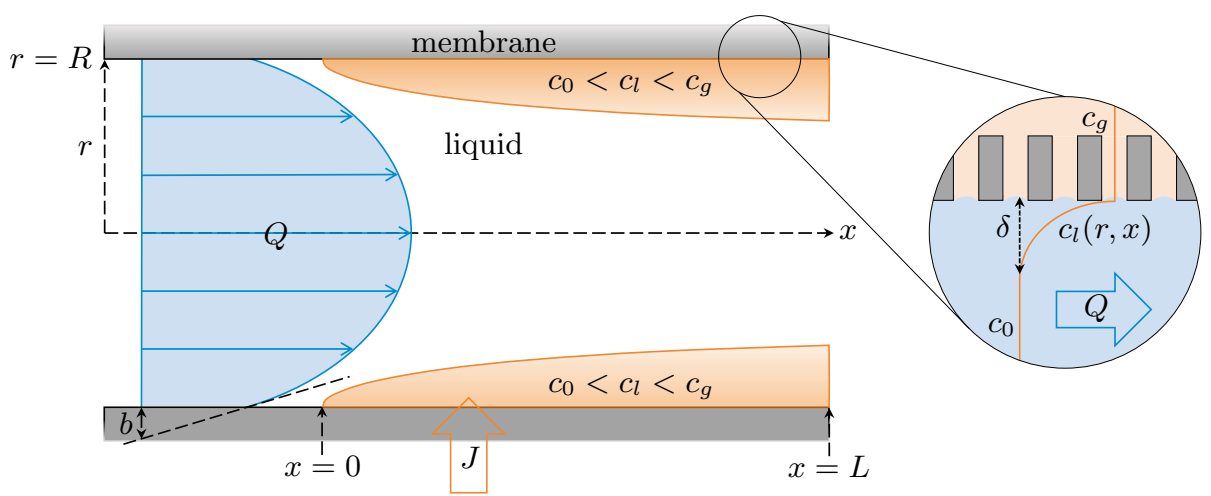

Figure 1.5 - In this system, which represents a hollow fibre membrane with a radius $R$, a gas is absorbed into a pressure-driven liquid flow via a microporous, gas-filled membrane wall. All resistance towards mass transfer is located inside the liquid, leading to concentration polarisation. The solute flux $J$ across the membrane is a function of the axial position $x$. In the boundary layer with thickness $\delta$, the solute concentration $c$ (assuming a distribution coefficient of $K_{d}=1$ ) is smaller than the gas concentration $c_{g}$, but larger than the initial concentration $c_{0}$.

later in more detail, we numerically investigated mass transfer over a slippery bubble mattress [130] (chapter 5).

The equations describing heat and mass transfer are analogous. In this section, we concentrate on systems with mass transfer. For the system shown in Fig. 1.5, the concentration at the wall of the channel is constant, implying that the resistance to mass transport is fully located in the fluid inside the channel. The concentration profile and therefore the flow-averaged concentration $\langle c\rangle$ are a function of the position in the channel. It also illustrates that the thickness $\delta$ of the boundary layer for mass transfer grows with axial position $x$. The boundary layer thickness is defined as the distance $\delta$ from the wall where the concentration has essentially reached the bulk concentration. Here, that corresponds to the point where $c_{l}=c_{0}+0.01\left(c_{g}-c_{0}\right)$, i.e. the location where the bulk concentration $c_{0}$ has been increased by $1 \%$ of the (maximum) concentration difference $\left(c_{g}-c_{0}\right)$ between the wall and the bulk [131]. In the field of membrane science and technology, the building up of such a boundary layer - the formation of concentration gradients is referred to as concentration polarisation [132, 133]. Given Fick's law stating that the solute flux $J$ equals $J=-\mathcal{D} \nabla c$, where $\mathcal{D}$ is the diffusion coefficient, it follows that the boundary layer thickness $\delta$ is related to the mass transfer coefficient $m$ by

$$
m \sim \frac{\mathcal{D}}{\delta} .
$$


Since the boundary layer is developing with position $x$, as does the flow-averaged concentration in the tube (see Fig. 1.5), it follows that the mass transfer coefficient must be a function of the axial position as well. The local mass transfer rate $\phi_{x}$ is then given by

$$
\phi_{x}=m_{x} A\left(c_{g}-\langle c\rangle\right)
$$

where $m_{x}$ is the local mass transfer coefficient between the wall with concentration $c_{g}$ and the flow-averaged solute concentration $\langle c\rangle$ in the fluid, and $A$ is the massexchanging area. The concentration difference $\Delta c=\left(c_{g}-\langle c\rangle\right)$ forms the driving force for mass transport.

The following differential equation describes mass transfer in this channel, which we assume to be cylindrical with a diameter $D$ and length $L$ :

$$
0=-Q \frac{\mathrm{d} c}{\mathrm{~d} x}+m_{x} \pi D\left(c_{g}-\langle c\rangle_{x}\right)
$$

Solving this differential equation, using the boundary conditions that $c=c_{0}$ at $x=0$ and that $c=c_{g}$ at $r=R$, yields

$$
\ln \left[\frac{c_{g}-\langle c\rangle_{x}}{c_{g}-c_{0}}\right]=-\frac{\pi D}{Q} \int_{0}^{x} m_{x} \mathrm{~d} x .
$$

The local mass transfer coefficient can be obtained from relations for the (local) Sherwood number $S h_{x}$ (or the Nusselt number $N u_{x}$, in the case of heat transfer), which is a dimensionless mass transfer coefficient that is defined as

$$
S h_{x}=\frac{m_{x} D}{\mathcal{D}} .
$$

Equivalently, the local Nusselt number $N u_{x}$ is defined as

$$
N u_{x}=\frac{h_{x} D}{k}
$$

where $h_{x}$ is the local heat transfer coefficient and $k$ is the thermal conductivity. A dimensional analysis shows that both transport numbers, which can be interpreted as the ratio of convective to diffusive heat/mass transfer, are a function of the flow conditions in the pipe, the physical properties of the fluid and the geometry of the system [131]:

$$
S h_{x}=f(R e, S c, x / L) .
$$

In the case of heat transfer, the Schmidt number $S c=\nu / \mathcal{D}$, where $\nu$ is the kinematic viscosity, needs to be replaced by the Prandtl number $\operatorname{Pr}=\nu / \alpha$, with $\alpha$ being the thermal diffusivity. The three dimensionless variables in Eq. (1.23) 


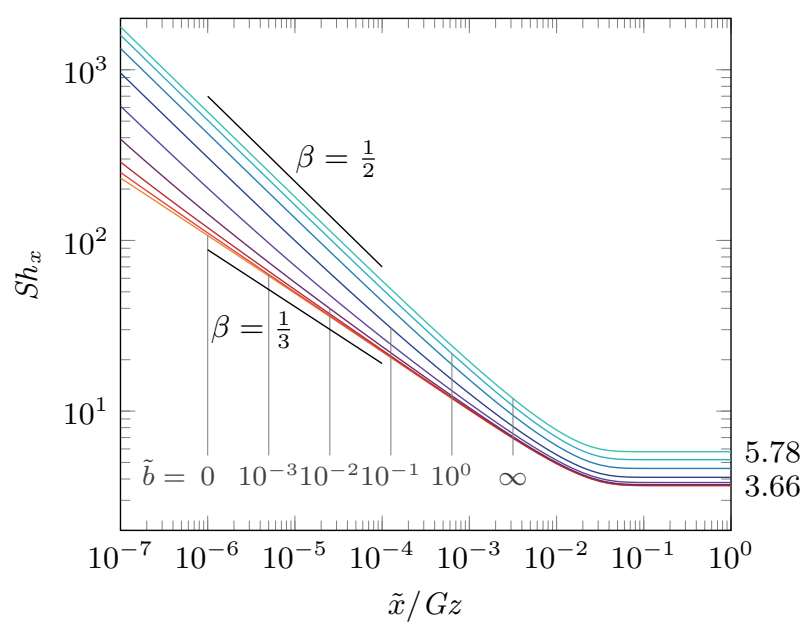

Figure 1.6 - For the problem sketched in Fig. 1.5, the local Sherwood number $S h_{x}$, which is a dimensionless mass transfer coefficient, can be obtained by solving the GraetzNusselt problem. The value of $S h_{x}$ changes with position $\tilde{x}=x / L$. The Graetz number $G z$ is defined by the physical properties of the fluid and by the system geometry. When the surface exhibits wall slip, where $\tilde{b}=b / R$, advection near the mass exchanging wall increases and consequently the Sherwood number also becomes larger [138].

are often grouped together, giving the Graetz number $G z$ :

$$
G z=\operatorname{ReSc} \frac{D}{L}
$$

\subsubsection{A classical forced convection problem}

For some systems, it is possible to obtain an analytical relationship for the Sherwood or Nusselt number by solving the advection-diffusion equation [131, 134]. In the case of a stationary system with an incompressible flow and a constant diffusion coefficient $\mathcal{D}$, mass transport is described by the following governing equation:

$$
\mathbf{u} \cdot \nabla c-\mathcal{D} \nabla^{2} c=0
$$

Under the assumption of a hydrodynamically fully developed flow, constant physical properties and negligible axial diffusion, this classical forced convection problem was first solved by Graetz [135, 136] and Nusselt [137] independently at the end of the 19th century for parabolic and uniform fluid flow through a tube with a constant wall concentration or temperature. Using the method of separation of variables, they obtained an infinite series solution for the (non-dimensionalised) concentration or temperature distribution [134]. From this solution, the local Sherwood or Nusselt number can be obtained. 
The profiles in Fig. 1.6 show that the local Sherwood number is changing with axial position for $\tilde{x} / G z<0.01$. This is the developing regime. Lévêque [139] demonstrated that in this regime, considering that in the entrance region the thermal or mass boundary layer is very thin compared to the viscous boundary layer, the Sherwood or Nusselt number obeys the following relationship:

$$
S h_{x}, N u_{x} \propto(\tilde{x} / G z)^{-\beta}
$$

The value of the exponent $\beta$ is very characteristic for the transport in the boundary layer, and it reflects that increasing the fluid velocity in the boundary layer promotes exchange of heat and mass with the wall. In the case of a parabolic velocity profile, where $\tilde{u}=2\left(1-\tilde{r}^{2}\right)$ with $\tilde{u}=u / u_{a v}$ and $\tilde{r}=r / R$ and thus with a vanishing velocity near the wall, $\beta=1 / 3$. For a uniform velocity profile, $\tilde{u}=1$, the exponent equals $\beta=1 / 2$. Barrow and Humphreys [140] found that for flows with an inverted conical or parabolic velocity profile, where the liquid velocity is largest near the wall, the heat/mass transfer rate is enhanced even further.

The developed Sherwood number $S h_{\infty}$, which is reached in the developed regime for $\tilde{x} / G z>0.1$, also increases when the velocity profile changes from parabolic to uniform. Reaching the developed regime also means that the mass boundary layer thickness exceeds the characteristic length scale of the system, which in this case means that $\delta>R$.

Since the rate of mass transport is minimum in the developed regime, preventing a system from reaching this regime promotes the overall transfer rate of mass. As follows from the Sherwood profiles in Fig. 1.6, and assuming that the geometry of a system is fixed, this can be obtained by increasing the flow rate or the Reynolds number. The Graetz number becomes larger, and therefore the maximum local Graetz number $\tilde{x} / G z$ becomes smaller. This analysis also reveals that the developed regime is reached more readily in the case of heat transfer, as generally the thermal diffusivity is much larger than the mass diffusivity (i.e. $S c \gg P r$ ).

\subsubsection{Increased convection near the wall}

Enhancing heat/mass transfer by increasing the Reynolds number is at the expense of a larger driving force or an increased energy consumption. It is for that reason that exploiting wall slip can be advantageous in two respects: for a given driving force it not only increases momentum transport, but it can also improve interfacial heat and mass transfer. This is also illustrated in Fig. 1.1: hydrodynamic slip increases the flow and therefore advection in the direct vicinity of the wall. Transport near the wall is therefore no longer driven by diffusion only, as is the case for non-slippery surfaces.

For that reason, we extended the Graetz-Nusselt problem to fluid flows with homogeneous wall slip with $0 \leq \tilde{b} \leq \infty$, where $\tilde{b}=b / R$ [138] (chapter 6). 
The resulting Sherwood profiles are plotted in Fig. 1.6, and for sufficiently large slip lengths $\left(\tilde{b}>10^{-2}\right)$ mass transport is increased in both the developing and developed regime. Thus, wall slip increases the Sherwood number and, according to Eq. (1.17), makes the boundary layer smaller. Making heat or mass exchanging surfaces slippery can therefore help to reduce the extent of concentration/temperature polarisation, which improves the performance of the process.

This study involves uniform wall slip, which is only encountered in systems with either intrinsic or apparent wall slip. In the case of intrinsic wall slip, typical slip lengths are of the order of tens of nanometres, which implies that only in nanofluidic systems heat or mass transfer can be enhanced significantly. The recently developed liquid-infused surfaces [141-144] can exhibit apparent wall slip, with the infusing liquid acting as a lubricant between the liquid-infused surface and the working liquid.

\subsubsection{Heat and mass transfer near superhydrophobic surfaces}

What about transport of heat or mass near superhydrophobic surfaces that are characterised by heterogeneous wall slip? This question was first addressed by Maynes et al. [145-148], who investigated heat transfer over superhydrophobic surfaces. They found that, due to fact that a gas has a much lower thermal conductivity than a solid, the gas phase entrapped in the surface structures can be considered as adiabatic. On the other hand, heat transfer at the solid regions of the hybrid gas-solid surface was enhanced, which they attributed to a thinner thermal boundary layer. Overall, however, the adiabatic gas patches had an adverse effect on the average Nusselt number. Only when the Péclet number $P e$ approached infinity, which (for heat transfer) is defined as

$$
P e=\operatorname{RePr}=\frac{u D}{\alpha}
$$

with $\alpha$ being the thermal diffusivity, the Nusselt number was equal to that for heat transfer over a non-slippery, solid surface. Fast fluid flow screens the influence of a heterogeneous wall on the rate of transport [149]. When accounting for the drag reduction by considering the ratio of Nusselt number to the product of friction factor $f$ to Reynold number, i.e. $N u /(f R e)$, they found that superhydrophobic surfaces do outperform non-slippery walls.

In the case of mass transfer over superhydrophobic surfaces, the boundary conditions are different. Consider a microporous membrane, with the pores filled with a gas. In that case the solid walls are impermeable and mass exchange only takes place at the slippery gas-liquid interfaces. In the case of thermal transport, the slippery gas patches are adiabatic and heat is only exchanged at the no-slip solid regions. As a consequence, heat and mass transfer over superhydrophobic 


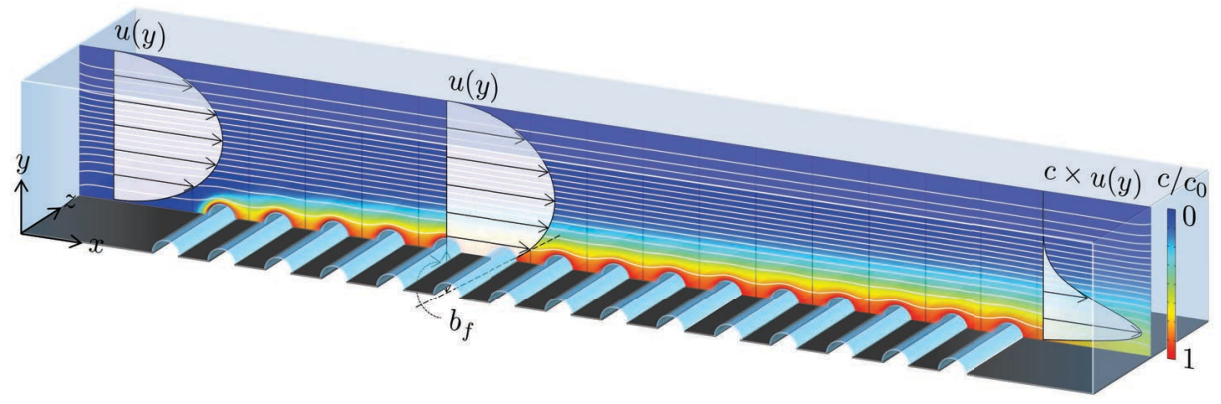

Figure 1.7 - A bubble mattress cannot only be used to reduce viscous drag, but provides also a means to enhance mass transfer. For bubbles that are, in relation to the typical channel dimension, sufficiently large and approximately flat, mass transport can be enhanced by more than $10 \%$ [130]. When the bubbles become very small, wall slip vanishes. As a result, near the wall diffusion is much faster than convection, and the wall can be treated as non-slippery and as having a uniform wall concentration.

surfaces is described by a different set of boundary conditions.

We investigated the influence of these (rather subtle) differences by extending the Graetz-Nusselt problem to heat and mass transfer over slippery, superhydrophobic surfaces [150] (chapter 7). Due to the fact that only in the case of mass transfer the locations of wall slip and mass exchange coincide, mass transfer is faster than heat transfer. Advection near the mass exchanging wall segments is larger than near the heat exchanging regions. Another important factor is the direction of the velocity component normal to the surface at the beginning of the heat/mass exchanging segments. The results also reveal that in typical microfluidic systems axial conduction cannot be neglected in the case of heat transfer, whereas for mass transfer axial diffusion only has a minor influence of the rate of transport. This again results from the fact that thermal diffusion is much faster than mass diffusion, i.e. $\operatorname{Pr} \ll S c$.

Membranes are commonly assumed to be non-slippery and to have a uniform wall concentration $[132,151]$. Therefore the classical Graetz-Nusselt and Lévêque solutions for no-slip flow are commonly used to predict the rate of mass transfer, showing good agreement with experimental data. This seems to be in contradiction with the studies discussed above, showing that wall superhydrophobicity can be used to improve transport. An example is the study of Kreulen et al. [152], who investigated the effect of the porosity of a non-wetted microporous hollow fibre membrane on the rate of carbon dioxide transport. They found that for both a surface porosity of $3 \%$ and $70 \%$, even though the pure gas was only in contact with the liquid at the pores, the active mass transfer area was equal to the total membrane area. Based on some earlier studies [149, 153-155], in which 
the influence of wall heterogeneity on mass transfer was discussed, they attributed this to the small distance between the pores compared to the tube diameter, leading to the saturation of the liquid layer near the wall with carbon dioxide.

To investigate this in more detail, we numerically studied mass transfer over a bubble mattress by assuming a fixed solute concentration at the slippery gas-liquid interfaces [130] (chapter 5). This is illustrated in Fig. 1.7. The bubble mattress can be considered as a very simple representation of a non-wetted microporous membrane surface [156]. We found that such a slippery, heterogeneous surface indeed can increase mass transfer, provided that the relative size of the bubbles is large enough. For small bubbles with size $L_{g}$, as also predicted by Eq. (1.15), wall slip and therefore convective transport near the slippery surface vanishes. As a result, diffusive transport at the scale of the bubbles is much faster than convective transport, and indeed the liquid layer near the wall is fully saturated. On larger scales - those of the channel - the wall is therefore effectively non-slippery and has a uniform wall concentration. This explains why Kreulen et al. [152], who used membranes with very small pores, did not find any influence of the surface porosity on the rate of transport, but why Karatay et al. [73, 157] reported an increase in mass transfer for slippery, structured membranes like the one shown in Fig. 1.4(a).

\subsubsection{Wall heterogeneity}

Slip is not always necessary to improve interfacial transport. Rough interfaces having a certain fractal geometry can reduce the friction towards flow, although this is based on the formation of vortices in the wall roughness at relatively high Reynolds numbers. This screens part of the no-slip walls, which can reduce flow friction [158] and/or augment heat transfer [159]. Other studies showed that roughness elements can improve heat transfer, in particular for Reynolds numbers larger than roughly $10^{2}[160,161]$. However, this enhancement is at the expense of a higher pressure drop.

\subsection{Charge transport near heterogeneous surfaces}

Heterogeneous interfaces also play an important role in charge transport. Ionexchange membranes, selective for either positively or negatively charged ions, are widely used to desalinate water by electrodialysis [162] or for power generation by for instance pressure-retarded osmosis or reverse electrodialysis [163]. Profiled membranes, which are topologically heterogeneous, can improve charge transport considerably [162, 164-167]. Also heterogeneity in terms of conductivity, arising from spatial variations in the membrane composition, can affect the performance [164, 168-170]. Recently, Davidson et al. [171] predicted numerically that surface 
Microfluidic desalination Ion-selective transport Charged hydrogels Transport mechanism

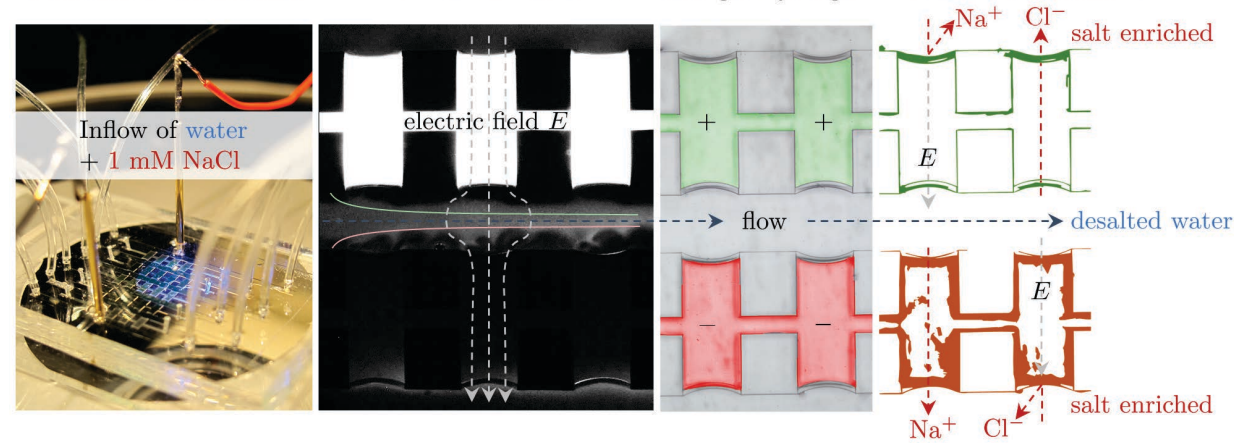

Figure 1.8 - Studying electrodialysis at the microscale can contribute to a better understanding of the transport phenomena associated with charge transfer. In this microfluidic desalination platform, cation- and anion-exchange hydrogels are patterned in situ in the polydimethylsiloxane (PDMS) chip [172]. Wall heterogeneity can promote the overall charge transport, as variations in the dielectric permittivity of the wall results in bending of the electric field lines. This induces electro-convection or the formation of vortices near the charge-selective liquid-hydrogel interfaces.

heterogeneity in terms of ion (im)permeability can accelerate charge transport through an ion-selective membrane in the overlimiting current regime significantly.

The application of an electrical potential difference $V$ over an ion-selective membrane creates a current $I$ through this membrane. Because of its ion-selectivity, this results in the formation of a concentration gradient near this membrane - a phenomenon that is referred to as concentration polarisation [173]. In the Ohmic regime, the current $I$ grows linearly with the voltage $V$. When the concentration of the transported ion near the membrane interface approaches zero, the limiting current is reached: the current does not increase with voltage anymore. In a typical IV-curve, this limiting current appears as a characteristic plateau. However, for sufficiently large voltages the current will rise again with increasing potential when the overlimiting current regime is reached. The charge or mass transfer enhancement in this regime can be, amongst other mechanisms, attributed to electro-convection $[164,174]$.

This type of convection arises when the electric field in the extended space charge region is not directed normally to the ion-selective surface. In the extended space charge region there is non-electroneutrality. This region can be larger than the electrical double layer at interfaces, which is characterised by the Debye length. Because all resistance towards charge transport is located in this charge-depleted region, very strong electric fields tangential to the surface are formed. This results in electro-convection/electro-osmosis $[173,175]$. The induced vortical fluid motion near the surface subsequently leads to additional charge transport. 
Patterning of ion-selective surfaces with impermeable regions or regions with a different dielectric permittivity causes bending of the electric field [164], and can therefore promote electro-convection, even in the Ohmic regime [171]. For larger potential differences, the heterogeneous surface promotes the formation of a regular pattern of electro-convective vortices and therewith can enhance the net charge transport. Surface patterning can also shorten the length of the limiting current density plateau [170].

Typical electrodialysis systems consist of two electrodes with a stack of alternating cation- and anion-exchange membranes placed in between [162, 163]. As illustrated in Fig. 1.8, applying a potential over such a membrane stack leads to either desalination or salt enrichment of the fluid that is flowing through the channels. To study the physical phenomena associated with desalination by electrodialysis in more detail, Kwak et al. [176, 177] have developed a microfluidic platform consisting of one or two channels in between homogeneous ion-selective membranes. Their work has provided valuable insight in these transport processes, in particular on the formation and advection of the vortices forming near the permselective membranes in the overlimiting current regime.

However, as also mentioned at the start of this section, heterogeneity influences charge transport and can even enhance it. As shown in Fig. 1.8, we developed a microfluidic platform with a stack of charge-selective hydrogels, characterised by a heterogeneous surface topology [172] (chapter 8). The in situ patterning of the hydrogels makes this platform very versatile. The transparency of the hydrogels also allows the visualisation of ion transport inside the hydrogels by using, for instance, a charge-selective dye. Although this was a proof-of-concept study and a more detailed study is required, the surface heterogeneity appears to affect the migration of the vortices under a flow and the length of the limiting current regime. This microfluidic desalination platform can therefore contribute to a better understanding of the hydrodynamic and ion transport processes that occur in (reverse) electrodialysis systems.

\subsection{Utilising hydrodynamic slip and wall heterogeneity}

Besides the drag-reducing abilities of slippery, superhydrophobic surfaces and their potential for enhancing interfacial heat and mass transfer, which both have been discussed extensively in the previous sections, these heterogeneous surfaces also have other potential applications [14, 38, 178, 179].

Typical flows in microfluidic systems are laminar, and therefore mixing only takes place by molecular diffusion. The required timescales $t$ are rather large, though, as for system with a typical length scale of $L=\mathcal{O}\left(10^{2} \mu \mathrm{m}\right)$ the residence time already should be $t \sim L^{2} / \mathcal{D}=10 \mathrm{~s}$. However, by special design of the 
microchannels $[180,181]$ or by placing ridges on the walls of a microchannel [182184], passive mixing can be promoted. In the case of ridges positioned at an oblique angle with respect to the flow direction, an anisotropic flow resistance is created that generates transverse velocity components. The resulting 'chaotic' laminar flow, resembling the generation of vortices by electro-convection in electrodialysis systems $[173,175]$, leads to mixing of streamtubes and with that increases the area across which diffusion occurs.

Wall slip can be used to improve this passive mixing, since misalignment of textured superhydrophobic surfaces also creates transverse velocity components $[80,185,186]$. Whereas the use of wetted ridges to improve mixing increases drag, superhydrophobic surfaces reduce drag. Since the difference in flow resistance parallel and perpendicular to the flow direction, which is directly related to the extent of mixing, can be enhanced using shear-free non-wetted ridges, superhydrophobic surfaces can improve passive mixing considerably [185]. The transverse velocity components generated by misaligned anisotropic superhydrophobic surfaces can also be used for sorting of microparticles with different sizes and densities [187].

Finally, wall slip can also be employed to enhance interfacially driven transport like electro-, diffusio- and thermo-osmosis [178, 188-190]. In these systems, a flow is induced by a gradient in electric potential, solute concentration or temperature in a very thin layer of thickness $\lambda$ near the interface. The friction forces between the fluid and the wall that are resisting the induced flow are alleviated when making the surface slippery. Flow enhancement factors of $(1+b / \lambda)$ have been predicted $[189,191-193]$. Since $\lambda$ can be very small, of the order of nanometres, interfacially driven transport can be enhanced considerably, even for smooth surfaces with intrinsic slip lengths that are only tens of nanometres long.

\subsection{Thesis outline}

This thesis aims to provide a better understanding on how wall slip and/or wall heterogeneity affects interfacial transport. An introduction to transport near slippery and/or heterogeneous surfaces is given in this chapter.

In chapter 2 we describe the experimental verification of the dependency of effective wall slip for transverse flow over a bubble mattress on the gas-liquid interface curvature by using micro-particle image velocity. Good qualitative and quantitative agreement between the experimental results and the numerical predictions was found.

The slip length for transverse flow over a bubble mattress is maximum for bubbles that somewhat protrude in the channel flow. By analytically and numerically analysing the spatial distribution of the viscous dissipation, we answer in chapter 3 the question of why this is the case. 
A large majority of the studies to date on slip over superhydrophobic surfaces focus on Newtonian liquids. In chapter 4 we show analytically and numerically that effective wall slip can be considerably larger for non-Newtonian liquids when operating in the shear-thinning regime.

As described in chapter 5, the heterogeneously slippery bubble mattress can be used to enhance interfacial mass transfer when compared to a non-slippery wall having a uniform wall concentration, provided that the bubbles are sufficiently large when compared to the channel height.

To generalise the effect of slippery surfaces on interfacial mass transfer, we extended the classical Graetz-Nusselt problem to homogeneously slippery surfaces. As described in chapter 6, we derived an analytical expression for the Nusselt or Sherwood number in the developing regime that is valid for any value of the slip length.

In chapter 7 we extend the Graetz-Nusselt problem to heat and mass transfer over slippery, superhydrophobic surfaces. The fact that only in the case of mass transfer the locations of wall slip and mass exchange coincide, makes mass transfer faster than heat transfer. Axial diffusion cannot be neglected in the case of heat transfer in microscale systems, whereas it has only a minor influence on the rate of mass transport.

In chapter 8 we describe the development of a microfluidic electrodialysis platform containing a stack of cation- and anion-exchange hydrogels. Under an electrical potential difference, charge is transported selectively across the heterogeneous liquid-hydrogel interfaces. The leads to either enrichment or depletion of the inflowing salt solutions. The platform can contribute to a better understanding of the various transport phenomena related to electrodialysis.

The main results of the work described in this thesis are summarised in chapter 9, followed by some suggestions for future research directions regarding slippery surfaces and their use for enhancing interfacial transport.

\section{References}

[1] S. Goldstein, in Modern developments in fluid dynamics: an account of theory and experiment relating to boundary layers, turbulent motion and wakes, edited by S. Goldstein (Dover Publications, New York, 1965), pp. $676-680$.

[2] O. Darrigol, Worlds of flow: a history of hydrodynamics from the Bernoullis to Prandtl (Oxford University Press, Oxford, 2005).

[3] C. L. M. H. Navier, Mémoire sur les lois du mouvement des fluids, Mem. Acad. Sci. Int. Fr. 6, 389 (1823). 
[4] J. D. Anderson, in The handbook of fluid dynamics, edited by R. W. Johnson (CRC press, Boca Raton, 2000).

[5] C. L. M. H. Navier, Sur les lois du mouvement des fluides, en ayant égard à l'adhésion des molecules, Ann. Chim. Phys. 19, 244 (1821).

[6] P. G. de Gennes, On fluid/wall slippage, Langmuir 18, 3413 (2002).

[7] G. H. L. Hagen, Über den Einfluss der Temperatur auf die Bewegung des Wassers in Röhren, Abh. kön. Akad. Berlin 17 (1854).

[8] G. H. L. Hagen, Über die Bewegung des Wassers in engen cylindrischen Röhren, Ann. Phys. 122, 423 (1839).

[9] J. C. Maxwell, On stresses in rarified gases arising from inequalities of temperature, Phil. Trans. R. Soc. Lond. 170, 231 (1879).

[10] C. Neto, D. R. Evans, E. Bonaccurso, H.-J. Butt, and V. S. J. Craig, Boundary slip in Newtonian liquids: a review of experimental studies, Rep. Prog. Phys. 68, 2859 (2005).

[11] E. Lauga, M. P. Brenner, and H. A. Stone, in Springer handbook of experimental fluid mechanics, edited by C. Tropea, A. L. Yarin, and J. F. Foss (Springer Berlin Heidelberg, 2007), pp. 1219-1240.

[12] C. Duez, C. Ybert, C. Barentin, C. Cottin-Bizonne, and L. Bocquet, Dynamics of fakir liquids: from slip to splash, J. Adhes. Sci. Technol. 22, 335 (2008).

[13] R. S. Voronov, D. V. Papavassiliou, and L. L. Lee, Review of fluid slip over superhydrophobic surfaces and its dependence on the contact angle, Ind. Eng. Chem. Res. 47, 2455 (2008).

[14] J. P. Rothstein, Slip on superhydrophobic surfaces, Annu. Rev. Fluid Mech. 42, 89 (2010).

[15] A. Maali and B. Bhushan, Measurement of slip length on superhydrophobic surfaces, Philos. T. R. Soc. A 370, 2304 (2012).

[16] O. I. Vinogradova and A. L. Dubov, Superhydrophobic textures for microfluidics, Mendeleev Commun. 22, 229 (2012).

[17] T. Lee, E. Charrault, and C. Neto, Interfacial slip on rough, patterned and soft surfaces: a review of experiments and simulations, Adv. Colloid Interfac. 210, 21 (2014).

[18] Y. Xue, P. Lv, H. Lin, and H. Duan, Underwater superhydrophobicity: stability, design and regulation, and applications, Appl. Mech. Rev. 68, 030803 (2016).

[19] S. Granick, Y. Zhu, and H. Lee, Slippery questions about complex fluids flowing past solids, Nat. Mater. 2, 221 (2003).

[20] E. Schnell, Slippage of water over nonwettable surfaces, J. Appl. Phys. 27, 1149 (1956).

[21] N. Churaev, V. Sobolev, and A. Somov, Slippage of liquids over lyophobic solid surfaces, J. Colloid Interf. Sci. 97, 574 (1984). 
[22] R. J. Good, Contact angle, wetting, and adhesion: a critical review, J. Adhes. Sci. Technol. 6, 1269 (1992).

[23] M. E. Schrader, Young-Dupre Revisited, Langmuir 11, 3585 (1995).

[24] D. Quéré, Wetting and Roughness, Annu. Rev. Mater. Res. 38, 71 (2008).

[25] Vladsinger, Contact angle microstates.svg (Wikimedia Commons, 2014).

[26] Y. Kazoe, K. Iseki, K. Mawatari, and T. Kitamori, Evanescent wave-based particle tracking velocimetry for nanochannel flows, Anal. Chem. 85, 10780 (2013).

[27] D. Schäffel, S. Yordanov, M. Schmelzeisen, T. Yamamoto, M. Kappl, R. Schmitz, B. Dünweg, H.-J. Butt, and K. Koynov, Hydrodynamic boundary condition of water on hydrophobic surfaces, Phys. Rev. E 87, 051001 (2013).

[28] L. Bocquet and E. Charlaix, Nanofluidics, from bulk to interfaces, Chem. Soc. Rev. 39, 1073 (2010).

[29] B. Ramos-Alvarado, S. Kumar, and G. P. Peterson, Hydrodynamic slip length as a surface property, Phys. Rev. E 93, 023101 (2016).

[30] Y. Wang and B. Bhushan, Boundary slip and nanobubble study in micro/nanofluidics using atomic force microscopy, Soft Matter 6, 29 (2010).

[31] D. Lohse and X. Zhang, Surface nanobubbles and nanodroplets, Rev. Mod. Phys. 87, 981 (2015).

[32] D. C. Tretheway and C. D. Meinhart, Apparent fluid slip at hydrophobic microchannel walls, Phys. Fluids 14, L9 (2002).

[33] D. C. Tretheway and C. D. Meinhart, A generating mechanism for apparent fluid slip in hydrophobic microchannels, Phys. Fluids 16, 1509 (2004).

[34] S. C. Hendy and N. J. Lund, Effective slip lengths for flows over surfaces with nanobubbles: the effects of finite slip, J. Phys. Condens. Mat. 21, 144202 (2009).

[35] H. Helmholtz and G. von Piotrowski, Über Reibung tropfbarer Flüssigkeiten, Sitzb. kais. Akad. Wien 40, 607 (1860).

[36] H. Brenner and V. Ganesan, Molecular wall effects: are conditions at a boundary "boundary conditions"?, Phys. Rev. E 61, 6879 (2000).

[37] G. K. Batchelor, An introduction to fluid dynamics (Cambridge University Press, Cambridge, 2000).

[38] P. Tabeling, A brief introduction to slippage, droplets and mixing in microfluidic systems, Lab Chip. 9, 2428 (2009).

[39] S. Richardson, On the no-slip boundary condition, J. Fluid Mech. 59, 707 (1973).

[40] P. Tabeling, Introduction to microfluidics (Oxford University Press, Oxford, 2011).

[41] W. Barthlott and C. Neinhuis, Purity of the sacred lotus, or escape from contamination in biological surfaces, Planta 202, 1 (1997).

[42] B. Bhushan and Y. C. Jung, Micro- and nanoscale characterization of 
hydrophobic and hydrophilic leaf surfaces, Nanotechnology 17, 2758 (2006).

[43] J. W. Bush, D. L. Hu, and M. Prakash, The integument of water-walking arthropods: form and function, Adv. Insect Physiol. 34, 117 (2007).

[44] T. Onda, S. Shibuichi, N. Satoh, and K. Tsujii, Super-water-repellent fractal surfaces, Langmuir 12, 2125 (1996).

[45] W. Chen, A. Y. Fadeev, M. C. Hsieh, D. Öner, J. Youngblood, and T. J. McCarthy, Ultrahydrophobic and ultralyophobic surfaces: some comments and examples, Langmuir 15, 3395 (1999).

[46] S. Wang and L. Jiang, Definition of superhydrophobic states, Adv. Mater. 19, 3423 (2007).

[47] F. Schellenberger, N. Encinas, D. Vollmer, and H.-J. Butt, How water advances on superhydrophobic surfaces, Phys. Rev. Lett. 116, 096101 (2016).

[48] J. Bico, C. Marzolin, and D. Quéré, Pearl drops, Europhys. Lett. 47, 220 (1999).

[49] J. Oliver, C. Huh, and S. Mason, Resistance to spreading of liquids by sharp edges, J. Colloid Interf. Sci. 59, 568 (1977).

[50] J. Kim and C.-J. Kim, Nanostructured surfaces for dramatic reduction of flow resistance in droplet-based microfluidics, Proc. IEEE Int. Conf. MEMS 479 (2002).

[51] S. Gogte, P. Vorobieff, R. Truesdell, A. Mammoli, F. van Swol, P. Shah, and C. J. Brinker, Effective slip on textured superhydrophobic surfaces, Phys. Fluids 17, 051701 (2005).

[52] A. B. D. Cassie and S. Baxter, Wettability of porous surfaces, T. Faraday Soc. 40, 546 (1944).

[53] D. Öner and T. J. McCarthy, Ultrahydrophobic surfaces. Effects of topography length scales on wettability, Langmuir 16, 7777 (2000).

[54] A. M. Peters, C. Pirat, M. Sbragaglia, B. M. Borkent, M. Wessling, D. Lohse, and R. G. H. Lammertink, Cassie-Baxter to Wenzel state wetting transition: scaling of the front velocity, Eur. Phys. J. E 29, 391 (2009).

[55] K. Watanabe, Y. Udagawa, and H. Udagawa, Drag reduction of Newtonian fluid in a circular pipe with a highly water-repellent wall, J. Fluid Mech. 381, 225 (1999).

[56] C. Cottin-Bizonne, J.-L. Barrat, L. Bocquet, and E. Charlaix, Low-friction flows of liquid at nanopatterned interfaces, Nat. Mater. 2, 237 (2003).

[57] C. Cottin-Bizonne, C. Barentin, E. Charlaix, L. Bocquet, and J. L. Barrat, Dynamics of simple liquids at heterogeneous surfaces: Molecular-dynamics simulations and hydrodynamic description, Eur. Phys. J. E 15, 427 (2004).

[58] J. Ou, B. Perot, and J. P. Rothstein, Laminar drag reduction in microchannels using ultrahydrophobic surfaces, Phys. Fluids 16, 4635 (2004).

[59] J. Ou and J. P. Rothstein, Direct velocity measurements of the flow past drag-reducing ultrahydrophobic surfaces, Phys. Fluids 17, 103606 (2005). 
[60] C.-H. Choi and C.-J. Kim, Large slip of aqueous liquid flow over a nanoengineered superhydrophobic surface, Phys. Rev. Lett. 96, 1 (2006).

[61] L. Bocquet, P. Tabeling, and S. Manneville, Comment on "Large slip of aqueous liquid flow over a nanoengineered superhydrophobic surface", Phys. Rev. Lett. 97, 109601 (2006).

[62] C.-H. Choi and C.-J. Kim, Choi and Kim reply, Phys. Rev. Lett. 97, 109602 (2006).

[63] P. Joseph, C. Cottin-Bizonne, J.-M. Benoît, C. Ybert, C. Journet, P. Tabeling, and L. Bocquet, Slippage of water past superhydrophobic carbon nanotube forests in microchannels, Phys. Rev. Lett. 97, 1 (2006).

[64] R. Truesdell, A. Mammoli, P. Vorobieff, F. van Swol, and C. J. Brinker, Drag reduction on a patterned superhydrophobic surface, Phys. Rev. Lett. 97, 044504 (2006).

[65] J. Davies, D. Maynes, B. W. Webb, and B. Woolford, Laminar flow in a microchannel with superhydrophobic walls exhibiting transverse ribs, Phys. Fluids 18, 087110 (2006).

[66] D. Maynes, K. Jeffs, B. Woolford, and B. W. Webb, Laminar flow in a microchannel with hydrophobic surface patterned microribs oriented parallel to the flow direction, Phys. Fluids 19, 093603 (2007).

[67] Y. P. Cheng, C. J. Teo, and B. C. Khoo, Microchannel flows with superhydrophobic surfaces: effects of Reynolds number and pattern width to channel height ratio, Phys. Fluids 21, 122004 (2009).

[68] C.-H. Choi, U. Ulmanella, J. Kim, C.-M. Ho, and C.-J. Kim, Effective slip and friction reduction in nanograted superhydrophobic microchannels, Phys. Fluids 18, 087105 (2006).

[69] C. Lee, C.-H. Choi, and C.-J. Kim, Structured surfaces for a giant liquid slip, Phys. Rev. Lett. 101, 064501 (2008).

[70] D. Byun, J. Kim, H. S. Ko, and H. C. Park, Direct measurement of slip flows in superhydrophobic microchannels with transverse grooves, Phys. Fluids 20, 113601 (2008).

[71] P. Tsai, A. M. Peters, C. Pirat, M. Wessling, R. G. H. Lammertink, and D. Lohse, Quantifying effective slip length over micropatterned hydrophobic surfaces, Phys. Fluids 21, 112002 (2009).

[72] D. Schäffel, K. Koynov, D. Vollmer, H.-J. Butt, and C. Schönecker, Local flow field and slip length of superhydrophobic surfaces, Phys. Rev. Lett. 116, 134501 (2016).

[73] E. Karatay and R. G. H. Lammertink, Oxygenation by a superhydrophobic slip G/L contactor, Lab Chip 12, 2922 (2012).

[74] E. Karatay, A. S. Haase, C. W. Visser, C. Sun, D. Lohse, P. A. Tsai, and R. G. H. Lammertink, Control of slippage with tunable bubble mattresses, $\mathrm{P}$. Natl Acad. Sci. USA 110, 8422 (2013). 
[75] J. R. Philip, Flows satisfying mixed no-slip and no-shear conditions, Z. angew. Math. Phy. 23, 353 (1972).

[76] J. R. Philip, Integral properties of flows satisfying mixed no-slip and no-shear conditions, Z. angew. Math. Phy. 23, 960 (1972).

[77] E. Lauga and H. A. Stone, Effective slip in pressure-driven Stokes flow, J. Fluid Mech. 489, 55 (2003).

[78] A. V. Belyaev and O. I. Vinogradova, Effective slip in pressure-driven flow past super-hydrophobic stripes, J. Fluid Mech. 652, 489 (2010).

[79] S. Schmieschek, A. Belyaev, J. Harting, and O. Vinogradova, Tensorial slip of superhydrophobic channels, Phys. Rev. E 85, 016324 (2012).

[80] J. Zhou, A. V. Belyaev, F. Schmid, and O. I. Vinogradova, Anisotropic flow in striped superhydrophobic channels, J. Chem. Phys. 136, 194706 (2012).

[81] C. Ybert, C. Barentin, C. Cottin-Bizonne, P. Joseph, and L. Bocquet, Achieving large slip with superhydrophobic surfaces: scaling laws for generic geometries, Phys. Fluids 19, 123601 (2007).

[82] A. M. J. Davis and E. Lauga, Hydrodynamic friction of fakir-like superhydrophobic surfaces, J. Fluid Mech. 661, 402 (2010).

[83] O. I. Vinogradova and A. V. Belyaev, Wetting, roughness and flow boundary conditions, J. Phys. Condens. Mat. 23, 184104 (2011).

[84] C. Cottin-Bizonne, C. Barentin, and L. Bocquet, Scaling laws for slippage on superhydrophobic fractal surfaces, Phys. Fluids 24, 012001 (2012).

[85] C. Schönecker and S. Hardt, Longitudinal and transverse flow over a cavity containing a second immiscible fluid, J. Fluid Mech. 717, 376 (2013).

[86] C. Schönecker, T. Baier, and S. Hardt, Influence of the enclosed fluid on the flow over a microstructured surface in the Cassie state, J. Fluid Mech. 740, 168 (2014).

[87] A. Busse, N. D. Sandham, G. McHale, and M. I. Newton, Change in drag, apparent slip and optimum air layer thickness for laminar flow over an idealised superhydrophobic surface, J. Fluid Mech. 727, 488 (2013).

[88] C. Schönecker and S. Hardt, Assessment of drag reduction at slippery, topographically structured surfaces, Microfluid. Nanofluid. 19, 199 (2015).

[89] S. C. Hendy and N. J. Lund, Effective slip boundary conditions for flows over nanoscale chemical heterogeneities, Phys. Rev. E 76, 066313 (2007).

[90] A. Steinberger, C. Cottin-Bizonne, P. Kleimann, and E. Charlaix, High friction on a bubble mattress, Nat. Mater. 6, 665 (2007).

[91] D. Legendre and C. Colin, Enhancement of wall friction by fixed cap bubbles, Phys. Fluids 20, 051704 (2008).

[92] C. J. Teo and B. C. Khoo, Effects of interface curvature on Poiseuille flow through microchannels and microtubes containing superhydrophobic surfaces with transverse grooves and ribs, Microfluid. Nanofluid. 17, 891 (2014).

[93] A. M. J. Davis and E. Lauga, Geometric transition in friction for flow over 
a bubble mattress, Phys. Fluids 21, 011701 (2009).

[94] C.-O. Ng and C. Y. Wang, Effective slip for Stokes flow over a surface patterned with two- or three-dimensional protrusions, Fluid Dyn. Res. 43, 065504 (2011).

[95] J. Hyväluoma and J. Harting, Slip flow over structured surfaces with entrapped microbubbles, Phys. Rev. Lett. 100, 246001 (2008).

[96] J. Hyväluoma, C. Kunert, and J. Harting, Simulations of slip flow on nanobubble-laden surfaces, J. Phys. Condens. Mat. 23, 184106 (2011).

[97] A. S. Haase, J. A. Wood, R. G. H. Lammertink, and J. H. Snoeijer, Why bumpy is better: the role of the dissipation distribution in slip flow over a bubble mattress, Phys. Rev. Fluids 1, 054101 (2016).

[98] M. Sbragaglia and A. Prosperetti, A note on the effective slip properties for microchannel flows with ultrahydrophobic surfaces, Phys. Fluids 19, 043603 (2007).

[99] D. Crowdy, Slip length for longitudinal shear flow over a dilute periodic mattress of protruding bubbles, Phys. Fluids 22, 121703 (2010).

[100] C. J. Teo and B. C. Khoo, Flow past superhydrophobic surfaces containing longitudinal grooves: effects of interface curvature, Microfluid. Nanofluid. 9, 499 (2010).

[101] D. G. Crowdy, Analytical formulae for longitudinal slip lengths over unidirectional superhydrophobic surfaces with curved menisci, J. Fluid Mech. 791, R7 (2016).

[102] X. Gu and M. Chen, Shape dependence of slip length on patterned hydrophobic surfaces, Appl. Phys. Lett 99, 063101 (2011).

[103] P. Gao and J. J. Feng, Enhanced slip on a patterned substrate due to depinning of contact line, Phys. Fluids 21, 102102 (2009).

[104] A. A. Hemeda and H. Vahedi Tafreshi, Instantaneous slip length in superhydrophobic microchannels having grooves with curved or dissimilar walls, Phys. Fluids 27, 102101 (2015).

[105] P. Lv, Y. Xue, Y. Shi, H. Lin, and H. Duan, Metastable states and wetting transition of submerged superhydrophobic structures, Phys. Rev. Lett. 112, 196101 (2014).

[106] Y. Xiang, Y. Xue, P. Lv, D. Li, and H. Duan, Influence of fluid flow on the stability and wetting transition of submerged superhydrophobic surfaces, Soft Matter 12, 4241 (2016).

[107] G. Bolognesi, C. Cottin-Bizonne, and C. Pirat, Evidence of slippage breakdown for a superhydrophobic microchannel, Phys. Fluids 26, 082004 (2014).

[108] C. Ybert and J.-M. di Meglio, Ascending air bubbles in protein solutions, Eur. Phys. J. B 4, 313 (1998).

[109] S. Tasoglu, U. Demirci, and M. Muradoglu, The effect of soluble surfactant on the transient motion of a buoyancy-driven bubble, Phys. Fluids 20, 040805 
(2008).

[110] S. Takagi and Y. Matsumoto, Surfactant effects on bubble motion and bubbly flows, Annu. Rev. Fluid Mech. 43, 615 (2011).

[111] W. Schowalter, The behavior of complex fluids at solid boundaries, J. NonNewt. Fluid Mech. 29, 25 (1988).

[112] H. A. Barnes, A review of the slip (wall depletion) of polymer solutions, emulsions and particle suspensions in viscometers: its cause, character, and cure, J. Non-Newt. Fluid Mech. 56, 221 (1995).

[113] M. M. Denn, Extrusion instabilities and wall slip, Ann. Rev. Fluid Mech. 33, 265 (2001).

[114] T. Sochi, Slip at fluid-solid interface, Polym. Rev. 51, 309 (2011).

[115] S. G. Hatzikiriakos, Slip mechanisms in complex fluid flows, Soft Matter 11, 7851 (2015).

[116] Y. Cohen and A. B. Metzner, Apparent slip flow of polymer solutions, J. Rheol. 29, 67 (1985).

[117] A. Y. Malkin, The state of the art in the rheology of polymers: achievements and challenges, Polym. Sci. Ser. A 51, 80 (2009).

[118] R. Tuinier and T. Taniguchi, Polymer depletion-induced slip near an interface, J. Phys. Cond. Mat. 17, L9 (2005).

[119] J. Blom, R. J. J. Jongschaap, and J. Mellema, Inleiding in de reologie (Kluwer Technische Boeken, Deventer, 1986).

[120] H. A. Barnes, J. F. Hutton, and K. Walters, An introduction to rheology (Elsevier, Amsterdam, 1989).

[121] E. S. Asmolov, S. Schmieschek, J. Harting, and O. I. Vinogradova, Flow past superhydrophobic surfaces with cosine variation in local slip length, Phys. Rev. E 87, 023005 (2013).

[122] G. G. Pereira, Effect of variable slip boundary conditions on flows of pressure driven non-Newtonian fluids, J. Non-Newt. Fluid Mech. 157, 197 (2009).

[123] P. Yager, T. Edwards, E. Fu, K. Helton, K. Nelson, M. R. Tam, and B. H. Weigl, Microfluidic diagnostic technologies for global public health, Nature 442, 412 (2006).

[124] P. Nghe, E. Terriac, M. Schneider, Z. Li, M. Cloitre, B. Abecassis, and P. Tabeling, Microfluidics and complex fluids, Lab Chip 11, 788 (2011).

[125] F. J. Galindo-Rosales, M. A. Alves, and M. S. N. Oliveira, Microdevices for extensional rheometry of low viscosity elastic liquids: a review, Microfluid. Nanofluid. 14, 1 (2013).

[126] S. J. Gibbs, D. Xing, T. A. Carpenter, and L. D. Hall, NMR flow imaging of aqueous polysaccharide solutions, J. Rheol. 38, 1757 (1994).

[127] S. J. Gibbs, Rheometry and detection of apparent wall slip for Poiseuille flow of polymer solutions and particulate dispersions by nuclear magnetic resonance velocimetry, J. Rheol. 40, 425 (1996). 
[128] C. J. Rofe, Nuclear magnetic resonance imaging of apparent slip effects in xanthan solutions, J. Rheol. 40, 1115 (1996).

[129] T. Gervais and K. F. Jensen, Mass transport and surface reactions in microfluidic systems, Chem. Eng. Sci. 61, 1102 (2006).

[130] A. S. Haase, E. Karatay, P. A. Tsai, and R. G. H. Lammertink, Momentum and mass transport over a bubble mattress: the influence of interface geometry, Soft Matter 9, 8949 (2013).

[131] R. B. Bird, W. E. Stewart, and E. N. Lightfoot, Transport phenomena, 2nd ed. (John Wiley \& Sons, New York, 2007).

[132] R. W. Baker, in Membrane technology and applications (John Wiley \& Sons, Ltd, Chichester, 2004).

[133] H. Strathmann, Introduction to membrane science and technology (WileyVCH, Weinheim, 2011).

[134] R. K. Shah and A. L. London, Laminar flow forced convection in ducts: a source book for compact heat exchanger analytical data (Academic Press, New York, 1978).

[135] L. Graetz, Über die Wärmeleitungsfähigkeit von Flüssigkeiten, Ann. Phys. 254, 79 (1882).

[136] L. Graetz, Über die Wärmeleitungsfähigkeit von Flüssigkeiten, Ann. Phys. 7, 337 (1885).

[137] W. Nusselt, Die Abhängigkeit der Wärmeäbergangszahl von der Rohrlänge, Z. Ver. Deut. Ing 54, 1154 (1910).

[138] A. S. Haase, S. J. Chapman, P. A. Tsai, D. Lohse, and R. G. H. Lammertink, The Graetz-Nusselt problem extended to continuum flows with finite slip, J. Fluid Mech. 764, R3 (2015).

[139] M. A. Lévêque, Les lois de la transmission de chaleur par convection, Ann. Mines Mem. Ser. 13, 201 (1928).

[140] H. Barrow and J. F. Humphreys, The effect of velocity distribution on forced convection laminar flow heat transfer in a pipe at constant wall temperature, Wärme Stoffübertrag. 3, 227 (1970).

[141] T.-S. Wong, S. H. Kang, S. K. Y. Tang, E. J. Smythe, B. D. Hatton, A. Grinthal, and J. Aizenberg, Bioinspired self-repairing slippery surfaces with pressure-stable omniphobicity, Nature 477, 443 (2011).

[142] A. Lafuma and D. Quéré, Slippery pre-suffused surfaces, Europhys. Lett. 96, 56001 (2011).

[143] B. R. Solomon, K. S. Khalil, and K. K. Varanasi, Drag reduction using lubricant-impregnated surfaces in viscous laminar flow, Langmuir 30, 10970 (2014).

[144] A. A. Hemeda and H. Vahedi Tafreshi, Liquid-infused surfaces with trapped air (LISTA) for drag force reduction, Langmuir 32, 2955 (2016).

[145] D. Maynes, B. W. Webb, and J. Davies, Thermal transport in a microchannel 
exhibiting ultrahydrophobic microribs maintained at constant temperature, J. Heat Transf. 130, 022402 (2008).

[146] D. Maynes, B. W. Webb, J. Crockett, and V. Solovjov, Analysis of laminar slip-flow thermal transport in microchannels with transverse rib and cavity structured superhydrophobic walls at constant heat flux, J. Heat Transf. 135, 021701 (2012).

[147] D. Maynes and J. Crockett, Apparent temperature jump and thermal transport in channels with streamwise rib and cavity featured superhydrophobic walls at constant heat flux, J. Heat Transf. 136, 011701 (2013).

[148] A. Cowley, D. Maynes, and J. Crockett, Effective temperature jump length and influence of axial conduction for thermal transport in superhydrophobic channels, Int. J. Heat Mass Tran. 79, 573 (2014).

[149] N. M. Juhasz and W. M. Deen, Effect of local Peclet number on mass transfer to a heterogeneous surface, Ind. Eng. Chem. Res. 30, 556 (1991).

[150] A. S. Haase and R. G. H. Lammertink, Heat and mass transfer over slippery, superhydrophobic surfaces, Phys. Fluids 28, 042002 (2016).

[151] V. Dindore, D. Brilman, and G. Versteeg, Hollow fiber membrane contactor as a gas-liquid model contactor, Chem. Eng. Sci. 60, 467 (2005).

[152] H. Kreulen, C. Smolders, G. Versteeg, and W. van Swaaij, Microporous hollow fibre membrane modules as gas-liquid contactors. Part 1. Physical mass transfer processes, J. Membrane Sci. 78, 197 (1993).

[153] K. H. Keller and T. R. Stein, A two-dimensional analysis of porous membrane transport, Math. Biosci. 1, 421 (1967).

[154] W. A. Wakeham and E. A. Mason, Diffusion through multiperforate laminae, Ind. Eng. Chem. Fund. 18, 301 (1979).

[155] W. Zhang, J. Li, G. Chen, W. You, and Z. Ren, Simulations of solute concentration profile and mass transfer behavior near the membrane surface with finite volume method, J. Membrane Sci. 355, 18 (2010).

[156] R. Wang, H. Zhang, P. Feron, and D. Liang, Influence of membrane wetting on $\mathrm{CO}_{2}$ capture in microporous hollow fiber membrane contactors, Sep. Purif. Technol. 46, 33 (2005).

[157] E. Karatay, P. A. Tsai, and R. G. H. Lammertink, Rate of gas absorption on a slippery bubble mattress, Soft Matter 9, 11098 (2013).

[158] J. Andrade, A. Araújo, M. Filoche, and B. Sapoval, Screening effects in flow through rough channels, Phys. Rev. Lett. 98, 194101 (2007).

[159] J. Andrade, E. Henrique, M. Almeida, and M. Costa, Heat transport through rough channels, Physica A 339, 296 (2004).

[160] H. Wu and P. Cheng, An experimental study of convective heat transfer in silicon microchannels with different surface conditions, Int. J. Heat Mass Tran. 46, 2547 (2003).

[161] C. Zhang, Y. Chen, and M. Shi, Effects of roughness elements on laminar 
flow and heat transfer in microchannels, Chem. Eng. Process. 49, 1188 (2010).

[162] H. Strathmann, Electrodialysis, a mature technology with a multitude of new applications, Desalination 264, 268 (2010).

[163] B. E. Logan and M. Elimelech, Membrane-based processes for sustainable power generation using water, Nature 488, 313 (2012).

[164] J. Balster, M. H. Yildirim, D. F. Stamatialis, R. Ibanez, R. G. H. Lammertink, V. Jordan, and M. Wessling, Morphology and microtopology of cation-exchange polymers and the origin of the overlimiting current, J. Phys. Chem. B 111, 2152 (2007).

[165] C. Larchet, V. Zabolotsky, N. Pismenskaya, V. Nikonenko, A. Tskhay, K. Tastanov, and G. Pourcelly, Comparison of different ED stack conceptions when applied for drinking water production from brackish waters, Desalination 222, 489 (2008).

[166] D. A. Vermaas, M. Saakes, and K. Nijmeijer, Power generation using profiled membranes in reverse electrodialysis, J. Membrane Sci. 385-386, 234 (2011).

[167] H. Strathmann, A. Grabowski, and G. Eigenberger, Ion-exchange membranes in the chemical process industry, Ind. Eng. Chem. Res. 52, 10364 (2013).

[168] I. Rubinstein, E. Staude, and O. Kedem, Role of the membrane surface in concentration polarization at ion-exchange membrane, Desalination 69, 101 (1988).

[169] J.-H. Choi, S.-H. Kim, and S.-H. Moon, Heterogeneity of ion-exchange membranes: the effects of membrane heterogeneity on transport properties, J. Colloid Interf. Sci. 241, 120 (2001).

[170] M. Wessling, L. G. Morcillo, and S. Abdu, Nanometer-thick lateral polyelectrolyte micropatterns induce macrosopic electro-osmotic chaotic fluid instabilities, Sci. Rep. 4, 4294 (2014).

[171] S. M. Davidson, M. Wessling, and A. Mani, On the dynamical regimes of pattern-accelerated electroconvection, Sci. Rep. 6, 22505 (2016).

[172] B. Gumuscu, A. S. Haase, A. M. Benneker, M. A. Hempenius, A. van den Berg, R. G. H. Lammertink, and J. C. T. Eijkel, Desalination by electrodialysis using a stack of patterned ion-selective hydrogels on a microfluidic device, Adv. Func. Mater. (2016).

[173] V. V. Nikonenko, A. V. Kovalenko, M. K. Urtenov, N. D. Pismenskaya, J. Han, P. Sistat, and G. Pourcelly, Desalination at overlimiting currents: state-of-the-art and perspectives, Desalination 342, 85 (2014).

[174] I. Rubinstein and B. Zaltzman, Electro-osmotically induced convection at a permselective membrane, Phys. Rev. E 62, 2238 (2000).

[175] N. A. Mishchuk, Concentration polarization of interface and non-linear 
electrokinetic phenomena, Adv. Colloid Interfac. 160, 16 (2010).

[176] R. Kwak, V. S. Pham, K. M. Lim, and J. Han, Shear flow of an electrically charged fluid by ion concentration polarization: scaling laws for electroconvective vortices, Phys. Rev. Lett. 110, 1 (2013).

[177] R. Kwak, G. Guan, W. K. Peng, and J. Han, Microscale electrodialysis: Concentration profiling and vortex visualization, Desalination 308, 138 (2013).

[178] H. A. Stone, A. D. Stroock, and A. Ajdari, Engineering flows in small devices: microfluidics toward a lab-on-a-chip, Annu. Rev. Fluid Mech. 36, 381 (2004).

[179] L. Bocquet and E. Lauga, A smooth future?, Nat. Mater. 10, 334 (2011).

[180] R. Liu, M. Stremler, K. Sharp, M. Olsen, J. Santiago, R. Adrian, H. Aref, and D. Beebe, Passive mixing in a three-dimensional serpentine microchannel, J. Microelectromech. S. 9, 190 (2000).

[181] C. Simonnet and A. Groisman, Chaotic mixing in a steady flow in a microchannel, Phys. Rev. Lett. 94, 134501 (2005).

[182] A. D. Stroock, S. K. W. Dertinger, A. Ajdari, I. Mezic, H. A. Stone, and G. M. Whitesides, Chaotic mixer for microchannels, Science 295, 647 (2002).

[183] T. J. Johnson, D. Ross, and L. E. Locascio, Rapid Microfluidic Mixing, Anal. Chem. 74, 45 (2002).

[184] M. A. Stremler, F. R. Haselton, and H. Aref, Designing for chaos: applications of chaotic advection at the microscale, Philos. T. R. Soc. A 362, 1019 (2004).

[185] J. Ou, G. Moss, and J. Rothstein, Enhanced mixing in laminar flows using ultrahydrophobic surfaces, Phys. Rev. E 76, 016304 (2007).

[186] T. V. Nizkaya, E. S. Asmolov, J. Zhou, F. Schmid, and O. I. Vinogradova, Flows and mixing in channels with misaligned superhydrophobic walls, Phys. Rev. E 91, 033020 (2015).

[187] E. S. Asmolov, A. L. Dubov, T. V. Nizkaya, A. J. C. Kuehne, and O. I. Vinogradova, Principles of transverse flow fractionation of microparticles in superhydrophobic channels, Lab Chip 15, 2835 (2015).

[188] J. L. Anderson, Colloid transport by interfacial forces, Ann. Rev. Fluid Mech. 21, 61 (1989).

[189] L. Bocquet and J.-L. Barrat, Flow boundary conditions from nano- to micro-scales, Soft Matter 3, 685 (2007).

[190] J. C. T. Eijkel, Liquid slip in micro- and nanofluidics: recent research and its possible implications, Lab Chip 7, 299 (2007).

[191] L. Joly, C. Ybert, E. Trizac, and L. Bocquet, Hydrodynamics within the electric double layer on slipping surfaces, Phys. Rev. Lett. 93, 257805 (2004). 
[192] L. Joly, C. Ybert, E. Trizac, and L. Bocquet, Liquid friction on charged surfaces: from hydrodynamic slippage to electrokinetics, J. Chem. Phys. 125, 204716 (2006).

[193] A. Ajdari and L. Bocquet, Giant amplification of interfacially driven transport by hydrodynamic slip: diffusio-osmosis and beyond, Phys. Rev. Lett. 96, 186102 (2006). 



\title{
CHAPTER 2
}

\section{Controlling momentum transport over a heterogeneously slippery bubble mattress}

\begin{abstract}
Control of the hydrodynamic boundary condition is not only important for studying the fundamental aspects of it, but also when applying it for drag reduction. Hydrodynamic friction on superhydrophobic substrates containing an array of slippery gas-liquid interfaces with no-slip walls in between - a bubble mattress - can potentially be optimised by controlling the interface geometry. Establishing stable and controllable gas-liquid interfaces is therefore crucial. Here, we present novel superhydrophobic microfluidic devices in which the curvature or protrusion angle of the microbubbles embedded in the wall of a hydrophobic microchannel can be controlled and stabilised. We experimentally and numerically examined the effect of the bubble geometry on the amount of wall slip. The effective slip length was obtained for a wide range of bubble protrusion angles using micro-particle image velocimetry. Our results reveal that wall slip is maximum for nearly flat bubbles, corresponding to a drag reduction of $21 \%$ at an optimal protrusion angle $\vartheta$ of approximately $10^{\circ}$. The experimental and numerical results show that the slip length decreases with increasing protrusion angles when $\vartheta>15^{\circ}$. Microfluidic devices like the ones described here provide a means to control and study the amplification of interfacial transport near slippery surfaces.
\end{abstract}

This chapter is based on the publication

E. Karatay, A.S Haase, C.W. Visser, C. Sun, D. Lohse, P.A. Tsai and R.G.H. Lammertink, Control of slippage with tunable bubble mattresses, P. Natl Acad. Sci. USA 110, 8422 (2013). 


\subsection{Introduction}

Hydrodynamic wall slip on substrates with various physicochemical properties has been subject of scientific research for more than two decades [1-8]. Tuning hydrodynamic slippage remains a challenge, however, in particular for laminar flow in microfluidic devices. The amount of slippage is usually quantified by a slip length, which corresponds to the distance below the surface where the linearly extrapolated velocity profile would equal zero again $[6,7]$. This slip length ranges from a few nanometres for flat hydrophobic surfaces to tens of micrometres for superhydrophobic substrates with hybrid (gas-liquid and liquid-solid) interfaces [6-8]. Superhydrophobic surfaces in the Cassie-Baxter state are characterised by a (nearly) non-slippery hydrophobic wall containing nano- or microstructures, which are typically posts, grooves or cavities, in which slippery gas bubbles are entrapped. These surfaces have been shown to be advantageous for drag reduction [9-15]. Because of their heterogeneous nature, slippage over superhydrophobic surfaces is characterised by an effective slip length [7].

An important parameter determining the amount of slip is the orientation of the surface structures with respect to the flow direction, which can be perpendicular, parallel or oblique $[10,16-18]$. The geometry of the gas-liquid menisci also influences the effective slip length $[15-17,19,20]$. In particular for bubbles positioned transversely to the flow direction, the interface curvature affects the flow resistance. The curvature is characterised by the so-called protrusion angle $\vartheta$ of the bubbles. When these bubbles protrude strongly in the flow, a transition from slippage to friction has been predicted theoretically $[17,21]$ and numerically [16, 19, 22]. By using a surface force apparatus, Steinberger et al. [19] demonstrated experimentally that friction is increased for flow over a hydrophobic surface containing trapped gas bubbles with a radius of $1.3 \mu \mathrm{m}$ and a large protrusion angle in the range of $30^{\circ}$ to $60^{\circ}$.

The existence of a critical protrusion angle suggests that the flow resistance can be manipulated via the bubble geometry. However, there has been no experimental investigation of flow over a superhydrophobic surface with embedded microbubbles positioned transversely to the flow - often referred to as a bubble mattress - for a wide range of protrusion angles. Here, we report on integrated glass-silicon microfluidic devices in which stable and controllable gas bubbles at the wall of the hydrophobic microchannels under a pressure-driven flow could be realised. The flow fields were measured using micro-particle image velocimetry for various protrusion angles. Using these experimentally determined velocity fields, we examine in detail the effect of the bubble geometry and surface porosity on the amount of effective wall slip. 

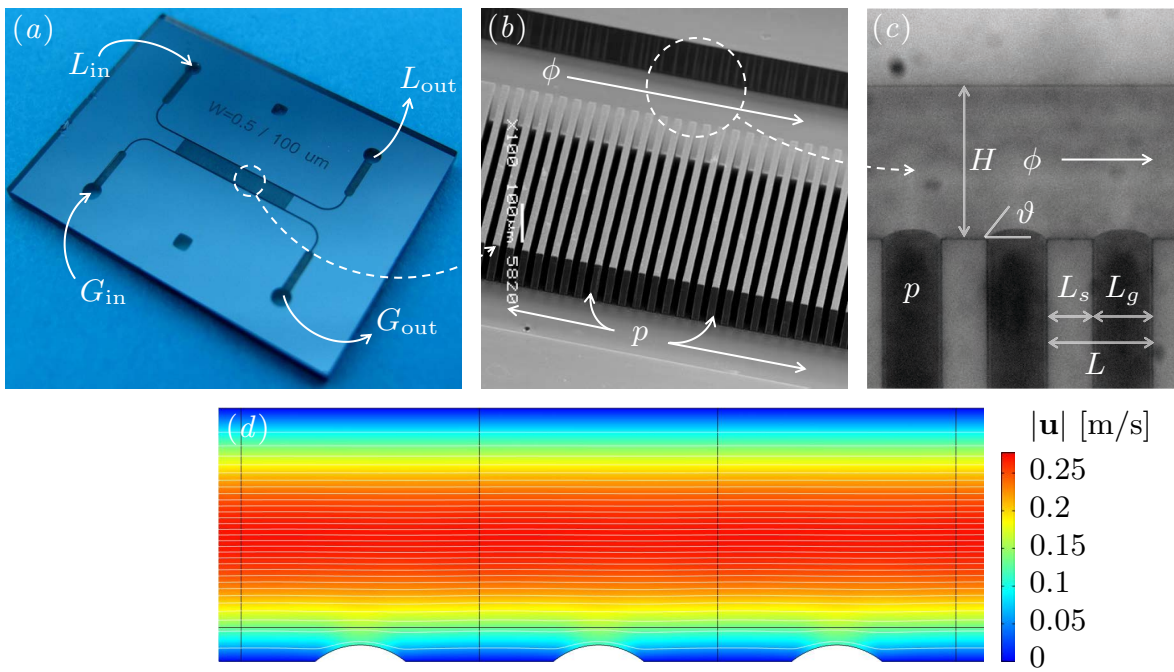

Figure 2.1 - (a) Microfluidic devices were fabricated to form a stable and controllable bubble mattress. (b) The chips consisted of two parallel main channels of height $H$, which were connected by an array of side channels in between. (c) Hydrophobisation of the channel walls allowed the formation of a stable bubble mattress. By varying the nitrogen gas pressure $p$, the bubble protrusion angle $\vartheta$ could be controlled. Devices with two different porosities $\varepsilon=L_{g} / L$ were used to investigate the influence of the protrusion angle on the amount of slip in the channels. $(d)$ To validate the experimental results, a numerical model was built that resembled the experimental devices.

\subsection{Materials and methods}

\subsubsection{Fabrication of microfluidic devices}

To create a bubble mattress in a chip, we designed and fabricated microfluidic devices that consisted of two parallel main channels, connected by an array of side channels in between (see Fig. 2.1). To prevent the side channels from wetting, the initially hydrophilic glass/silicon channels walls were hydrophobised using perfluorinated octyltrichlorosilane (FOTS) as a hydrophobisation agent [23]. For sufficiently large nitrogen gas pressures $p$, the bridging side channels were filled with gas. By flowing water through one of the main channels, a bubble mattress was formed. The protrusion angle $\vartheta$ of the gas bubbles, which were pinned at the corners of the side channels with the water-filled main channel, could be controlled by changing the gas pressure.

The microchannels were fabricated by standard photo-lithography followed by deep reactive ion etching of the silicon wafers. The microchannels were sealed by anodic bonding of the processed silicon wafers to glass wafers. The depth of the microchannels was approximately $100 \mu \mathrm{m}$. The height $H$ of the main microchannels was $50 \mu \mathrm{m}$. The width (or projected diameter) $L_{g}$ of the gas-filled 
side channels was $20 \mu \mathrm{m}$. To vary the porosity or bubble surface coverage $\varepsilon=L_{g} / L$ of the devices, the length $L_{s}$ of the solid walls between two side channels was varied. The periodic bubble unit length $L$ is then defined as $L=L_{g}+L_{s}$. The effective slip length was experimentally quantified for two different shear-free fractions: $\varepsilon=0.38$ and $\varepsilon=0.54$. The water flow rate $\phi$ was fixed at $\phi=45 \mu \mathrm{L} / \mathrm{min}$. We operated at sufficiently small capillary numbers of $C a=\tau_{w} L_{g} / \sigma<10^{-2}$ to ensure that the gas bubbles were spherical. Here, $\tau_{w}$ is the shear stress imposed by the liquid on the hybrid wall and $\sigma$ is the interfacial tension of the nitrogen-water interface.

\subsection{2 $\mu$ PIV experiments}

The flow field for a steady, laminar flows was experimentally determined by microparticle image velocimetry $(\mu \mathrm{PIV})$. An inverted microscope equipped with a $40 \times(\mathrm{NA}=0.75)$ objective formed the basis of the experimental setup. The two-dimensional velocity profile $\mathbf{u}=\left(u_{x}, u_{y}\right)$ was measured in the middle of the microchannel, i.e. in the middle between the glass top wall and the silicon bottom wall. The $\mu$ PIV correlation depth is estimated to be $3.1 \mu \mathrm{m}$. Milli-Q water with a density $\rho$ and a dynamic viscosity $\mu$, seeded with 0.1 vol\% red fluorescing polystyrene particles of $1 \mu \mathrm{m}$ diameter, was used as the working fluid. A dual cavity Nd:YAG laser with a wavelength of $532 \mathrm{~nm}$ was used to illuminate the channel. The image pairs with a delay time of $\Delta t=7 \mu$ s between two exposures were recorded using a 12 bit PCO Sensicam qe 670KD double-shutter CCD camera with a resolution of $1376 \times 1040$ pixels. To enhance the particle visibility and the signal-to-noise ratio of the correlation map, image pre-processing was performed before cross-correlation. Averaged mean intensity images were calculated and subtracted from the image pairs. The particle image density was artificially increased by using a consecutive sum of five images in a row to increase the resolution of the final vector field. The interrogation window of the $\mu \mathrm{PIV}$ images was $222 \times 167 \mu \mathrm{m}^{2}$. A multi-grid ensemble correlation averaging method was employed to calculate the flow field from 195 image pairs. When processing the data, the interrogation window size was decreased in three steps to a size of $32 \times 16$ pixels, corresponding to a window size of $5.2 \times 2.6 \mu \mathrm{m}^{2}$, with a $50 \%$ overlap of the interrogation windows. In this way we could achieve vector fields consisting of $39 \times 79$ velocity vectors at high spatial resolution. Since our data had high signal-to-noise ratios, no smoothing needed to be applied to the velocity fields. For each measurement, bright-field images were captured to define the locations of the bubbles and the channel walls on the raw $\mu$ PIV images. The average bubble protrusion angle was determined by employing DropSnake [24], a plug-in for ImageJ [25]. 


\subsubsection{Numerical procedure}

To validate the experimental results, we also numerically investigated the influence of the interface geometry on the amount of effective slip using COMSOL Multiphysics 4.1. The two-dimensional model, which resembled the experimental devices, consisted of 15 successive bubble units and an entrance/outlet domain of length $2 L$. The bubbles were described as rigid spherical arcs with a protrusion angle $\vartheta$ and a projected diameter $L_{g}$. The gas-liquid interface curvature was parameterised by the bubble protrusion angle $\vartheta$. A no-slip boundary condition was applied to the solid walls, whereas the bubble surfaces were described as shear-free walls. The pressure-driven flow field was calculated for both $\varepsilon=0.38$ and $\varepsilon=0.54$ using an average velocity of $u_{a v}=0.182 \mathrm{~m} / \mathrm{s}$ and $u_{a v}=0.204 \mathrm{~m} / \mathrm{s}$, respectively, as obtained from the experimentally determined vector fields.

\subsubsection{Calculation of effective slip length}

The amount of wall slip can be quantified by a slip length $b$, which is often defined by Navier's slip condition [26],

$$
u_{b}=\left.b \frac{\partial u_{x}}{\partial y}\right|_{y=0}
$$

where $u_{b}$ is the slip velocity, and $\partial_{y} u_{x}$ is the velocity gradient normal to the wall at $y=0$. In the case of heterogeneously slippery systems, like the bubble mattress considered here, the local amount of slip varies. In that case, a system is characterised by the effective slip length $b_{f}$, which is the slip length required to let a homogeneous slip flow have the same average velocity and pressure gradient as a flow over a heterogeneously slippery surface. For slit flow, on can derive that the effective slip length is described by

$$
b_{f}=\left(-u_{a v}-\frac{H^{2}}{12 \mu} \frac{\mathrm{d} p}{\mathrm{~d} x}\right) /\left(-\frac{u_{a v}}{H}-\frac{H}{3 \mu} \frac{\mathrm{d} p}{\mathrm{~d} x}\right) .
$$

Experimentally, however, the pressure gradient is not known. In that case the effective slip length needs to be obtained from the measured flow field. Commonly, when using $\mu \mathrm{PIV}$ to quantify wall slip, the slip length is obtained by linear extrapolation of the velocity profile near the wall $[5,14,15,27,28]$. Here, we assumed that close to the wall the velocity component in the $x$-direction depends linearly on the $y$-coordinate, i.e. $u_{x}(y) \propto y$. The shear rate $\partial_{y} u_{x}$ at the wall is obtained by a least-squares linear fitting to the five velocity components $u_{x}$ adjacent to the solid wall or the bubble surface. The fitted velocity profile allows direct computation of the local slip length $b_{l}(x)$ following Eq. (2.1), although the shear rate is evaluated using data points for which $y>0$. Note that there are also other methods to compute the slip length; see the appendix to this chapter 
for a brief discussion.

Since we found local slip lengths that are considerably smaller than the bubble unit length, i.e. $b_{l}<L$, the effective slip length $b_{f}$ can be obtained by averaging the local slip lengths $b_{l}$ for a given protrusion angle [29]:

$$
b_{f}=\frac{1}{n} \sum_{i=1}^{n} b_{l}(i)
$$

To allow comparison with the experimental results, the effective slip length $b_{f}$ in the numerical simulations was obtained from the average velocity $\left\langle u_{x}\right\rangle$ and shear rate $\left\langle\partial_{y} u_{x}\right\rangle$ at a fixed height $h$ according to the equation

$$
b_{f}=\left.\frac{\left\langle u_{x}\right\rangle}{\left\langle\partial u_{x} / \partial y\right\rangle}\right|_{y=h}-h .
$$

The maximum protrusion depth of the gas bubbles in the $y$-direction determines the minimum value of $h$. As this depth equals $6.4 \mu \mathrm{m}$ for a protrusion angle of $\vartheta=65^{\circ}$, the height $h$ was set to $h=6.5 \mu \mathrm{m}$. Both $\left\langle u_{x}\right\rangle$ and $\left\langle\partial_{y} u_{x}\right\rangle$ at $y=h$ are the average values for the middle 11 bubble units, where the flow is hydrodynamically developed and periodic.

\subsubsection{Calculation of friction factor}

To illustrate the capability of actively controlling the amount of wall slip in our microfluidic devices by changing the bubble protrusion angle, the effective slip length is rewritten in terms of the Fanning friction factor $f$. This friction factor is defined as $f=2 \tau_{w} / \rho u_{a v}^{2}[30,31]$, with $\tau_{w}$ being the shear stress at the wall. In the case of flow through a slit of height $H$ and length $\Delta x, H \Delta p=2 \tau_{w} \Delta x$. We then find for the Fanning friction factor

$$
f=\frac{H}{\rho u_{a v}^{2}}\left|\frac{\Delta p}{\Delta x}\right| .
$$

The overall pressure gradient $\Delta p / \Delta x$, which equals the pressure gradient $\mathrm{d} p / \mathrm{d} x$ in the case of a homogeneous slip flow, can be expressed in terms of the effective slip length $b_{f}$ by rewriting Eq. (2.2) as

$$
\left|\frac{\Delta p}{\Delta x}\right|=\frac{12 \mu u_{a v}}{H^{2}} \frac{\left(b_{f}+H\right)}{\left(4 b_{f}+H\right)} .
$$

Substitution yields the final expression for the friction factor $f$ :

$$
f=\frac{12 \mu}{\rho u_{a v} H} \frac{\left(b_{f}+H\right)}{\left(4 b_{f}+H\right)}=\frac{12}{R e} \frac{\left(b_{f}+H\right)}{\left(4 b_{f}+H\right)},
$$


where $R e=\rho u_{a v} H / \mu$ is the Reynolds number. Since the average velocity and therefore the Reynolds number varied from experiment to experiment, we normalised the friction factor using the value for $f=f_{0}=12 / R e$ when $b_{f}=0$. This normalised friction factor reads as

$$
\frac{f}{f_{0}}=\frac{b_{f}+H}{4 b_{f}+H}
$$

\subsection{Results and discussion}

\subsubsection{Measuring the flow field}

The design of the microfluidic devices allowed active control of the gas-liquid interface curvature and enabled visualisation of both the interface geometry and the flow field near the gas bubbles. The experimental results presented here were obtained using two chips with a different porosity. In Fig. 2.2 two examples are shown of a flow field determined using micro-particle image velocimetry. The vector fields are plotted on top of the bright field images of the corresponding experiments, clearly showing the bubbles being pinned at the sharp corners of the side channels. Since the capillary number was small, $C a<10^{-2}$, the shear forces were much smaller than the capillary forces. The nitrogen-water interface could therefore be considered as spherical, which is important regarding the slipperiness of the gas-liquid interface [22, 32]. The local balance between gas and liquid pressure determined the interface curvature. We were able to vary the bubble protrusion angle $\vartheta$ in the range of $-2^{\circ}<\vartheta<43^{\circ}$ by slightly changing the gas pressure. Whereas conventional superhydrophobic surfaces are unstable due to dissolution of the entrapped gas in the liquid flow [15, 33-35], the active supply of nitrogen in our devices ensured that the bubbles were stable during the experiment.

The presence of a heterogeneously slippery bottom wall affected the local properties of the flow field. Fig. 2.3( $a$ ) shows that above the bubbles the maximum velocity increased significantly. Comparison with the numerically calculated velocity profiles shows that this can be attributed to the confinement effect of the bubbles protruding into the channel. For (nearly) flat bubbles, on the other hand, the maximum velocity above the bubbles was slightly smaller than above the no-slip wall segments. The slippery bubbles reduced friction at the wall, which, since the flow rate is fixed, led to a flattening of the velocity profile.

The flow in $y$-direction also depends on the protrusion angle. Consider for instance the bubble located at $70<x<90 \mu \mathrm{m}$ (see Fig. 2.2(a)). At the start of the bubble, for $x<80 \mu \mathrm{m}$, Fig. 2.3(b) shows that the liquid flowed towards the bubble surface when the bubble was flat, as $u_{y}<0$. However, Fig. 2.3(c) reveals that when the protrusion angle was large enough, the flow direction was towards 
(a) $\varepsilon=0.38, \vartheta=35.4^{\circ}, L_{g}=18.9 \mu \mathrm{m}, H=48.7 \mu \mathrm{m}$

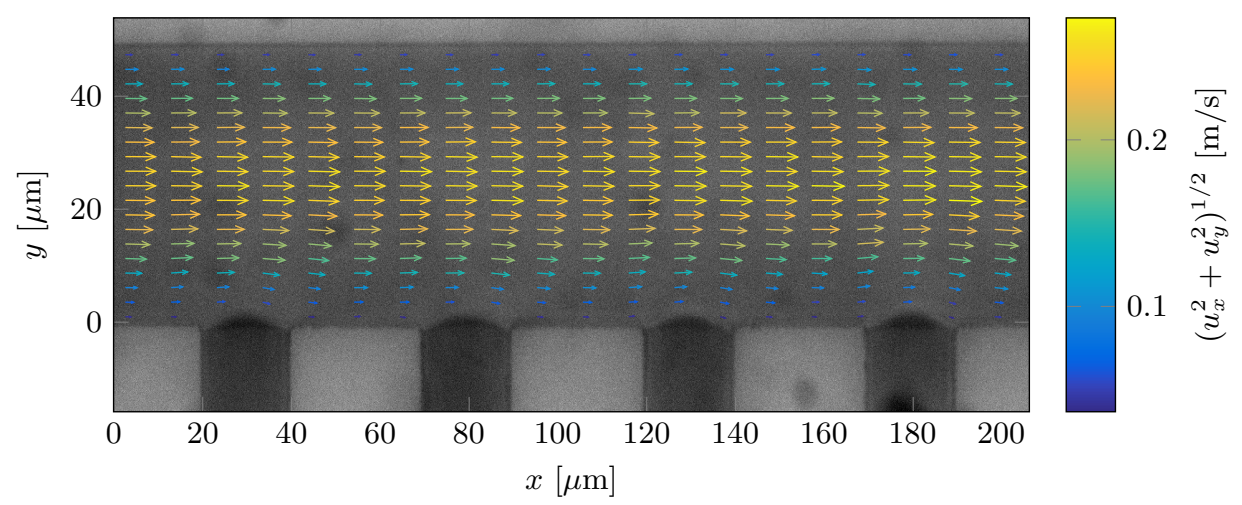

(b) $\varepsilon=0.54, \vartheta=23.6^{\circ}, L_{g}=21.6 \mu \mathrm{m}, H=51.4 \mu \mathrm{m}$

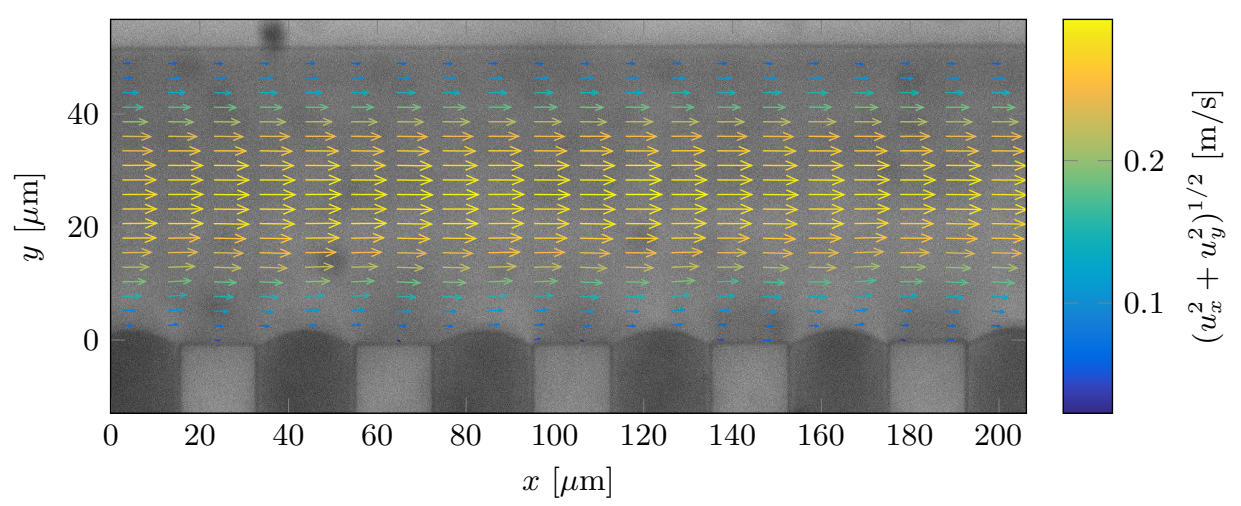

Figure 2.2 - This figures shows the vector fields for $(a)$ a surface porosity of $\varepsilon=0.38$ and a protrusion angle of $\vartheta=35.4^{\circ}$ and for $(b) \varepsilon=0.54$ and $\vartheta=23.6^{\circ}$. The bright field images were used to determine the value of $\vartheta$ and show that the bubbles were pinned at the corners of the side channels. Velocity vector that are located inside the bubbles were omitted in the calculation of the slip lengths.

the middle of the channel, i.e. $u_{y}>0$. At the end of the bubble, for $x>80 \mu \mathrm{m}$, the situation was reversed. Numerical calculations show that the direction near the front/back edge of a bubble surface reverses for a protrusion angle around $30^{\circ}$, which corresponds with the velocity profiles shown in Fig. 2.3.

The streamwise velocity components $u_{x}(x, y)$ for $\vartheta=35.4^{\circ}$ and $\varepsilon=0.38$ are plotted in Fig. 2.4 for different values of $y$. These $y$-values are indicated on top of a raw $\mu \mathrm{PIV}$ image. The channel walls and bubble surfaces are indicated by the solid white lines. The presence of the gas bubbles had a strong effect on the details of the flow field. The variations in this field reflect the change 
(a) $|\mathbf{u}|$ for $\vartheta=35.4^{\circ}$

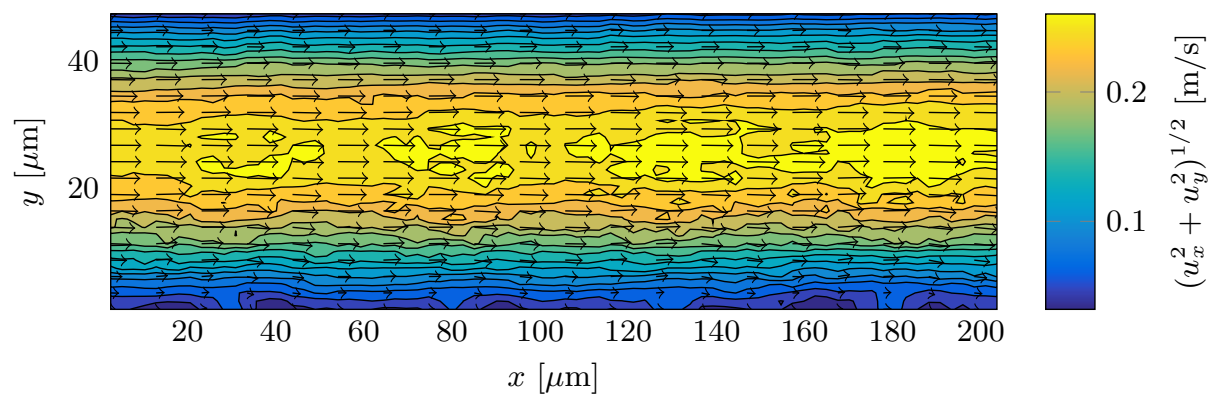

(b) $u_{y}$ for $\vartheta=1.2^{\circ}$

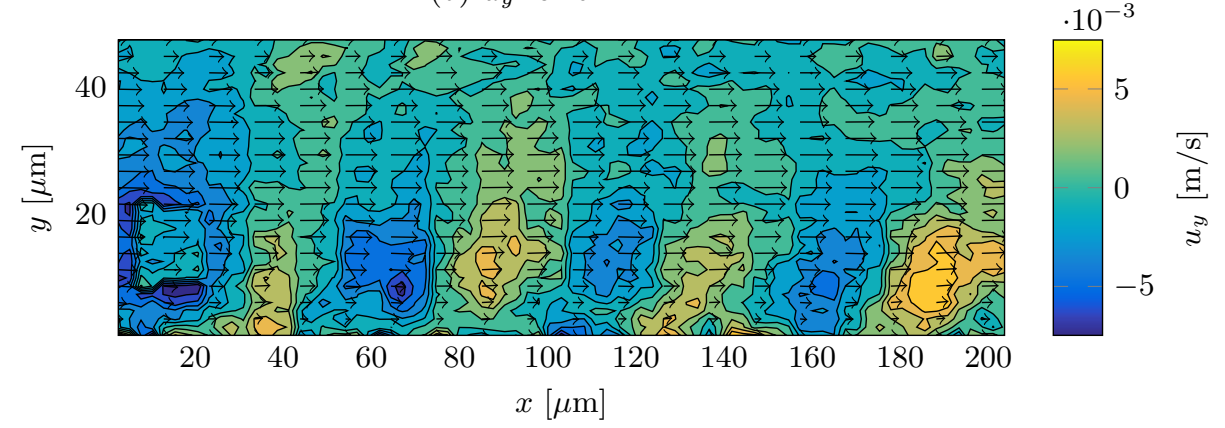

(c) $u_{y}$ for $\vartheta=35.4^{\circ}$

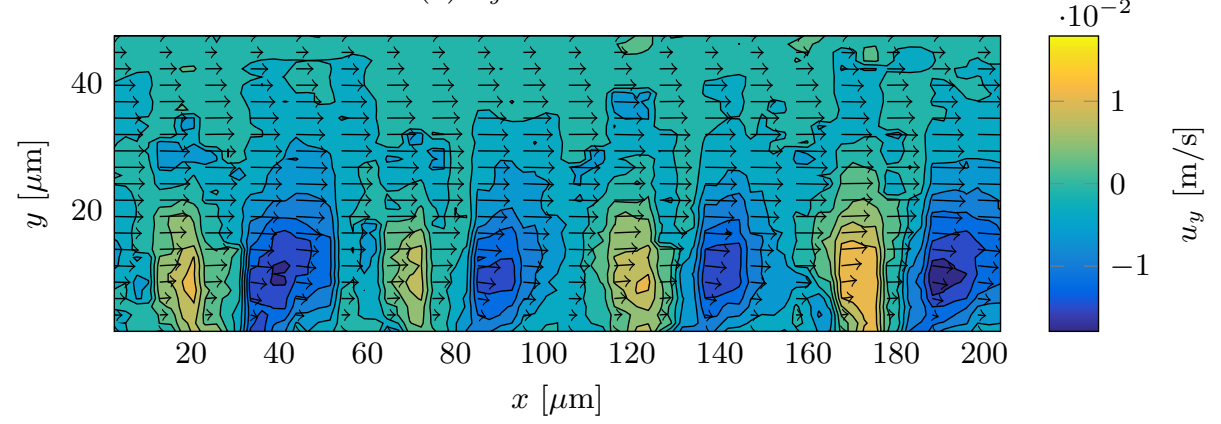

Figure 2.3 - The experimentally determined flow fields reveal how the heterogeneity of the bottom wall affected the flow field $\mathbf{u}=\left(u_{x}, u_{y}\right)$. All figures were obtained using the chip with a porosity of $\varepsilon=0.38$. For sufficiently large protrusion angles, the liquid velocity locally increased, as the plot in $(a)$ shows. The value of the protrusion angle $\vartheta$ also determined the $y$-directional velocity. At the start of a bubble, $u_{y}$ was negative in the case of $(b)$ flat bubbles and positive when $\vartheta$ was $(c)$ larger than approximately $30^{\circ}$.

in hydrodynamic boundary condition at the hybrid bottom wall. Even at an height of $y=7.4 \mu \mathrm{m}=0.15 \mathrm{H}$, the flow field was affected by the presence of 


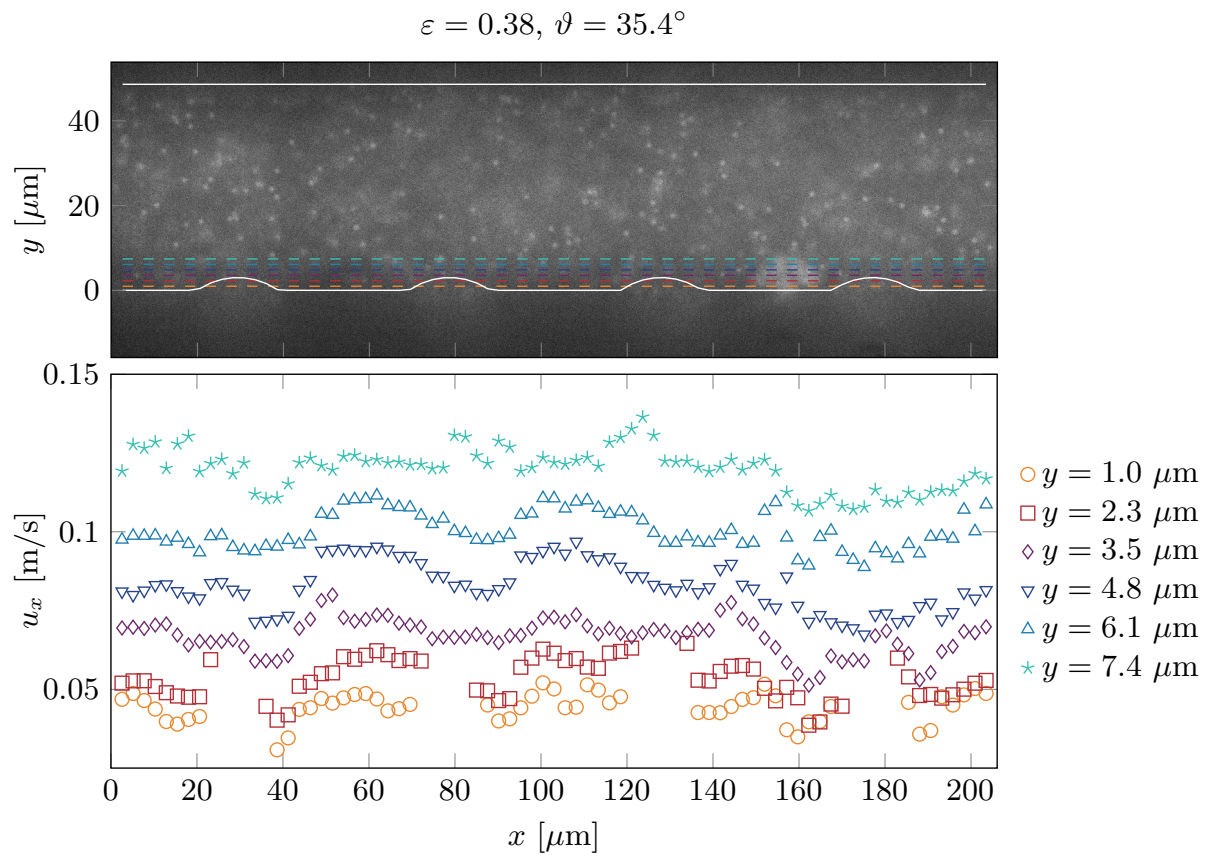

Figure 2.4 - Streamwise velocity component $u_{x}$ for $\varepsilon=0.38$ and $\vartheta=35.4^{\circ}$. The $y$-location of the velocity components is indicated on top of the raw $\mu \mathrm{PIV}$ image. The white lines indicate the position of the walls and the bubble surfaces.

the bubbles that protruded $3.0 \mu \mathrm{m}$ in the channel. Although the effective slip length is positive for a protrusion angle of $\vartheta=35.4^{\circ}$, near the wall the protruding bubbles formed an obstacle to the flow: a slight deceleration was observed in front of the bubbles. Behind the bubble, the loss in velocity was recovered and $u_{x}$ increased again. These observations are consistent with the numerical results of Hyväluoma et al. [16]. Because the bubbles protruded into the main channel, the $u_{x}(x)$-profiles for in this case $y<3.0 \mu \mathrm{m}$ are discontinuous at the location of the bubbles. The velocity vectors that are located inside the bubbles were also excluded from the calculations of the effective slip lengths.

\subsubsection{Effective wall slip}

The effective slip length for a given protrusion angle is obtained by averaging the local slip lengths, which were obtained by fitting a straight line to the five data points adjacent to the solid wall or bubble surface. This procedure is illustrated in Fig. 2.5, depicting a fit to the average $x$-directional velocity profile $\left\langle u_{x}\right\rangle(y)$. The distance between the surface and the point $y$ where the (fitted) velocity profile equals zero corresponds to the slip length. As Fig. 2.5 shows, the variation in 


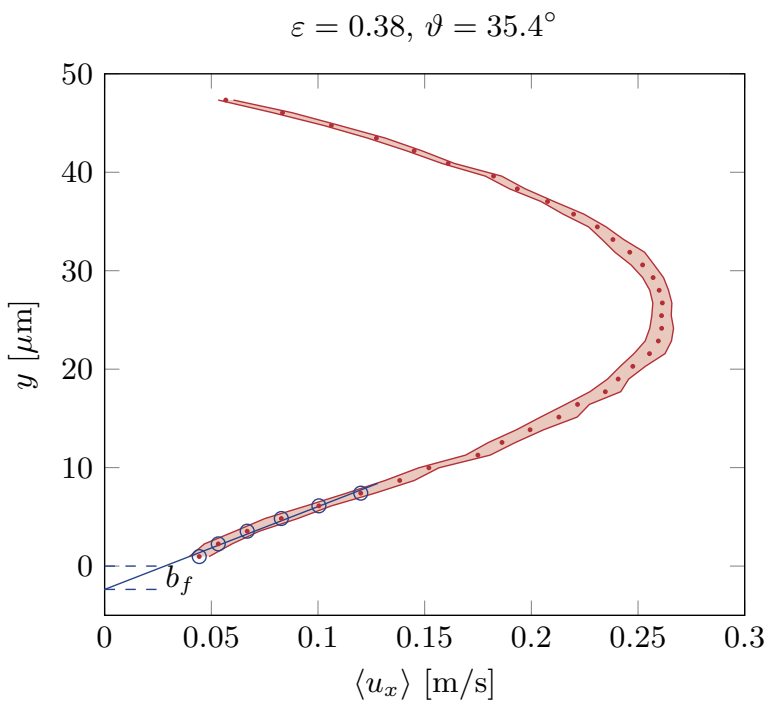

Figure 2.5 - Average velocity profile $\left\langle u_{x}\right\rangle(y)$, with the colour band indicating the standard error of the mean for $\left\langle u_{x}\right\rangle$ for a given position $y$. The effective slip lengths shown in Fig. 2.6 are obtained by averaging the local slip lengths $b_{l}$, which were obtained by fitting a straight line to the five values of $u_{x}(x, y)$ adjacent to the solid wall or the bubble surface. This figure is therefore only an illustration of how the slip length is determined.

$u_{x}$ is larger in the bottom half of the channel $(0<y<0.5 H)$ than near the top wall at $y=H$. This also corresponds with Fig. 2.3(a) and Fig. 2.3(c). The influence of the wall heterogeneity and the protruding bubbles on the flow profile propagates deeply into the main channel.

In Fig. 2.6 we show the experimentally obtained effective slip lengths $b_{f}$ plotted as a function of the bubble protrusion angle $\vartheta$ for two different surface porosities $\varepsilon$. Next to the experimental values for $b_{f}$, also the numerically calculated slip length profiles are plotted. Fig. 2.6 shows that the experimental results and numerical predictions are in good agreement, both qualitatively and quantitatively. There exists a clear dependency of the slip length on the geometric parameters $\varepsilon$ and $\vartheta$. For $\varepsilon=0.54$, the maximum effective slip length was $b_{f}=5.2 \pm 0.4 \mu \mathrm{m}$ for a protrusion angle of $\vartheta=12^{\circ} \pm 2^{\circ}$. For larger protrusion angles, the slip length decreased. The protruding bubbles increasingly formed obstacles to the fluid flow, and the flow resistance increased. This is in agreement with the numerically obtained slip lengths. Although the experimental data for $\varepsilon=0.38$ is more scattered, in particular for small $\vartheta$, the general trend is the same: wall slip is maximum for flat bubbles, i.e. for $0^{\circ}<\vartheta<15^{\circ}$, but decreases when the bubbles protrude more into the channel flow.

The error in the slip length originates mainly from the uncertainty regarding 


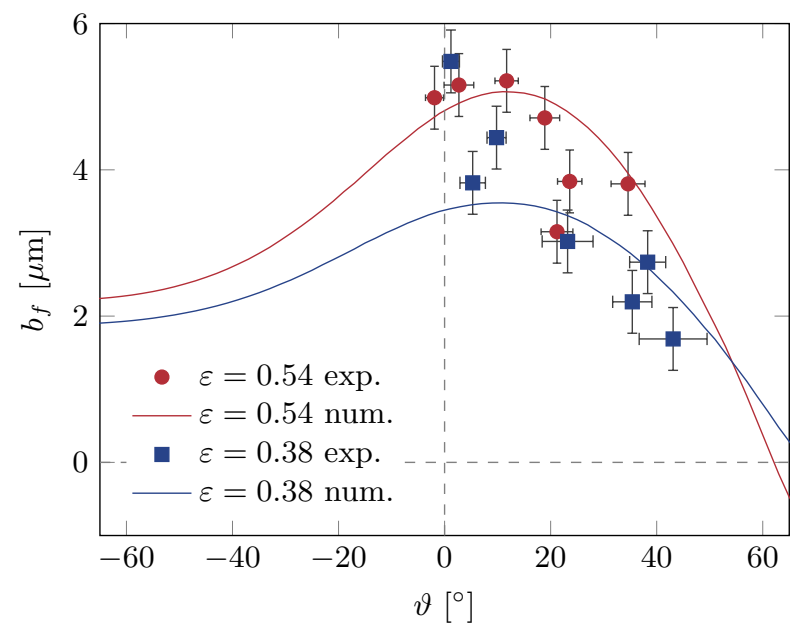

Figure 2.6 - Effective slip length $b_{f}$ as a function of the bubble protrusion angle $\vartheta$ for two different surface porosities $\varepsilon$, obtained by $\mu \mathrm{PIV}$ experiments (symbols) and numerical calculations (lines). The slip lengths are calculated by fitting a straight line to the five velocity vectors adjacent to the wall.

the position of the wall. This position directly affects the value of the slip length. Consider for instance the velocity profile for $\left\langle u_{x}\right\rangle(y)$ in Fig. 2.5. The channel height is determined to be $H=48.7 \mu \mathrm{m}$, whereas the profile suggests that only zero liquid velocity is reached when $y \approx 50 \mu \mathrm{m}$. It is known, though, that velocity measurements near walls using $\mu \mathrm{PIV}$ are biased due to particle depletion and strong velocity gradients $[36,37]$. As a result, the liquid velocity near the wall is overestimated. In addition, the channel height may vary in $z$-direction. Also the bubble protrusion angle can vary with channel depth. Since the value of $\vartheta$ is obtained using bright field images focused at the top of the channel, this partially clarifies the uncertainty in the interface curvature.

The way the effective slip length is calculated entails a small overestimation of the actual slip length that would have been obtained when using Eq. (2.2). The ratio of the effective slip calculated using Eq. (2.4) to that obtained using Eq. (2.2) varies with protrusion angle and surface porosity, but is for flat bubbles approximately 2.5 for $\varepsilon=0.38$ and 2 for $\varepsilon=0.54$ as simulations show. However, as also discussed in the appendix, we found that fitting a quadratic function to the full velocity profile leads to an underestimation of the slip length, in particular when the porosity is large [38]. The slippery wall makes the velocity profile non-parabolic, as the shear near the slippery surface is reduced. This is not described properly by a quadratic equation, which consequently leads to an overestimation of the shear rate at the wall. For that reason, we decided to 
determine the experimental slip length values by fitting a linear equation to the near-wall velocity components. Note, however, that both methods show the same qualitative behaviour for the dependency of the slip length on the protrusion angle and surface porosity.

The numerical profiles in Fig. 2.6 reveal an asymmetry in the effective slip length between positive and negative protrusion angles, which is in agreement with the literature $[16,17,19,21,22]$. Due to stability issues, we were unable to determine the flow field and calculate the slip lengths for negative or highly positive protrusion angles. The simulations also predict the slip length to be maximum at an slightly positive protrusion angle of $\vartheta=11^{\circ}$. The existence of an optimum protrusion angle was analytically and numerically predicted before $[17,21]$. When the protrusion angle exceeds the critical value of approximately $60^{\circ}$, the effective slip length becomes negative. The heterogeneously slippery but bumpy wall increases the flow resistance as compared to a smooth, non-slippery channel.

Besides increased roughness of the gas-solid wall, confinement effects also cause the slip length to decrease with increasing protrusion angle. For the microfluidic devices using in this study, the typical length scale of the channel is comparable with the bubble unit size, i.e. $L \approx H$, and therefore the change in effective height or cross-sectional area of the channel with protrusion angle is significant. The important role of channel confinement has been noted before in systems where the flow direction is parallel to the gas-liquid interfaces $[15,20]$.

\subsubsection{Comparison with analytical asymptotic solutions}

As a further verification of our experimental and numerical results, we compare these with some analytical asymptotic solutions for the effective slip length. These solutions were derived for Stokes flow in a circular pipe past periodically alternating and flat (i.e. $\vartheta=0^{\circ}$ ) shear-free and no-slip regions positioned transversely to the flow direction [10]. The following three distinct asymptotic limits (AL) for $b_{f}$ are considered:

$$
\begin{array}{rlrl}
b_{\mathrm{AL} 1} & \sim \frac{\varepsilon}{4} R & \varepsilon \rightarrow 0 \text { and } \delta \text { fixed } \\
b_{\mathrm{AL} 2} \sim \frac{\delta}{2 \pi} \ln \left[\sec \left(\varepsilon \frac{\pi}{2}\right)\right] R & & \delta \rightarrow 0 \text { and } \varepsilon \text { fixed } \\
b_{\mathrm{AL} 3} \sim \frac{\varepsilon}{4(1-\varepsilon)} R & \delta \rightarrow \infty \text { and } \varepsilon \text { fixed }
\end{array}
$$

Here, $R$ is a characteristic length scale. We define $R$ as the hydraulic radius, which is $R=H$ for two-dimensional flow between parallel plates. Since our system has only one-sided wall slip, half the channel height would also be a reasonable choice though. The channel confinement is expressed by $\delta=L / R_{h}=L / H$. The 
Table 2.1 - Comparison of the experimentally and numerically obtained effective slip lengths for $\vartheta \sim 0^{\circ}$ with the asymptotic limits given in Eq. (2.9). All slip length values are in $\mu \mathrm{m}$.

\begin{tabular}{ccccccc}
\hline$\varepsilon$ & $\delta$ & $b_{f}(\exp )$. & $b_{f}$ (num.) & $b_{\mathrm{AL} 1}$ & $b_{\mathrm{AL} 2}$ & $b_{\mathrm{AL} 3}$ \\
\hline 0.38 & 1.03 & $4.7 \pm 0.4$ & 4.6 & 4.6 & 1.5 & 7.5 \\
0.54 & 0.78 & $5.1 \pm 0.4$ & 4.8 & 6.9 & 2.6 & 15.1 \\
\hline
\end{tabular}

first asymptotic limit for the slip length corresponds to a channel with a given ratio of bubble unit length $L$ to channel height $H$ for which the bubble fraction approaches zero. The second limit is valid for a channel with a given surface porosity with bubbles that are infinitely small compared to the channel height. The third asymptote concerns a highly confined channel with a given surface porosity for which $L \gg H$.

In Table 2.1 we compare our experimentally and numerically obtained slip lengths with the asymptotic limits in Eq. (2.9). The numerical values are the slip lengths for $\vartheta=0^{\circ}$. The experimental values are the average slip lengths of the three data points closest to $\vartheta=0^{\circ}$, with an average protrusion angle of $\vartheta=5^{\circ} \pm 2^{\circ}$ for $\varepsilon=0.38$ and $\vartheta=4^{\circ} \pm 2^{\circ}$ for $\varepsilon=0.54$. For our bubble mattress, $0<\varepsilon<1$ and $\delta=\mathcal{O}(1)$. None of the asymptotic limits therefore applies. Notwithstanding the fact that for the bubble mattress $\varepsilon>0$, our slip length values are in good agreement with $b_{\mathrm{AL} 1}$, the first asymptotic limit. The asymptotic equations for $b_{\mathrm{AL} 2}$ and $b_{\mathrm{AL} 3}$ can be considered as the minimum $(\delta \rightarrow 0)$ and maximum $(\delta \rightarrow \infty)$ extremes for $b_{f}$, as for our $\delta=\mathcal{O}(1)$. Indeed, for all $\vartheta, b_{\mathrm{AL} 2}<b_{f}<b_{\mathrm{AL} 3}$. Our experimental and numerical results are therefore in accordance with the asymptotic limits presented in Eq. (2.9).

\subsubsection{Drag reduction}

The experimental and numerical results show that the gas-liquid interface curvature and the surface porosity have a strong effect on the amount of effective slip. Considering that we could actively control the protrusion angle of the gas-liquid menisci, this implies that it also would be possible to engineer and regulate the amount of wall slip or hydrodynamic drag in such heterogeneously slippery systems.

To highlight the capability of controlling the amount of wall slip in slippery microfluidic systems like our bubble mattress, we recalculated the experimental and numerical data in terms of the Fanning friction factor $f$. This friction factor is a direct measure of the pressure loss due to hydrodynamic drag. In Fig. 2.7, we present the values for the normalised effective friction factor $f / f_{0}$ as a function of the bubble protrusion angles $\vartheta$ for both $\varepsilon=0.38$ and $\varepsilon=0.54$. Since the Reynolds numbers differed ( $R e=8.9$ for $\varepsilon=0.38$ and $R e=10.5$ for $\varepsilon=0.54$ ), 


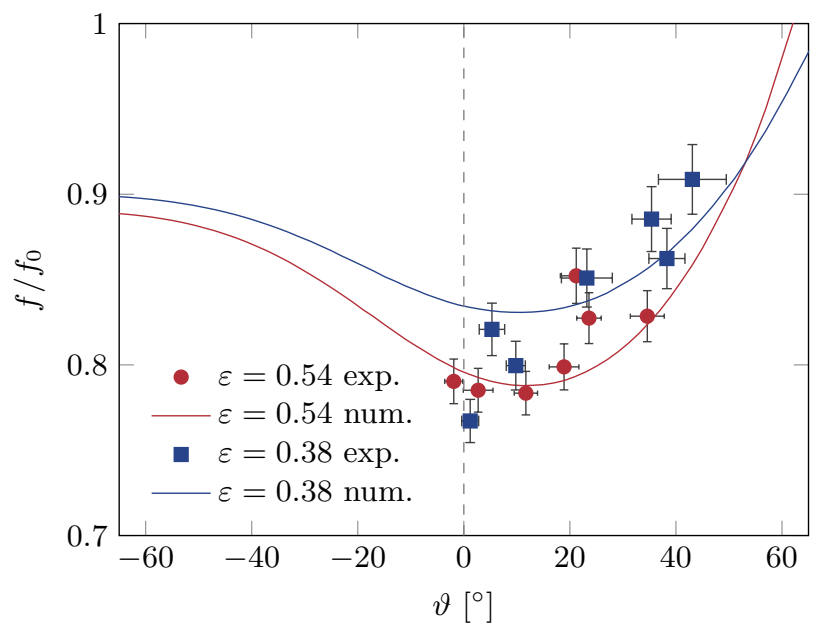

Figure 2.7 - Normalised friction factors $f / f_{0}$ as a function of the bubble protrusion angle $\vartheta$ for two different surface porosities $\varepsilon$, as obtained from experiments (symbols) and numerical simulations (lines).

the values for $f$ are normalised by its value for zero wall slip: $f_{0}=12 / R e$.

As Fig. 2.7 shows, the experimentally determined friction factors are smaller than one. This is expected, as for all protrusion angles $b_{f}>0$. The bubble mattress is therefore characterised by reduced friction when compared to slit flow with two no-slip walls. The numerical profiles show that when the protrusion angle is larger than the critical angle of approximately $60^{\circ}, f / f_{0}>1$. This indicates the transition point from hydrodynamic slippage to increased flow friction. The results presented in Fig. 2.7 also emphasise the considerable effect of the gas-liquid interface curvature on the amount of hydrodynamic slip.

The reduction in drag, compared with a non-slippery microchannel, equals $1-f / f_{0}$. Note that, according to Eq. (2.8), the maximum possible drag reduction is $75 \%$. This is achieved when $b_{f} / H \rightarrow \infty$. Our results indicate that we achieved, for a bubble mattress consisting of (relatively) flat bubbles with a protrusion angle of $0^{\circ}<\vartheta<15^{\circ}$, a drag reduction of $17 \%$ and $21 \%$ for $\varepsilon=0.38$ and $\varepsilon=0.54$, respectively.

\subsection{Conclusion}

In this chapter, we presented a microfluidic device in which a stable, slippery bubble mattress could be established that allowed active manipulation of the flow resistance. The design of the device made it possible to control the supply of gas, and thus to manipulate the protrusion angle of the micrometre-sized gas 
bubbles. The experimental measurements using micro-particle image velocimetry, performed for a wide range of protrusion angles, revealed a strong dependency of the effective slip length on the gas-liquid interface curvature. These measurements were validated by numerical simulations. Reappraisal of the results in terms of the effective friction factor emphasised that such microfluidic devices can be used to control hydrodynamic drag. This active control can be valuable for a further quantification of the impact of slippery surfaces on the transport phenomena near the interface and in the bulk [39, 40].

\section{References}

[1] N. Churaev, V. Sobolev, and A. Somov, Slippage of liquids over lyophobic solid surfaces, J. Colloid Interf. Sci. 97, 574 (1984).

[2] O. I. Vinogradova, Slippage of water over hydrophobic surfaces, Int. J. Miner. Process. 56, 31 (1999).

[3] D. C. Tretheway and C. D. Meinhart, Apparent fluid slip at hydrophobic microchannel walls, Phys. Fluids 14, L9 (2002).

[4] C.-H. Choi, K. J. A. Westin, and K. S. Breuer, Apparent slip flows in hydrophilic and hydrophobic microchannels, Phys. Fluids 15, 2897 (2003).

[5] P. Joseph and P. Tabeling, Direct measurement of the apparent slip length, Phys. Rev. E 71, 035303 (2005).

[6] C. Neto, D. R. Evans, E. Bonaccurso, H.-J. Butt, and V. S. J. Craig, Boundary slip in Newtonian liquids: a review of experimental studies, Rep. Prog. Phys. 68, 2859 (2005).

[7] E. Lauga, M. P. Brenner, and H. A. Stone, in Springer handbook of experimental fluid mechanics, edited by C. Tropea, A. L. Yarin, and J. F. Foss (Springer Berlin Heidelberg, 2007), pp. 1219-1240.

[8] J. P. Rothstein, Slip on superhydrophobic surfaces, Annu. Rev. Fluid Mech. 42, 89 (2010).

[9] K. Watanabe, Y. Udagawa, and H. Udagawa, Drag reduction of Newtonian fluid in a circular pipe with a highly water-repellent wall, J. Fluid Mech. 381, 225 (1999).

[10] E. Lauga and H. A. Stone, Effective slip in pressure-driven Stokes flow, J. Fluid Mech. 489, 55 (2003).

[11] J. Ou, B. Perot, and J. P. Rothstein, Laminar drag reduction in microchannels using ultrahydrophobic surfaces, Phys. Fluids 16, 4635 (2004).

[12] J. Ou and J. P. Rothstein, Direct velocity measurements of the flow past drag-reducing ultrahydrophobic surfaces, Phys. Fluids 17, 103606 (2005).

[13] C.-H. Choi and C.-J. Kim, Large slip of aqueous liquid flow over a nanoengineered superhydrophobic surface, Phys. Rev. Lett. 96, 1 (2006). 
[14] D. Byun, J. Kim, H. S. Ko, and H. C. Park, Direct measurement of slip flows in superhydrophobic microchannels with transverse grooves, Phys. Fluids 20, 113601 (2008).

[15] P. Tsai, A. M. Peters, C. Pirat, M. Wessling, R. G. H. Lammertink, and D. Lohse, Quantifying effective slip length over micropatterned hydrophobic surfaces, Phys. Fluids 21, 112002 (2009).

[16] J. Hyväluoma, C. Kunert, and J. Harting, Simulations of slip flow on nanobubble-laden surfaces., J. Phys. Cond. Matter 23, 184106 (2011).

[17] C.-O. Ng and C. Y. Wang, Effective slip for Stokes flow over a surface patterned with two- or three-dimensional protrusions, Fluid Dyn. Res. 43, 065504 (2011).

[18] S. Schmieschek, A. Belyaev, J. Harting, and O. Vinogradova, Tensorial slip of superhydrophobic channels, Phys. Rev. E 85, 016324 (2012).

[19] A. Steinberger, C. Cottin-Bizonne, P. Kleimann, and E. Charlaix, High friction on a bubble mattress., Nat. Mater. 6, 665 (2007).

[20] C. J. Teo and B. C. Khoo, Flow past superhydrophobic surfaces containing longitudinal grooves: effects of interface curvature, Microfluid. Nanofluid. 9, 499 (2010).

[21] A. M. J. Davis and E. Lauga, Geometric transition in friction for flow over a bubble mattress, Phys. Fluids 21, 011701 (2009).

[22] J. Hyväluoma and J. Harting, Slip flow over structured surfaces with entrapped microbubbles, Phys. Rev. Lett. 100, 246001 (2008).

[23] R. Maboudian, W. Ashurst, and C. Carraro, Self-assembled monolayers as anti-stiction coatings for MEMS: characteristics and recent developments, Sensor Actuat. A Phys. 82, 219 (2000).

[24] A. Stalder, G. Kulik, D. Sage, L. Barbieri, and P. Hoffmann, A snake-based approach to accurate determination of both contact points and contact angles, Colloid Surface A 286, 92 (2006).

[25] M. Abramoff, P. J. Magalhães, and S. J. Ram, Image processing with ImageJ, Biophotonics Intl 11, 36 (2004).

[26] C. L. M. H. Navier, Mémoire sur les lois du mouvement des fluids, Mem. Acad. Sci. Int. Fr. 6, 389 (1823).

[27] P. Joseph, C. Cottin-Bizonne, J.-M. Benoît, C. Ybert, C. Journet, P. Tabeling, and L. Bocquet, Slippage of water past superhydrophobic carbon nanotube forests in microchannels, Phys. Rev. Lett. 97, 1 (2006).

[28] D. Schäffel, K. Koynov, D. Vollmer, H.-J. Butt, and C. Schönecker, Local flow field and slip length of superhydrophobic surfaces, Phys. Rev. Lett. 116, 134501 (2016).

[29] S. C. Hendy and N. J. Lund, Effective slip boundary conditions for flows over nanoscale chemical heterogeneities, Phys. Rev. E 76, 066313 (2007).

[30] Y. A. Cengel, Heat transfer: a practical approach, 2nd ed. (McGraw-Hill, 
New York, 2002).

[31] R. B. Bird, W. E. Stewart, and E. N. Lightfoot, Transport phenomena, 2nd ed. (John Wiley \& Sons, New York, 2007).

[32] P. Gao and J. J. Feng, Enhanced slip on a patterned substrate due to depinning of contact line, Phys. Fluids 21, 102102 (2009).

[33] M. S. Bobji, S. V. Kumar, A. Asthana, and R. N. Govardhan, Underwater sustainability of the "Cassie" state of wetting, Langmuir 25, 12120 (2009).

[34] R. Poetes, K. Holtzmann, K. Franze, and U. Steiner, Metastable underwater superhydrophobicity, Phys. Rev. Lett. 105, 166104 (2010).

[35] M. A. Samaha, H. Vahedi Tafreshi, and M. Gad-el Hak, Sustainability of superhydrophobicity under pressure, Phys. Fluids 24, 112103 (2012).

[36] P. Huang, J. S. Guasto, and K. S. Breuer, The effects of hindered mobility and depletion of particles in near-wall shear flows and the implications for nanovelocimetry, J. Fluid Mech. 637, 241 (2009).

[37] C. J. Kähler, S. Scharnowski, and C. Cierpka, On the uncertainty of digital PIV and PTV near walls, Exp. Fluids 52, 1641 (2012).

[38] A. S. Haase, Transport over a bubble mattress, MSc thesis, University of Twente, 2012.

[39] L. Bocquet and J.-L. Barrat, Flow boundary conditions from nano- to microscales, Soft Matter 3, 685 (2007).

[40] L. Bocquet and E. Lauga, A smooth future?, Nat. Mater. 10, 334 (2011). 


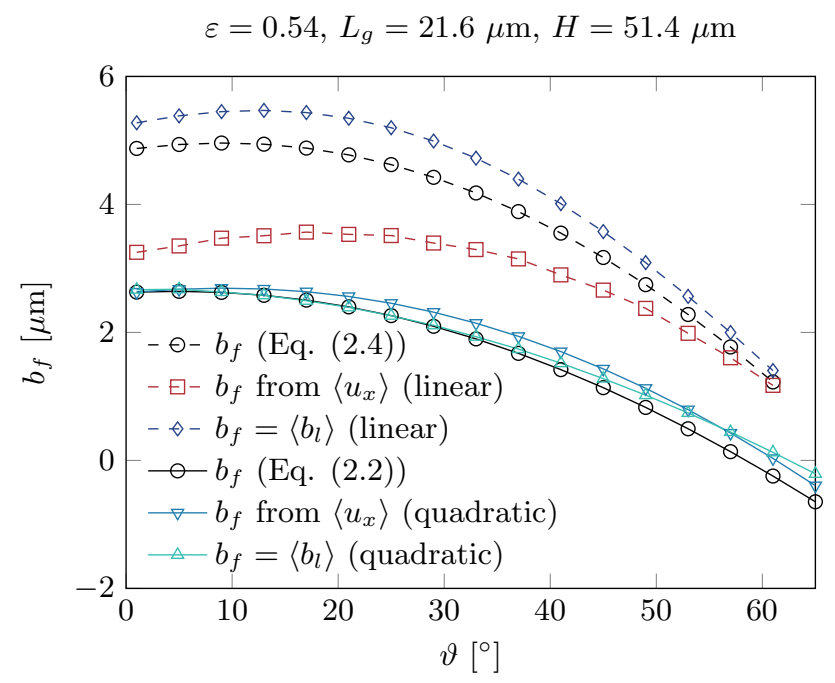

Figure 2.8 - A numerically calculated velocity field has been utilised to evaluate various methods for computing the effective slip length $b_{f}$, which can be calculated from the average velocity profile $\left\langle u_{x}\right\rangle$ or by averaging of the local slip lengths $b_{l}$. The shear rate at the wall can be obtained by fitting a linear equation to the velocity profile near the wall, or by calculation when fitting a quadratic equation to the full velocity profile.

\section{Appendix}

Various methods to compute the effective slip length $b_{f}$ have been evaluated based on a numerically calculated flow field for a bubble mattress with a surface porosity of $\varepsilon=0.54$ and a protrusion angle of $\vartheta>0^{\circ}$. The results are plotted in Fig. 2.8. The following observations can be made.

- There is a clear difference in the obtained effective slip length when using a linear fit to a part of the velocity profile and when fitting a quadratic equation to the full velocity profile. The linear approach clearly leads to an overestimation of the slip length, but the difference with the actual slip length obtained using Eq. (2.2) decreases with increasing protrusion angle. The closer to the wall the shear rate is determined, the more accurate the linear approach is. However, the maximum bubble protrusion depth determines the minimum $y$-position of the line at which the shear rate can be calculated.

- For the quadratic approach, it does not matter if the effective slip length is obtained from the average velocity profile, i.e. from $\left\langle u_{x}\right\rangle$, or by averaging of the local slip lengths, i.e. $b_{f}=\left\langle b_{l}\right\rangle$.

- This does matter, however, for the linear approach. Calculating the effective slip length by averaging of the local slip lengths gives a profile that is comparable with the one obtained using Eq. (2.4). Computing the effective slip length from 


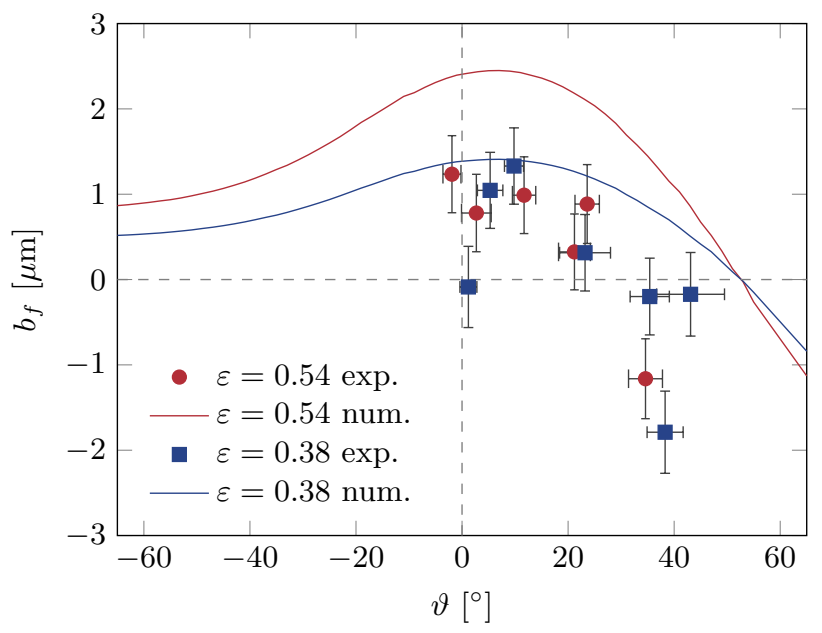

Figure 2.9 - Effective slip length $b_{f}$ as a function of the bubble protrusion angle $\vartheta$ for two different surface porosities $\varepsilon$, obtained by $\mu \mathrm{PIV}$ experiments (symbols) and numerical simulations (lines). The slip length is obtained by using the shear rate at the wall as calculated from the fitted quadratic equation to the local velocity profile. An outlier value $\left(b_{f}=3.7 \pm 0.5 \mu \mathrm{m}\right.$ for $\vartheta=19^{\circ} \pm 3^{\circ}$ for $\left.\varepsilon=0.54\right)$ has been omitted from the graph.

the average velocity profile $\left\langle u_{x}\right\rangle$ results in a significantly different slip length profile, with a maximum that is shifted towards higher protrusion angles.

Fig. 2.8 therefore shows that by fitting a quadratic equation, the most accurate results are obtained. If not possible, the linear approach should be followed. In that case, the effective slip length can best be calculated by averaging of the local slip lengths.

Fig. 2.9 illustrates that, when experimentally evaluating the effective slip length following the quadratic approach, the results are quantitatively very different from those calculated numerically. As discussed in Sec. 2.3.2, a quadratic equation does not always describe the profile in the vicinity of the wall properly. Additionally, velocity vectors near the wall obtained using $\mu$ PIV may be biased due to difficulties in measuring the velocity near the wall and other physical effects not accounted for when doing the $\mu \mathrm{PIV}$ measurements. When taking the results presented in Fig. 2.8 and Fig. 2.9 into consideration, we therefore decided to evaluate and present the effective slip lengths obtained by the linear approach, with the effective slip length being the average of the local slip lengths. 




\title{
CHAPTER 3
}

\section{Why bumpy is better: the role of the dissipation distribution in slip flow over a bubble mattress}

\begin{abstract}
It has been observed that the amount of effective slip for transverse flow over a bubble mattress is maximum for bubbles that protrude somewhat in the channel flow. In this paper we provide an explanation for this characteristic feature by analysing the spatial distribution of viscous dissipation for bubbles of varying protrusion angles. Bubbles protruding in the channel act as obstacles and reduce the effective channel height, thereby increasing the viscous dissipation in the bulk flow. At small scales, however, our numerical analysis reveals that increasing the bubble protrusion angle reduces the dissipation near the contact points of the no-slip channel wall and the no-shear bubble surface. We obtain an analytical expression to quantify this effect based on classical corner flow solutions. The two antagonistic effects, decreased dissipation near the bubble corners and increased dissipation on larger scale, explain why the effective effective slip length is maximum for a bubble mattress that is slightly bumpy.
\end{abstract}

This chapter has been published as

A.S. Haase, J.A. Wood, R.G.H. Lammertink and J.H. Snoeijer, Why bumpy is better: the role of the dissipation distribution in slip flow over a bubble mattress, Phys. Rev. Fluids 1, 054101 (2016). 


\subsection{Introduction}

Superhydrophobic surfaces are commonly used to optimise transport in microfluidic and nanofluidic systems [1-3]. The gas present in the micro-structures of the slippery and water-repellent surfaces reduces the overall friction between a flowing liquid and the wall, compared to flat non-slippery surfaces. The menisci of the gas bubbles are often assumed to be shear-free. This leads to small but finite effective slip velocities at the interface, which can enhance interfacial transport. The amount of wall slip is commonly expressed by the slip length $b$, as expressed by Navier's law [4]. At the surface, $\mathbf{u}-b \partial_{n} \mathbf{u}=\mathbf{0}$. Here, $\mathbf{u}$ is the liquid velocity and $\partial_{n}$ is the derivative normal to the surface. When considering slip over superhydrophobic surfaces, $b$ denotes the effective slip length experienced by the flow on scales larger than the bubbles.

For flat, hybrid gas-solid surfaces, the effective slip length is always positive. This may not be true for superhydrophobic surfaces containing curved gas-liquid interfaces. For transverse flow over a bubble mattress as shown in Fig. 3.1, which is a two-dimensional superhydrophobic surface consisting of an array of no-shear gas bubbles with no-slip walls in between, the amount of slip strongly depends on the curvature of the bubble interface (defined by the protrusion angle $\vartheta$, shown in Fig. 3.1). This dependence shows two characteristic features: slip is maximum for a small but finite non-zero protrusion angle $\vartheta$, but when the angle exceeds a critical value, the slip length becomes negative.

The first to demonstrate the existence of a critical protrusion angle for negative slip were Steinberger et al. [5]. They found that the slip length decreases when the protrusion angle $\vartheta$ increases. For strongly protruding bubbles, negative slip lengths were computed, implying that the flow is retarded by the superhydrophobic surface. Similarly, Legendre and Colin [6] showed that, compared to a non-slippery surface, wall drag always increases for linear shear flow over stationary, shear-free hemispherical bubbles.

The strong dependence of the effective slip length on the bubble protrusion angle $\vartheta$ was confirmed numerically by Hyväluoma and Harting [7]. They also found, considering infinitely long cylindrical bubbles, that the slip length strongly depends on the flow direction. When the flow is parallel to the bubble slots, the slip length $b$ is always positive [8-10]. The streamlines are straight and no roughness is encountered by the flow. In the case of transverse flow, however, the slip length is negative when the protrusion angle exceeds a critical value. The surface is rough due to the protruding bubbles, giving rise to negative slip lengths.

Davis and Lauga [11] derived an analytical expression for the effective slip length for transverse shear flow over a bubble mattress in the dilute limit, i.e. for a surface porosity $\varepsilon \ll 1$. Their two-dimensional model showed good qualitative agreement with previous numerical results [5, 7]. However, the theory by Davis 
and Lauga revealed a curious feature: the slip length is maximum for a small but non-zero optimum protrusion angle of $\vartheta=14^{\circ}$. For larger protrusion angles, the slip length decreases and becomes negative when $\vartheta$ exceeds the critical angle of $\vartheta=65^{\circ}$. Later studies confirmed the general validity of the model, even for a three-dimensional surface containing spherical bubbles [12-15].

Teo and Khoo [15] showed that the optimum and critical protrusion angles depend on the channel confinement, which is the ratio of channel height to projected bubble diameter. When increasing the confinement, the values of the optimal and critical protrusion angle decrease as the bubbles more readily obstruct the flow in shallow channels. The confinement effect becomes stronger for high surface porosities. They also showed some characteristics of the flow field along the channel wall and the bubble surface to explain the phenomenon of negative slip. Their numerical results indicated that with increasing protrusion angle, the wall shear stress near the contacts points of wall and bubble decreases, while the static pressure difference between the front and rear halves of the bubble interface increases. This latter effect dominates beyond the critical protrusion angle, resulting in negative slip lengths. However, even though the existence of an optimal protrusion angle is very robust and consistently observed in theory and simulations, it remains an open question why for transverse flow over a bubble mattress the amount of slip is maximum for a finite, non-zero protrusion angle.

In this paper we will provide an explanation why the effective slip length over a bubble mattress exhibits a maximum for a certain angle. To formulate an answer, we establish an explicit connection between effective slip and viscous dissipation, and consider the spatial distribution of viscous dissipation. As the numerical and analytical results reveal, there are two opposing effects acting at different scales that result in an optimal angle for which the overall dissipation is minimum, or the effective slip is maximum.

\subsection{Mathematical formulation}

\subsubsection{Governing equations}

Fig. 3.1 depicts the geometry of the system considered in this study. The periodic model with length $L$ is two-dimensional, and the undeformable, spherical bubbles are considered to be infinitely long. The top wall is non-slippery, while the bottom wall is a no-slip surface containing a no-shear bubble of width $L_{g}$ and having a radius of curvature of $R=L_{g} /(2 \sin \vartheta)$. The protrusion angle $\vartheta$ of the bubble was varied. The ratio of channel height to domain length was set to $a=H / L=2$. The porosity was fixed at $\varepsilon=L_{g} / L=1 / 2$. Parametric studies on the relationship between geometry and effective slip have been published before [11, 14, 15]. Here we aim to identify the physical mechanism that leads to the existence of an 


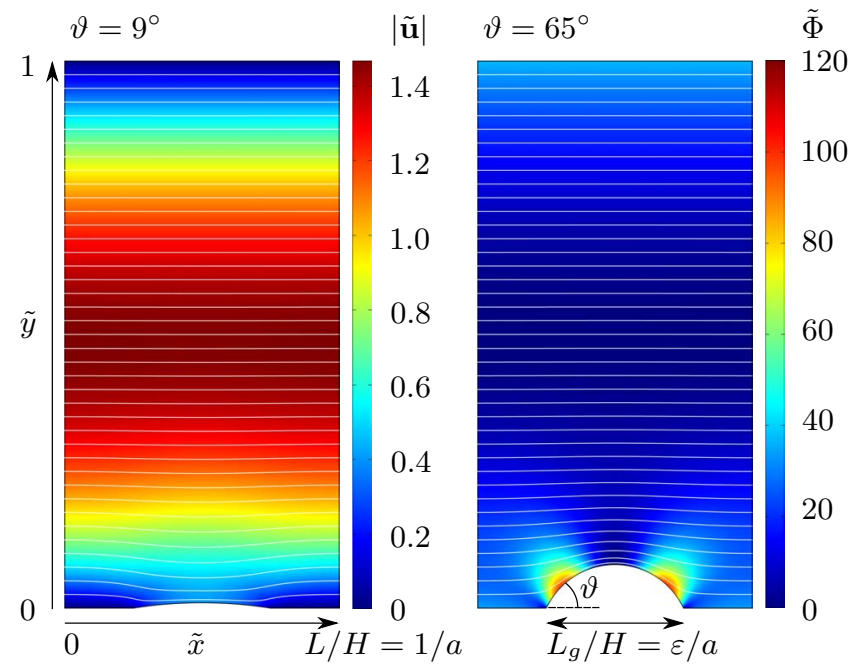

Figure 3.1 - Geometry of a viscous flow over a bubble mattress. A no-shear bubble with a protrusion angle $\vartheta$ is sticking out into the channel flow at the lower boundary. The boundary conditions in the horizontal direction are periodic. In the left figure, the colour map represents the velocity magnitude $|\tilde{\mathbf{u}}|$, while in the right figure the colour map shows the local viscous dissipation rate $\tilde{\Phi}$. The white lines are streamlines of the velocity field $\tilde{\mathbf{u}}$. The relevant dimensions are the channel height $H$, the width of periodicity $L$ and the bubble size $L_{g}$. Throughout the paper, all length scales are non-dimensionalised by the channel height $H$.

optimal protrusion angle for which slip is maximum.

Assuming that the Reynolds number $R e=u_{a v} \rho D / \mu$ is very small and that the flow is incompressible, momentum transport is described by the continuity and Stokes equations,

$$
\begin{gathered}
\tilde{\nabla} \cdot \tilde{\mathbf{u}}=0, \\
0=-\tilde{\nabla} \tilde{p}+\tilde{\nabla}^{2} \tilde{\mathbf{u}} .
\end{gathered}
$$

Here, $\tilde{\nabla}=\left(\partial_{x}, \partial_{y}\right), \tilde{\mathbf{u}}=\left(\tilde{u}_{x}, \tilde{u}_{y}\right)$ is the velocity vector, and $\tilde{p}$ is the pressure. The equations were made dimensionless using the height-averaged velocity $u_{a v}$, the channel height $H$, and the dynamic viscosity $\mu$. The scaled variables thus read $\tilde{\mathbf{x}}=\mathbf{x} / H, \tilde{\mathbf{u}}=\mathbf{u} / u_{a v}$ and $\tilde{p}=p H / \mu u_{a v}$.

The no-slip boundary condition is applied to the solid walls at the top and the bottom,

$$
\tilde{\mathbf{u}}=\mathbf{0} \text {. }
$$


The bubble surface is non-penetrable and shear-free, which is described by

$$
\left\{\begin{array}{l}
\tilde{\mathbf{u}} \cdot \tilde{\mathbf{n}}=0, \\
{\left[-\tilde{p} \mathbf{I}+\left(\nabla \tilde{\mathbf{u}}+(\nabla \tilde{\mathbf{u}})^{\mathrm{T}}\right)\right] \cdot \tilde{\mathbf{n}}=\mathbf{0} .}
\end{array}\right.
$$

The domain is periodic in the $\tilde{x}$-direction, i.e.

$$
\left\{\begin{array}{l}
\tilde{\mathbf{u}}(0, \tilde{y})=\tilde{\mathbf{u}}(1 / a, \tilde{y}), \\
\tilde{p}(0, \tilde{y})=\tilde{p}(1 / a, \tilde{y}) .
\end{array}\right.
$$

The equations were solved numerically using COMSOL Multiphysics 5.0. The standard relative tolerance was $1 \times 10^{-3}$. Here P2 + P1 discretisation (secondorder elements for velocity and first-order elements for pressure) was used to solve the Stokes equations. The mesh was refined near the walls by using a smaller mesh size and by using boundary layers, until the effective slip length became mesh-independent. The complete mesh consists of approximately 15500 domain elements and about 550 boundary elements. The numerical solution is obtained by imposing a pressure gradient. Owing to the linearity of the Stokes flow, the solution is subsequently scaled to achieve the desired average velocity of $\tilde{u}_{a v}=1$.

\subsubsection{Relating the effective slip length to viscous dissipation}

The reduction in flow resistance for a superhydrophobic wall is commonly quantified by an effective slip length. The effective slip length for a heterogeneously slippery surface is defined as the equivalent slip length required to achieve the same average velocity and pressure gradient past a homogeneous slip wall. Here, we will briefly repeat the relevant definitions and establish a formal link between the slip length and the total viscous dissipation in the fluid domain. The latter will be used to explain the non-monotonic dependence of the slip length on $\vartheta$.

Effective slip length. For a plane channel with a one-sided homogeneous slip wall, the pressure-driven velocity field reads

$$
\tilde{u}_{x}=\frac{1}{2} \frac{\partial \tilde{p}}{\partial \tilde{x}}\left[\left(\tilde{y}^{2}-\tilde{y}\right)-\frac{\tilde{b}}{1+\tilde{b}}(1-\tilde{y})\right],
$$

where $\tilde{b}=b / H$ is the scaled slip length. One verifies the no-slip condition $\tilde{u}_{x}=0$ at the upper boundary $\tilde{y}=1$, while at the lower boundary $\tilde{y}=0$ the solution satisfies Navier's slip condition $\tilde{u}_{x}=\tilde{b} \partial_{y} \tilde{u}_{x}$. The corresponding average liquid velocity is

$$
\tilde{u}_{a v}=-\frac{1}{12} \frac{\partial \tilde{p}}{\partial \tilde{x}}\left(\frac{1+4 \tilde{b}}{1+\tilde{b}}\right)
$$


This can be rewritten as an explicit expression for the effective slip length $\tilde{b}$,

$$
\tilde{b}=\left(-\tilde{u}_{a v}-\frac{1}{12} \frac{\partial \tilde{p}}{\partial \tilde{x}}\right) /\left(\tilde{u}_{a v}+\frac{1}{3} \frac{\partial \tilde{p}}{\partial \tilde{x}}\right),
$$

which was used to compute the slip lengths in our simulations of flow over a bubble mattress. For clarity we keep explicitly $\tilde{u}_{a v}$ in the various formulae, even though our dimensionalisation implies $\tilde{u}_{a v}=1$. Commonly in the literature, the slip length is non-dimensionalised based on $L_{g}$, the width of the bubble. In the present notation this reads

$$
\frac{2 b}{L_{g}}=\frac{2 a}{\varepsilon} \tilde{b} .
$$

Viscous dissipation. To explain why slip is optimal for an angle larger than zero degrees, it is useful to express slip in terms of the total viscous dissipation. The dimensionless viscous dissipation rate (per unit volume), in the case of incompressible flow, is given by $[16,17]$

$$
\tilde{\Phi}=2\left[\left(\frac{\partial \tilde{u}_{x}}{\partial \tilde{x}}\right)^{2}+\left(\frac{\partial \tilde{u}_{y}}{\partial \tilde{y}}\right)^{2}\right]+\left(\frac{\partial \tilde{u}_{y}}{\partial \tilde{x}}+\frac{\partial \tilde{u}_{x}}{\partial \tilde{y}}\right)^{2}
$$

where $\tilde{\Phi}=\Phi H^{2} / \mu u_{a v}^{2}$. The total dissipation in the two-dimensional domain is obtained by integrating $\tilde{\Phi}$ over the domain area $A$,

$$
\tilde{\Phi}_{A}=\iint_{A} \tilde{\Phi} \mathrm{d} A
$$

where we use the convention that the subscript $A$ implies an area integral of $\tilde{\Phi}$. Similarly, the dissipation in a streamtube $\tilde{\Phi}_{A \text {,str }}$ is obtained by integrating $\tilde{\Phi}$ over the area between two streamlines $A_{\text {str }}$. Using Gauss's theorem [16], one can derive that

$$
\iint_{A} \tilde{\Phi} \mathrm{d} A=-\tilde{u}_{x} \Delta \tilde{p} \int_{0}^{1} \mathrm{~d} \tilde{y}=-\frac{\tilde{u}_{a v}}{a} \frac{\partial \tilde{p}}{\partial \tilde{x}} .
$$

By combination of Eqs. (3.11) and (3.12) and subsequent elimination of the pressure gradient using Eq. (3.7), the total dissipation in the domain can be expressed as

$$
\tilde{\Phi}_{A}=\frac{12 \tilde{u}_{a v}^{2}}{a}\left(\frac{1+\tilde{b}}{1+4 \tilde{b}}\right) .
$$

Importantly, this equation gives a direct, monotonic relation between the effective slip length $\tilde{b}$ and the total viscous dissipation $\tilde{\Phi}_{A}$. As expected, a smaller dissipation implies a larger effective slip. Explaining why there exists a maximum in wall slip for a non-zero protrusion angle $\vartheta$ is therefore equivalent to understanding why there is a minimum in viscous dissipation at the same angle. 
(a) $a=2, \varepsilon=1 / 2$

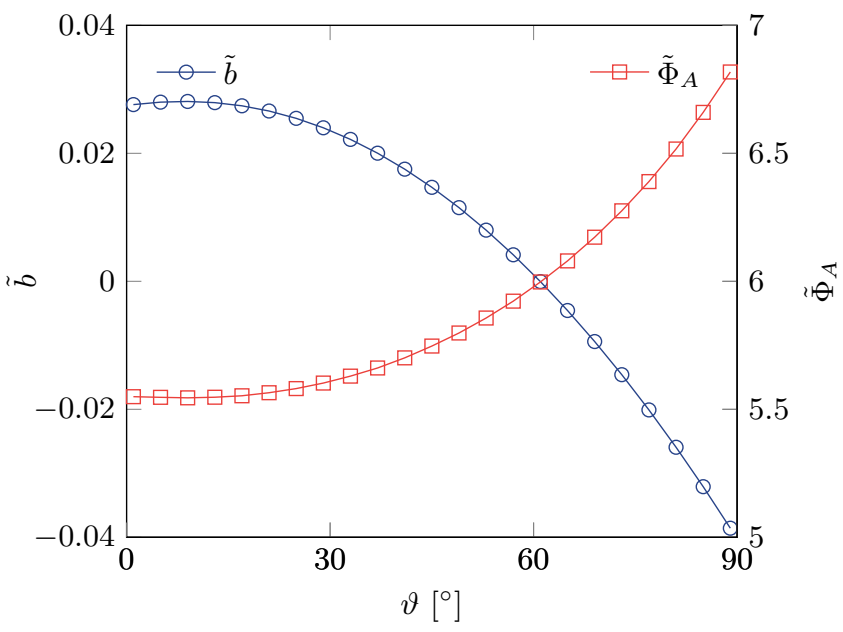

(b) $\varepsilon=1 / 2$

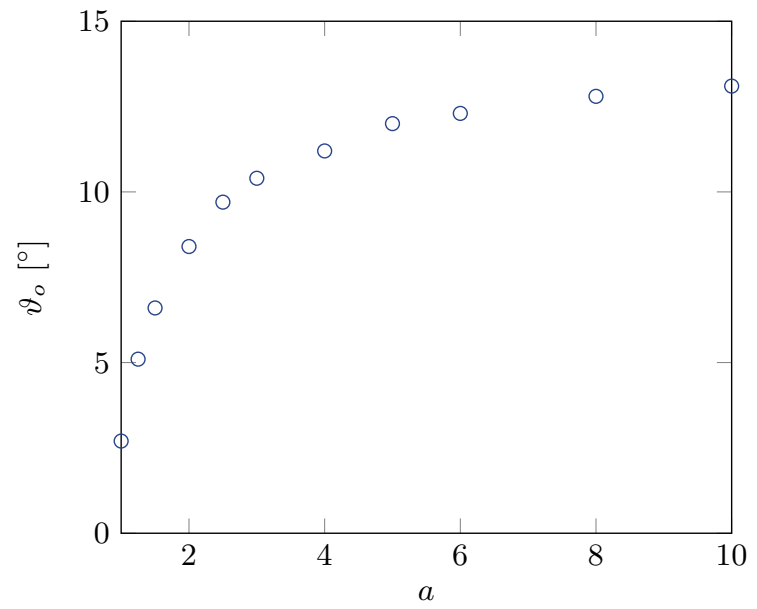

Figure 3.2 - (a) Dimensionless slip length $\tilde{b}$ and total dissipation $\tilde{\Phi}_{A}$ plotted a as function of the bubble protrusion angle $\vartheta$. The effective slip length and the total dissipation are related to each other by Eq. (3.13). When $\vartheta=9^{\circ}, \tilde{b}$ is maximum and $\tilde{\Phi}_{A}$ is minimum. (b) Dependence of the optimal protrusion angle $\vartheta_{o}$ on the confinement factor $a=H / L$.

\subsection{Results and discussion}

We now show the results of our numerical simulations, based on which we propose an explanation for the existence of an optimal protrusion angle. Fig. 3.2( $a$ ) displays the dimensionless slip length $\tilde{b}$ (blue circles), which is equivalent to 
$(\varepsilon / 2 a)\left(2 b / L_{g}\right)$ according to Eq. (3.9), as a function of the protrusion angle $\vartheta$. It reveals that slip is maximum for a finite positive protrusion angle of $\vartheta=9^{\circ}$ in this case. The same figure shows the corresponding total dissipation $\tilde{\Phi}_{A}$ (red squares) by the flow, which is related to the effective slip length according to Eq. (3.13). Maximum wall slip can indeed be expressed as minimum dissipation, since for the optimal protrusion angle $\vartheta_{o}$ of $9^{\circ}$ the total dissipation is minimum.

The protrusion angle for which the effective slip length is maximum, or the overall dissipation is minimum, depends on the confinement of the channel and the coverage density of the surface by bubbles. Fig. 3.2(b) illustrates that when the relative channel height is large, i.e. $a \gg 1$, the optimum protrusion angle converges to a value of $14^{\circ}$ for $\varepsilon=1 / 2$. This is similar to the optimum angle found by Davis and Lauga [11], although their expression is derived for shear flow over a bubble mattress in the dilute limit $(\varepsilon \ll 1)$. The existence of an optimum protrusion angle is therefore very robust, although the precise value depends on the geometry of the system.

To explain why the slip length is maximum for slightly protruding bubbles, we now consider the distribution of the viscous dissipation rate $\tilde{\Phi}$ in the fluid domain. These distributions are shown in Fig. 3.3( $a$ ) for several protrusion angles. The figures clearly show a gradual change of the spatial distribution of the viscous dissipation rate when increasing $\vartheta$. For small angles the dissipation rate is strongest near the bubble corners, where the boundary condition changes discontinuously from no-slip at the solid wall to no-shear at the bubble surface. The fluid flow close to the surface is significant for small angles, giving rise to large velocity gradients and hence high dissipation rates near the discontinuities. When the protrusion angle increases, we observe that the location of maximum $\tilde{\Phi}$ gradually shifts upward along the bubble surface. Similar trends were observed by Theo and Khoo [15], who considered the shear stress and static pressure at the lower wall.

In order to compare the dissipation distribution for different protrusion angles more quantitatively, we consider the dissipation in a streamtube $\tilde{\Phi}_{\mathrm{A} \text {,str }}$ versus the midpoint $\tilde{y}_{0}$ of the streamtube at $\tilde{x}=0$. This is shown in Fig. 3.3 $(b)$, with a more detailed zoom given in Fig. 3.3(c). The dissipation profiles depend non-monotonically on the height $\tilde{y}$, with a suppressed dissipation in the first few streamtubes $\left(\tilde{y}_{0}<0.05\right)$, which are passing close to the corners of the bubble. The dissipation near the corner decreases considerably with increasing protrusion angle. This indicates that the fluid between the bubbles becomes nearly stagnant, supporting the notion by Steinberger et al. [5] of an immobilised liquid layer close to the wall for large protrusion angles.

On the other hand, farther away from the solid wall $\left(\tilde{y}_{0}>0.05\right)$ the dissipation increases with $\vartheta$. The location of maximum dissipation shifts from the contact points towards the front and rear edge of the bubble surface. First, the pressure 


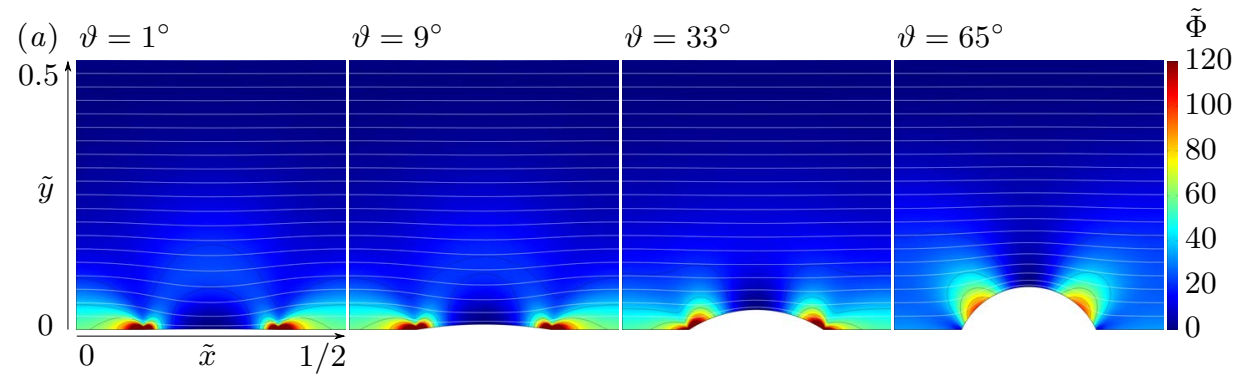

(b) $a=2, \varepsilon=1 / 2$

(c) magnification of $(b)$
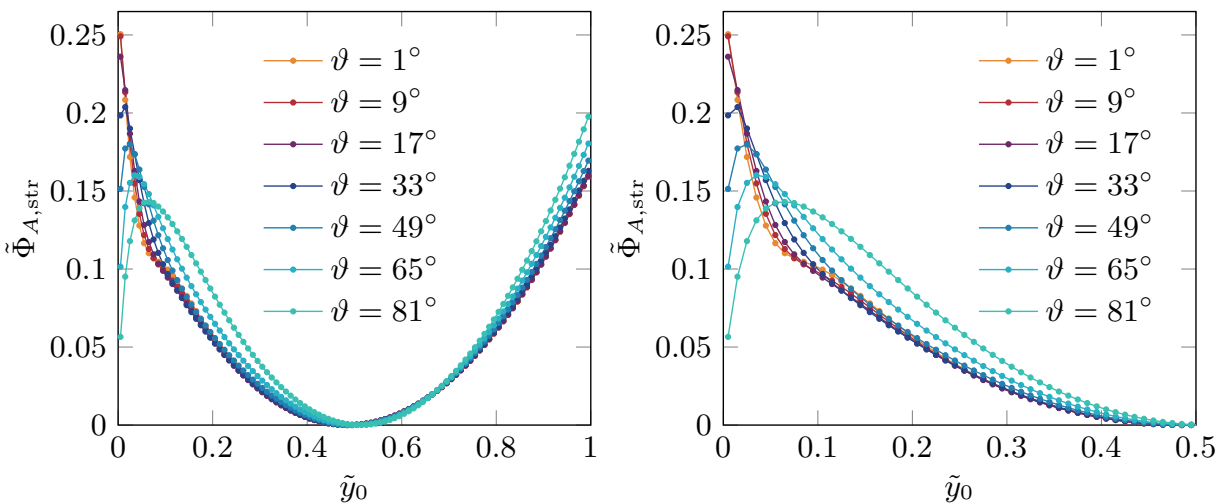

Figure 3.3 - The colour maps in (a) show the distribution of the dissipation rate $\tilde{\Phi}$ for four different protrusion angles for $0 \leq \tilde{y} \leq 0.5$. The streamlines of the velocity field $\tilde{\mathbf{u}}$ are shown in white. The black lines are contour lines of the dissipation rate $\tilde{\Phi}$. In $(b)$ and $(c)$, the viscous dissipation $\tilde{\Phi}_{A \text {,str }}$ in a streamtube of width $\Delta \tilde{y}=0.01$ is plotted against the midpoint of the streamtube $\tilde{y}_{0}$ at $\tilde{x}=0$.

difference over the bubble surface becomes larger when the protrusion angle increases [15]. Second, bubbles protruding in the channel increasingly form obstacles to the flow, acting as roughness elements [7]. These elements causing bending of the streamlines, which necessarily means that viscous dissipation increases. We also observe that for $\vartheta>33^{\circ}$ the dissipation increases near the upper wall. The strongly protruding bubbles reduce the effective channel height, leading to increased shear and higher dissipation rates in the bulk channel flow.

Fig. 3.3 is key to understanding why there exists an optimum protrusion angle for which effective slip is maximum, or viscous dissipation is minimum. Since $\tilde{\Phi}_{A}=\int \tilde{\Phi}_{A, \text { str }} \mathrm{d} \tilde{y}$, this figure reveals that there are two opposing trends. Near the surface, in the corners between bubble and wall, the dissipation decreases with increasing $\vartheta$, while globally the dissipation becomes larger with $\vartheta$. These two competing effects lead to a minimum in total dissipation, and therefore maximum 
slip, at an optimum protrusion angle of, in this case, $\vartheta_{o}=9^{\circ}$.

We now explain in detail why viscous dissipation is suppressed near the bubble corner when the protrusion angle increases. This analysis is based on the analytical corner flow solutions by Moffatt [18], who solved the flow field of a viscous fluid near a sharp corner between a rigid boundary and a free surface. It turns out that viscous corner flows exhibit a universal, self-similar structure that depends only on the boundary conditions (which are no-slip and no-shear in the present case) and on the corner angle. This allows us to compute the dissipation in the vicinity of the corner as a function of the protrusion angle $\vartheta$. In terms of the streamfunction $\psi$, the Moffatt corner flow solutions in polar coordinates $(\tilde{r}, \phi)$ are of the form [18]

$$
\psi(\tilde{r}, \phi)=A f(\phi) \tilde{r}^{\lambda} .
$$

For given angle $\vartheta$, the function $f(\phi)$ has a prescribed functional form (see the appendix to this chapter). Likewise, the exponent $\lambda$, which does not depend on $\phi$, can be determined from an eigenvalue problem that amounts to solving

$$
\sin 2 \alpha(\lambda-1)=(\lambda-1) \sin 2 \alpha
$$

where $\alpha=\pi-\vartheta$. The values of $\lambda$ (solid line) are plotted as a function of $\vartheta$ in Fig. 3.4( $a$ ), showing that $\lambda$ increases monotonically with the protrusion angle $\vartheta$. The amplitude $A$ in Eq. (3.14) cannot be determined from a local analysis of the corner flow, but depends on how the flow is driven far away from the corner.

We can now use the Moffatt solution to compute the dissipation rate near the corner. From Eq. (3.14), one can derive that

$$
\tilde{\Phi}=A^{2} g(\phi) \tilde{r}^{2 \lambda-4}
$$

which gives the distribution of the dissipation rate. The appendix to this chapter can be referred to for the full derivation. The resulting dissipation field is again self-similar. An example of the dissipation field is given in Fig. 3.4 $(d)$ for the case where $\vartheta=12^{\circ}$. While $g(\phi)$ and $\lambda$ are readily calculated and we therefore precisely know how they change with the protrusion angle $\vartheta$, this is not the case for $A$ : the amplitude of the corner flow depends on the large-scale characteristics of the flow, which requires a fully resolved flow field. For this purpose we numerically determined the amplitude $A$ from the explicit form of the stream function by Davis and Lauga [11] for shear flow over a bubble mattress in the dilute limit (which is given in the Appendix for completeness). Obviously, the solution by Davis and Lauga should implicitly contain the universal flow structure expressed by Eq. (3.14) at small distances from the corner. This has been verified by fitting the Moffatt solution to the solution of Davis and Lauga, giving us $\lambda(\vartheta)$. The resulting values (circles) are superimposed on the analytical values (solid line) in 
(a) $\lambda$ as function of $\vartheta$

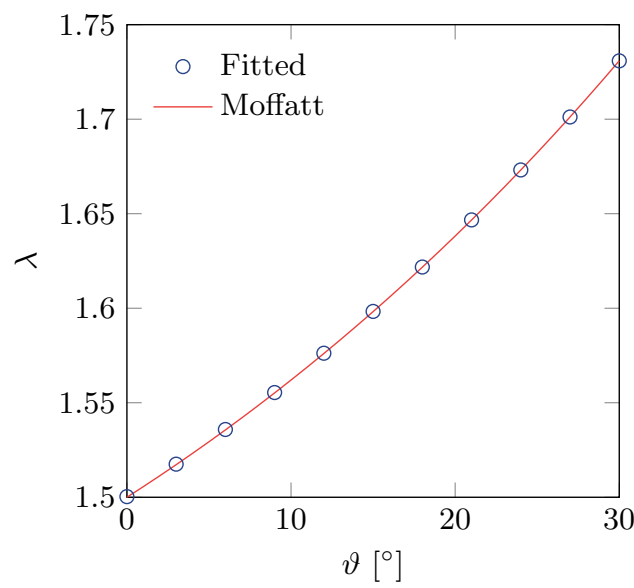

(c) $\tilde{\Phi}_{\max }(\vartheta) / \tilde{\Phi}_{\max }(0)$ at $\tilde{r}=10^{-5}$

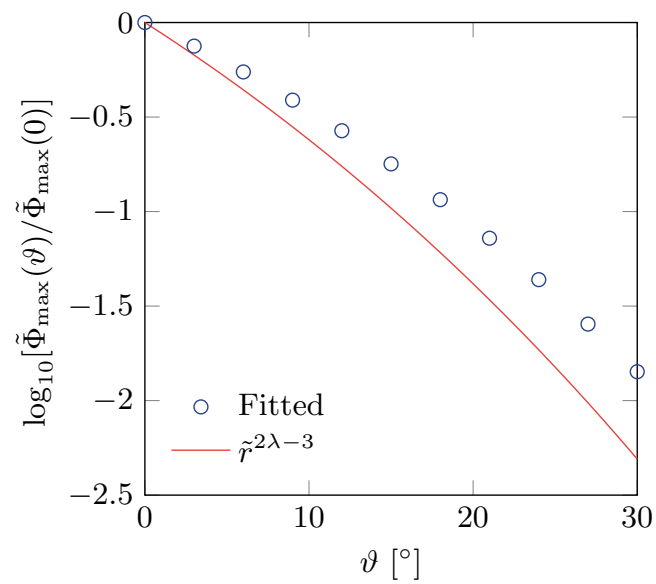

(b) $A f(\phi)$ for various $\vartheta$



(d) $\tilde{\Phi}$ for $\vartheta=12^{\circ} \quad \log _{10}(\tilde{\Phi})$

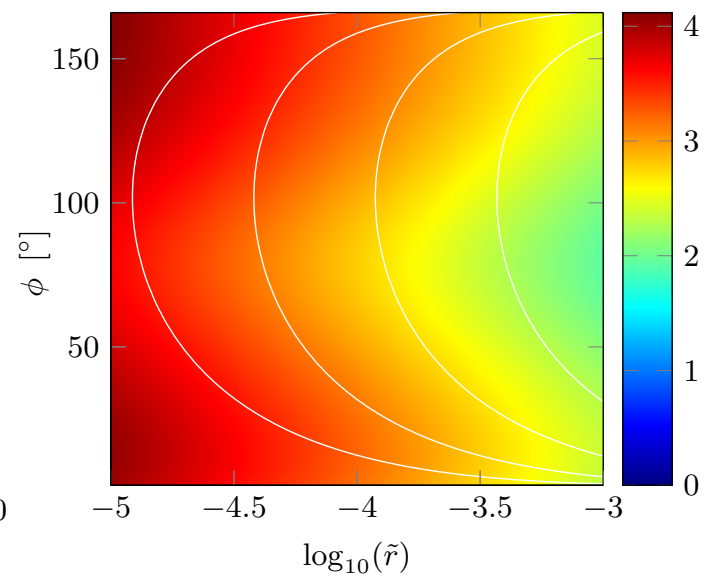

Figure 3.4 - To prove that the viscous dissipation in the vicinity of the bubble corners decreases with increasing protrusion angle $\vartheta$, the flow near the corner is analysed using the Moffatt corner flow solution $\psi=A f(\phi) r^{\lambda}[18]$. (a) Exponent $\lambda$ plotted as a function of $\vartheta$, obtained from fitting the Moffatt solution to the analytical solution of Davis and Lauga [11] (circles) and by solving Eq. (3.15) (solid line). (b) Plot of $A f(\phi)$ for various $\vartheta$. Both the amplitude $A$ and the function $f(\phi)$ are approximately constant. (c) Maximum dissipation rate $\tilde{\Phi}_{\max }(\vartheta)$, determined at $\tilde{r}=10^{-5}$ and normalised by $\tilde{\Phi}_{\max }(0)$. Based on a theoretical analysis, it is predicted that near the corner $\tilde{\Phi}(\vartheta) / \tilde{\Phi}(0) \sim \tilde{r}^{2 \lambda-3}$ (Eq. $(3.17))$. (d) Dissipation rate $\tilde{\Phi}$ for a protrusion angle of $\vartheta=12^{\circ}$, with some streamlines of $\tilde{\mathbf{u}}$ plotted in white. The bubble surface is located at $\left(\tilde{r}, 168^{\circ}\right)$. 
Fig. 3.4(a). Subsequently, the product $A f(\phi)$ is plotted in Fig. 3.4(b) for various protrusion angles. Most importantly, it reveals that changes in the function $f(\phi)$ with $\vartheta$ are only minor and that the amplitude $A$ is approximately constant for the range of $\vartheta$ considered.

From the above considerations we infer that the dominant dependence of the dissipation rate $\tilde{\Phi}$ on the protrusion angle is encoded in $\lambda(\vartheta)$, and in particular in the dependence $\tilde{\Phi} \propto \tilde{r}^{2 \lambda-4}$. To compare the viscous dissipation rate $\tilde{\Phi}$ at different protrusion angles $\vartheta$, it is convenient to normalise $\tilde{\Phi}$ by the value of $\tilde{\Phi}$ for $\vartheta=0^{\circ}$. Since $\lambda=3 / 2$ when $\vartheta=0^{\circ}$, we obtain

$$
\tilde{\Phi}(\vartheta) / \tilde{\Phi}(0) \sim \tilde{r}^{2 \lambda-3}
$$

which provides an analytical estimate of how the rate of dissipation depends on $\vartheta$. The result is plotted as a solid line in Fig. 3.4(c) for $\tilde{r}=10^{-5}$. The selected value of $\tilde{r}$ is arbitrary: as long as $\tilde{r} \ll 1$ the flow field is self-similar and graphs of Eq. (3.17) will exhibit the same trend. Fig. 3.4(c) confirms that, as expected, in the direct vicinity of the corner the dissipation rate $\tilde{\Phi}$ strongly decreases with increasing protrusion angle. The numerically determined normalised dissipation rates (circles), obtained from the computed flow and dissipation fields utilising the Moffatt solution using the previously determined values of $A(\vartheta)$, are also plotted in Fig. 3.4(c). It shows that indeed the numerical values are well approximated by Eq. (3.17). The small differences between the calculated values and the analytical estimation can be attributed to the minor changes in the product $A^{2} g(\phi)$ (and in particular to $g(\phi)$, since $A$ is nearly constant) with protrusion angle $\vartheta$. The corner analysis therefore explains that the suppressed dissipation near the bubble corners with increasing protrusion angle is mainly due to the increase of the exponent $\lambda$ with the protrusion angle.

Finally, the dissipation distribution is not only valuable in explaining the existence of an optimal protrusion angle, it can also be used to predict the critical protrusion angle $\vartheta_{c}$ for which the slip length becomes negative. A negative slip length indicates that the friction for flow over a slippery surface is larger than for Hagen-Poiseuille flow through a smooth, non-slippery channel. For HagenPoiseuille flow we find that $\tilde{\Phi}_{\mathrm{HP}}=36\left(4 \tilde{y}^{2}-4 \tilde{y}+1\right)$. This gives for a confinement factor of $a=2$ an overall dissipation of $\tilde{\Phi}_{A}=6$. This matches with Fig. 3.2, as $\tilde{b}=0$ when $\tilde{\Phi}_{A}=6$ for $\vartheta=61^{\circ}$.

To identify the critical protrusion angle, we consider the difference in local dissipation rate $\Delta \tilde{\Phi}=\tilde{\Phi}-\tilde{\Phi}_{\mathrm{HP}}$. Fig. 3.5 shows $\Delta \tilde{\Phi}$ for four different protrusion angles. These plots again illustrate the fact that with increasing protrusion angle, the dissipation decreases near the corners of the bubbles and increases in the bulk channel flow. When increasing $\vartheta$, for an increasing fraction of the channel $\Delta \tilde{\Phi}$ is positive (and therefore changes colour from blue to green). Since 




Figure 3.5 - For four different protrusion angles $\vartheta$ the difference in dissipation rate $\Delta \tilde{\Phi}=\tilde{\Phi}-\tilde{\Phi}_{\mathrm{HP}}$ is plotted. Each contour level corresponds to $\Delta \tilde{\Phi}=25$. Here $\Delta \tilde{\Phi}<0$ for the blue contour levels, and $\Delta \tilde{\Phi}>0$ for the green and red contour levels. When $\vartheta$ is larger than the critical protrusion angle of, in this case, $61^{\circ}$, in the channel mainly $\Delta \tilde{\Phi}>0$, indicating increased friction with respect to Hagen-Poseuille flow through a non-slippery channel.

for large protrusion angles the dissipation in the bulk flow is dominant over the dissipation near the bubble corners, the slip length should become negative when the dissipation in the main channel becomes larger than for Hagen-Poiseuille flow. Thus, when $\vartheta \geq \vartheta_{c}=61^{\circ}$ we expect that in the main channel $\Delta \tilde{\Phi}>0$. Fig. 3.5 confirms this expectation, as in the channel $\Delta \tilde{\Phi}$ is mainly negative (coloured blue) when $\vartheta=57^{\circ}<61^{\circ}$ and predominantly positive (coloured green) when $\vartheta \geq 61^{\circ}$.

\subsection{Conclusion}

The effective slip length for transverse flow over a bubble mattress is largest for bubbles that slightly protrude in the channel flow, and not for completely flat bubbles. To explain the existence of this optimal protrusion angle, we established an explicit connection between the slip length and the viscous dissipation rate and subsequently analysed the spatial dissipation distribution. The total dissipation in the domain can be directly related to the effective slip length.

The numerical results reveal that near the contact points of the no-slip wall and the no-shear bubble surface, the viscous dissipation rate is maximum when the bubbles are flat. The different boundary conditions of the wall and bubble give rise to large velocity gradients, leading to high dissipation rates. When increasing 
the bubble protrusion angle, the dissipation close to these discontinuities decreases. An analytical treatment of the dissipation rate in the corner utilising a classical corner flow solution provides proof of this observation. On the other hand, bubbles sticking out into the channel flow act as obstacles and reduce the effective channel height, thereby increasing the dissipation in the bulk flow.

We conclude that increasing the protrusion angle of a bubble has two opposing effects: it reduces dissipation in the vicinity of the bubble corners, while it increases viscous dissipation in the bulk of the channel. This explains the existence of an optimum protrusion angle larger than zero degrees for which the total dissipation in the channel is minimum or the amount of effective slip is maximum.

\section{References}

[1] C. Neto, D. R. Evans, E. Bonaccurso, H.-J. Butt, and V. S. J. Craig, Boundary slip in Newtonian liquids: a review of experimental studies, Rep. Prog. Phys. 68, 2859 (2005).

[2] J. P. Rothstein, Slip on superhydrophobic surfaces, Annu. Rev. Fluid Mech. 42, 89 (2010).

[3] T. Lee, E. Charrault, and C. Neto, Interfacial slip on rough, patterned and soft surfaces: a review of experiments and simulations, Adv. Colloid Interfac. 210, 21 (2014).

[4] C. L. M. H. Navier, Mémoire sur les lois du mouvement des fluids, Mem. Acad. Sci. Int. Fr. 6, 389 (1823).

[5] A. Steinberger, C. Cottin-Bizonne, P. Kleimann, and E. Charlaix, High friction on a bubble mattress, Nat. Mater. 6, 665 (2007).

[6] D. Legendre and C. Colin, Enhancement of wall friction by fixed cap bubbles, Phys. Fluids 20, 051704 (2008).

[7] J. Hyväluoma and J. Harting, Slip flow over structured surfaces with entrapped microbubbles, Phys. Rev. Lett. 100, 246001 (2008).

[8] D. Crowdy, Slip length for longitudinal shear flow over a dilute periodic mattress of protruding bubbles, Phys. Fluids 22, 121703 (2010).

[9] C. J. Teo and B. C. Khoo, Flow past superhydrophobic surfaces containing longitudinal grooves: effects of interface curvature, Microfluid. Nanofluid. 9, 499 (2010).

[10] C.-O. Ng and C. Y. Wang, Effective slip for Stokes flow over a surface patterned with two- or three-dimensional protrusions, Fluid Dyn. Res. 43, 065504 (2011).

[11] A. M. J. Davis and E. Lauga, Geometric transition in friction for flow over a bubble mattress, Phys. Fluids 21, 011701 (2009). 
[12] J. Hyväluoma, C. Kunert, and J. Harting, Simulations of slip flow on nanobubble-laden surfaces, J. Phys. Condens. Mat. 23, 184106 (2011).

[13] E. Karatay, A. S. Haase, C. W. Visser, C. Sun, D. Lohse, P. A. Tsai, and R. G. H. Lammertink, Control of slippage with tunable bubble mattresses., P. Natl Acad. Sci. USA 110, 8422 (2013).

[14] A. S. Haase, E. Karatay, P. A. Tsai, and R. G. H. Lammertink, Momentum and mass transport over a bubble mattress: the influence of interface geometry, Soft Matter 9, 8949 (2013).

[15] C. J. Teo and B. C. Khoo, Effects of interface curvature on Poiseuille flow through microchannels and microtubes containing superhydrophobic surfaces with transverse grooves and ribs, Microfluid. Nanofluid. 17, 891 (2014).

[16] H. H. Winter, in AIChE modular instruction series C, edited by J. R. Gordon (American Institute of Chemical Engineers, New York, 1987), Vol. 7, Chap. C7.4, pp. 27-34.

[17] R. B. Bird, W. E. Stewart, and E. N. Lightfoot, Transport phenomena, 2nd ed. (John Wiley \& Sons, New York, 2007).

[18] H. K. Moffatt, Viscous and resistive eddies near a sharp corner, J. Fluid Mech. 18, 1 (1964).

\section{Appendix}

\section{Dissipation rate function}

The Moffatt solution [18] for flow of a viscous fluid near a sharp corner is written as

$$
\psi=A f(\phi) \tilde{r}^{\lambda}
$$

with $A$ being a certain prefactor and $f(\phi)$ being a function of the form

$$
f(\phi)=P \cos \lambda \phi+Q \sin \lambda \phi+R \cos (\lambda-2) \phi+S \sin (\lambda-2) \phi
$$

where $P, Q, R$ and $S$ are arbitrary constants, determined by the boundary conditions. Using this solution, we can derive a function for the dissipation rate near the corner. In polar coordinates, the dissipation rate $\tilde{\Phi}$ is given by

$$
\tilde{\Phi}=2\left[\left(\frac{\partial \tilde{u}_{r}}{\partial \tilde{r}}\right)^{2}+\left(\frac{1}{\tilde{r}} \frac{\partial \tilde{u}_{\phi}}{\partial \phi}+\frac{\tilde{u}_{r}}{\tilde{r}}\right)^{2}\right]+\left[\tilde{r} \frac{\partial}{\partial \tilde{r}}\left(\frac{\tilde{u}_{\phi}}{\tilde{r}}\right)+\frac{1}{\tilde{r}} \frac{\partial \tilde{u}_{r}}{\partial \phi}\right]^{2} .
$$


Since $\psi=A f(\phi) \tilde{r}^{\lambda}$,

$$
\begin{aligned}
& \tilde{u}_{r}=\frac{1}{\tilde{r}} \frac{\partial \psi}{\partial \phi}=A f^{\prime} \tilde{r}^{\lambda-1}, \\
& \tilde{u}_{\phi}=-\frac{\partial \psi}{\partial \tilde{r}}=-A f \lambda \tilde{r}^{\lambda-1} .
\end{aligned}
$$

This ultimately results in the following expression for $\tilde{\Phi}$ :

$$
\begin{aligned}
\tilde{\Phi} & =A^{2}\left[2 f^{\prime 2}(\lambda-1)^{2}+2 f^{\prime 2}(1-\lambda)^{2}+\left(f^{\prime \prime}-f \lambda(\lambda-2)\right)^{2}\right] \tilde{r}^{2 \lambda-4} \\
& =A^{2} g(\phi) \tilde{r}^{2 \lambda-4} .
\end{aligned}
$$

\section{Stream function for shear flow over bubble mattress}

For shear flow over a bubble mattress in the dilute limit, i.e. $\varepsilon \ll 1$, Davis and Lauga [11] solved the velocity profile. The stream function $\psi(\xi, \eta)$ is derived in a bipolar coordinate system with

$$
\begin{aligned}
& x=\frac{c \sinh \xi}{\cosh \xi+\cos \eta}, \\
& y=\frac{c \sin \eta}{\cosh \xi+\cos \eta} .
\end{aligned}
$$

Here, $c=L_{g} / 2$. The stream function $\psi$, which far away from the surface equals $\psi=\gamma y^{2} / 2$, is written as

$$
\begin{aligned}
\psi & =\gamma c^{2}\left[\frac{1 / 2 \sin ^{2} \eta}{(\cosh \xi+\cos \eta)^{2}}-\frac{\int_{0}^{\infty} f(s, \eta) \cos s \xi \mathrm{d} s}{\cosh \xi+\cos \eta}\right] \\
f & =-A(s) \sin \eta \frac{\sinh s(\pi-\eta)}{s} \\
& +B(s)\left[\cos \eta \frac{\sinh s(\pi-\eta)}{s}+\sin \eta \cosh s(\pi-\eta)\right]
\end{aligned}
$$

$\gamma$ being the shear rate. Here,

$$
\begin{aligned}
A(s) & =\frac{s}{\sinh 2 s(\pi-\vartheta)+s \sin 2 \vartheta} \\
& \times\left[\cos 2 \vartheta+\frac{s \sin 2 \vartheta \cosh s \pi+\sinh s(\pi-2 \vartheta)}{\sinh s \pi}\right],
\end{aligned}
$$

and

$$
B(s)=\frac{s \sin 2 \vartheta}{\sinh 2 s(\pi-\vartheta)+s \sin 2 \vartheta}
$$


Besides the integration variable $s$, both $A$ and $B$ only depend on the bubble protrusion angle $\vartheta$. The effective slip length of the bubble mattress is expressed as

$$
\frac{2 b}{L_{g}}(\vartheta)=\varepsilon \pi \int_{0}^{\infty} A(s) \mathrm{d} s .
$$





\title{
CHAPTER 4
}

\section{Inelastic non-Newtonian flow over heterogeneously slippery surfaces}

\begin{abstract}
In this study, we investigated inelastic non-Newtonian fluid flow over heterogeneously slippery surfaces. First, we simulated the flow of aqueous xanthan gum solutions over a bubble mattress, which is a superhydrophobic surface consisting of transversely positioned no-slip walls and no-shear gas bubbles. The results reveal that for shear-thinning fluids wall slip can be increased significantly, provided that the system is operated in the shear-thinning regime. For a $0.2 \mathrm{wt} \%$ xanthan gum solution with a power-law index of $n=0.4$, the numerical results indicate that wall slip can be enhanced 3.2 times when compared to a Newtonian liquid. This enhancement factor was also predicted from a theoretical analysis, which gave an expression for the maximum slip length that can be attained over flat, heterogeneously slippery surfaces. Although this equation was derived for a no-slip/no-shear unit length that is much larger than the typical size of the system, we found that it can also be used to predict the enhancement in the regime where the slip length is proportional to the size of the no-shear region or the bubble width. The results could be coupled to the hydrodynamic development or entrance length of the system, as maximum wall slip is only reached when the fluid flow can fully adapt to the no-slip and no-shear conditions at the wall.
\end{abstract}

This chapter has been submitted for publication as A.S Haase, J.A. Wood, L.M.J. Sprakel and R.G.H. Lammertink, Inelastic non-Newtonian flow over heterogeneously slippery surfaces (2016). 


\subsection{Introduction}

To enhance transport phenomena in microfluidic and nanofluidic systems, surfaces can be made slippery. The slipperiness of the surface not only leads to lower friction at the wall, and therefore higher flow rates for a given driving force, but also increases convection near the wall, improving transport of, for example, heat or mass. Navier's slip condition [1] is commonly used to quantify the amount of wall slip. This condition states that the slip velocity $u_{b}$ times a certain friction factor $k$ equals the shear force $\mu \partial_{n} u$ at the wall, i.e.

$$
k u_{b}=\mu \frac{\partial u}{\partial n} .
$$

The ratio $\mu / k=b$ is referred to as the slip length and quantifies the amount of wall slip. For smooth surfaces displaying intrinsic wall slip - the fluid molecules adjacent to the substrate have a finite velocity relative to the wall - typical slip lengths are of the order of tens of nanometres [2]. Much larger slip lengths are observed for superhydrophobic surfaces, of the order of micrometres $[3,4]$, although this concerns effective wall slip. For these superhydrophobic surfaces, which contain (sub)microscale wall structures in which usually a gas is entrapped, the liquid is flowing over an array of no-slip regions and (nearly) shear-free gas bubbles. The effective slip length therefore represents the slip length that would be observed at scales much larger than those of the surface structures.

The fact that superhydrophobic surfaces can be used to generate slip lengths on the scale of a typical microfluidic system has led to many theoretical, numerical and experimental studies in which the relationship between surface geometry and wall slip has been investigated [3-6]. These studies, however, mainly concern slip flow of Newtonian liquids over superhydrophobic surfaces.

The existence of wall slip in systems without superhydrophobic or patterned surfaces has been studied for a much longer time for a wide range of complex fluids, such as polymer melts, (bio)polymers solutions, suspensions and gels [7-11]. The slip mechanisms in such systems can vary. Three possible apparent slip mechanisms are commonly considered in the literature, which all differ from true or intrinsic wall slip [5]. The first mechanism is the formation of a low-viscosity slip or depletion layer near the wall [9]. This layer, formed due to migration of the polymer chains towards the bulk of the flow, then acts as a lubrication layer over which the more viscous polymeric solution flows. The thickness of this layer is typically of the order of the size of the polymer coil, i.e. $\mathcal{O}(\mathrm{nm})$. Second, slippage can be the result of breaking the adhesion between the polymer chains and the substrate, thereby reducing the friction at the wall [12-14]. Third, disentanglement of polymers chains in the bulk from the chains at the wall, i.e. cohesive slip, can also give rise to slippage $[14,15]$. Both the second and third 
mechanism require the shear stress to be larger than a certain critical value.

True wall slip is less commonly observed, which results from the fact that essentially all surfaces are rough at an atomic scale. It is for this reason that in most situations the no-slip boundary condition holds [16-18]. Sanchez-Reyes and Archer [19] showed for a shear-thinning polymer solution that wall roughness can be employed to suppress (the effects of) wall slip, which in general is an undesirable phenomenon in the field of rheology [10].

It is believed that slip resulting from the formation of a lubrication layer is the most important and relevant slip mechanism for most polymeric systems, in particular for dilute polymer solutions $[7,9,10,20]$. The most simple representation of this mechanism is that there is a thin layer of a low viscosity solvent near the wall, over which the bulk fluid with the original polymer concentration is flowing. In that case, the apparent slip length $b_{a}$ equals to

$$
b_{a}=\delta\left(\frac{\mu_{s}}{\mu_{l}}-1\right),
$$

where $\delta$ is the thickness of the depletion layer, and $\mu$ is the viscosity of the (polymer) solution $(s)$ and of the liquid in the lubrication layer $(l)$. However, as pointed out by Barnes [9], in reality the polymer concentration gradually changes between the wall and the bulk of the fluid. Tuinier and Taniguchi [21] derived the following expression for the slip length in the case a depletion layer is formed near the wall:

$$
b_{a}=-\delta \sqrt{[\mu] c_{b}} \arctan \left(\sqrt{[\mu] c_{b}}\right) .
$$

This converges to the limit of $b=-\delta[\mu] c_{b}$ when $[\mu] c_{b}$ is very small. The depletion layer thickness $\delta$ approximately corresponds to the radius of gyration $R_{g}$ of the polymer in the case of a dilute solution. $[\mu]$ is the intrinsic viscosity of the solution, given in volume per mass $(\mathrm{L} / \mathrm{g})$, and $c_{b}$ is the bulk polymer concentration.

The extent of migration from the wall towards the bulk, and therefore the depletion layer thickness $\delta$, depends on the polymer concentration $[22,23]$ and the shear rate [24-26]. The results are ambiguous, however. Ausserré et al. [22] showed that for aqueous solutions of xanthan gum, a commonly used polysaccharide to study non-Newtonian fluid behaviour, the depletion layer thickness $\delta$ near a non-adsorbing fused silica wall is maximum $2.2 \mathrm{~nm}$. The thickness decreases rapidly when $c>c^{*}$. The critical concentration $c^{*} \sim 1 /[\mu]$ indicates the transition of the dilute to the semi-dilute regime. The transition of the semi-dilute to the concentrated regime is denoted by $c^{* *}$. For xanthan gum, these critical concentrations are $c^{*} \sim 0.2 \mathrm{~g} / \mathrm{L}$ and $c^{* *} \sim 1 \mathrm{~g} / \mathrm{L}[27-31]$.

When evaluating Eq. (4.3), given that the radius of gyration $R_{g}$ is of the order of $100 \mathrm{~nm}$ for xanthan gum [32,33], we predict an apparent slip length of $b_{a} \sim 0.4 \mu \mathrm{m}$ for an aqueous $0.2 \mathrm{wt} \%$ xanthan gum solution. Compared to the 
typical slip lengths found in slippery, superhydrophobic systems with microscale surface structures (as for those surfaces typically $b_{f} / L \sim \mathcal{O}(1), L$ being the typical length of the surface structures [34-36]), the estimated apparent slip length is one order of magnitude smaller than the measured effective slip lengths, i.e. $b_{a}<b_{f}$. Direct measurements using NMR velocimetry of the shear velocity of aqueous xanthan gum solutions up to $1 \mathrm{wt} \%$ near a PMMA or glass wall indeed suggest that apparent wall slip is absent or otherwise very small [37-39].

Although xanthan gum solutions do not appear to show apparent wall slip due to depletion effects, these solutions are shear-thinning: the viscosity changes with shear rate [27-31]. The dependency of viscosity on shear may lead to some interesting behaviour near textured substrates like superhydrophobic surfaces. The heterogeneity of these surfaces leads to local variations in the shear rate. For flow over superhydrophobic surfaces in the Cassie-Baxter state, i.e. when a gas is entrapped in the surface structures, the conditions at the hybrid bottom wall are a pattern of no-slip and no-shear. Various studies considering flow over a superhydrophobic surface reported large variations in the shear stress distribution at the wall [40-44]. This implies that for flow of non-Newtonian liquids, such as xanthan gum solutions, over superhydrophobic surfaces the local viscosity also varies near the wall. It immediately follows from Eq. (4.1) that this will affect the local and therefore the effective slip length.

The effect of a slippery wall with varying slip length on pressure-driven nonNewtonian fluid flow has been investigated analytically by Pereira [45] and by using molecular dynamics simulations by Dhondi et al. [46]. They concluded that for such surfaces, by looking at the wall-induced transverse flows, mixing is enhanced for shear-thickening fluids and suppressed for shear-thinning fluids. Applying an oscillating body force was predicted to increase transverse flow. Vayssade et al. [47] showed for aqueous suspensions of microgel particles that heterogeneity in slip conditions at the wall has a substantial influence on the velocity profile, in particular for confined systems. Recently, Broboana et al. [48] investigated both Newtonian and non-Newtonian shear flow between the patterned and liquid-filled parallel plates of a rheometer. Their results underline that surface heterogeneity leads to local variations in shear stress and viscosity, in particular for a shear-thinning liquid.

Flows of complex fluids over microscale patterned substrates have hardly been studied to date, despite the fact that non-Newtonian liquids are encountered frequently in the field of microfluidics. Examples include flows of polymer solutions $[49,50]$, blood [51] and other biological samples [52] in (diagnostic) microfluidic systems. In this study, we investigate slip flow of an inelastic non-Newtonian liquid over a superhydrophobic surface with transverse grooves and ribs - a surface often referred to as a bubble mattress. Since slip caused by wall depletion effects seems to be insignificant for aqueous xanthan gum solutions, we used these solutions 


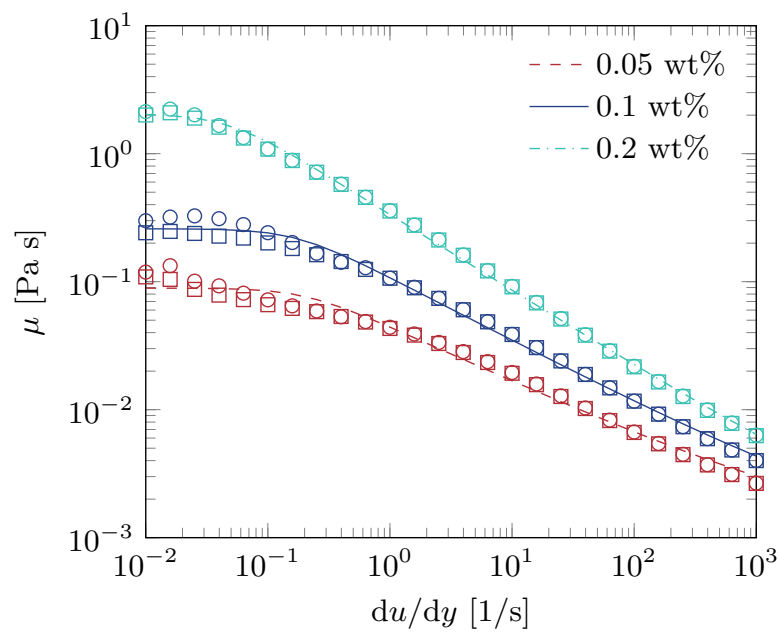

Figure 4.1 - Viscosity $\mu$ for multiple xanthan gum solutions. The lines are the fitted Carreau expressions to the experimental data (the circles and squares correspond to different samples).

as model fluids. The numerical results reveal that shear-thinning behaviour can enhance effective wall slip over superhydrophobic surfaces. The maximum increase in wall slip compared to Newtonian liquids could be predicted from a theoretical analysis. We also discuss the relationship between wall slip and the hydrodynamic development of the local velocity profile.

\subsection{Methodology}

\subsubsection{Flow curves of xanthan gum solutions}

Aqueous solutions with $0.05,0.1$ and $0.2 \mathrm{wt} \%$ xanthan gum (XG) from Xanthomonas campestris (G1253, Sigma-Aldrich) were prepared in MilliQ water. Since $c^{*} \sim 0.2 \mathrm{~g} / \mathrm{L}$ and $c^{* *} \sim 1 \mathrm{~g} / \mathrm{L}[27-31]$, the xanthan gum concentrations $c$ of our aqueous solutions are in the semi-dilute regime. Visco-elastic effects are not considered in this study, which is supported by the fact that dilute xanthan gum solutions are relatively inelastic $[53,54]$. The shear-dependent viscosity of the xanthan gum solutions was characterised using an Anton Paar MCR 302 rheometer equipped with a DG26.7 double gap concentric cylinder geometry at an temperature of $25^{\circ} \mathrm{C}$. For each xanthan gum concentration, two samples were used for the rheological experiments. The results are plotted in Fig. 4.1. 
Table 4.1 - Fit parameters (95\% confidence interval) for the Carreau expression for various xanthan gum solutions. $\mu_{\infty}$ was set to the viscosity of water at $25^{\circ} \mathrm{C}$, which is $8.9 \times 10^{-4} \mathrm{~Pa} \mathrm{~s}$.

\begin{tabular}{lllll}
\hline $\mathrm{c}[\mathrm{wt} \%]$ & $\mu_{0}[\mathrm{~Pa} \mathrm{~s}]$ & $\lambda[\mathrm{s}]$ & $n$ & $R^{2}$ \\
\hline 0.05 & $0.0890 \pm 0.0078$ & $5.0 \pm 1.8$ & $0.563 \pm 0.024$ & 0.9855 \\
0.1 & $0.259 \pm 0.017$ & $5.9 \pm 1.4$ & $0.503 \pm 0.016$ & 0.9946 \\
0.2 & $2.05 \pm 0.12$ & $21.2 \pm 3.1$ & $0.406 \pm 0.008$ & 0.9988 \\
\hline
\end{tabular}

The flow curves are described using the Carreau expression:

$$
\mu=\mu_{\infty}+\left(\mu_{0}-\mu_{\infty}\right)\left(1+\left(\lambda \frac{\mathrm{d} u}{\mathrm{~d} y}\right)^{2}\right)^{(n-1) / 2} .
$$

To determine the coefficients of this constitutive equation, the measurement data was fit in a least-squares sense using MATLAB. Fitting was performed for $\log _{10} \mu=f\left(\log _{10}(\mathrm{~d} u / \mathrm{d} y)\right)$, with the normality of residuals checked using a normal probability plot. The fit parameters and confidence intervals are presented in Table 4.1 .

\subsubsection{Numerical method}

The effect of shear-thinning (non-viscoelastic) behaviour on effective wall slip was investigated numerically by using a geometry with bubbles and grooves positioned perpendicular to the flow direction. The geometry, which is commonly referred to as a bubble mattress, is illustrated in Fig. 4.2. The model is two-dimensional, as it is assumed that the length of the bubbles and grooves in the third dimension is very long compared to the channel height $H$. The model is also periodic, consisting of $N=5$ bubble units with a unit length $L$. The size of the bubbles $L_{g}$ is related to the surface porosity $\varepsilon=L_{g} / L$. Throughout this study, the porosity is fixed at $\varepsilon=1 / 2$. The gas-liquid interface curvature, which is known to affect the amount of wall slip $[44,55,56]$, is varied by changing the bubble protrusion angle $\vartheta$.

Fluid flow over the heterogeneously slippery surface is described by the continuity and the Navier-Stokes equation,

$$
\begin{gathered}
\nabla \cdot \mathbf{u}=0, \\
\rho(\mathbf{u} \cdot \nabla \mathbf{u})=-\nabla p+\nabla \cdot\left[\mu\left(\nabla \mathbf{u}+(\nabla \mathbf{u})^{\mathrm{T}}\right)\right],
\end{gathered}
$$

where $\mathbf{u}=(u, v)$. The convection terms are included in the computations, as the 
(a) $\mu[\mathrm{Pa} \mathrm{s}]$

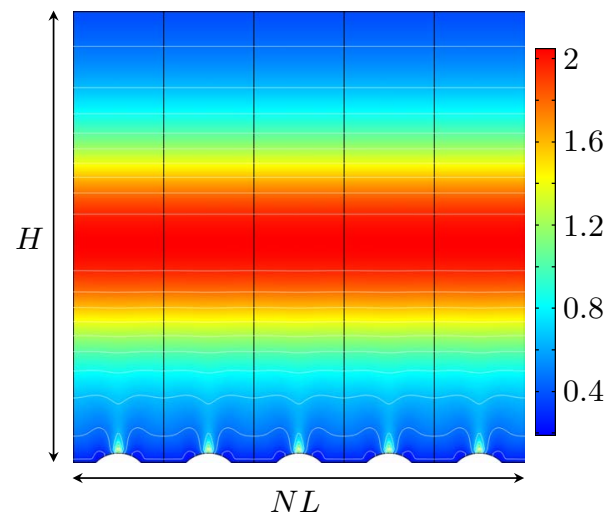

(b) $|\mathbf{u}|[\mu \mathrm{m} / \mathrm{s}]$

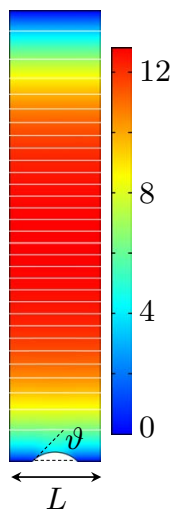

(c) $\mathrm{d} \mathbf{u} / \mathrm{d} \mathbf{x}[1 / \mathrm{s}]$

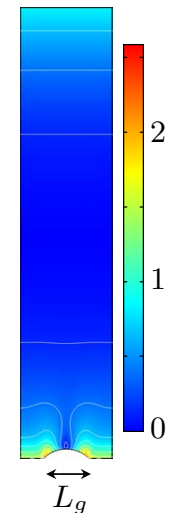

Figure 4.2 - Example of the periodic bubble mattress model, consisting of $N=5$ bubble units with height $H=100 \mu \mathrm{m}$, unit length $L=20 \mu \mathrm{m}$ and bubble width $L_{g}=10 \mu \mathrm{m}$. The bubble protrusion angle of, in this case, $\vartheta=45^{\circ}$ defines the gas-liquid interface curvature. The surface porosity is $\varepsilon=1 / 2$. The plot in $(a)$ shows the local viscosity of a $0.2 \mathrm{wt} \%$ xanthan gum solution for an applied average liquid velocity of $u_{a v}=10^{-5} \mathrm{~m} / \mathrm{s}$. In $(b)$ and $(c)$ are the velocity magnitude $|\mathbf{u}|$ and the shear rate $\mathrm{d} \mathbf{u} / \mathrm{d} \mathbf{x}$ with $\mathbf{x}=(x, y)$ plotted for a single unit.

average velocity is sufficiently high to let $R e>1$. The liquid density is assumed to be that of pure water [57], i.e. $\rho=10^{3} \mathrm{~kg} / \mathrm{m}^{3}$.

The numerical model is periodic, implying that left (source) and right (destination) boundary of the computational domain are equal in terms of velocity, i.e.

$$
\left\{\begin{array}{l}
\mathbf{u}(0, y)=\mathbf{u}(N L, y), \\
p(0, y)=p(N L, y) .
\end{array}\right.
$$

The flow is pressure-driven, and therefore

$$
p(0, y)-p(N L, y)=-N L \nabla p \text {. }
$$

The pressure is specified at an arbitrary value and location in the domain. The pressure gradient is determined iteratively in order to fix the average velocity $u_{a v}$ at a value of 1 . Except for the slippery bubbles, the no-slip boundary condition is applied to the upper and lower wall:

$$
\mathbf{u}=\mathbf{0} \text {. }
$$

The bubble surfaces are impermeable and perfectly slippery or shear-free, which 
is described by

$$
\left\{\begin{array}{l}
\mathbf{n} \cdot \mathbf{u}=0 \\
{\left[-p \mathbf{I}+\mu\left(\nabla \mathbf{u}+(\nabla \mathbf{u})^{\mathrm{T}}\right)\right] \cdot \mathbf{n}=\mathbf{0} .}
\end{array}\right.
$$

The isotropic viscosity $\mu$ of the fluid is described by the Carreau expression given in Eq. (4.4). This equation ignores elastic, that is time-dependent, effects. This means that the relaxation of the fluid is instantaneous and that the fluid is perfectly viscous.

The equations were solved numerically using COMSOL Multiphysics 5.2. The standard relative tolerance was $1 \times 10^{-3}$. P2+P1 discretisation (second order elements for velocity and first order elements for pressure) was used to solve the Navier-Stokes equations. The mesh was refined near the walls. The complete mesh consisted of approximately 55000 domain elements and about 1200 boundary elements in the vicinity of the bubbles.

\subsubsection{Determination of the effective slip length}

After solving the numerical model, the effective slip length $b$ was determined by relating the numerically obtained average liquid velocity $u_{a v}$ and pressure gradient $\mathrm{d} p / \mathrm{d} x$ to the slip length that would give the same average velocity and pressure gradient for a (one-sided) homogeneously slippery channel. To obtain the relationship between $u_{a v}, \mathrm{~d} p / \mathrm{d} x$ and $b$ for a homogeneously slippery channel, the Navier-Stokes equation for uni-directional fluid flow is solved:

$$
0=-\frac{\mathrm{d} p}{\mathrm{~d} x}+\frac{\mathrm{d}}{\mathrm{d} y}\left(\mu \frac{\mathrm{d} u}{\mathrm{~d} y}\right)
$$

To make the governing equation dimensionless, the following variables are introduced: $\tilde{y}=y / H, \tilde{x}=x / H, \tilde{u}=u / U$ with $U=-\left(H^{2} / \mu_{0}\right)(\mathrm{d} P / \mathrm{d} x), \tau=$ $\lambda U / H$ and $\tilde{\mu}=\mu_{\infty} / \mu_{0}$ (note that for parallel plane Poiseuille flow $u_{a v}=$ $\left.-\left(H^{2} / 12 \mu\right)(\mathrm{d} P / \mathrm{d} x)\right)$. The dimensionless Navier-Stokes equation then becomes

$$
0=1+\frac{\mathrm{d}}{\mathrm{d} \tilde{y}}\left\{\left[\tilde{\mu}+(1-\tilde{\mu})\left(1+\left(\tau \frac{\mathrm{d} \tilde{u}}{\mathrm{~d} \tilde{y}}\right)^{2}\right)^{(n-1) / 2}\right] \frac{\mathrm{d} \tilde{u}}{\mathrm{~d} \tilde{y}}\right\}
$$

This equation is solved by a second-order-accurate finite difference scheme combined with the MATLAB fsolve algorithm. As an initial guess for the velocity profile, the profile for Poiseuille flow with one-sided slip was used. When solving the non-Newtonian velocity profile for a range of average velocities and pressure gradients for a homogeneously slippery channel with a specified height $H$, an interpolation map $u_{a v}=f(\mathrm{~d} p / \mathrm{d} x, b)$ was obtained. Solving the COMSOL model for a specified geometry and average velocity gives the pressure gradient over the 

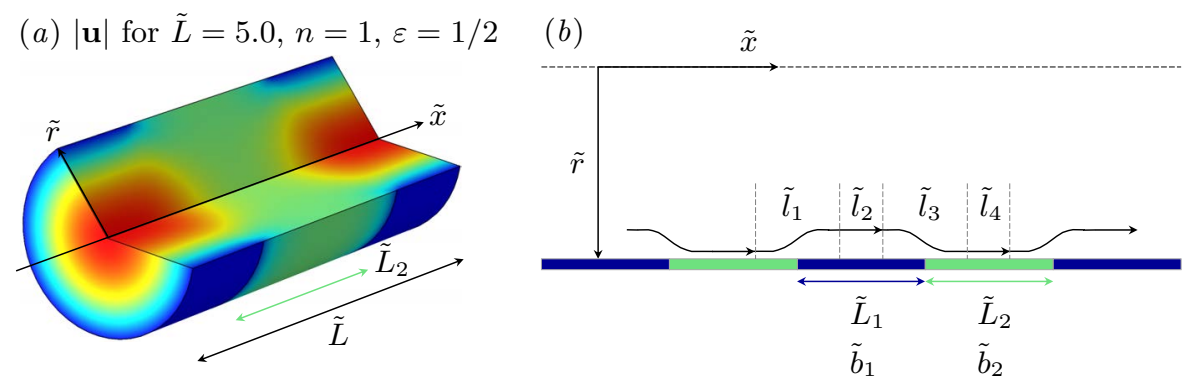

Figure 4.3 - In (a) an example of the cylindrical geometry is shown that is used to investigate the relationship between the effective slip length $\tilde{b}=b / R$ and the relative bubble unit confinement $\tilde{L}=L / R$. The color scale varies from red for $|\mathbf{u}|=2$ to dark blue for $|\mathbf{u}|=0$. In $(b)$, a schematic of the system is given. The flow pattern over the no-slip $\left(\tilde{L}_{1}\right.$ with $\left.\tilde{b}_{1}=0\right)$ and no-shear regions $\left(\tilde{L}_{2}\right.$ with $\left.\tilde{b}_{2}=\infty\right)$ can be divided into four different subregions: $\tilde{l}_{i}$ with $i=[1 \ldots 4]$. When $\tilde{L} \rightarrow \infty$, the effective pressure drop in the domain is dominated by the no-slip region $\tilde{l}_{2}$.

bubble mattress. Subsequently the interpolation map was used to determine the effective slip length for Carreau flow over the bubble mattress by interpolation using the MATLAB function interp2 (interpolation based on a cubic spline).

\subsection{Limiting values for effective wall slip}

In this section, we derive the theoretical limit for the effective slip length for non-Newtonian flow over a heterogeneously slippery surface consisting of an alternating pattern of no-slip and no-shear regions positioned perpendicular to the flow direction. Although the shear-thinning behaviour of fluids like xanthan gum solutions are well described by constitutive relations that also capture the zero-shear and infinite-shear Newtonian plateaus, the shear-thinning region is often described by a power law:

$$
\mu=\mu_{0}\left(\frac{\partial u}{\partial r}\right)^{n-1}
$$

Because of its mathematical simplicity, we will describe the derivation of the theoretical limit for effective wall slip for a power law fluid. For the same reason, we change from a planar to a cylindrical coordinate system (see Fig. 4.3(a) for a illustration of the cylindrical system). The derived equations will be analogous for a planar geometry, and can therefore also be used to predict approximately the maximum slip length for the planar system shown in Fig. 4.2 (as we will demonstrate later in Sec. 4.4.2).

A schematic of the system is given in Fig. 4.3(b). Here, we can distinguish 
four different regions with a length $\tilde{l}_{i}=l_{i} / R$, with $i=[1 \ldots 4]$. As indicated in Fig. 4.3, in the regions 1 and 3 the flow is developing and therefore crosses the no-slip region $\tilde{L}_{1}=L_{2} / R$ with $\tilde{b}_{1}=0$ and the no-shear region $\tilde{L}_{2}$ with $\tilde{b}_{2}=\infty$. The total length of a no-slip/no-shear unit equals $\tilde{L}=\tilde{L}_{1}+\tilde{L}_{2}$. In the case the flow over these regions is not fully developed, we find that $\tilde{l}_{2}, \tilde{l}_{4}=0$. Note that, since we are considering Stokes flow, $\tilde{l}_{1}=\tilde{l}_{3}$. On the other hand, when the no-slip and no-shear regions would be infinitely long compared to the tube radius, i.e. $\tilde{L}_{1}, \tilde{L}_{2} \rightarrow \infty$, we find that $\left(\tilde{l}_{1}, \tilde{l}_{3}\right) /\left(\tilde{l}_{2}, \tilde{l}_{4}\right) \rightarrow 0$. The flow is hydrodynamically fully developed and has completely adapted to the local boundary conditions.

In order to obtain the effective slip length for this system, one cannot take the spatial average of the local slip length, as this will yield an infinite slip length: $\tilde{b}_{f} \neq\left(\tilde{L}_{1} \tilde{b}_{1}+\tilde{L}_{2} \tilde{b}_{2}\right) /\left(\tilde{L}_{1}+\tilde{L}_{2}\right)=\infty$. However, one can define an effective pressure gradient, which subsequently can be used to obtain the effective slip length:

$$
\left(\frac{\partial \tilde{p}}{\partial \tilde{x}}\right)_{f}=\sum_{i=1}^{4} \tilde{l}_{i}\left(\frac{\partial \tilde{p}}{\partial \tilde{x}}\right)_{i} / \sum_{i=1}^{n} \tilde{l}_{i}
$$

In case of a developing flow, i.e. $\tilde{l}_{2}, \tilde{l}_{4}=0$, this equation cannot be evaluated $a$ priori, since the pressure drop over the regions 1 and 3 is not known. However, when $\tilde{L}_{1}, \tilde{L}_{2} \rightarrow \infty$ and thus $\sum \tilde{l}_{i} \rightarrow \tilde{l}_{2}+\tilde{l}_{4}$, we find that

$$
\left(\frac{\partial \tilde{p}}{\partial \tilde{x}}\right)_{f}=\frac{1}{\tilde{l}_{2}+\tilde{l}_{4}}\left[\tilde{l}_{2}\left(\frac{\partial \tilde{p}}{\partial \tilde{x}}\right)_{2}+\tilde{l}_{4}\left(\frac{\partial \tilde{p}}{\partial \tilde{x}}\right)_{4}\right] \text {. }
$$

Considering that the local pressure gradient for a no-shear region equals zero and that the surface porosity can be approximated by $\varepsilon=\tilde{L}_{2} /\left(\tilde{L}_{1}+\tilde{L}_{2}\right) \sim \tilde{l}_{4} /\left(\tilde{l}_{2}+\tilde{l}_{4}\right)$, this finally yields the following expression for the effective pressure gradient:

$$
\left(\frac{\partial \tilde{p}}{\partial \tilde{x}}\right)_{f}=\frac{\tilde{l}_{2}}{\tilde{l}_{2}+\tilde{l}_{4}}\left(\frac{\partial \tilde{p}}{\partial \tilde{x}}\right)_{2}=(1-\varepsilon)\left(\frac{\partial \tilde{p}}{\partial \tilde{x}}\right)_{2} .
$$

The pressure gradient for the flow of a power-law fluid through a non-slippery tube can be evaluated, and thus an expression can be found for the maximum slip length that can be achieved.

The velocity profile of a power-law fluid through a tube with a slippery wall having an uniform slip length $b$ and a pressure drop $\partial p / \partial x$ is given by

$$
u=R\left(-\frac{R}{2 \mu_{0}} \frac{\partial p}{\partial x}\right)^{1 / n}\left[\frac{1}{1 / n+1}\left(1-\left(\frac{r}{R}\right)^{1 / n+1}\right)+\frac{b}{R}\right]
$$


The average velocity then equals

$$
u_{a v}=R\left(-\frac{R}{2 \mu_{0}} \frac{\partial p}{\partial x}\right)^{1 / n}\left[\frac{1}{1 / n+3}+\frac{b}{R}\right] .
$$

Both equations can be made dimensionless using $b=\tilde{b} R, x=\tilde{x} R, u=\tilde{u} u_{a v}$ and $\partial p / \partial x=\left(R / \mu_{0}\right)\left(R / u_{a v}\right)^{n}(\partial \tilde{p} / \partial \tilde{x})$, yielding

$$
\tilde{u}=\left(-\frac{1}{2} \frac{\partial \tilde{p}}{\partial \tilde{x}}\right)^{1 / n}\left[\frac{1}{1 / n+1}\left(1-\tilde{r}^{1 / n+1}\right)+\tilde{b}\right]
$$

for the velocity profile, and

$$
1=\left(-\frac{1}{2} \frac{\partial \tilde{p}}{\partial \tilde{x}}\right)^{1 / n}\left[\frac{1}{1 / n+3}+\tilde{b}\right]
$$

for the average dimensionless velocity which equals one. Thus, for a system with an average velocity $u_{a v}=1$ having a known pressure gradient, we can calculate the slip length by

$$
\tilde{b}=\left(-\frac{1}{2} \frac{\partial \tilde{p}}{\partial \tilde{x}}\right)^{-1 / n}-\frac{1}{1 / n+3} .
$$

We can now evaluate the maximum slip length for a power-law flow through a tube with a heterogeneously slippery wall. As expressed by Eq. (4.16), when $\tilde{L} \rightarrow \infty$, the effective pressure gradient is fully dominated by the gradient over the no-slip regions. This gives, when substituting Eq. (4.20) for $\tilde{b}=0$ into Eq. (4.16), the following relation for the effective pressure gradient:

$$
\left(\frac{\partial \tilde{p}}{\partial \tilde{x}}\right)_{f}=-2(1-\varepsilon)\left(\frac{1}{n}+3\right)^{n}
$$

When using this expression for the effective pressure gradient and substituting that into Eq. (4.21), we finally obtain the following expression for the maximum effective slip length $\tilde{b}_{\max }$ when $\tilde{L} \rightarrow \infty$ :

$$
\tilde{b}_{\max }=\frac{1}{(1 / n+3)(1-\varepsilon)^{1 / n}}-\frac{1}{1 / n+3}=\frac{1-(1-\varepsilon)^{1 / n}}{(1 / n+3)(1-\varepsilon)^{1 / n}} .
$$

In the case of a Newtonian fluid with $n=1$, this yields

$$
\tilde{b}_{\max }=\frac{1}{4} \frac{\varepsilon}{1-\varepsilon} .
$$

Eq. (4.24) for a Newtonian fluid has also been derived by Lauga and Stone [35], although they obtained this result by solving the flow field analytically and 
looking in the asymptotic limit for large distances between the slip regions.

These results are confirmed numerically using COMSOL Multiphysics 5.2 using a periodic cylindrical model consisting of one repeating unit of transverse no-slip and no-shear regions (the regions can be considered as flat gas bubbles with $\left.\vartheta=0^{\circ}\right)$. An example of the model is shown in Fig. 4.3(a) for $\tilde{L}=5.0$.

\subsection{Results and discussion}

\subsubsection{Numerical results}

In this section, we describe and discuss our numerical results. The viscosity of the aqueous xanthan gum solutions is described by the Carreau expression. In Fig. 4.4, the effective slip length $b$ for a $0.2 \mathrm{wt} \%$ xanthan gum solution is plotted as function of the bubble protrusion angle $\vartheta$ for various imposed average velocities. The slip length is normalised by the bubble width $L_{g}$. For small flow rates, i.e. $u_{a v} \leq 10^{-7} \mathrm{~m} / \mathrm{s}$, we observe that the slip length profile coincides with that of water. When increasing the velocity, for all protrusion angles the slip length increases and reaches a maximum when $u_{a v}=10^{-5} \mathrm{~m} / \mathrm{s}$. For larger liquid velocities, this decreases again and converges towards the profile for water. Note that here for liquid velocities larger than $10^{1} \mathrm{~m} / \mathrm{s}$, inertial effects become significant. For that reason, the slip length profile will approach but not fully overlap with that of water (which also starts to change when $u_{a v} \gg 1 \mathrm{~m} / \mathrm{s}$ ).

This dependency of slip length on the velocity can be related to the shearthinning properties of the modelled xanthan gum solution (see Fig. 4.1). In Fig. $4.5(a)$, the slip length $b$ is plotted as function of the average velocity $u_{a v}$ for $\vartheta=1^{\circ}$. For very small flow rates, the shear rates in the fluid flow are very small and the viscosity equals the zero-shear viscosity: $\mu=\mu_{0}$. For intermediate flow rates, the system is operated in the shear-thinning regime of the xanthan gum solutions and the viscosity decreases with increasing flow rates and, consequently, increasing shear rates. As illustrated by Fig. 4.2(a), in this regime the viscosity near the heterogeneously slippery wall strongly varies with position. When the shear rate approaches the upper Newtonian plateau, $\mathrm{d} u / \mathrm{d} y>10^{2}$, the decrease in viscosity with increasing shear rate levels off and the viscosity approaches the infinite shear viscosity: $\mu \rightarrow \mu_{\infty}$.

Note that the critical protrusion angle, the angle for which wall slip becomes negative, also increases when operating the system in the shear-thinning region. It shifts from $63^{\circ}$ when for the zero-shear Newtonian plateau to a maximum value of $79^{\circ}$ for $u_{a v}=10^{-5} \mathrm{~m} / \mathrm{s}$. The increase in slip length with flow rate is considerably smaller, however, for very large positive protrusion angles than for flat bubbles. In case of highly negative angles, there is no enhancement. The liquid in the bubbles is almost stagnant, for which reason the viscosity near the 


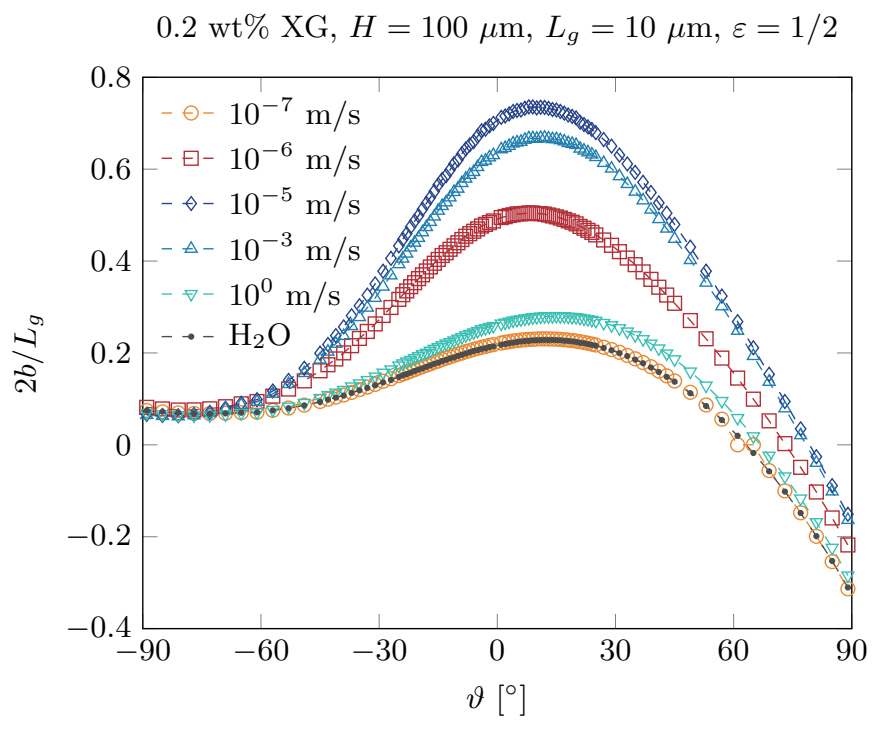

Figure 4.4 - Dimensionless effective slip length $2 b / L_{g}$ for a $0.2 \mathrm{wt} \%$ xanthan gum solution as function of the bubble protrusion angle $\vartheta$ for various average liquid velocities $u_{a v}$. A liquid velocity of $u_{a v}=10^{-5} \mathrm{~m} / \mathrm{s}$ and a viscosity of $\mu=0.89 \mathrm{mPa}$ s was used to calculate the slip length profile for water.

shear-free gas-liquid boundary also is very high: $\mu \sim \mu_{0}$.

We therefore conclude that when the system is operated in one of the Newtonian plateaus, the slip length profile resembles the profile for water. Preliminary experimental results, which are shown in the appendix to this chapter, also confirm this: for very high flow rates $\left(u_{a v}=10^{-1} \mathrm{~m} / \mathrm{s}\right)$ the effective slip lengths for various xanthan gum solutions overlap with those for water. These results also suggest that slip resulting from any depletion related effects are insignificant when compared with the slip induced by the superhydrophobic surface.

In the shear-thinning region, the slip length increases with increasing flow rate, Despite the fact that, as follows from Eq. (4.1), the slip length is a function of both viscosity and shear rate at the wall, in this region $\mu \propto\left(\mathrm{d}_{y} u\right)^{n-1}$ and the viscosity therefore decreases faster than the shear increases when $n<1$. Assuming that the friction factor $k$ is constant, this implies that wall slip should increase. This is confirmed by the numerical results.

The enhancement in slip length, i.e. the slip length for the non-Newtonian xanthan gum solution over that for water, is plotted in Fig. 4.5(b). The more shear-thinning the xanthan gum solutions are (i.e. the smaller $n$ is), the larger the enhancement. For a $0.2 \mathrm{wt} \%$ xanthan gum solution with $n=0.406$, a maximum enhancement factor of 3.2 can be achieved. Note the non-linear relationship 
(a) $H=100 \mu \mathrm{m}, L_{g}=10 \mu \mathrm{m}, \varepsilon=1 / 2, \vartheta=1^{\circ}$

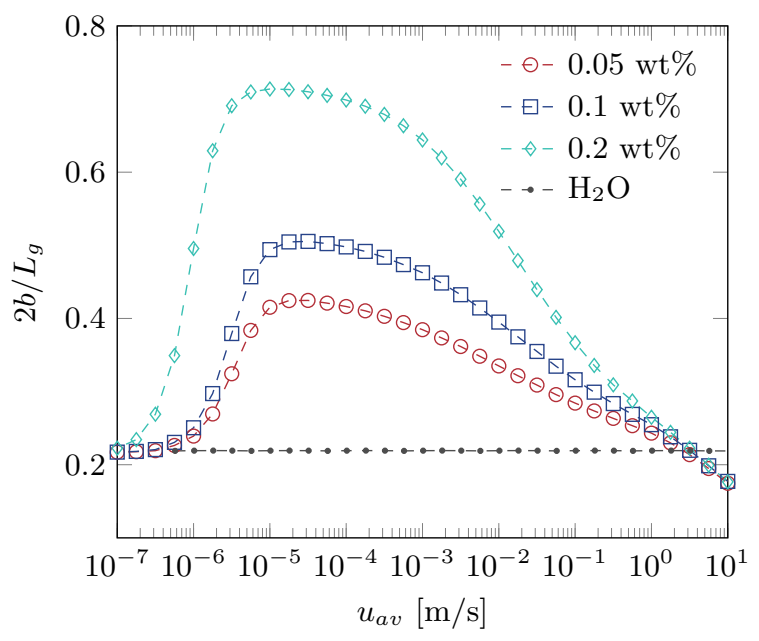

(b) $H=100 \mu \mathrm{m}, L_{g}=10 \mu \mathrm{m}, \varepsilon=1 / 2, \vartheta=1^{\circ}$

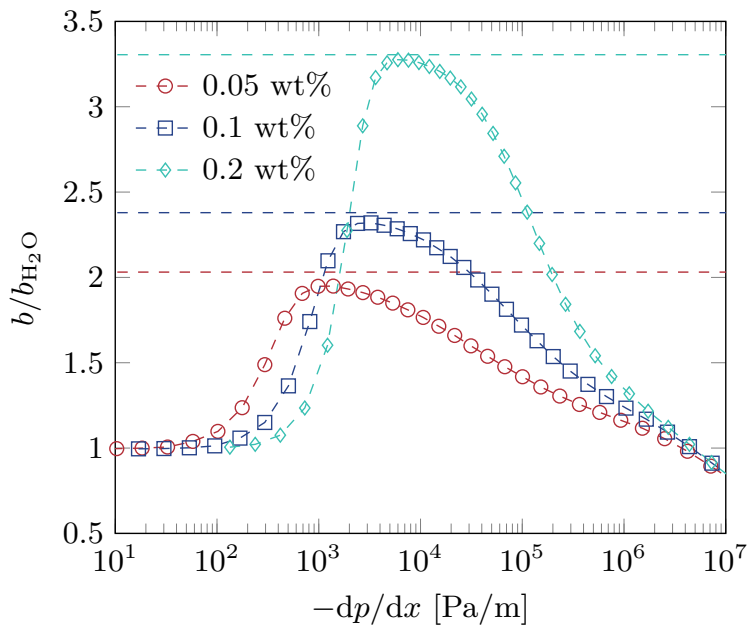

Figure 4.5 - In $(a)$ the effective slip length $2 b / L_{g}$ is plotted as a function of the average liquid velocity $u_{a v}$ for a protrusion angle of $\vartheta=1^{\circ}$ for various xanthan gum solutions. In $(b)$, the slip enhancement factor $b / b_{\mathrm{H}_{2} \mathrm{O}}$ for $\vartheta=1^{\circ}$ is plotted as a function of the effective pressure gradient $\mathrm{d} p / \mathrm{d} x$, where $b_{\mathrm{H}_{2} \mathrm{O}}$ has been determined for $u_{a v}=10^{-5}$ $\mathrm{m} / \mathrm{s}$. The horizontal dashed lines are the analytically predicted enhancement factors $\tilde{b}_{\max }(n) / \tilde{b}_{\max }(0)$ as obtained from Eq. (4.23).

between the average velocity and the driving force when comparing Figs. 4.5 $(a)$ and 4.5(b): $u_{a v}$ is not proportional to $\mathrm{d} p / \mathrm{d} x$. Because of the shear-thinning properties of the fluid, the flow rate increases faster than the applied pressure gradient. 
The results described in this section underline that it is important that the constitutive equation properly describes the rheological behaviour of the fluid for very small or very large shear rates. A constitutive equation like the Carreau expression does this; modelling the liquid as a power-law fluid would have predicted an enhancement in slip for all flow/shear rates [58]. However, when operating in the shear-thinning regime with shear rates in the range of roughly $10^{-1}<\mathrm{d} u / \mathrm{d} y<10^{3}$ $1 / \mathrm{s}$, the power-law does predict the correct behaviour.

\subsubsection{Predicting the enhancement}

In Sec. 4.3 we have derived an expression that gives the maximum slip length that can be obtained when the bubbles become infinitely large compared to the typical system size, i.e. when $\tilde{L}=L / R \rightarrow \infty$. The results presented above, however, are for a bubble mattress with $H=100 \mu \mathrm{m}, L_{g}=10 \mu \mathrm{m}$ and $\varepsilon=1 / 2$. This gives only a confinement factor of $L / H=0.2$.

To investigate how the amount of wall slip changes with increasing confinement, we have simulated fluid flow through a tube with a pattern of transverse no-slip and no-shear regions. This can be interpreted as a cylindrical bubble mattress with flat bubbles $\left(\vartheta=0^{\circ}\right)$. The surface ratio of no-slip to no-shear region equals $\varepsilon=1 / 2$. The results are plotted in Fig. 4.6(a). For $\tilde{L}<10^{0}$, where the slope of the log-log plot equals one, the effective slip length $\tilde{b}$ grows linearly with the confinement $\tilde{L}$. As will be discussed in Sec. 4.4.3, for $\tilde{L}<10^{0}$ the flow profile cannot fully adapt to either the no-slip or no-shear condition at the wall and is therefore still hydrodynamically developing. The slip length reaches its maximum when $\tilde{L}>10^{2}$, showing a perfect overlap with the analytically calculated values given by Eq. (4.23).

Note that in Fig. 4.6(a) the slip length is non-dimensionalised using the tube radius $R$, whereas in Figs. 4.4 and 4.5 the slip length is non-dimensionalised based on the bubble width $L_{g}$. These are related to each other, as

$$
\frac{2 b}{L_{g}}=\frac{2 \tilde{b}}{\varepsilon \tilde{L}} .
$$

This implies that plotting $2 b / L_{g}(\tilde{L})$ instead of $\tilde{b}(\tilde{L})$ will reduce the slope of the profiles in Fig. 4.6(a) by one. Fig. 4.6(b) shows that this indeed is the case. For $\tilde{L}<10^{0}, 2 b / L_{g}$ is constant, whereas this decreases linearly for $\tilde{L}>10^{2}$.

Two limiting regimes can therefore be identified. In the first regime, the bubble confinement is very small $(\tilde{L} \ll 1)$ and $2 b / L g$ is constant. The slip length is 'suboptimal' in the sense that it is smaller than the maximum slip possible for the set of wall conditions. The slip length grows linearly with the length of the no-shear region $\varepsilon \tilde{L}$, which can be interpreted as the bubble width. In the second regime, the bubble confinement is very large $(\tilde{L} \gg 1)$ and $\tilde{b}$ is constant. The 
(a) $\varepsilon=0.5$

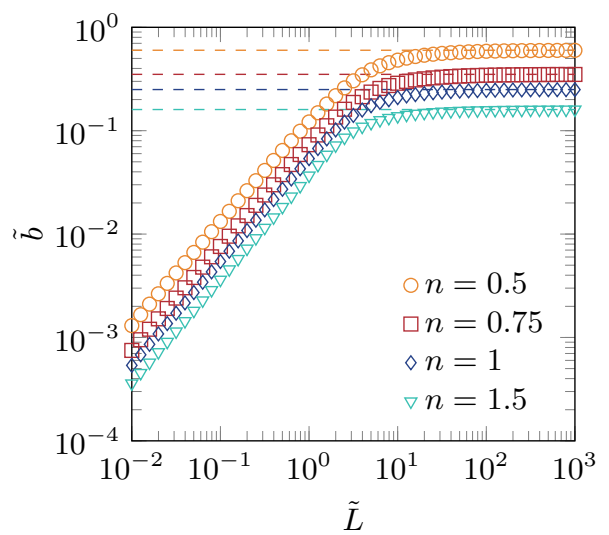

(c) $\varepsilon=0.5$

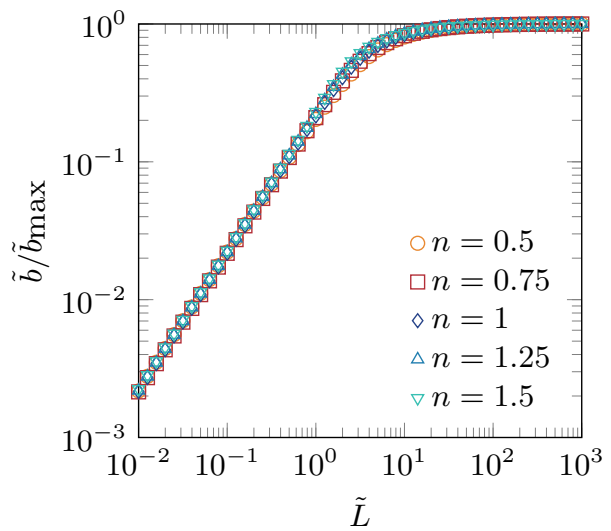

(b) $\varepsilon=0.5$

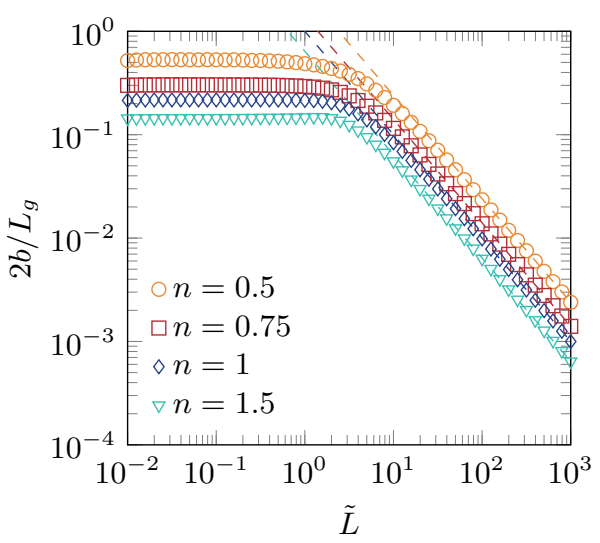

(d) $\varepsilon=0.5$

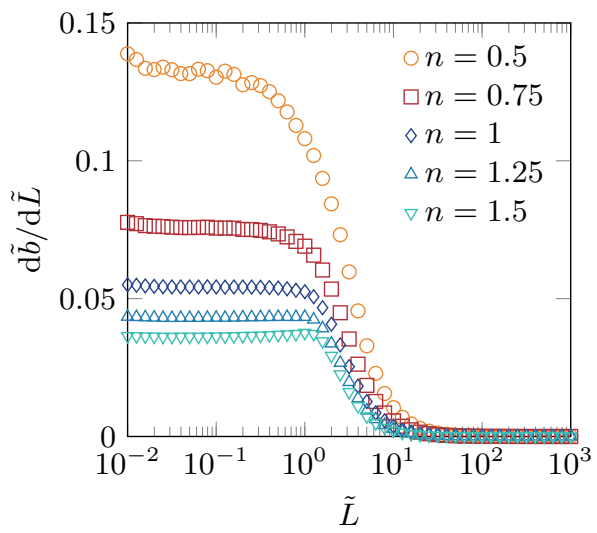

Figure 4.6 - In $(a)$ the effective slip length $\tilde{b}=b / R$ is plotted as a function of the no-slip/no-shear unit length $\tilde{L}=L / R$ for various power-law indices $n$. The same data is replotted in $(b)$, showing now the slip length normalised with the length of the no-shear region $L_{g}$. The dashed lines in $(a)$ and $(b)$ are the theoretical maxima for $\tilde{b}$ as calculated from Eq. (4.23). When normalising $\tilde{b}$ using the maximum effective slip length $\tilde{b}_{\max }$, the master curve shown in $(c)$ is obtained. In $(d)$ the slope $\mathrm{d} \tilde{b} / \mathrm{d} \tilde{L}$ of the slip length profiles shown in $(a)$ are plotted.

slip length reaches its maximum value, i.e. $\tilde{b}=\tilde{b}_{\max }$, and the effective pressure gradient is constant, because it is fully dominated by the pressure drop over the no-slip region, which grows linearly with the length $(1-\varepsilon) \tilde{L}$ of this region.

Fig. 4.6(c) illustrates that the expression for maximum wall slip, Eq. (4.23), can also be used to predict the enhancement in the regime $\tilde{L}<10^{0}$ where $2 b / L_{g}$ is constant. All profiles for $\tilde{b}$ collapse onto a master curve when normalising them by the corresponding maximum slip length $\tilde{b}_{\max }$. This indicates that the ratio in 


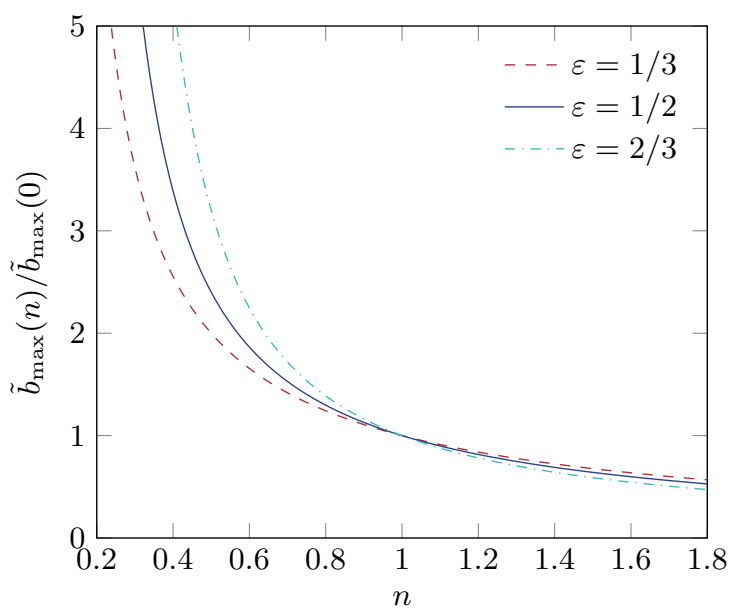

Figure 4.7 - Wall slip enhancement factor $\tilde{b}_{\max }(n) / \tilde{b}_{\max }(0)$, given by Eq. (4.23), as a function of the power-law index $n$ for three different surface porosities $\varepsilon$.

$\tilde{b}_{\text {max }}$ for power-law fluids having a different exponents $n$ remains constant for all $\tilde{L}$, except for the transition regime $10^{0}<\tilde{L}<10^{2}$.

The slope of the profiles must be different for varying power-law indices $n$, since $\tilde{b} \rightarrow 0$ when $\tilde{L} \rightarrow 0$. The effective slip length needs to grow faster for a shear-thinning than for a shear-thickening liquid, as all power-law fluids reach $\tilde{b}_{\max }$ for approximately the same value of $\tilde{L}$. Fig. $4.6(d)$ supports this conclusion. Note that the slope of the curves not only changes with $n$, but also with the surface porosity $\varepsilon$. We found that the slope scales approximately as $\mathrm{d} \tilde{b} / \mathrm{d} \tilde{L} \propto 1 / n$ and $\mathrm{d} \tilde{b} / \mathrm{d} \tilde{L} \propto \varepsilon /(1-\varepsilon)$.

The maximum slip length $\tilde{b}_{\text {max }}$ predicted by Eq. (4.23) is plotted in Fig. 4.7 as function of the power-law index $n$ for three different surface porosities $\varepsilon$. The curves, which have been normalised by $\tilde{b}_{\max }$ for a Newtonian fluid with $n=1$, give the slip enhancement factor for a given $n$ and $\varepsilon$. Shear-thinning behaviour $(n<1)$ increases relative wall slip, whereas shear thickening behaviour $(n>1)$ reduces it. Wall slip increases with $\varepsilon$ for shear-thinning liquids with $n<1$, whereas it decreases with $\varepsilon$ for shear-thickening fluids with $n>1$.

We found that Eq. (4.23) can also be used to predict the maximum slip length in the regime $\tilde{L}<10^{0}$ where $\left(2 b / L_{g}\right)$ is constant. For that reason, we plotted in Fig. $4.5(b)$ the analytically predicted enhancement factors for the three different xanthan gum solutions. The horizontal dashed lines in Fig. 4.5(b), which represent the analytical values, indeed show very good agreement with the numerically calculated maximum enhancement factors. 
$(a)$

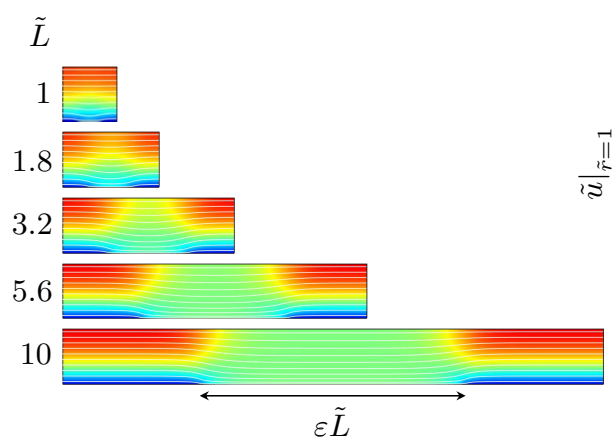

(b) $n=1$, various $\tilde{L}, \varepsilon=1 / 2$

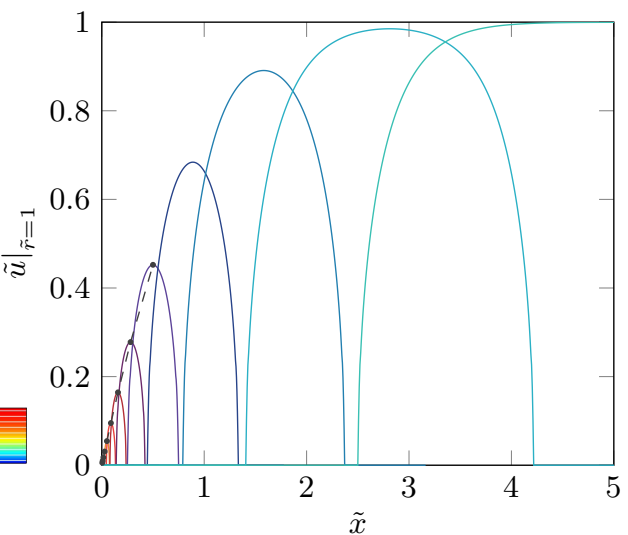

Figure 4.8 - As long as the flow is hydrodynamically developing, the slip length $\tilde{b}$ grows linearly with the unit length $\tilde{L}$. (a) For $\varepsilon \tilde{L}>2.4$, the flow becomes hydrodynamically developed. (b) The liquid velocity at the no-shear wall then also reaches the maximum possible value of $\left.\tilde{u}\right|_{\tilde{r}=1}=1$. Here, the velocity profiles are plotted for $\tilde{L}=10^{-2+0.25 k}$ with $k=[0 \ldots 12]$. The increase in the maximum liquid velocity at the wall with $\tilde{L}$ for different power law indices $n$ (here represented for $n=1$ by the grey dashed curve for $\tilde{L} \leq 1)$ is correlated with $\mathrm{d} \tilde{b} / \mathrm{d} \tilde{L}$ as plotted in Fig. $4.6(d)$, showing the same behaviour.

\subsubsection{Relationship to hydrodynamic development length}

Fig. 4.6(a) shows that for $\tilde{L}<10^{0}$, the effective slip length $\tilde{b}$ grows linearly with $\tilde{L}$. This can be related to the hydrodynamic development of the velocity profile. The length $L_{d}$ required for full development, also called the entrance length, is given by [59]

$$
\frac{L_{d}}{D}=0.6+0.056 R e
$$

which in case of Stokes flow with $R e \rightarrow 0$ and using the radius $R$ instead of the diameter $D$ reduces to

$$
\frac{L_{d}}{R}=1.2
$$

Considering the flow over the no-slip and no-shear regions, illustrated in Fig. 4.8(a), we observe that in each region the flow is developing twice: when entering and when exiting the region. Therefore we expect the profile to be fully developed when

$$
\begin{cases}\varepsilon \tilde{L}>2.4 & \text { when } \varepsilon \leq 1 / 2, \\ (1-\varepsilon) \tilde{L}>2.4 & \text { when } \varepsilon>1 / 2 .\end{cases}
$$

This is an estimation, as in the model the flow is already partly developed when entering the region. Eq. (4.26) is based on a system where at the start of a 
no-slip region the velocity profile is uniform (which, in the case of Stokes flow as considered here, is equivalent to a system with a parabolic velocity profile at the start of a no-shear region). Note that the entrance length only changes slightly for an inelastic non-Newtonian fluid [60].

The development for a Newtonian liquid, i.e. $n=1$, is also illustrated in Fig. $4.8(a)$. When $\tilde{L}=5.6$, we see that the profile in the middle of the domain is uniform. This is also confirmed in Fig. 4.8(b), which shows that for this domain length the velocity at the wall essentially reaches its maximum possible value: $\left.\tilde{u}\right|_{\tilde{r}=1}=1$. The results shown in Fig. 4.8 are therefore in agreement with Eq. (4.28), which predicts that for this system the flow is hydrodynamically developed when $\tilde{L}>4.8$.

The slip length $\tilde{b}$ (Fig. 4.6(a)) does not increase linearly with $\tilde{L}$ anymore when $\varepsilon \tilde{L}>2.4$. It requires $\tilde{L}$ to be roughly two orders of magnitude larger, however, to let the slip length reach the actual maximum $\tilde{b}_{\max }$. In the transition region $10^{0}<\tilde{L}<10^{2}$ the development regions $\tilde{l}_{1}$ and $\tilde{l}_{3}$ (see Fig. 4.8) still contribute to the overall or effective pressure gradient over the system. This gradient slowly converges to the pressure drop over the non-slippery region, wherefore ultimately the conditions are reached for which the expression for $\tilde{b}_{\max }$, Eq. (4.23), is valid.

\subsection{Conclusion}

In this study, we have investigated the flow of inelastic, non-Newtonian fluids over heterogeneously slippery superhydrophobic surfaces. The numerical and analytical results show that wall slip is increased for shear-thinning liquids, whereas shear-thickening fluids result in reduced slippage. In the case of shear-thinning liquids, for which we took aqueous xanthan gum solutions as model fluids, the slip enhancement with respect to water strongly depends on the applied driving force. Only in the shear-thinning regime, wall slip can be enhanced. The slip length converges to that of water when the system is operated in one of the Newtonian regimes.

The maximum slip enhancement could be predicted analytically for a cylindrical geometry. The predicted enhancement showed good agreement with the numerical results for a planar geometry. This indicates that the theoretical result is also applicable to other geometries. Wall slip is maximised when the bubbles are much larger than the characteristic dimension of the system: the flow over the heterogeneous surface can then fully develop according to the hydrodynamic boundary conditions. Although in practice it is hard to achieve such conditions (the slip regions are highly confined), the enhancement factor as derived analytically from the maximum slip length remains constant for all confinement factors.

Experiments are required to confirm the predicted increase in wall slip for 
flow of inelastic shear-thinning liquids over heterogeneously slippery surfaces. Aqueous xanthan gum solutions appear to be suitable for this purpose, since the preliminary experimental results indicate that for these solutions slip induced by depletion effects at the wall is either absent or negligible compared to slip arising from superhydrophobicity. The experiments are challenging in terms of stability and controllability, as stable non-Newtonian fluid flows over microscale, heterogeneously slippery surfaces need to be established at low flow rates. However, they are of high value to explore the potential of superhydrophobic slip enhancement for non-Newtonian fluid flow.

\section{References}

[1] C. L. M. H. Navier, Mémoire sur les lois du mouvement des fluids, Mem. Acad. Sci. Int. Fr. 6, 389 (1823).

[2] C. Neto, D. R. Evans, E. Bonaccurso, H.-J. Butt, and V. S. J. Craig, Boundary slip in Newtonian liquids: a review of experimental studies, Rep. Prog. Phys. 68, 2859 (2005).

[3] J. P. Rothstein, Slip on superhydrophobic surfaces, Annu. Rev. Fluid Mech. 42, 89 (2010).

[4] T. Lee, E. Charrault, and C. Neto, Interfacial slip on rough, patterned and soft surfaces: a review of experiments and simulations, Adv. Colloid Interfac. 210, 21 (2014).

[5] R. S. Voronov, D. V. Papavassiliou, and L. L. Lee, Review of fluid slip over superhydrophobic surfaces and its dependence on the contact angle, Ind. Eng. Chem. Res. 47, 2455 (2008).

[6] Y. Xue, P. Lv, H. Lin, and H. Duan, Underwater superhydrophobicity: stability, design and regulation, and applications, Appl. Mech. Rev. 68, 030803 (2016).

[7] Y. Cohen and A. B. Metzner, Apparent slip flow of polymer solutions, J. Rheol. 29, 67 (1985).

[8] W. Schowalter, The behavior of complex fluids at solid boundaries, J. NonNewt. Fluid Mech. 29, 25 (1988).

[9] H. A. Barnes, A review of the slip (wall depletion) of polymer solutions, emulsions and particle suspensions in viscometers: its cause, character, and cure, J. Non-Newt. Fluid Mech. 56, 221 (1995).

[10] T. Sochi, Slip at fluid-solid interface, Polym. Rev. 51, 309 (2011).

[11] S. G. Hatzikiriakos, Slip mechanisms in complex fluid flows, Soft Matter 11, 7851 (2015).

[12] F. Brochard and P. G. de Gennes, Shear-dependent slippage at a polymer/solid interface, Langmuir 8, 3033 (1992). 
[13] K. B. Migler, H. Hervet, and L. Leger, Slip transition of a polymer melt under shear-stress, Phys. Rev. Lett. 70, 287 (1993).

[14] A. L. Yarin, A model for slip at polymer/solid interfaces, J. Rheol. 42, 1491 (1998).

[15] H. C. Lau and W. R. Schowalter, A model for adhesive failure of viscoelastic fluids during flow, J. Rheol. 30, 193 (1986).

[16] S. Richardson, On the no-slip boundary condition, J. Fluid Mech. 59, 707 (1973).

[17] Y. Zhu and S. Granick, Limits of the hydrodynamic no-slip boundary condition, Phys. Rev. Lett. 88, 106102 (2002).

[18] S. Granick, Y. Zhu, and H. Lee, Slippery questions about complex fluids flowing past solids, Nat. Mater. 2, 221 (2003).

[19] J. Sanchez-Reyes and L. A. Archer, Interfacial slip violations in polymer solutions: role of microscale surface roughness, Langmuir 19, 3304 (2003).

[20] A. Y. Malkin, The state of the art in the rheology of polymers: achievements and challenges, Polym. Sci. Ser. A 51, 80 (2009).

[21] R. Tuinier and T. Taniguchi, Polymer depletion-induced slip near an interface, J. Phys. Cond. Mat. 17, L9 (2005).

[22] D. Ausserré, H. Hervet, and F. Rondelez, Concentration dependence of the interfacial depletion layer thickness for polymer solutions in contact with nonadsorbing walls, Macromolecules 19, 85 (1986).

[23] H. Müller-Mohnssen, Concentration dependent changes of apparent slip in polymer solution flow, J. Rheol. 34, 223 (1990).

[24] A. Omari, Hydrodynamic behavior of semirigid polymer at a solid-liquid interface, J. Rheol. 33, 1 (1989).

[25] E. Duering and Y. Rabin, Polymers in shear flow near repulsive boundaries, Macromolecules 23, 2232 (1990).

[26] D. Ausserré, J. Edwards, J. Lecourtier, H. Hervet, and F. Rondelez, Hydrodynamic thickening of depletion layers in colloidal solutions, Europhys. Lett. 14, 33 (1991).

[27] G. Chauveteau, Rodlike polymer solution flow through fine pores: influence of pore size on rheological behavior, J. Rheol. 26, 111 (1982).

[28] A. M. Jamieson, J. G. Southwick, and J. Blackwell, Dynamical behavior of xanthan polysaccharide in solution, J. Polym. Sci. A2 Polym. Phys. 20, 1513 (1982).

[29] M. Milas, M. Rinaudo, and B. Tinland, The viscosity dependence on concentration, molecular weight and shear rate of xanthan solutions, Polym. Bull. 14, 157 (1985).

[30] G. Cuvelier and B. Launay, Concentration regimes in xanthan gum solutions deduced from flow and viscoelastic properties, Carbohyd. Polym. 6, 321 (1986). 
[31] M. Milas, M. Rinaudo, M. Knipper, and J. L. Schuppiser, Flow and viscoelastic properties of xanthan gum solutions, Macromolecules 23, 2506 (1990).

[32] A. Gamini and M. Mandel, Physicochemical properties of aqueous xanthan solutions: static light scattering, Biopolymers 34, 783 (1994).

[33] S. K. Gulrez, S. Al-Assaf, Y. Fang, G. O. Phillips, and A. P. Gunning, Revisiting the conformation of xanthan and the effect of industrially relevant treatments, Carbohyd. Polym. 90, 1235 (2012).

[34] J. R. Philip, Flows satisfying mixed no-slip and no-shear conditions, Z. angew. Math. Phy. 23, 353 (1972).

[35] E. Lauga and H. A. Stone, Effective slip in pressure-driven Stokes flow, J. Fluid Mech. 489, 55 (2003).

[36] C. Ybert, C. Barentin, C. Cottin-Bizonne, P. Joseph, and L. Bocquet, Achieving large slip with superhydrophobic surfaces: scaling laws for generic geometries, Phys. Fluids 19, 123601 (2007).

[37] S. J. Gibbs, D. Xing, T. A. Carpenter, and L. D. Hall, NMR flow imaging of aqueous polysaccharide solutions, J. Rheol. 38, 1757 (1994).

[38] S. J. Gibbs, Rheometry and detection of apparent wall slip for Poiseuille flow of polymer solutions and particulate dispersions by nuclear magnetic resonance velocimetry, J. Rheol. 40, 425 (1996).

[39] C. J. Rofe, Nuclear magnetic resonance imaging of apparent slip effects in xanthan solutions, J. Rheol. 40, 1115 (1996).

[40] J. Davies, D. Maynes, B. W. Webb, and B. Woolford, Laminar flow in a microchannel with superhydrophobic walls exhibiting transverse ribs, Phys. Fluids 18, 087110 (2006).

[41] D. Maynes, K. Jeffs, B. Woolford, and B. W. Webb, Laminar flow in a microchannel with hydrophobic surface patterned microribs oriented parallel to the flow direction, Phys. Fluids 19, 093603 (2007).

[42] E. S. Asmolov, S. Schmieschek, J. Harting, and O. I. Vinogradova, Flow past superhydrophobic surfaces with cosine variation in local slip length, Phys. Rev. E 87, 023005 (2013).

[43] C. Schönecker and S. Hardt, Longitudinal and transverse flow over a cavity containing a second immiscible fluid, J. Fluid Mech. 717, 376 (2013).

[44] C. J. Teo and B. C. Khoo, Effects of interface curvature on Poiseuille flow through microchannels and microtubes containing superhydrophobic surfaces with transverse grooves and ribs, Microfluid. Nanofluid. 17, 891 (2014).

[45] G. G. Pereira, Effect of variable slip boundary conditions on flows of pressure driven non-Newtonian fluids, J. Non-Newt. Fluid Mech. 157, 197 (2009).

[46] S. Dhondi, G. G. Pereira, and S. C. Hendy, Molecular dynamics simulations of polymeric fluids in narrow channels: methods to enhance mixing, Phys. Rev. E 80, 036309 (2009).

[47] A.-L. Vayssade, C. Lee, E. Terriac, F. Monti, M. Cloitre, and P. Tabeling, 
Dynamical role of slip heterogeneities in confined flows, Phys. Rev. E 89, 052309 (2014).

[48] D. Broboana, N. O. Tanase, and C. Balan, Influence of patterned surface in the rheometry of simple and complex fluids, J. Non-Newt. Fluid Mech. 222, 151 (2015).

[49] P. Nghe, E. Terriac, M. Schneider, Z. Li, M. Cloitre, B. Abecassis, and P. Tabeling, Microfluidics and complex fluids, Lab Chip 11, 788 (2011).

[50] F. J. Galindo-Rosales, M. A. Alves, and M. S. N. Oliveira, Microdevices for extensional rheometry of low viscosity elastic liquids: a review, Microfluid. Nanofluid. 14, 1 (2013).

[51] P. Yager, T. Edwards, E. Fu, K. Helton, K. Nelson, M. R. Tam, and B. H. Weigl, Microfluidic diagnostic technologies for global public health, Nature 442, 412 (2006).

[52] M. Karle, S. K. Vashist, R. Zengerle, and F. von Stetten, Microfluidic solutions enabling continuous processing and monitoring of biological samples: a review, Anal. Chim. Acta 929, 1 (2016).

[53] H. A. Barnes, J. F. Hutton, and K. Walters, An introduction to rheology (Elsevier, Amsterdam, 1989).

[54] P. E. Arratia, G. A. Voth, and J. P. Gollub, Stretching and mixing of non-Newtonian fluids in time-periodic flows, Phys. Fluids 17, 1 (2005).

[55] A. M. J. Davis and E. Lauga, Geometric transition in friction for flow over a bubble mattress, Phys. Fluids 21, 011701 (2009).

[56] E. Karatay, A. S. Haase, C. W. Visser, C. Sun, D. Lohse, P. A. Tsai, and R. G. H. Lammertink, Control of slippage with tunable bubble mattresses., P. Natl Acad. Sci. USA 110, 8422 (2013).

[57] T. F. Assis, E. E. G. Rojas, G. C. Guimarães, M. C. Coelho, A. V. Ramos, B. S. Costa, and J. S. R. Coimbra, Kinematic viscosity and density of binary and ternary mixtures containing hydrocolloids, sodium chloride, and water, Int. J. Thermophys. 31, 513 (2010).

[58] L. M. J. Sprakel, Non-Newtonian flow on bubble mattresses, MSc thesis, University of Twente, 2014.

[59] J. A. Schetz and A. E. Fuhs, Fundamentals of fluid mechanics (John Wiley \& Sons, New York, 1999).

[60] R. J. Poole and B. S. Ridley, Development-length requirements for fully developed laminar pipe flow of inelastic non-Newtonian liquids, J. Fluids Eng. 129, 1281 (2007). 


\section{Appendix}

Experimentally, wall slip for non-Newtonian fluid flow was investigated using micro-particle image velocimetry $(\mu \mathrm{PIV})$ [58]. For this purpose, glass-silicon microfluidic devices were fabricated using standard photolitography and deep reactive ion etching. The structured silicon wafers were anodically bonded to glass wafers. The silicon microchannels, which were all $100 \mu \mathrm{m}$ deep, had different main channel heights $H$, side channel widths $L_{g}$ and surface porosities $\varepsilon$ (see Fig. 4.9(a) for an example). The channels were hydrophobised using gaseous chlorotrimethylsilane (Sigma-Aldrich).

For the $\mu \mathrm{PIV}$ experiments, a Litron LDY301 dual cavity Nd:YLF laser with a wavelength of $527 \mathrm{~nm}$ connected to an inverted Zeiss Axio Observer.Z1 microscope was used. The microscope was equipped with a LaVision Imager intense 670KD double shutter camera. The experiments were performed under stationary flow with an average liquid velocity of $u_{a v}=10^{-1} \mathrm{~m} / \mathrm{s}$ using a Bronkhorst mass flow controller (see Fig. 4.9(b)). A Bronkhorst pressure controller was used to regulate the gas pressure. Various xanthan gum (G1253, Sigma-Aldrich) solutions in a mixture of glycerol (Sigma-Aldrich) and water were used, in which 0.01 wt\% red fluorescing polystyrene particles (microParticles, Germany) with a diameter of $0.5 \mu \mathrm{m}$ were dispersed. To determine the flow field, required to evaluate the effective slip length, 100 image pairs were captured at a $63 \times$ magnification (with a numerical aperture of 0.75 ). The time difference between frame $\mathrm{A}$ and $\mathrm{B}$ of each image pair was set to $6 \mu \mathrm{s}$, which gave an optimal average particle shift of about 5 pixels. The images were preprocessed by removal of the background intensity and the out-of-focus particles. The occurrence of spurious vectors in the vector fields was suppressed by using a geometric mask. The vector fields were obtained using the DaVis 8 PIV software package by a multi-pass calculation, starting with two passes using an interrogation window of $64 \times 64$ pixels and finishing with an interrogation window of $16 \times 16$ pixels, all with a $50 \%$ window overlap. A sliding sum of correlation was employed, which uses five consecutive image pairs for the cross correlation to artificially increase the particle density.

The local slip lengths were determined by extrapolation of the local velocity profile using the six data points adjacent to the wall. Averaging of these local slip lengths gave the effective slip length.

The experimentally determined effective slip lengths are plotted in Fig. 4.9(c) for a chip with $H=50 \mu \mathrm{m}, L_{g}=10 \mu \mathrm{m}$ and $\varepsilon=2 / 3$ for various liquids. The slip lengths all show the same behaviour: decreasing wall slip with increasing protrusion angle $\vartheta$. Additionally, the slip lengths are quantitatively similar for all liquids. This is as expected: because of the high flow rate $\left(u_{a v}=10^{-1} \mathrm{~m} / \mathrm{s}\right)$, in a channel with a height of $H=50 \mu \mathrm{m}$ the shear rates are of the order of $\mathrm{d} u / \mathrm{d} y=10^{3} 1 / \mathrm{s}$. According to the flow curves given in Fig. 4.1, the system is 

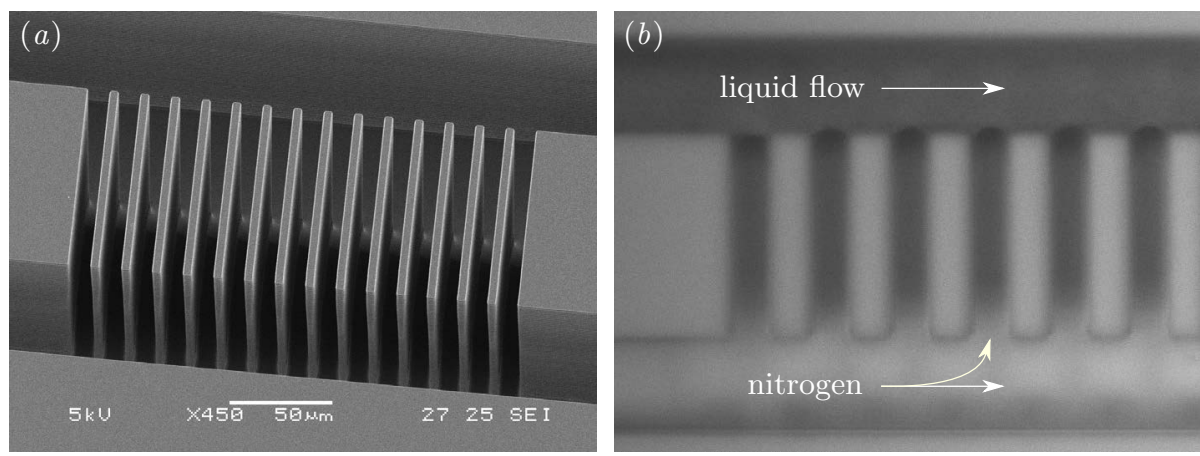

(c) $H=50 \mu \mathrm{m}, L_{g}=10 \mu \mathrm{m}$ and $\varepsilon=2 / 3$

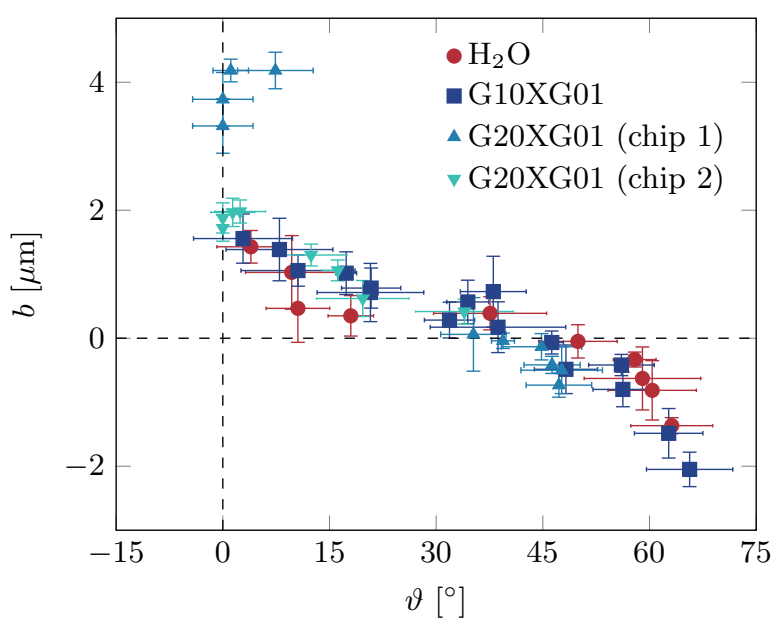

Figure 4.9 - In (a) a SEM image is shown of one of the microfluidic devices that were used to study non-Newtonian flow over a bubble mattress. They consist of two main channels, connected to each other by 15 microchannels. Because the wall was hydrophobised, as shown in $(b)$ the side channels were filled with a gas (nitrogen) and the liquid in the upper channel was flowing over an array of no-slip wall segments and gas bubbles. Here, the image was focussed at the midplane of the channel, where also the $\mu \mathrm{PIV}$ experiments were performed. In $(c)$, the effective slip lengths $b$ are plotted as a function of the bubble protrusion angle $\vartheta$ for water and for shear-thinning solutions of $0.1 \mathrm{wt} \%$ xanthan gum (XG) dissolved in a mixture of water and either 10 or $20 \mathrm{wt} \%$ glycerol (G).

thus operated in the upper Newtonian regime and the viscosity of the xanthan gum solutions approaches the value for infinite shear. These results indicate that for aqueous xanthan gum solutions depletion-induced wall slip is either absent or negligible compared to slip arising from superhydrophobicity. 



\title{
CHAPTER 5
}

\section{Momentum and mass transport over a bubble mattress: the influence of interface geometry}

\begin{abstract}
In this study we investigate numerically how a partially slippery bubble mattress can be employed to enhance momentum and mass transport. The bubble mattress, which is a superhydrophobic surface, consists of an array of slippery gas bubbles with no-slip walls in between. We consider pressure-driven laminar flow over the bubble mattress, with a solute being supplied from the gas bubbles. The results show that, because of effective wall slip, solute transport can be enhanced significantly with respect to a fully saturated no-slip wall. The enhancement depends on the interface geometry of the bubble mattress, i.e. on the bubble size, protrusion angle and surface porosity. In addition, we demonstrate that the mass transfer enhancement disappears below a critical bubble size. The effective slip vanishes for very small bubbles, wherefore interfacial transport becomes diffusion dominated. For large bubbles, solute transport near the interface is convection enhanced.
\end{abstract}

This chapter has been published as A.S. Haase, E. Karatay, P.A. Tsai and R.G.H. Lammertink, Momentum and mass transport over a bubble mattress: the influence of interface geometry, Soft Matter 9, 8949 (2013). 


\subsection{Introduction}

Interfacial transport phenomena can be enhanced considerably by employing superhydrophobic surfaces that are characterised by (partial) wall slip [1-3]. It has been shown theoretically that, even with slip lengths of the order of nanometres, interfacially driven transport of for instance mass and heat can be enhanced up to two orders of magnitude [4]. Various studies have been performed that address the influence of wall slip on interfacial transport in electro-osmotic flows [4-7], in thin film evaporation in microchannels [8], and in heat and mass transfer through permeable surfaces [9-13]. It is found that concentration polarisation at membrane walls decreases with increasing slip, which results in higher interfacial mass transfer rates $[10,14-16]$. In general, all these studies report a significant amplification of the transport process under consideration in the presence of a slippery interface. In this study, we investigate the effect of the interface geometry on both momentum and mass transport over a partially slippery bubble mattress using the two-dimensional model shown in Fig. 5.1( $a)$, where the no-shear regions are positioned perpendicular to the direction of a pressure-driven laminar flow.

Wall slip can be quantified by a slip length $b$, which can be interpreted as the imaginary distance beyond the surface where the no-slip boundary condition (BC) would be satisfied $[17,18]$. In a two-dimensional system, where $y$ is the axis perpendicular to the slip surface, $u$ is the bulk liquid velocity in the $x$-direction, and $u_{b}$ is the liquid velocity at the slippery surface, the slip length $b$ is commonly defined by Navier's slip condition [19]:

$$
u_{b}=\left.b \frac{\partial u}{\partial y}\right|_{y=0}
$$

For smooth hydrophobic surfaces typical slip lengths are in the nanometre range $[20,21]$. This is too small to realise a significant reduction in wall friction. However, slippage can be increased significantly by micro-structuring the hydrophobic surface with posts, grooves or cavities that are positioned transversely, longitudinally or obliquely to the main flow direction [13, 22-30]. As gas is entrapped in these microstructures, the liquid is in contact with a heterogeneous gas-solid interface, partially characterised by slip conditions. Measured apparent or effective slip lengths on such superhydrophobic surfaces range from $10^{0}$ to $10^{2} \mu \mathrm{m}[21,24,29,31-33]$.

Recent numerical studies have shown that for bubble mattress-like geometries the effective slip is a function of the interface geometry, i.e. the extent of bubble protrusion in the liquid, the bubble shape, the bubble size and the porosity of the surface [27, 28, 34-36]. This is in agreement with multiple analytical studies $[18,21,37-44]$. The analytical model of Davis and Lauga [18], which considers two-dimensional shear flow over an array of bubbles with size $L_{g}$, see Fig. 5.1(b), 
predicts that for a given bubble protrusion angle $\vartheta$ the dimensionless slip length $2 b / L_{g}$ is proportional to the surface porosity $\varepsilon=L_{g} / L$ :

$$
\frac{2 b}{L_{g}}=\pi \varepsilon \int_{0}^{\infty} A(s) \mathrm{d} s,
$$

where

$$
\begin{aligned}
A(s) & =\frac{s}{\sinh 2 s(\pi-\vartheta)+s \sin 2 \vartheta} \times \\
& {\left[\cos 2 \vartheta+\frac{s \sinh 2 \vartheta \cosh s \pi+\sinh s(\pi-2 \vartheta)}{\sinh s \pi}\right] . }
\end{aligned}
$$

Evaluation of this model shows that wall slip is positive for negative $\vartheta$ and positive $\vartheta$ up to a critical protrusion angle $\vartheta_{c}$. Maximum slip lengths are obtained for relatively flat bubbles. For $\vartheta>\vartheta_{c}$ negative slip lengths have been reported due to obstruction of the flow by the bubbles that protrude deeply in the liquid flow $[18,27,34,36]$.

Interfacial solute transport across the bubble mattress is directly coupled to momentum transport over the partially slippery surface. The effects of surface porosity and interpore spacing on mass transfer through heterogeneous surfaces such as membranes are known for a longer time [45-48]. Juhasz and Deen [47, 48] investigated mass transfer for a heterogeneous surface where at the non-slippery active sites a non-zero solute concentration was applied, with a convective flow parallel to the surface. They found that as a result of convection, concentration variations along the heterogeneous surface are minimised, thereby augmenting mass transfer. Furthermore, they showed that the mass transfer rate approaches the theoretical limit defined by a fully saturated wall when the bubble size becomes very small or when the liquid velocity is very large. Mass transfer appears to be proportional to the surface porosity in the case of large bubbles and moderate fluid flows.

This provides an explanation why Kreulen et al. [49] did not find any influence of the surface porosity on the ultimate solute flux for a hollow fibre membrane. The fluxes they measured for fibres with a porosity of $3 \%$ and $70 \%$ all fit to the analytical expression for a fully saturated no-slip wall. They argue that the fibre wall is solute-satured because of the extremely small distance between the pores compared to the distance to the centre of the fibre. Consequently, they conclude that for determination of the mass transfer coefficient the total membrane area has to be used. This conclusion supports the commonly used assumption in membrane technology that a microporous membrane wall is both solute-saturated and non-slippery [49-51].

Both Juhasz and Deen, and Kreulen et al. presuppose that the heterogeneous surface is flat and non-slippery. This explains why the mass transfer limit is given 
by a fully saturated no-slip wall. In this numerical study, however, the influence of the interface geometry of a partially slippery bubble mattress on both momentum and mass transport is systematically investigated by varying the bubble protrusion angle, surface porosity and bubble size. We show that, as a result of effective wall slip, mass transfer with respect to a solute-saturated no-slip wall can be enhanced significantly. In addition, we provide a theoretical analysis that explains why for very small no-shear bubbles the enhancement vanishes. The estimated critical bubble size at which diffusive transport equals convective transport, as obtained from the local Péclet number, proves to be in good agreement with the numerically found bubble sizes for which solute transport is no longer significantly enhanced.

\subsection{Mathematical formulation}

\subsubsection{Computational model}

The computational model of the bubble mattress is schematically shown in Fig. 5.1(b). The model is a two-dimensional simplification of our previously reported microfluidic devices that were used for experimental investigation of the influence of the interface geometry on effective slip [33]. In these devices, a bubble mattress was established by supplying a gas through small side channels positioned perpendicular to the main liquid channel.

The non-periodic numerical model considers two-dimensional steady laminar liquid flow in a channel. The liquid flow is treated as incompressible, with a constant liquid viscosity. It is assumed that the gas phase has negligible viscosity with respect to the liquid viscosity. As such a no-shear boundary condition is applied to the bubble surface (i.e. $b=\infty$ ). In addition, a constant solute concentration is imposed on the gas-liquid interface. In all simulations the capillary number is $C a=\mu L_{g} \gamma / \sigma \leq 10^{-4}$, where $\mu$ is the liquid viscosity, $\gamma$ is the local shear rate and $\sigma$ is the surface tension. Accordingly the bubble surface can assumed to be rigid and spherical $[28,34]$.

The fluid is flowing in a channel of height $H=100 \mu \mathrm{m}$, with an upper no-slip wall and a bubble mattress as the lower wall. The fluid flow in the $x$-direction is pressure-driven by a fixed pressure gradient $\Delta p / \Delta x=-1800 \mathrm{~Pa} / \mathrm{m}$ over the computational domain, where $\Delta x=680 \mu \mathrm{m}$. The bubble mattress consists in total of $N$ bubble units with a total length of $N L=600 \mu \mathrm{m}$. To ensure fluid flow is developed at the beginning of the bubble mattress, it is preceded by an entrance domain of $40 \mu \mathrm{m}$ length. Correspondingly, an outlet domain of $40 \mu \mathrm{m}$ length is added to the geometry.

We vary the interface geometry by changing the bubble protrusion angle $\vartheta$, the surface porosity $\varepsilon$ and the bubble size $L_{g}$. When changing $\varepsilon=L_{g} / L$, the 
bubble size $L_{g}=L-L_{s}$ is kept constant. Here $L_{s}$ is the size of the solid wall in between the bubbles (see Fig. 5.1(b)). The surface porosity is either $\varepsilon=1 / 3$, or $\varepsilon=1 / 2$ or $\varepsilon=2 / 3$. In each simulation, the protrusion angle is varied between $-90^{\circ}<\vartheta<90^{\circ}$. To investigate the effect of bubble size on effective slip, simulations have been performed for $L_{g}=1,2.5,5,10,15,20 \mu \mathrm{m}$. It is ensured that the bubble mattress always consists of an integer number of bubble units $N$.

The following expression gives the effective slip length $b$ as function of the average pressure gradient $\delta p / \delta x$ over the middle $360 \mu \mathrm{m}$ of the bubble mattress, the fluid flux $\phi$, the channel height $H$, and the liquid viscosity $\mu$ * $^{*}$

$$
b=\left(-\phi-\frac{H^{2}}{12 \mu} \frac{\delta p}{\delta x}\right) /\left(\frac{\phi}{H}+\frac{H}{3 \mu} \frac{\delta p}{\delta x}\right) .
$$

Liquid flow over the bubble mattress is governed by the continuity equation and the Navier-Stokes equation for incompressible and steady flow,

$$
\begin{gathered}
\nabla \cdot \mathbf{u}=0, \\
\rho \mathbf{u} \cdot \nabla \mathbf{u}=-\nabla p+\mu \nabla^{2} \mathbf{u},
\end{gathered}
$$

where $\rho$ is the fluid density, $\mathbf{u}$ is the velocity vector, $p$ is the pressure and $\mu$ is the liquid viscosity. Solute transport is described by the convection-diffusion equation,

$$
\nabla \cdot(\mathbf{u} c)=\nabla \cdot(D \nabla c)
$$

where $c$ is the solute concentration, and $D$ is the diffusivity of the solute in the liquid.

For solving the governing equations, multiple boundary conditions (BCs) need

* Solving for two-dimensional Stokes flow with a no-slip BC at the upper wall and Navier's slip $\mathrm{BC}$ at the lower wall results in the following expression for the velocity profile:

$$
u(y)=\frac{1}{2 \mu} \frac{\Delta p}{\Delta x}\left(y^{2}-H y\right)+u_{b}\left(1-\frac{y}{H}\right),
$$

where $u_{b}$ is the effective slip velocity of the liquid at the partially slippery wall. Using Navier's law for $u_{b}$, Eq. (5.1), it can be derived that

$$
u_{b}=\left.b \frac{\partial u}{\partial y}\right|_{y=0}=-\frac{b H}{2 \mu} \frac{\Delta p}{\Delta x}\left(1+\frac{b}{H}\right)^{-1} .
$$

Furthermore, from $u(y)$ it is found that

$$
\phi=\langle u\rangle=\frac{1}{H} \int_{0}^{H} u(y) \mathrm{d} y=-\frac{H^{2}}{12 \mu} \frac{\Delta p}{\Delta x}+\frac{u_{b}}{2} .
$$

Substituting for $u_{b}$ and subsequent rewriting of this formula results in Eq. (5.4), being an expression for the effective slip length $b$. Note that $\Delta p / \Delta x \neq \delta p / \delta x$. 
(a) $c / c_{0}$

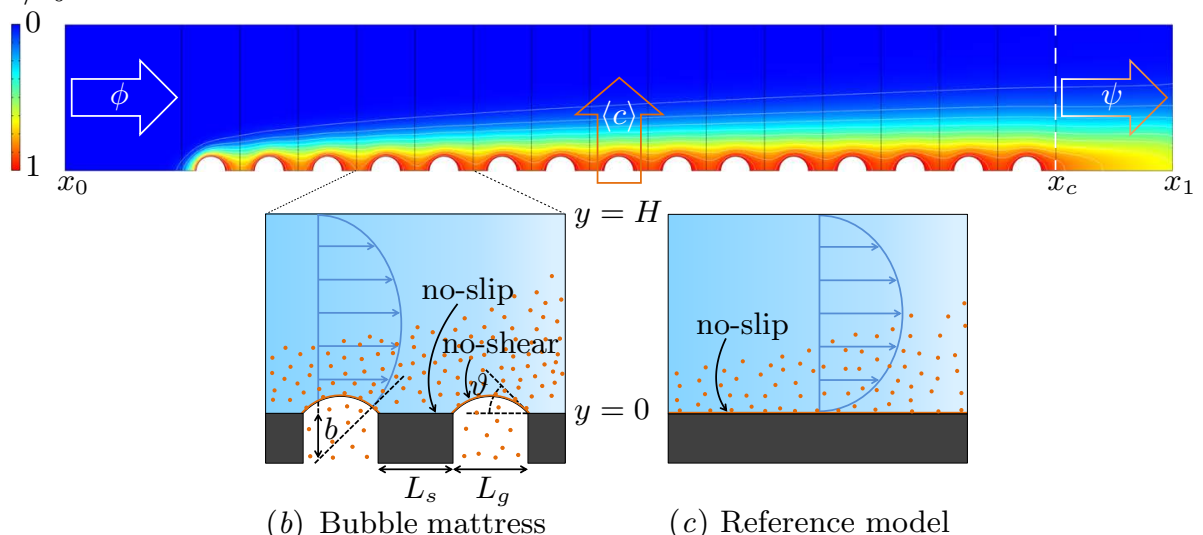

(b) Bubble mattress

(c) Reference model

Figure 5.1 - A solute concentration profile above the bubble mattress is provided in $(a)$, with the solute being supplied from the gas bubbles. Here the bubble size equals $L_{g}=20 \mu \mathrm{m}$. The surface porosity is $\varepsilon=1 / 2$. In $(b)$ a schematic depiction of the computational model is given, where the dots represent the dissolving solute. The solute concentration of $c=c_{0}$ at the bubble surface is fixed. A no-flux boundary condition is applied to the solid wall between the bubbles. In the simulations only the pressure-driven laminar flow is modelled. The reference model used for calculation of the mass transfer enhancement is shown in $(c)$, where $c=c_{0}$ at the non-slippery bottom wall.

to be specified. The boundary conditions for the in- and outlet are:

$$
\begin{aligned}
& p=p_{0} \text { at } x=x_{0}, \\
& p=p_{1} \text { at } x=x_{1},
\end{aligned}
$$

The viscous stress is set to zero at both inlet and outlet:

$$
\left[\mu\left(\nabla \mathbf{u}+(\nabla \mathbf{u})^{\mathrm{T}}\right)\right] \mathbf{n}=0 \text { at } x=x_{0} \text { and } x=x_{1} .
$$

A no-slip BC is applied to the solid wall between the bubbles, and to the upper wall:

$$
\mathbf{u}=\mathbf{0} \text { at } y=0 \text { (for } x \text { coinciding } L_{s} \text { ) and } y=H \text {. }
$$

The gas-liquid interface is a shear-free boundary (see Fig. 5.1(b)). The BCs are (where $\mathbf{I}$ is the $2 \times 2$ identity matrix):

$$
\mathbf{u} \cdot \mathbf{n}=0 \text { at bubble surface, }
$$




$$
\left[-P \mathbf{I}+\mu\left(\nabla \mathbf{u}+(\nabla \mathbf{u})^{\mathrm{T}}\right)\right] \mathbf{n}=0
$$

The solute concentration $c$ at the inlet equals zero:

$$
c=0 \text { at } x=x_{0} .
$$

A constant solute concentration $c_{0}$ is imposed on the bubble surface:

$$
c=c_{0} \text { at bubble surface. }
$$

It is assumed that the solute flux at the outlet is dominated by convective transport, which results in the following $\mathrm{BC}$,

$$
\mathbf{n} \cdot(-D \nabla c)=0 \text { at } x=x_{1},
$$

where $\mathbf{n}$ is the vector normal to the surface. Finally, there is no solute flux through the lower wall for $x$ coinciding $L_{s}$, nor through the upper wall:

$$
\mathbf{n} \cdot(-D \nabla c+\mathbf{u} c)=0 \text { at } y=0 \text { (for } x \text { coinciding } L_{s} \text { ) and } y=H \text {. }
$$

For solving the governing equations together with the boundary conditions, the finite-element methods software package COMSOL Multiphysics 4.1 was utilised. Flow fields and concentration profiles were computed sequentially in two steps. Meshing of the numerical models was performed automatically, where the element size was optimised for fluid dynamics with maximum and minimum element sizes of respectively $1.3 \mu \mathrm{m}$ and $0.15 \mu \mathrm{m}$. Near boundaries, smaller element sizes were used with maximum and minimum sizes of respectively $0.67 \mu \mathrm{m}$ and $0.020 \mu \mathrm{m}$. The number of mesh elements amounted to $1.5 \times 10^{5}$ for a model with $L_{g}=20 \mu \mathrm{m}$, and to $5.6 \times 10^{5}$ for a model with $L_{g}=1 \mu \mathrm{m}$. Generally, the number of elements was larger with decreasing $L_{g}$ and increasing $\varepsilon$. A parametric sweep was employed to investigate the amount of effective wall slip and mass transfer enhancement for $-90^{\circ}<\vartheta<90^{\circ}$ using angle increments of $8^{\circ}$.

\subsubsection{Reference models}

To evaluate the mass transfer characteristics for fluid flow over a bubble mattress, the mass transfer enhancement $E$ is calculated with respect to the reference model shown in Fig. 5.1(c). This model represents two-dimensional fluid flow between flat, non-slippery parallel plates, where a constant solute concentration $c_{0}$ is applied to the lower plate.

The mass transfer enhancement is calculated using the solute flux $\psi$ directly 
after the last bubble unit, i.e. at $x=x_{c}$ (see Fig. 5.1(a)),

$$
\psi=\frac{1}{H} \int_{0}^{H} u(y) c(y) \mathrm{d} y=\phi\langle c\rangle,
$$

which equals the product of fluid flux $\phi$ and flow-averaged or mixing-cup outlet solute concentration $\langle c\rangle . \phi$ and $\langle c\rangle$ are given by the following equations:

$$
\begin{gathered}
\phi=\frac{1}{H} \int_{0}^{H} u(y) \mathrm{d} y \\
\langle c\rangle=\int_{0}^{H} u(y) c(y) \mathrm{d} y / \int_{0}^{H} u(y) \mathrm{d} y .
\end{gathered}
$$

The solute flux enhancement $E^{\psi}$ is defined as the ratio of the solute flux of the bubble mattress $\psi$ to the solute flux of the reference model $\psi_{r}$ :

$$
E^{\psi}=\psi / \psi_{r}
$$

Analogous to the solute flux enhancement $E^{\psi}$, the fluid flux enhancement $E^{\phi}$ and the flow-averaged solute outlet concentration enhancement $E^{c}$ are defined:

$$
\begin{gathered}
E^{\phi}=\phi / \phi_{r}, \\
E^{c}=\langle c\rangle /\langle c\rangle_{r} .
\end{gathered}
$$

Evaluation of $E^{\phi}$ and $E^{c}$ is valuable to investigate the major source of the solute flux enhancement, since

$$
E^{\psi}=E^{\phi} E^{c}
$$

\subsection{Results and discussion}

\subsubsection{Momentum transport}

The extent of effective wall slip affects the ultimate liquid flow or momentum transport over the bubble mattress. In Fig. 5.2 the dimensionless effective slip length $2 b / L_{g}$ is plotted as a function of the bubble protrusion angle $\vartheta$ for three different surface porosities $\varepsilon$.

The slip length profiles reveal a qualitative difference between convex and concave gas bubbles. The slip length is always positive for $\vartheta<0$. For $\vartheta>0$ both positive and negative slip is observed. The profiles can be divided into two regions, 


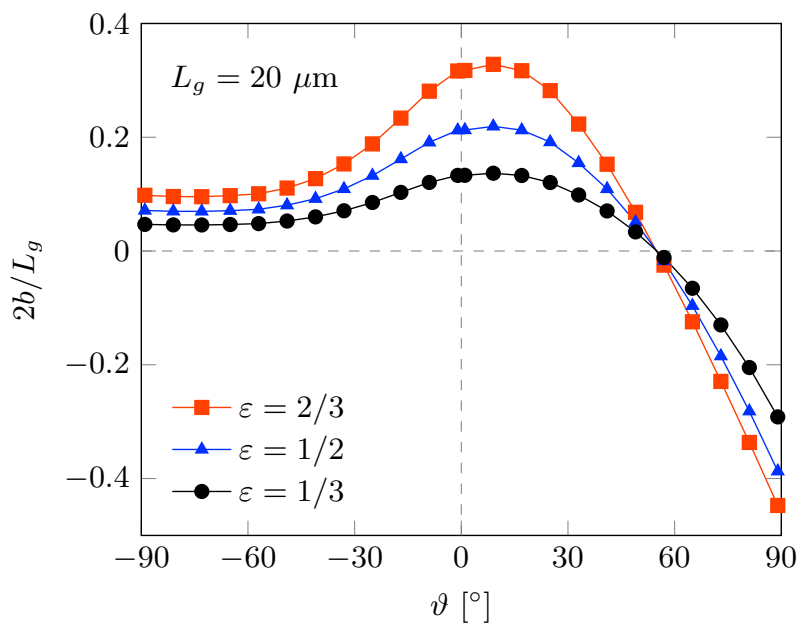

Figure 5.2 - Dimensionless effective slip length $2 b / L_{g}$ as a function of the protrusion angle $\vartheta$ for three different surface porosities $\varepsilon=L_{g} / L$.

separated by a critical protrusion angle $\vartheta_{c}=55^{\circ}$. For $\vartheta<\vartheta_{c}$ the slip length is positive, i.e. reduced hydrodynamic friction/drag; for $\vartheta>\vartheta_{c}$ slip lengths are negative, i.e. increased hydrodynamic friction/drag.

The course of the slip length profiles can be explained by means of the flow fields for three different protrusion angles that are provided in Fig. 5.3. For negative $\vartheta$, the liquid inside the bubble pockets becomes stagnant: see the flow profile for $\vartheta=-65^{\circ}$ in Fig. 5.3( $\left.a\right)$. As a result, the porous wall at $y=0$ approaches an alternating solid-liquid wall. Since internal liquid friction is smaller than friction between liquid and wall, $b$ approaches a plateau value when $-90^{\circ}<\vartheta<-60^{\circ}$, but remains positive.

Wall slip is maximum when $-20^{\circ}<\vartheta<30^{\circ}$ for all $\varepsilon$. For $\varepsilon=2 / 3$ the maximum slip length equals $16 \%$ of the bubble size $L_{g}$. The flow profile in Fig. 5.3(b) for $\vartheta=1^{\circ}$ illustrates why $b$ is maximum for moderately positive $\vartheta$. The slippery bubble surface results in a higher effective liquid velocity close to the lower wall, without significantly reducing the effective channel height. This minimises $\delta p / \delta x$ and maximises $\phi$ over the bubble mattress.

When the bubble protrusion becomes larger, $b$ gets smaller. The slip length $b$ is positive as long as $\vartheta<\vartheta_{c}$; for $\vartheta_{c}$ the resistance to fluid flow as a result of geometric flow obstruction (see e.g. Fig. 5.3(c) for $\vartheta=65^{\circ}$ ) is just counteracted by liquid slip over the bubbles. For $\vartheta>\vartheta_{c}$ the resistance caused by flow obstruction is dominant, and as a consequence $b$ turns negative. The critical protrusion angle of $55^{\circ}$ found in this study is slightly smaller than those found in other studies, which are in the range of $62^{\circ}$ to $69^{\circ}[18,27,34,36]$. However, these studies consider 


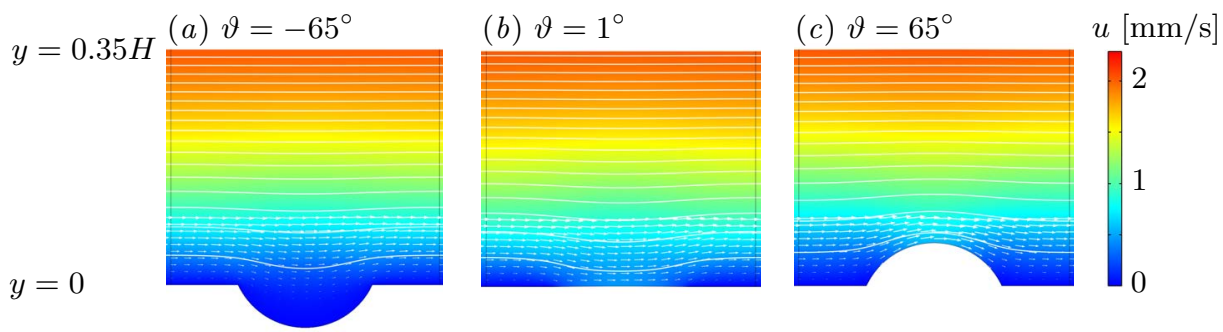

Figure 5.3 - Flow fields for the protrusion angles of $(a) \vartheta=-65^{\circ},(b) \vartheta=1^{\circ}$, and (c) $\vartheta=65^{\circ}$, where the surface porosity is $\varepsilon=1 / 2$. The velocity field is visualised with both stream lines and arrows. For highly negative $\vartheta$ the bubbles can be considered as a cavity filled with a stagnant liquid. For moderate angles $\left(-20^{\circ}<\vartheta<30^{\circ}\right)$ the fluid flow is significantly enhanced by the slippery bubbles. For large $\vartheta$ the protruding bubbles obstruct the flow, wherefore effective slip is negative for $\vartheta>55^{\circ}$.

periodic Couette flow, while our simulations concern non-periodic pressure-driven flow. It is worth mentioning that we also found a critical angle of $62^{\circ}$ in our periodic simulations for Couette flow. The different flow configurations may explain why a lower $\vartheta_{c}$ is obtained for pressure-driven flow.

The slip lengths in Fig. 5.2 are plotted for three different surface porosities, which show that effective slip becomes larger when increasing $\varepsilon$. A larger $\varepsilon$ means an increase in the relative area of the slippery gas-liquid interface, which in turn reduces the hydrodynamic dissipation. This ultimately results in larger effective slip lengths.

The dilute limit model of Davis and Lauga, Eq. (5.2), predicts that the dimensionless slip length depends linearly on the porosity [18]. For larger porosities however $(\varepsilon>0.35)$, this model underestimates the amount of effective slip, although the predicted dependency of slip on the protrusion angle remains correct qualitatively [43]. This is in agreement with our results, which also reveal that $\left(2 b / L_{g}\right) / \varepsilon$ gets larger for increasing $\varepsilon$. This non-linear increase in effective slip may be explained by considering that for high surface porosities the liquid between the bubbles starts to become stagnant. As a result, the liquid flows over a hybrid surface that is characterised by an alternating pattern of gas-liquid instead of gas-solid. Because internal liquid friction is smaller than friction between liquid and solid, the flow resistance decreases non-linearly. This is supported by the observation that for $\vartheta<-60^{\circ}$ the relation between $b$ and $\varepsilon$ is linear, because of the liquid inside the bubble pockets being stagnant for all $\varepsilon$.

Finally, our numerical results show that for a given porosity the dimensionless slip length is independent of the applied pressure gradient $\Delta p / \Delta x$ (in the laminar regime), the gas bubble size $L_{g}$, the bubble unit length $L$, and the number of bubble units $N$. 


\subsubsection{Interfacial mass transport}

Mass transfer enhancement. To investigate the effect of a slippery bubble mattress on interfacial mass transport, the ratio of the solute flux at the end of the bubble mattress to that obtained for a flat, fully saturated no-slip wall has been computed. This ratio is referred to as the solute flux enhancement $E^{\psi}$. Note that for a given pressure gradient both momentum and mass transport are constant in the reference model. The pressure gradient for the bubble mattress has been chosen such, that the solute concentration at the upper wall is negligible. This ensures that the ultimate mass transfer is not affected by accumulation of the solute at the upper wall. Since $E^{\psi}=E^{\phi} E^{c}$, the fluid flux enhancement $E^{\phi}$ and flow-averaged solute concentration enhancement $E^{c}$ have been evaluated to identify the major source contributing to the solute flux enhancement. An example of a concentration profile is shown in Fig. 5.1(a). The resulting enhancement profiles for a bubble mattress with $L_{g}=20 \mu \mathrm{m}$ are provided in Fig. 5.4.

As the profiles in Fig. 5.4(a) reveal, the dependency of the solute flux enhancement $E^{\psi}$ on the protrusion angle $\vartheta$ is qualitatively similar for all surface porosities $\varepsilon$. Three regions can be identified, where the boundaries of these domains are based on the fluid flux enhancement $E^{\phi}$ profiles in Fig. 5.4(b). In domain I, where $-90^{\circ}<\vartheta<-30^{\circ}, E^{\psi}$ is smaller than $1\left(E^{\phi} \approx 1\right)$. The solute flux enhancement increases approximately linearly with $\vartheta$. From $\vartheta=-30^{\circ}$ up to $\vartheta=55^{\circ}$, which is domain II, $E^{\psi}$ first increases more than linearly, and then starts to decline $\left(E^{\phi}>1\right)$. For $\varepsilon=2 / 3, E^{\psi}$ is optimal in this region. In domain III, where $55^{\circ}<\vartheta<90^{\circ}$, the increase in solute flux enhancement is either constant or gradually declining $\left(E^{\phi}<1\right)$. As Fig. 5.4(c) shows, the solute concentration enhancement $E^{c}$ increases approximately linearly for all $\vartheta$.

In domain I, the solute concentration above the bubble mattress is considerably lower than that above a fully saturated no-slip wall $\left(E^{c}<1\right)$. This is caused by the stagnancy of the liquid inside the bubble pockets (see Fig. 5.3 $(a)$ ), which also explains why $E^{\phi}$ is constant in this regime. As a consequence, solute transport from the bubble surface towards the main channel is dominated by diffusion in the vertical direction. Increasing $\vartheta$ reduces this distance, which clarifies why an increase in $\vartheta$ directly results in a larger $E^{c}$ and $E^{\psi}$.

For moderate bubble protrusion angles, i.e. for $\vartheta$ in domain II, effective wall slip and accordingly $E^{\phi}$ are maximal. As a result, interfacial solute transport occurs by both diffusion and convection. The large effective wall slip not only enhances mass uptake by an increase of the fluid flow in the $x$-direction (large $E^{\phi}$ ), but also by the interface-induced $y$-directional liquid velocity (see Fig. 5.3(b)). Besides the growing protrusion depth of the bubbles (large $E^{c}$ ), the enhanced solute transport in both $x$ - and $y$-direction results in a considerable increase of the solute flux enhancement $E^{\psi}$. The fact that only a porosity of $\varepsilon>0.5$ is required for $E^{\psi}>1$ for positive $\vartheta$ reflects the significance of convective interfacial 

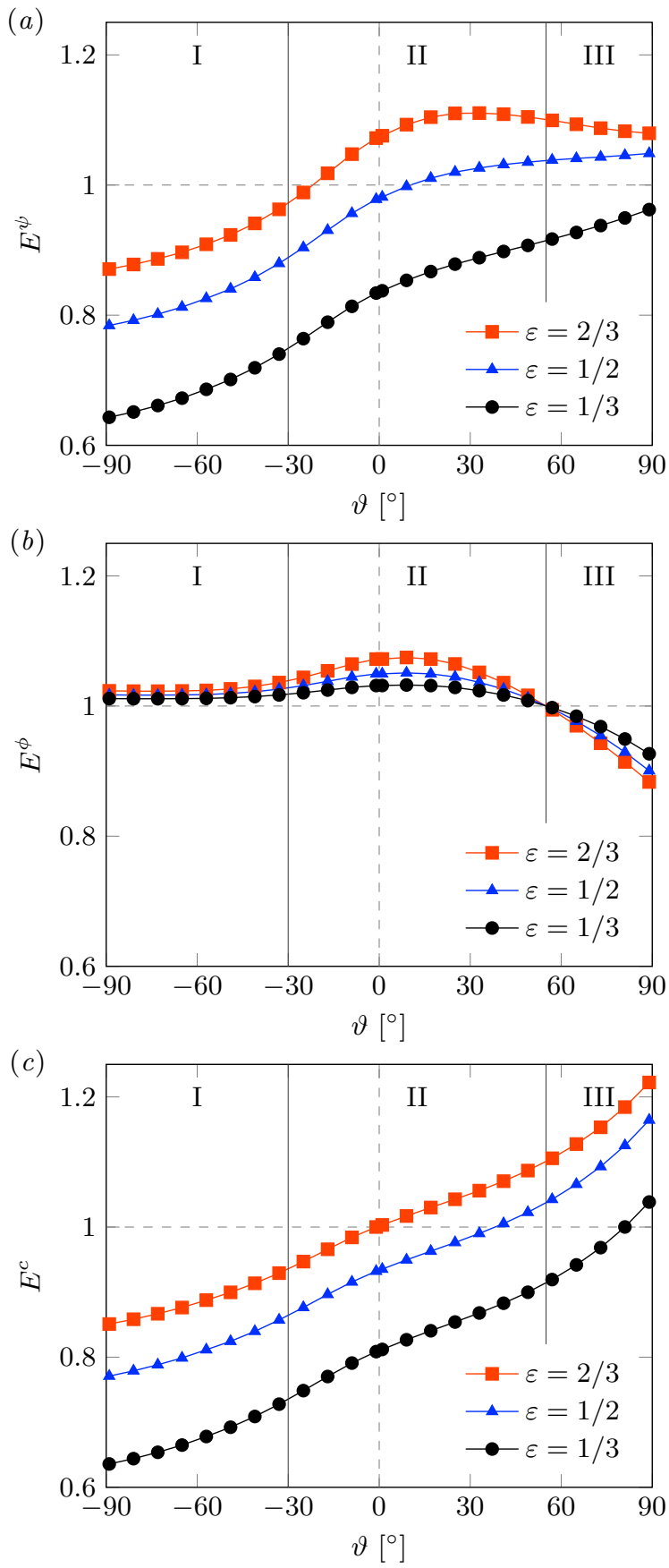

Figure 5.4 - The effect of protrusion angle $\vartheta$ and surface porosity $\varepsilon$ on the $(a)$ solute flux enhancement $E^{\psi},(b)$ fluid flux enhancement $E^{\phi}$, and $(c)$ flow-averaged solute concentration enhancement $E^{c}$. Here $L_{g}=20 \mu \mathrm{m}$. For a given $\varepsilon$ and $\vartheta, E^{\psi}=E^{\phi} E^{c}$. 
solute transport. Contrary to $E^{c}, E^{\phi}$ starts to decline for $\vartheta>10^{\circ}$. This is due to the increasing obstruction of the flow by the protruding bubbles. Therefore the $E^{\psi}$-profile displays a maximum enhancement of $11 \%$ at $\vartheta=25^{\circ}$ for $\varepsilon=2 / 3$. Note that the maximum enhancement is specific for the geometry and conditions defined in the numerical model.

Effective wall slip is negative for protrusion angles in domain III. Therefore $E^{\phi}<1$. However, the solute concentration above the bubble mattress continues to grow with increasing $\vartheta$ as a result of both the increasing bubble area and the bubble protrusion depth. The latter situation entails that the solute enters the liquid in faster flowing lamina. Moreover, the wavy fluid flow just above the bubble mattress enhances convective solute transport in the $y$-direction (see Fig. 5.3(c)). Hence the solute flux enhancement $E^{\psi}$ remains growing for roughly $\varepsilon<0.5$, or approaches a plateau value larger than 1 for $\varepsilon>0.5$.

Fig. 5.4(a) shows that $E^{\psi}$ depends non-linearly on the surface porosity $\varepsilon$. This reflects the non-linear correlation between $\varepsilon$ and both $E^{\phi}$ and $E^{c}$. The dependency of $E^{\phi}$ on the porosity is similar to the previously discussed correlation between the effective slip length $b$ and $\varepsilon$. The non-linear dependency of $E^{c}$ on $\varepsilon$ is the result of two effects. First, the average solute concentration above the bubble mattress is low due to the small solute exchange area for the bubble mattress compared to that in the reference model. Second, for low $\varepsilon$ the fluid velocity just above the bubble mattress is small, since $b \sim \varepsilon$. Both effects cause the flow-averaged solute concentration $\langle c\rangle$ to grow more than proportional upon an increase of $\varepsilon$ for small porosities (Eq. (5.20)). For large $\varepsilon$ however, the solute concentration above the bubble mattress approaches full saturation as a result of the non-zero liquid velocity above the bubble mattress. This minimises concentration variations along the bubble mattress by convective solute transport. Therefore the limit of full saturation can be reached when $\varepsilon<1$.

To summarise, the solute flux enhancement $E^{\psi}$ can be divided into three domains. For $-90^{\circ}<\vartheta<-30^{\circ}$ interfacial solute transport is diffusion limited, wherefore $E^{\psi}<1$ for $\varepsilon=2 / 3$. For $-30^{\circ}<\vartheta<55^{\circ}$ solute transport is convection enhanced as a result of significant effective wall slip, and hence $E^{\psi}>1$ for $\varepsilon=2 / 3$. For $55^{\circ}<\vartheta<90^{\circ}$, where the bubble mattress shows increased hydrodynamic friction drag, mass transport is geometry enhanced $\left(E^{\psi}>1\right.$ for $\left.\varepsilon=2 / 3\right)$ due to the deep protrusion of the bubbles in the main channel flow.

The influence of bubble size. To investigate the influence of bubble size on the solute flux enhancement, simulations have been performed for bubbles in the size range of $1 \leq L_{g} \leq 20 \mu \mathrm{m}$ for three different surface porosities. The resulting profiles for $\varepsilon=2 / 3$ (which is a typical porosity for microporous membranes) [50] constitute the surface plot shown in Fig. 5.5. This graph demonstrates that a decreasing bubble size results in a convergence of $E^{\psi}$ towards 1 . To understand this behaviour, we defined a local Péclet number at the bottom wall to consider 


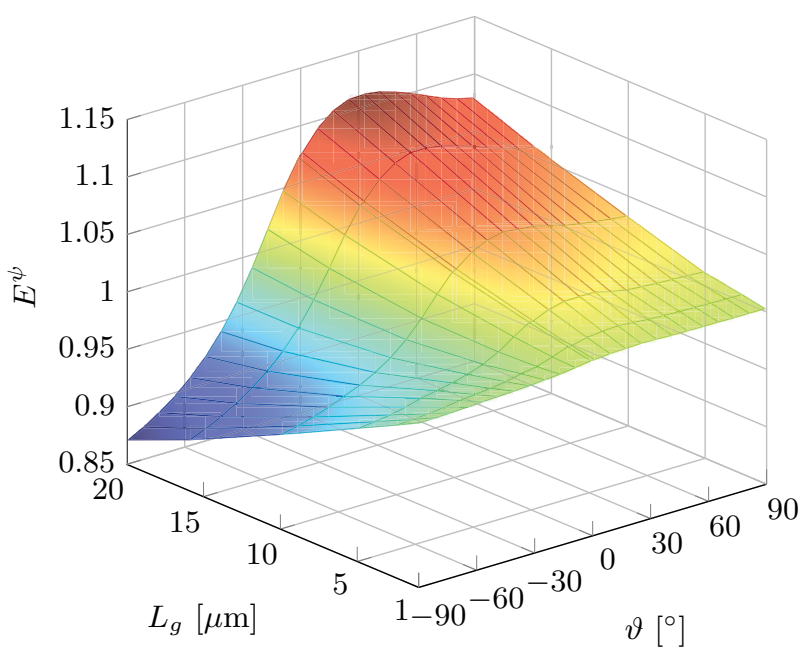

Figure 5.5 - Solute flux enhancement $E^{\psi}$ as a function of protrusion angle $\vartheta$ and bubble size $L_{g}$ for a surface porosity of $\varepsilon=2 / 3$.

the ratio of convective to diffusive transport between the solute-saturated bubbles. This local Péclet number is defined as:

$$
P e=\frac{L_{s} u_{b}}{D}
$$

When $P e<1$, solute transport is diffusion dominated. It is expected that this results in $E^{\psi}<1$. Consequently, solute transport is convection dominated for $P e>1$, which would then result in $E^{\psi}>1$. In order to estimate the bubble size $L_{g}$ where $P e=1$, Eq. (5.25) is rewritten as follows. First, the effective liquid velocity $u_{b}$ at the slippery wall is given by:

$$
u_{b}=-\frac{b H}{2 \mu} \frac{\Delta p}{\Delta x}\left(1+\frac{b}{H}\right)^{-1} .
$$

From the analytical model for effective wall slip of Davis and Lauga [18], see Eq. (5.2), it is derived that for flat bubbles, which is used as an approximation here,

$$
\frac{2 b}{\varepsilon L_{g}} \approx \frac{\pi}{8} \text { for } \vartheta=0^{\circ} .
$$

Substituting Eq. (5.26) and (5.27) in Eq. (5.25) results in the following expression for $P e$ :

$$
P e=-\frac{\pi}{32} \frac{L_{g}^{2}(1-\varepsilon) H}{\mu D} \frac{\Delta p}{\Delta x}\left(1+\frac{b}{H}\right)^{-1} .
$$


Since for this model $b \ll H$, this equation can be simplified to:

$$
P e \approx-\frac{\pi}{32} \frac{L_{g}^{2}(1-\varepsilon) H}{\mu D} \frac{\Delta p}{\Delta x} .
$$

Evaluation of this expression for $P e=1$ with $\Delta p / \Delta x=-1800 \mathrm{~Pa} / \mathrm{m}, H=$ $100 \mu \mathrm{m}, \varepsilon=2 / 3, \mu=10^{-3} \mathrm{~Pa}$ s and $D=10^{-9} \mathrm{~m}^{2} / \mathrm{s}$ gives a critical bubble size of $L_{c}=13 \mu \mathrm{m}$. In Fig. 5.6(a) the solute flux enhancement is plotted as function of $L_{g}$ for various protrusion angles. It is observed that, irrespective of protrusion angle, the solute flux enhancement approaches unity for $L_{g}<10 \mu \mathrm{m}$. This value of $L_{g}$ corresponds with the estimated critical bubble size of $L_{c}=13 \mu \mathrm{m}$.

Only large bubbles (i.e. $L_{g}>L_{c}$ ) can lead to a significant enhancement of solute transport. For very small bubbles however, the effective slip length approaches zero and consequently the bubble mattress behaves as a no-slip wall. Furthermore, since the distance between the bubbles becomes very small, diffusional transport is fast enough to result in a fully solute-saturated wall, especially because the rate of diffusional transport remains constant. These two effects imply that solute transport over a bubble mattress characterised by very small bubbles (i.e. $\left.L_{g}<L_{c}\right)$ must approach that over a fully saturated no-slip wall.

We can now extend our analysis to understand the observation of Kreulen et al. [49] that in hollow fibre membranes solute transport is independent of the surface porosity. For partially slippery surfaces like their membranes, which are characterised by pore sizes of $0.1 \mu \mathrm{m}$, the slip velocity at the wall approaches zero. This results in estimated local Péclet numbers as small as $P e \sim 10^{-2}$. Because for $P e<1$ diffusion is the dominant transport mechanism, the wall is solute saturated, and no influence of the membrane porosity on mass transfer is found.

For bubble sizes larger than approximately $10 \mu \mathrm{m}$, solute transport is convection enhanced. Although in that case $P e>1$, due to effective wall slip the solute concentration above the solid wall between the bubbles does approach saturation. The fluid flow parallel to the wall smoothes out concentration variations along the bubble mattress (see Fig. 5.1(a)). This process is also observed by Juhasz and Deen $[47,48]$. It provides an explanation why the solute flux enhancement $E^{\psi}$ depends non-linearly on the surface porosity $\varepsilon$, as shown in Fig. 5.6(b). Moreover, this figure illustrates that the critical bubble size decreases for smaller $\varepsilon$, which is in agreement with Eq. (5.29). Due to the large distance between the bubbles for low porosities, it requires smaller bubble sizes to let diffusion dominate over convective transport.

In summary, the criticial bubble size $L_{c}$ at which locally $x$-directional convective transport equals diffusive transport can be estimated by the following equation:

$$
L_{c} \approx\left(-\frac{32}{\pi} \frac{\mu D}{(1-\varepsilon) H} \frac{\Delta x}{\Delta p}\right)^{1 / 2} .
$$



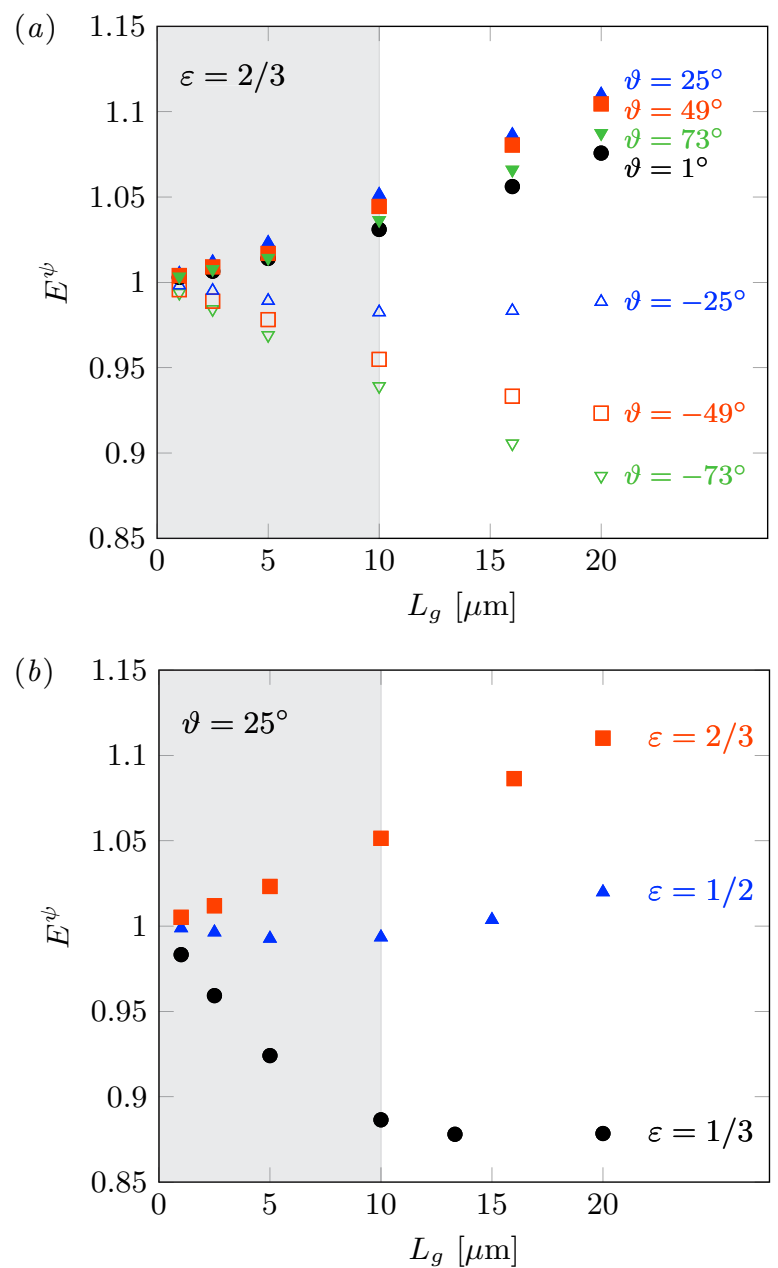

Figure 5.6 - Solute flux enhancement $E^{\psi}$ as a function of the bubble size $L_{g}$ for a varying ( $a$ ) protrusion angle $\vartheta$ and $(b)$ surface porosity $\varepsilon$.

It follows that when $L_{g}<L_{c}$, where $P e<1$, local solute transport is diffusion dominated. Consequently, when $L_{g}>L_{c}$ and hence $P e>1$, solute transport near the partially slippery bubble mattress is advection assisted, thereby enhancing solute transport.

To address the dependency of the local Péclet number on the flow conditions in the channel, Eq. (5.4) is rewritten to obtain an expression for $\Delta p / \Delta x$ :

$$
\frac{\Delta p}{\Delta x}=-\frac{12 \mu\langle u\rangle}{H^{2}} \frac{(H+b)}{(H+4 b)} .
$$


This equation is subsequently used to rewrite Eq. (5.28) as

$$
P e=\frac{3 \pi}{8}(1-\varepsilon) \operatorname{ReSc}\left(\frac{L_{g}}{H}\right)^{2}\left(1+\frac{\pi}{4} \frac{\varepsilon L_{g}}{H}\right)^{-1}
$$

where $R e=(\rho\langle u\rangle H) / \mu$ is the Reynolds number, and $S c=\mu /(\rho D)$ is the Schmidt number. From this expression it follows that an increase in Reynolds number results in a higher local Péclet number, which is in agreement with previously published results $[47,48]$. It also shows that the ratio of bubble size to channel height, i.e. the bubble confinement $L_{g} / H$, is an important factor. A larger bubble confinement increases the effective liquid velocity $u_{b}$ at the partially slippery wall, and consequently also the local Péclet number. Our simulation results for various Reynolds numbers (varying $\Delta p / \Delta x$ ) and channel heights confirm the dependency of the local Péclet number on the Reynolds number and the bubble confinement qualitatively.

\subsection{Conclusions}

We have investigated the influence of the interface geometry of a partially slippery bubble mattress on interfacial mass transport. Simulations for two-dimensional steady pressure-driven flow over a bubble mattress show that the dependency of effective wall slip on the bubble protrusion angle $\vartheta$ and surface porosity $\varepsilon$ is consistent with previously published results for Couette flow, albeit a stronger dependency of effective slip on the protrusion angle is found for our pressure-driven flow configuration.

To examine the effect of the bubble mattress on interfacial mass transfer, the obtained solute flux is compared with that for a solute-saturated no-slip wall. The resulting solute flux enhancement profiles can be divided into three domains. For $-90^{\circ}<\vartheta<-30^{\circ}$ solute transport is diffusion limited. Solute transport is slip enhanced for $-30^{\circ}<\vartheta<55^{\circ}$. For $55^{\circ}<\vartheta<90^{\circ}$, where the effective slip length is negative, solute transport is geometry enhanced as a result of the deep bubble protrusion in the fluid flow. Maximum flux enhancements are obtained for moderately positive protrusion angles. The maximum enhancement for a mattress with bubbles having a width of $L_{g}=20 \mu \mathrm{m}$ amounts to $11 \%$ for $\varepsilon=2 / 3$. A stronger enhancement can be obtained for larger bubbles, higher surface porosities and increased fluid flows.

Finally, we provide a theoretical analysis, based on the local Péclet number, explaining why the solute flux enhancement approaches unity for a decreasing bubble size. Effective wall slip vanishes for very small bubbles, wherefore diffusive transport becomes faster than convective transport at the scale of the microscopic bubble. Consequently, in that case, the porous and slippery bubble mattress can 
be considered as a fully saturated no-slip wall. The critical bubble size depends not only on the interface geometry, but also on the bubble confinement and on the flow conditions over the bubble mattress.

We believe that these partially slippery surfaces, since they can be tuned and optimised to enhance momentum and mass transport simultaneously [33], have considerable potential applicability in both micro- and macroscale gas-liquid processes.

\section{References}

[1] H. A. Stone, A. D. Stroock, and A. Ajdari, Engineering flows in small devices, Annu. Rev. Fluid Mech. 36, 381 (2004).

[2] L. Bocquet and J.-L. Barrat, Flow boundary conditions from nano- to microscales, Soft Matter 3, 685 (2007).

[3] L. Bocquet and E. Lauga, A smooth future?, Nat. Mater. 10, 334 (2011).

[4] A. Ajdari and L. Bocquet, Giant amplification of interfacially driven transport by hydrodynamic slip: diffusio-osmosis and beyond, Phys. Rev. Lett. 96, 186102 (2006).

[5] L. Joly, C. Ybert, E. Trizac, and L. Bocquet, Hydrodynamics within the electric double layer on slipping surfaces, Phys. Rev. Lett. 93, 257805 (2004).

[6] T. M. Squires, Electrokinetic flows over inhomogeneously slipping surfaces, Phys. Fluids 20, 092105 (2008).

[7] H. Zhao, Electro-osmotic flow over a charged superhydrophobic surface, Phys. Rev. E 81, 066314 (2010).

[8] L. Biswal, S. K. Som, and S. Chakraborty, Thin film evaporation in microchannels with interfacial slip, Microfluid. Nanofluid. 10, 155 (2011).

[9] G. S. Beavers and D. D. Joseph, Boundary conditions at a naturally permeable wall, J. Fluid Mech. 30, 197 (1967).

[10] S. Chellam, M. R. Wiesner, and C. Dawson, Slip at a uniformly porous boundary: effect on fluid flow and mass transfer, J. Eng. Math. 26, 481 (1992).

[11] G. Ramon, Y. Agnon, and C. Dosoretz, Heat transfer in vacuum membrane distillation: effect of velocity slip, J. Membr. Sci. 331, 117 (2009).

[12] E. Karatay and R. G. H. Lammertink, Oxygenation by a superhydrophobic slip G/L contactor, Lab Chip 12, 2922 (2012).

[13] J. M. Jani, M. Wessling, and R. G. H. Lammertink, A microgrooved membrane based gas-liquid contactor, Microfluid. Nanofluid. 13, 499 (2012).

[14] R. Singh and R. L. Laurence, Influence of slip velocity at a membrane surface on ultrafiltration performance - I. Channel flow system, Int. J. Heat Mass Transfer 22, 721 (1979). 
[15] H. M. Yeh and T. W. Cheng, Analysis of the slip effect on the permeate flux in membrane ultrafiltration, J. Membr. Sci. 154, 41 (1999).

[16] M. F. Gruber, C. J. Johnson, C. Y. Tang, M. H. Jensen, L. Yde, and C. Hélix-Nielsen, Computational fluid dynamics simulations of flow and concentration polarization in forward osmosis membrane systems, J. Membr. Sci. 379, 488 (2011).

[17] E. Lauga, M. P. Brenner, and H. A. Stone, in Springer handbook of experimental fluid mechanics, edited by C. Tropea, A. L. Yarin, and J. F. Foss (Springer Berlin Heidelberg, 2007), pp. 1219-1240.

[18] A. M. J. Davis and E. Lauga, Geometric transition in friction for flow over a bubble mattress, Phys. Fluids 21, 011701 (2009).

[19] C. L. M. H. Navier, Mémoire sur les lois du mouvement des fluids, Mem. Acad. Sci. Inst. Fr. 6, 389 (1823).

[20] J. P. Rothstein, Slip on superhydrophobic surfaces, Annu. Rev. Fluid Mech. 42, 89 (2010).

[21] O. I. Vinogradova and A. V. Belyaev, Wetting, roughness and flow boundary conditions, J. Phys.: Condens. Matter 23, 184104 (2011).

[22] K. Watanabe, Y. Udagawa, and H. Udagawa, Drag reduction of newtonian fluid in a circular pipe with a highly water-repellent wall, J. Fluid Mech. 381, 225 (1999).

[23] C. Cottin-Bizonne, J.-L. Barrat, L. Bocquet, and E. Charlaix, Low-friction flows of liquid at nanopatterned interfaces, Nat. Mater. 2, 237 (2003).

[24] J. Ou, B. Perot, and J. P. Rothstein, Laminar drag reduction in microchannels using ultrahydrophobic surfaces, Phys. Fluids 16, 4635 (2004).

[25] J. Ou and J. P. Rothstein, Direct velocity measurements of the flow past drag-reducing ultrahydrophobic surfaces, Phys. Fluids 17, 103606 (2005).

[26] D. Maynes, K. Jeffs, B. Woolford, and B. W. Webb, Laminar flow in a microchannel with hydrophobic surface patterned microribs oriented parallel to the flow direction, Phys. Fluids 19, 093603 (2007).

[27] A. Steinberger, C. Cottin-Bizonne, P. Kleimann, and E. Charlaix, High friction on a bubble mattress, Nat. Mater. 6, 665 (2007).

[28] P. Gao and J. J. Feng, Enhanced slip on a patterned substrate due to depinning of contact line, Phys. Fluids 21, 102102 (2009).

[29] P. Tsai, A. M. Peters, C. Pirat, M. Wessling, R. G. H. Lammertink, and D. Lohse, Quantifying effective slip length over micropatterned hydrophobic surfaces, Phys. Fluids 21, 112002 (2009).

[30] C. J. Teo and B. C. Khoo, Flow past superhydrophobic surfaces containing longitudinal grooves: effects of interface curvature, Microfluid. Nanofluid. 9, 499 (2010).

[31] C.-H. Choi and C.-J. Kim, Large slip of aqueous liquid flow over a nanoengineered superhydrophobic surface, Phys. Rev. Lett. 96, 066001 (2006). 
[32] C. Lee, C.-H. Choi, and C.-J. C. Kim, Structured surfaces for a giant liquid slip, Phys. Rev. Lett. 101, 064501 (2008).

[33] E. Karatay, A. S. Haase, C. W. Visser, C. Sun, D. Lohse, P. A. Tsai, and R. G. H. Lammertink, Control of slippage with tunable bubble mattresses, P. Natl Acad. Sci. USA 110, 8422 (2013).

[34] J. Hyväluoma and J. Harting, Slip flow over structured surfaces with entrapped microbubbles, Phys. Rev. Lett. 100, 246001 (2008).

[35] D. Legendre and C. Colin, Enhancement of wall friction by fixed cap bubbles, Phys. Fluids 20, 051704 (2008).

[36] J. Hyväluoma, C. Kunert, and J. Harting, Simulations of slip flow on nanobubble-laden surfaces, J. Phys.: Condens. Matter 23, 184106 (2011).

[37] J. R. Philip, Flows satisfying mixed no-slip and no-shear conditions, Z. Angew. Math. Phys. 23, 353 (1972).

[38] J. R. Philip, Integral properties of flows satisfying mixed no-slip and no-shear conditions, Z. Angew. Math. Phys. 23, 960 (1972).

[39] E. Lauga and H. A. Stone, Effective slip in pressure-driven stokes flow, J. Fluid Mech. 489, 55 (2003).

[40] C. Ybert, C. Barentin, C. Cottin-Bizonne, P. Joseph, and L. Bocquet, Achieving large slip with superhydrophobic surfaces: scaling laws for generic geometries, Phys. Fluids 19, 123601 (2007).

[41] C. J. Teo and B. C. Khoo, Analysis of stokes flow in microchannels with superhydrophobic surfaces containing a periodic array of micro-grooves, Microfluid. Nanofluid. 7, 353 (2009).

[42] A. V. Belyaev and O. I. Vinogradova, Effective slip in pressure-driven flow past super-hydrophobic stripes, J. Fluid Mech. 652, 489 (2010).

[43] C.-O. Ng and C. Y. Wang, Effective slip for stokes flow over a surface patterned with two- or three-dimensional protrusions, Fluid Dyn. Res. 43, 065504 (2011).

[44] S. Schmieschek, A. V. Belyaev, J. Harting, and O. I. Vinogradova, Tensorial slip of superhydrophobic channels, Phys. Rev. E 85, 016324 (2012).

[45] K. H. Keller and T. R. Stein, A two-dimensional analysis of porous membrane transport, Math. Biosci. 1, 421 (1967).

[46] W. A. Wakeham and E. A. Mason, Diffusion through multiperforate laminae, Ind. Eng. Chem. Fundam. 18, 301 (1979).

[47] N. M. Juhasz and W. M. Deen, Effect of local Peclet number on mass transfer to a heterogeneous surface, Ind. Eng. Chem. Res. 30, 556 (1991).

[48] N. M. Juhasz and W. M. Deen, Mass transfer in a tube with wall flux confined to evenly spaced discrete areas, Chem. Eng. Sci. 48, 1745 (1993).

[49] H. Kreulen, C. A. Smolders, G. F. Versteeg, and W. P. M. van Swaaij, Microporous hollow fibre membrane modules as gas-liquid contactors. part 1. physical mass transfer processes: A specific application: Mass transfer in 
highly viscous liquids, J. Membr. Sci. 78, 197 (1993).

[50] R. W. Baker, in Membrane technology and applications (John Wiley \& Sons, Chichester, 2004), Chap. 2, pp. 15-87.

[51] V. Y. Dindore, D. W. F. Brilman, and G. F. Versteeg, Hollow fiber membrane contactor as a gas-liquid model contactor, Chem. Eng. Sci. 60, 467 (2005). 



\title{
CHAPTER 6
}

\section{The Graetz-Nusselt problem extended to continuum flows with finite slip}

\begin{abstract}
Graetz and Nusselt studied heat transfer between a developed laminar fluid flow and a tube at constant wall temperature. Here, we extend the Graetz-Nusselt problem to dense fluid flows with partial wall slip. Its limits correspond to the classical problems for no-slip and no-shear flow. The amount of heat transfer is expressed by the local Nusselt number $N u_{x}$, which is defined as the ratio of convective to conductive radial heat transfer. In the thermally developing regime, $N u_{x}$ scales with the ratio of position $\tilde{x}=x / L$ to Graetz number $G z$, i.e. $N u_{x} \propto(\tilde{x} / G z)^{-\beta}$. Here, $L$ is the length of the heated or cooled tube section. The Graetz number $G z$ corresponds to the ratio of axial advective to radial diffusive heat transport. In the case of no-slip, the scaling exponent $\beta$ equals $1 / 3$. For no-shear flow, $\beta=1 / 2$. The results show that for partial slip, where the ratio of slip length $b$ to tube radius $R$ ranges from zero to infinity, $\beta$ transitions from $1 / 3$ to $1 / 2$ when $10^{-4}<b / R<10^{0}$. For partial slip, $\beta$ is a function of both position and slip length. The developed Nusselt number $N u_{\infty}$ for $\tilde{x} / G z>0.1$ transitions from 3.66 to 5.78 , the classical limits, when $10^{-2}<b / R<10^{2}$. A mathematical and physical explanation is provided for the distinct transition points for $\beta$ and $N u_{\infty}$.
\end{abstract}

This chapter has been published as A.S Haase, S.J. Chapman, P.A. Tsai, D. Lohse and R.G.H. Lammertink, The Graetz-Nusselt problem extended to continuum flows with finite slip, J. Fluid Mech. 764, R3 (2015). 


\subsection{Introduction}

The classical Graetz-Nusselt problem concerns a fluid of uniform temperature $T_{0}$ flowing in an insulated cylindrical tube. The flow is laminar and hydrodynamically fully developed, and has constant physical properties (see Fig. 6.1). At $x=0$ the fluid enters a tube section with a constant wall temperature $T_{1}$. Neglecting viscous dissipation and axial heat conduction, Graetz and Nusselt [1-3] independently found a mathematical solution to this problem, describing the temperature profile for $x>0$. The problem was solved for both a uniform [1] and a parabolic [2, 3] fluid velocity profile. From the temperature distribution the local heat flux at the wall can be obtained, which is commonly expressed by the local Nusselt number $N u_{x}$, a dimensionless heat transfer coefficient $[4,5]$. The Nusselt number

$$
N u_{x}=\frac{h_{x} D}{k}
$$

where $h_{x}$ is the local heat transfer coefficient, $D=2 R$ is the diameter of the tube and $k$ is the thermal conductivity, can be interpreted as the ratio of convective to conductive radial heat transfer $[6,7]$. The Nusselt number $N u_{x}$ is a function of the dimensionless downstream position $\tilde{x}=x / L, L$ being the length of the non-insulated section of the tube, and the Graetz number $G z$ only. The Graetz number is defined as $[6,7]$

$$
G z=\frac{u_{a v} D^{2}}{\alpha L}=\operatorname{RePr} \frac{D}{L}
$$

where $u_{a v}$ is the average fluid velocity and $\alpha$ is the thermal diffusivity. It is the product of the Reynolds number $\operatorname{Re}=u_{a v} D / \nu$, the Prandtl number $\operatorname{Pr}=\nu / \alpha$ and the ratio $D / L$, and can be interpreted as the ratio of axial advective to radial diffusive heat transport.

The temperature profiles found by Graetz and Nusselt involve infinite series in terms of eigenvalues and eigenfunctions. When $\tilde{x} / G z>0.1$, the first terms of the series solutions dominate. This results in a developed Nusselt number $N u_{\infty}=3.66$ for parabolic flow and $N u_{\infty}=5.78$ for plug flow [7]. The flow is said to be thermally fully developed. For $\tilde{x} / G z<0.01$, when the flow is thermally developing, and in particular when $x \rightarrow 0$, a very large number of terms are required to obtain the Nusselt number with sufficient accuracy. Lévêque [8] circumvented this problem by assuming that in the entrance region the thermal boundary layer is thin compared with the viscous boundary layer $[4,5,7]$. In that case curvature effects can be neglected. Furthermore, it can be assumed that the bulk extends to infinity, and that the velocity profile is linear. Lévêque showed that

$$
N u_{x} \propto\left(\frac{\tilde{x}}{G z}\right)^{-\beta},
$$



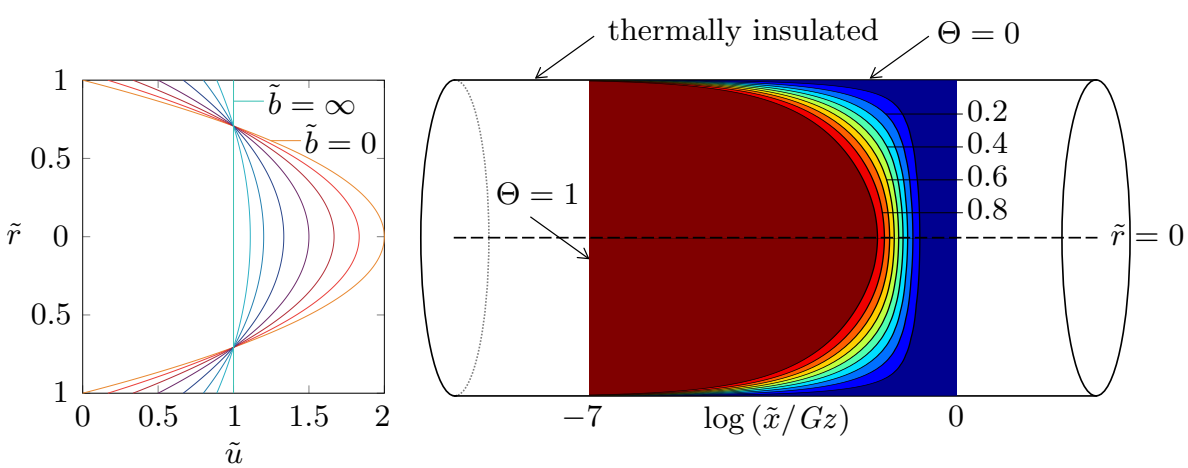

Figure 6.1 - The Graetz-Nusselt problem concerns transport of heat between a hydrodynamically fully developed fluid flow with $\Theta=\left(T_{1}-T\right) /\left(T_{1}-T_{0}\right)=1$ and a tube with a constant wall temperature $\Theta=0$ for $\tilde{x}>0$. The velocity profile of the inflowing fluid is a function of the tube radius $\tilde{r}$ and the slip length $\tilde{b}$, where $0 \leq \tilde{b} \leq \infty$. The profile is parabolic for $\tilde{b}=0$ and uniform for $\tilde{b}=\infty$. The contour map gives an example of a temperature profile.

with $\beta=1 / 3$ for a parabolic velocity profile and $\beta=1 / 2$ for a uniform velocity profile. Essentially, complete solutions for the Graetz-Nusselt problem found in the literature are a combination of the methods of Graetz and Lévêque [7].

There are special cases where the no-slip or the no-shear boundary condition does not hold. The effects of slip phenomena encountered in rarefied gas flows, which include velocity and temperature jumps at the tube wall $[4,9]$, were first investigated by Sparrow \& Lin [10]. Both wall slip and temperature jump are a function of the Knudsen number $K n$, the ratio of mean free path to tube diameter. Their results show that $N u_{\infty}$ decreases with increasing mean free path, which implies increasing wall slip and larger temperature jumps. Barron et al. [11] found that, for a given Graetz number, the Nusselt number becomes larger with increasing slip. Although they explicitly incorporated the temperature jump in the boundary conditions, it was ignored in the calculation of the eigenvalues [12]. It is crucial to account for the temperature jump at the wall for rarefied gas flows, since the effect of this temperature jump is dominant over the influence of wall slip $[12,13]$.

In continuum flows, i.e. liquid and gas flows for which $K n<10^{-2}$, a temperature jump is not present $[4,9]$. Moreover, in most situations the no-slip boundary condition is correct $[4,14]$. However, with the rise of micro- and nanofluidics it became apparent that the no-slip boundary condition for liquid flows does not always hold [14]. Intrinsic slip lengths vary from nearly zero [15, 16] to almost 
infinity $[17,18]$. Here, the slip length is defined by Navier's slip condition [19],

$$
u_{b}=-\left.b \frac{\partial u}{\partial r}\right|_{r=R},
$$

which states that the liquid velocity at the wall $u_{b}$ is proportional to the slip length $b$ and the velocity gradient $\partial_{r} u$ at the wall. This implies that the classical Graetz-Nusselt solutions are not applicable to this type of flow.

In this paper we present numerical and analytical solutions to the GraetzNusselt problem for continuum flows with finite slip. The effects of wall slip on both the exponent $\beta$ and $N u_{\infty}$, characteristic for heat transport in the thermally developing and thermally developed regimes, are investigated. The results reveal distinct transition points for $\beta$ and $N u_{\infty}$ when the slip length $b$ goes from zero to infinity. These transition points are explained both mathematically and physically.

\subsection{Mathematical model}

\subsubsection{Heat equation}

A schematic representation of the Graetz-Nusselt problem is provided in Fig. 6.1. Here, under the assumptions given in Sec. 6.1, the governing equation describing stationary heat transport in a cylindrical system can be written as

$$
u \frac{\partial T}{\partial x}=\frac{\alpha}{r} \frac{\partial}{\partial r}\left(r \frac{\partial T}{\partial r}\right),
$$

where $u(r, b)$ describes the velocity profile of the laminar fluid flow in the $x$ direction. The boundary conditions are $T(0, r)=T_{0}$ and $T(x, R)=T_{1}$. At $x=0$, the flow is hydrodynamically fully developed. Solution of the NavierStokes equation for stationary slip flow in the axial direction yields the following expression for the velocity profile:

$$
\tilde{u}=\frac{2\left(1-\tilde{r}^{2}\right)+4 \tilde{b}}{1+4 \tilde{b}},
$$

where $\tilde{u}=u / u_{a v}, \tilde{r}=r / R$, and $\tilde{b}=b / R$. Here, $\tilde{u}$ can be interpreted as the sum of the variable velocity $\tilde{u}_{v}(\tilde{r}, \tilde{b})=2\left(1-\tilde{r}^{2}\right) /(1+4 \tilde{b})$ and the slip velocity $\tilde{u}_{b}(\tilde{b})=4 \tilde{b} /(1+4 \tilde{b})$. The heat equation can now be non-dimensionalised using $\Theta=\left(T_{1}-T\right) /\left(T_{1}-T_{0}\right)$ and $\tilde{x}=x / L$,

$$
\frac{\left(1-\tilde{r}^{2}\right)+2 \tilde{b}}{2(1+4 \tilde{b})} \frac{\partial \Theta}{\partial(\tilde{x} / G z)}=\frac{1}{\tilde{r}} \frac{\partial}{\partial \tilde{r}}\left(\tilde{r} \frac{\partial \Theta}{\partial \tilde{r}}\right)
$$


with $\Theta(0, \tilde{r})=1$ and $\Theta(\tilde{x}, 1)=0$.

\subsubsection{Nusselt number}

Using Fourier's law of thermal conduction, the local heat transfer coefficient $h_{x}$ can be written as $h_{x}=-k /\left.\left(\langle T\rangle-T_{1}\right) \partial_{r} T\right|_{r=R}$ (see the appendix to this chapter for the details). By rewriting the temperature gradient in dimensionless form using the dimensionless flow-averaged temperature $\langle\Theta\rangle=\left(T_{1}-\langle T\rangle\right) /\left(T_{1}-T_{0}\right)$, we find for the local Nusselt number that

$$
N u_{x}=-\left.\frac{2}{\langle\Theta\rangle} \frac{\partial \Theta}{\partial \tilde{r}}\right|_{\tilde{r}=1} .
$$

When $\tilde{x} / G z>0.1, N u_{x} \rightarrow N u_{\infty}$.

\subsubsection{Analytical expressions for thermally developing flow}

To find an analytical expression for $N u_{x}$ near the entrance of the pipe, the Lévêque approximation is followed. In that case, the governing equation can be solved in a two-dimensional Cartesian coordinate system:

$$
u \frac{\partial T}{\partial x}=\alpha \frac{\partial^{2} T}{\partial y^{2}}
$$

Here, the wall is located at $y=0$, i.e. the direction of the $y$-axis is the reverse of that of the $r$-axis. Then, with $\tilde{y}=y / R$, the velocity profile becomes $\tilde{u}=$ $4(\tilde{y}+\tilde{b}) /(1+4 \tilde{b})$.

The analytical expressions for $N u_{x}$ for no-slip and no-shear flow are known [5], and the full mathematical and numerical details are given in the appendix. For $\tilde{b}=0$ one can derive that

$$
N u_{x}=\frac{2}{9^{\frac{1}{3}} \Gamma\left(\frac{4}{3}\right)}\left(\frac{\tilde{x}}{G z}\right)^{-\frac{1}{3}},
$$

where $\Gamma$ denotes the gamma function. For no-shear flow, i.e. $b=\infty$, one finds

$$
N u_{x}=\frac{1}{\sqrt{\pi}}\left(\frac{\tilde{x}}{G z}\right)^{-\frac{1}{2}}
$$

Thus, $\beta=1 / 3$ for parabolic flow and $\beta=1 / 2$ for uniform flow.

To arrive at an analytical expression for $N u_{x}$ for finite $\operatorname{slip}($ i.e. $0<\tilde{b}<\infty)$, Eq. (6.9) is non-dimensionalised using $Y=y / b$ and $X=x \alpha(R+4 b) /\left(4 u_{a v} b^{3}\right)=$ $(\tilde{x} / G z)(1+4 \tilde{b}) / \tilde{b}^{3}$. This gives

$$
(1+Y) \frac{\partial \Theta}{\partial X}=\frac{\partial^{2} \Theta}{\partial Y^{2}}
$$


with $\Theta(0, Y)=1, \Theta(X, 0)=0$ and $\Theta(X, \infty)=1$. To reduce the number of variables, we perform the Laplace transformation

$$
\mathcal{L}_{X}\left[\frac{\partial \Theta}{\partial X}\right]=\int_{0}^{\infty} \frac{\partial \Theta}{\partial X} e^{-p X} \mathrm{~d} X=p \bar{\Theta}(p, Y)-1,
$$

where $\bar{\Theta}$ is the Laplace transform of $\Theta$. Furthermore, $\mathcal{L}_{X}\left[\partial_{Y}^{2} \Theta\right]=\partial_{Y}^{2} \bar{\Theta}$. The governing equation now becomes

$$
(1+Y)(p \bar{\Theta}-1)=\frac{\partial^{2} \bar{\Theta}}{\partial Y^{2}}
$$

with $\bar{\Theta}(p, 0)=0$ and $\bar{\Theta}(p, \infty)=1 / p$. Introduction of $\hat{\Theta}=\bar{\Theta}-1 / p$ and subsequent rewriting yields

$$
p(1+Y) \hat{\Theta}=\frac{\partial^{2} \hat{\Theta}}{\partial Y^{2}}
$$

with $\hat{\Theta}(p, 0)=-1 / p$ and $\hat{\Theta}(p, \infty)=0$. To convert this expression into an ordinary differential equation (ODE), we define $\eta=p^{1 / 3}(1+Y)$. Now the Airy equation is found,

$$
\frac{\mathrm{d}^{2} \hat{\Theta}}{\mathrm{d} \eta^{2}}-\eta \hat{\Theta}=0
$$

with $\hat{\Theta}\left(p^{1 / 3}\right)=-1 / p$ and $\hat{\Theta}(\infty)=0$. Its general solution of the first kind is the Airy function $\operatorname{Ai}(\eta)$, with $\lim _{\eta \rightarrow \infty} \operatorname{Ai}(\eta)=0$. Then the solutions for $\hat{\Theta}$ and $\bar{\Theta}$ become

$$
\hat{\Theta}=-\frac{\operatorname{Ai}\left(p^{1 / 3}(1+Y)\right)}{p \operatorname{Ai}\left(p^{1 / 3}\right)} \text { and } \bar{\Theta}=\frac{\operatorname{Ai}\left(p^{1 / 3}\right)-\operatorname{Ai}\left(p^{1 / 3}(1+Y)\right)}{p \operatorname{Ai}\left(p^{1 / 3}\right)} .
$$

To obtain $\Theta$, we take the inverse Laplace transform in $X$, giving

$$
\Theta(X, Y)=\frac{1}{2 \pi \mathrm{i}} \int_{c-\mathrm{i} \infty}^{c+\mathrm{i} \infty} \frac{\operatorname{Ai}\left(p^{1 / 3}\right)-\operatorname{Ai}\left(p^{1 / 3}(1+Y)\right)}{p \operatorname{Ai}\left(p^{1 / 3}\right)} e^{p X} \mathrm{~d} p .
$$

For $N u_{x}$ we can derive that, given that $\langle\Theta\rangle=1$ following the Lévêque approximation,

$$
N u_{x}=\left.\frac{2}{\tilde{b}} \frac{\partial \Theta}{\partial Y}\right|_{Y=0}
$$

Then, finally, we obtain

$$
N u_{x}=-\frac{1}{\tilde{b} \pi \mathrm{i}} \int_{c-\mathrm{i} \infty}^{c+\mathrm{i} \infty} \frac{\operatorname{Ai}^{\prime}\left(p^{1 / 3}\right) e^{p X}}{p^{2 / 3} \operatorname{Ai}\left(p^{1 / 3}\right)} \mathrm{d} p=\frac{2}{\tilde{b}} g(X) .
$$

The function $g(X)$ is universal, as it does not depend on $\tilde{b}$. The slip length affects 
the scaling between $X$ and $\tilde{x} / G z$, so it determines which part of the function $g(X)$ is relevant. The appendix to this chapter provides a description of how to evaluate $g(X)$.

\subsubsection{Thermal and viscous boundary layer thickness}

The thermal boundary layer thickness $\tilde{\lambda}_{T}$ is defined as $\tilde{\lambda}_{T}=1-\tilde{r}(\Theta=0.99)$. When $\Theta(\tilde{x} / G z, 0)<0.99, \tilde{\lambda}_{T}=1$.

The viscous boundary layer $\tilde{\delta}_{\nu}$ is defined as follows. It corresponds to the point $\tilde{r}=1-\tilde{\delta}_{\nu}$ where the parabolic velocity component $\tilde{u}_{v}(\tilde{r})$ equals $c$ times the slip velocity $\tilde{u}_{b}$ at the wall. Thus, $\tilde{\delta}_{\nu}\left(\tilde{u}_{v}=c \tilde{u}_{b}\right)=1-(1-2 c \tilde{b})^{1 / 2}$. When $\tilde{b} \geq 1 /(2 c)$, $\tilde{\delta}_{\nu}=1$. It should be noted that, for a given $\tilde{b}$ and $c, \tilde{\delta}_{\nu}$ is fixed for all $\tilde{x} / G z$, while $\tilde{\lambda}_{T}=f(\tilde{x} / G z)$.

\subsubsection{Numerical procedure}

Numerically, the Graetz-Nusselt problem for finite slip was solved using the pdepe-solver in MATLAB (MathWorks). The relative and absolute tolerances for the pdepe-solver were set at $10^{-6}$ and $10^{-12}$ respectively. To calculate $\partial_{\tilde{r}} \Theta$ at $\tilde{r}=1$ the MATLAB function pdeval was utilised.

Because heat transport mainly occurs near the inlet and near the wall, the $\tilde{x} \times \tilde{r}=82 \times 101$ mesh was refined near these boundaries. The exponent $\beta$ was evaluated in two distinct manners. The fitting of a straight line through $\log _{10}\left(N u_{x}\right)$ for $-7 \leq \log _{10}(\tilde{x} / G z) \leq-4$ using the polyfit algorithm gives a specific constant value for $\beta$ for each $\tilde{b}$, which is referred to as $\beta_{f}$. The gradient of $\log _{10}\left(N u_{x}\right)$ or $\log _{10}(g(X))$ to $\log _{10}(\tilde{x} / G z)$, calculated by employing the second-order-accurate gradient algorithm, gives a local value for $\beta$ for each position, which is referred to as $\beta_{l}$. Here, $N u_{\infty}$ is taken as the average of $N u_{x}$ for $\tilde{x} / G z \geq 0.1$. To compute $\langle\Theta\rangle$ the trapz script was utilised. The value of $\tilde{\lambda}_{T}$ was approximated by linear interpolation using the two grid points closest to $\Theta=0.99$.

\subsection{Results and discussion}

\subsubsection{Nusselt profiles}

In Fig. 6.2( $a)$ the Nusselt profiles are displayed for a wide range of slip lengths $\tilde{b}$. The profiles reveal that for $\tilde{b}<10^{-4}$ the classical Graetz-Nusselt solution for no-slip flow is approached, with $\beta=1 / 3$. For $\tilde{b}>10^{2}$ the classical solution for no-shear flow is recovered, for which $\beta=1 / 2$. For intermediate values of $\tilde{b}$, the figure shows that first the exponent $\beta$ (the slope) starts to change value, followed by an increase of $N u_{\infty}$.

The dependence of $\beta_{f}$ and $N u_{\infty}$ on the slip length $\tilde{b}$ is shown in Fig. $6.2(b)$. This figure illustrates that the transition points for $\beta_{f}$ and $N u_{\infty}$ are located 
(a)
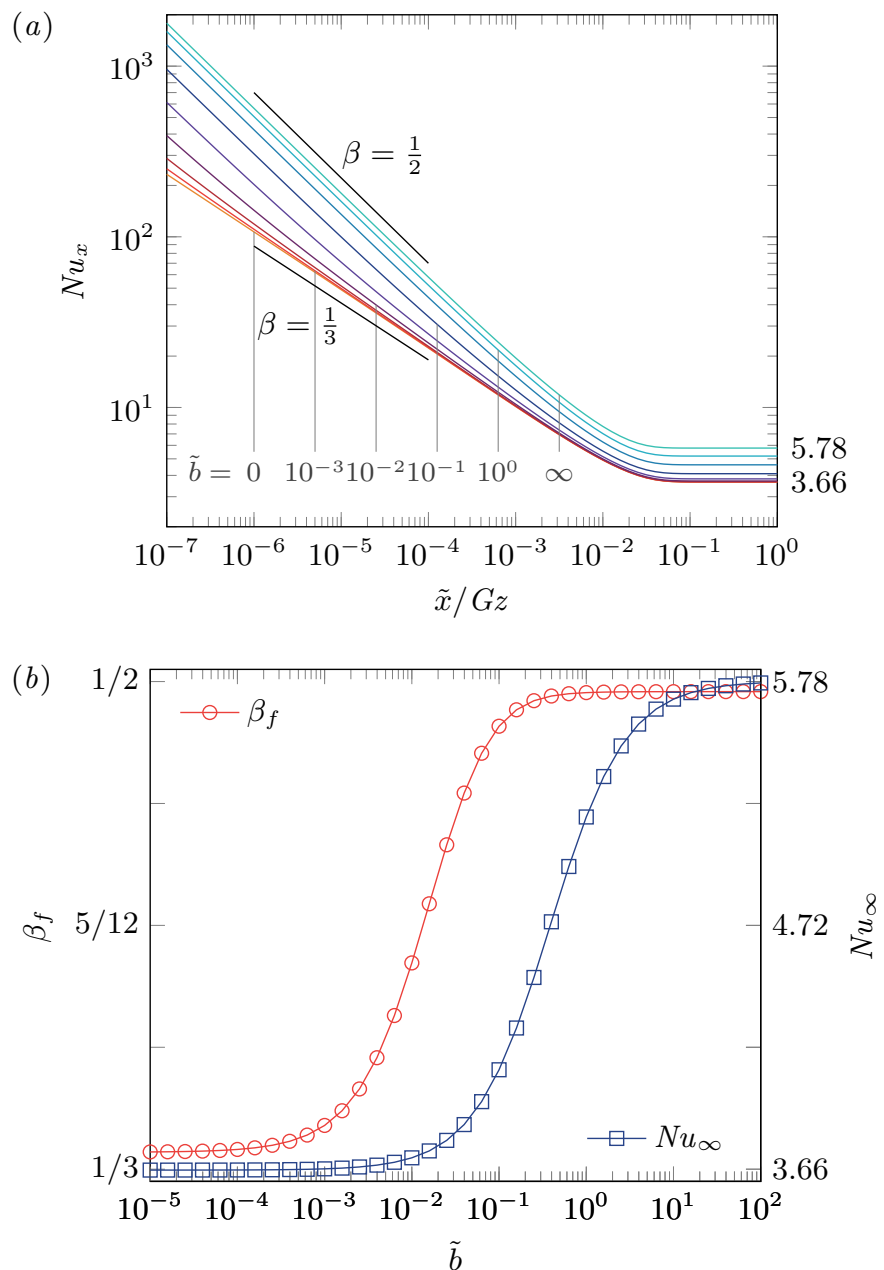

Figure 6.2 - (a) Local Nusselt number $N u_{x}$ as a function of the position $\tilde{x} / G z$ for various slip lengths $\tilde{b}$. The values of the scaling exponent $\beta$ and the developed Nusselt number $N u_{\infty}$ for the limiting cases of no-slip $(\tilde{b}=0)$ and no-shear flow $(\tilde{b}=\infty)$ are also indicated. (b) Scaling exponent $\beta_{f}$ (obtained by fitting) and developed Nusselt number $N u_{\infty}$ as a function of the slip length $\tilde{b}$. Remarkably, these two curves transition at different $\tilde{b}$.

more than one order of magnitude apart. The exponent $\beta_{f}$ changes value when $10^{-4}<\tilde{b}<10^{0}$, while $N u_{\infty}$ gradually increases from 3.66 to 5.78 when $10^{-2}<$ $\tilde{b}<10^{2}$. This corresponds to the values for $N u_{\infty}$ found by Barron et al. [11], who solved the Graetz-Nusselt problem in the thermally developed regime for $0 \leq \tilde{b} \leq 0.24$ [12]. It should be noted that a change in the range of $\tilde{x} / G z$ values used to compute $\beta_{f}$ results in a different transition point for $\beta_{f}$. The use of larger 
values shifts the transition point upwards. Nonetheless, the distance between the transition points for $\beta_{f}$ and $N u_{\infty}$ remains of at least one order of magnitude large.

The exponent $\beta$ is not necessarily a specific constant value for finite slip as it is for no-slip and no-shear flow. Therefore the local exponent $\beta_{l}$ was evaluated both numerically and analytically. In Fig. $6.3(a), \beta_{l}$ is plotted versus $\tilde{x} / G z$ for various slip lengths $\tilde{b}$. The profiles confirm that for approximately $10^{-4}<\tilde{b}<10^{0}, \beta_{l}$ is not constant and depends on both the position and the slip length. Furthermore, we observe that $\beta_{l}$ always transitions from $1 / 2$ to $1 / 3$, except for the limits $\tilde{b}=0$ and $\tilde{b}=\infty$.

The numerical and analytical results are in good agreement, but start to deviate when $\tilde{x} / G z>10^{-5}$. In that case the thermal and viscous boundary layer thicknesses are of the same order of magnitude $\left(\tilde{\lambda}_{T} \approx 0.1\right.$ for $\left.\tilde{x} / G z=10^{-4}\right)$. Consequently, the assumptions arising from the Lévêque approximation, and therefore also the analytical results, are no longer entirely valid.

\subsubsection{On $\beta$ and its transition point}

That $\beta_{l}$ must transition from $1 / 2$ to $1 / 3$, except for $\tilde{b}=0$ and $\tilde{b}=\infty$, can be demonstrated by inspection of the function $g(X)$. First, as Fig. 6.3(b) reveals, the classical limits are recovered by this function, as $N u_{x} \propto g(X)$. When $\tilde{b} \rightarrow 0$, we would expect that $g(X) \propto X^{-1 / 3}$ for $X \rightarrow \infty$. Thus

$$
N u_{x} \propto \frac{1}{\tilde{b}}\left(\frac{\tilde{x}}{G z} \frac{1}{\tilde{b}^{3}}\right)^{-1 / 3}=\left(\frac{\tilde{x}}{G z}\right)^{-1 / 3} .
$$

When $\tilde{b} \rightarrow \infty$, we would expect that for $X \rightarrow 0, g(X) \propto X^{-1 / 2}$. Then

$$
N u_{x} \propto \frac{1}{\tilde{b}}\left(\frac{\tilde{x}}{G z} \frac{\tilde{b}}{\tilde{b}^{3}}\right)^{-1 / 2}=\left(\frac{\tilde{x}}{G z}\right)^{-1 / 2} .
$$

Second, for each value of $0<\tilde{b}<\infty$ we can choose $\tilde{x}$ such that $X \rightarrow 0$. As in that case Eq. (6.22) is recovered, we find that for finite slip $\beta_{l}$ always transitions from $1 / 2$ to $1 / 3$. Consequently, it can be concluded that for a given slip length $\tilde{b}$, the Nusselt profile in the thermally developing regime cannot be characterised by a single $\beta_{f}$ value. The range of $\tilde{x} / G z$-values used for computing $\beta_{f}$ is determinant for the value of $\beta_{f}$ that is obtained. Nevertheless, comparison of Fig. 6.2(b) and $6.3(a)$ shows that $\beta_{f}$ does reflect for what range of slip lengths $\tilde{b}$ the exponent $\beta$ changes value. Finally, since $\beta_{l} \approx 5 / 12$ when $X=1$, the dimensionless number $X=(\tilde{x} / G z)(1+4 \tilde{b}) / \tilde{b}^{3}$ can be considered as a kind of criterion for the behaviour of $\beta$ : $\beta \rightarrow 1 / 2$ when $X \ll 1$, whereas $\beta \rightarrow 1 / 3$ when $X \gg 1$.

Physically, the location of the transition regime for $\beta_{l}$ can be explained by 

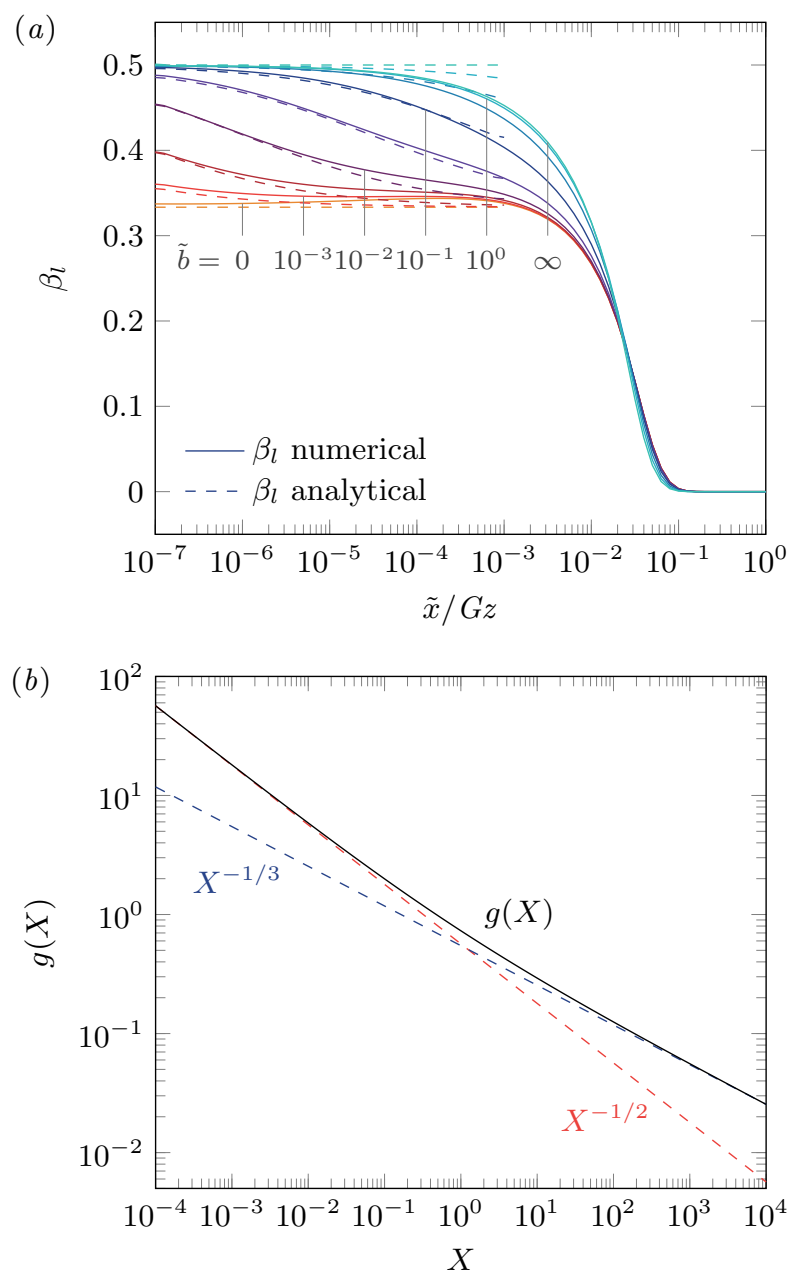

Figure 6.3 - (a) Local scaling exponent $\beta_{l}$ versus position $\tilde{x} / G z$ for various slip lengths $\tilde{b}$. (b) The limiting behaviour of $g(X)$, from which $\beta_{l}$ can be obtained analytically, is as expected: $g(X) \propto X^{-1 / 2}$ when $X \rightarrow 0$, and $g(X) \propto X^{-1 / 3}$ when $X \rightarrow \infty$.

considering the thickness of the thermal and viscous boundary layers. This is illustrated in Fig. 6.4(a). All heat transport takes place in the thermal boundary layer, where the temperature gradient at the wall, $\left.\partial_{\tilde{r}} \Theta\right|_{\tilde{r}=1}$, is most important. When the velocity in the thermal boundary layer, which is growing with $\tilde{x}$, is dominated by the slip velocity $\tilde{u}_{b}, \beta_{l} \rightarrow 1 / 2$. In that case, the velocity profile in the thermal boundary layer is approximately uniform, and thereby resembles a no-shear flow. Then the left-hand side of Eq. (6.9) can be approximated as $u \partial_{x} T=u_{v} \partial_{x} T+u_{b} \partial_{x} T \approx u_{b} \partial_{x} T$. On the other hand, when the velocity in the 
$(a)$

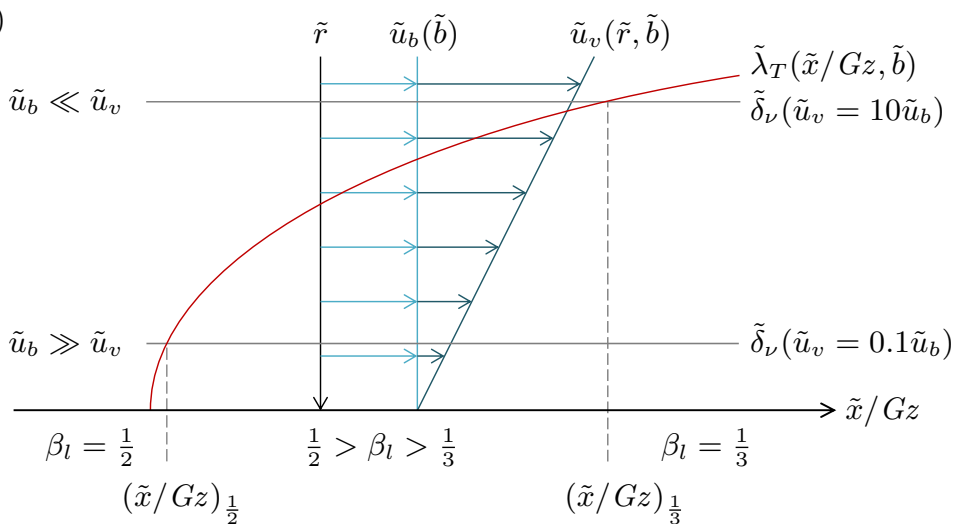

(b)

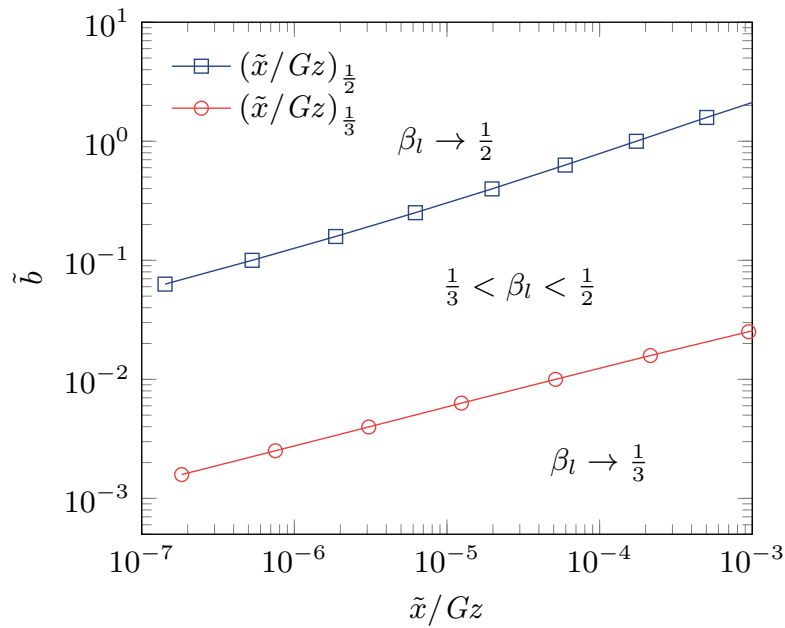

Figure 6.4 - (a) The location of the transition regime for $\beta_{l}$ can be explained physically by considering $\tilde{\lambda}_{T}$ and $\tilde{\delta}_{\nu}$. When the slip velocity $\tilde{u}_{b}$ dominates in the thermal boundary layer, which is the case when $\tilde{x} / G z<(\tilde{x} / G z)_{1 / 2}, \beta_{l} \rightarrow 1 / 2$. When the velocity in the thermal boundary layer is dominated by $\tilde{u}_{v}, \beta_{l} \rightarrow 1 / 3$. This is true when $\tilde{x} / G z>(\tilde{x} / G z)_{1 / 3}$. (b) The locations of $(\tilde{x} / G z)_{1 / 2}$ and $(\tilde{x} / G z)_{1 / 3}$ as a function of $\tilde{b}$ are shown here. These points are estimated by saying that $\tilde{u}_{v} \ll \tilde{u}_{b}$ when $\tilde{u}_{v}=0.1 \tilde{u}_{b}$, and that $\tilde{u}_{v} \gg \tilde{u}_{b}$ when $\tilde{u}_{v}=10 \tilde{u}_{b}$.

thermal boundary layer is dominated by the parabolic velocity component $\tilde{u}_{v}$, $\beta_{l} \rightarrow 1 / 3$. The flow profile in the thermal boundary layer resembles a no-slip flow, i.e. $u \partial_{x} T \approx u_{v} \partial_{x} T$, for which $\beta=1 / 3$.

The location where $\beta_{l}$ transitions from $1 / 2$ to $1 / 3$ can be predicted. In the thermal boundary layer, the slip velocity $\tilde{u}_{b}$ is dominating $\left(i . e . \tilde{u}_{v} \ll \tilde{u}_{b}\right)$ until the 
point $(\tilde{x} / G z)_{1 / 2}$. This location is estimated by calculating where $\tilde{\lambda}_{T}=\tilde{\delta}_{\nu}\left(\tilde{u}_{v}=\right.$ $\left.0.1 \tilde{u}_{b}\right)$. The parabolic velocity component starts to dominate in the thermal boundary layer (i.e. $\left.\tilde{u}_{v} \gg \tilde{u}_{b}\right)$ from the point $(\tilde{x} / G z)_{1 / 3}$ on. The point where $\tilde{\lambda}_{T}=\tilde{\delta}_{\nu}\left(\tilde{u}_{v}=10 \tilde{u}_{b}\right)$ roughly corresponds to this location. This implies that the transition regime for $\beta_{l}$ is located between $(\tilde{x} / G z)_{1 / 2}$ and $(\tilde{x} / G z)_{1 / 3}$. The locations of $(\tilde{x} / G z)_{1 / 2}$ and $(\tilde{x} / G z)_{1 / 3}$ are given in Fig. $6.4(b)$. As an example, for $\tilde{b}=10^{-1}$ we find that $(\tilde{x} / G z)_{1 / 2}=5 \times 10^{-7}$. This is in agreement with $\beta_{l}$ for $\tilde{b}=10^{-1}$ in Fig. 6.3(a).

\subsubsection{On $N u_{\infty}$ and its transition point}

The developed Nusselt number $N u_{\infty}$ concerns heat transfer in the thermally developed regime $(\tilde{x} / G z>0.1)$. In this regime the temperature and temperature gradient at the wall are approaching zero. This implies that $N u_{\infty}$ can only be enhanced by increasing advective transport near the wall, where the temperature gradients are largest. Fig. 6.5 demonstrates that when $\tilde{u}_{b}$ starts to change significantly with $\tilde{b}, N u_{\infty}$ also transitions from 3.66 to 5.78 , the limiting values for zero and infinite slip length.

Replacement of $\tilde{u}_{b}$ by the velocity gradient at the wall, $\left.\partial_{\tilde{r}} \tilde{u}\right|_{\tilde{r}=1}$, leads to the same conclusion, however. An increase in $\tilde{u}_{b}$ implies that $\left|\partial_{\tilde{r}} \tilde{u}\right|$ must decrease. This follows from Navier's slip condition, $\tilde{u}_{b}=-\left.\tilde{b} \partial_{\tilde{r}} \tilde{u}\right|_{\tilde{r}=1}$, which shows that the slip velocity and the velocity gradient at the wall are directly related to each other via the factor $-\tilde{b}$. Moreover, from the definitions of $\tilde{u}_{b}=4 \tilde{b} /(1+4 \tilde{b})$ and $\left.\partial_{\tilde{r}} \tilde{u}\right|_{\tilde{r}=1}=-4 /(1+4 \tilde{b})$, we find that they have the same transition point, which is $\tilde{b}=1 / 4$. This is close to the transition point of $\tilde{b}=0.4$ for $N u_{\infty}$. Furthermore, their derivatives to $\tilde{b}, \partial_{\tilde{b}} \tilde{u}_{b}$ and $\left.\partial_{\tilde{b}}\left(\partial_{\tilde{r}} \tilde{u}\right)\right|_{\tilde{r}=1}$, are both proportional to $(1+4 \tilde{b})^{-2}$.

Therefore, the change of neither $\tilde{u}_{b}$ nor $\left.\partial_{\tilde{r}} \tilde{u}\right|_{\tilde{r}=1}$ with slip length $\tilde{b}$ fully explains the dependence of $N u_{\infty}$ on $\tilde{b}$. It is the relation between the velocity distribution and the slip length $\tilde{b}$ that explains how $N u_{\infty}$ depends on $\tilde{b}$. Nevertheless, because heat transport is largest when temperature gradients are maximum, the change of $\tilde{u}_{b}$ with $\tilde{b}$ most dominantly affects the behaviour of $N u_{\infty}$. This is in agreement with the observation of Barrow \& Humphreys [20] that, given an average fluid velocity, an increase of the velocity at the wall leads to larger Nusselt numbers.

\subsubsection{Employing wall slip to increase heat transfer}

The Nusselt profiles in Fig. 6.2(a) suggest that heat transport can be optimised by employing wall slip, provided that the slip length is large enough. Partial slip lengths are usually of the order of tens of nanometres for microscale systems [16]. As a consequence, the maximum tube radius is about $1 \mu \mathrm{m}$ for $\beta$ to be significantly larger than $1 / 3$. For $N u_{\infty}$ to be larger than 3.66 , tube radii should be of the order of nanometres. However, the Graetz number is usually very small. For a typical liquid like water, $R e \sim 1$ and $\operatorname{Pr} \sim 1$. For both micro- and nanofluidics 


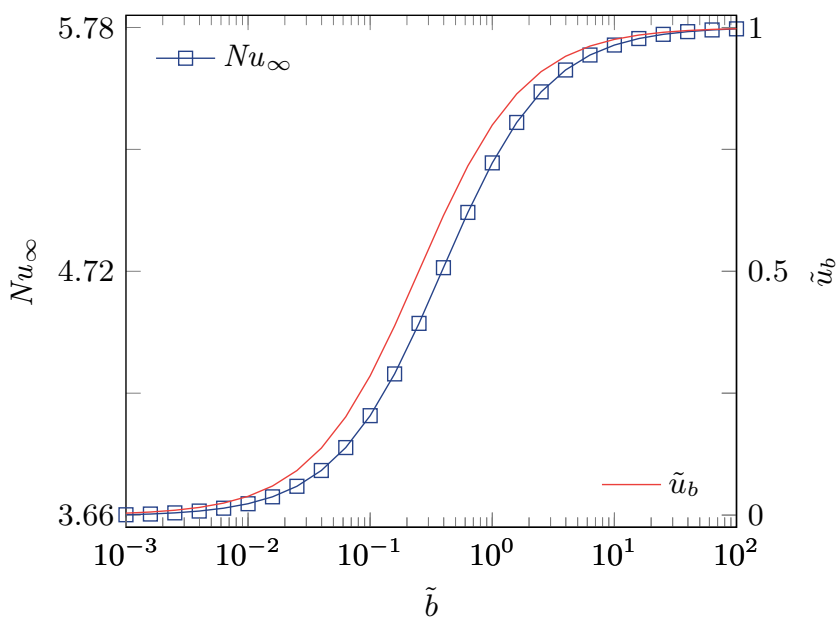

Figure 6.5 - Developed Nusselt number $N u_{\infty}$ and slip velocity $\tilde{u}_{b}$ versus slip length $\tilde{b}$.

it is estimated that $D / L<10^{-3}$, implying that $G z<10^{-3}$. In that case, the temperature profile is almost instantaneously thermally developed, as this is then true for $\tilde{x}>10^{-4}$.

Thus, enhancement of heat transfer by employing finite slip, while avoiding reaching the thermally developed regime, is possible if two conditions are met. First, the system should be designed such that $G z>1$. Second, the slip length should be of the order of the tube radius, i.e. $\tilde{b} \sim 1$. These conditions oppose one another. In nanofluidic systems, slip lengths $\tilde{b}$ of about $10^{3}$ can be obtained $[17,18]$. This implies that $\tilde{b}$ is large enough to let $N u_{\infty} \rightarrow 5.78$, albeit the thermally developing section is negligibly small. Additionally, for instance, highly slippery liquid-infused porous surfaces [21,22] could be exploited to enhance heat transport.

It should be noted that the results presented here are applicable to systems that display homogeneous wall slip. Distinct results are expected for systems concerning effective slip [23-25]. As such, the Graetz-Nusselt problem for heterogeneously slippery surfaces deserves to be studied separately.

\subsection{Conclusion}

This study provides numerical and analytical solutions to the Graetz-Nusselt problem for continuum flows with finite slip. The classical solutions to this problem concern no-slip and no-shear flow. These form the lower and upper 
limits of the solutions for partial slip, as the resulting Nusselt profiles gradually transition between these limits on increasing the wall slip from zero to infinity.

The heat transfer mechanism in the thermally developing regime depends on the velocity profile in the thermal boundary layer, and is a function of wall slip and position. The ratio of the parabolic velocity component to the slip velocity determines whether it resembles the transport mechanism for no-slip or for noshear flow. The Nusselt number in the thermally developed regime depends on the fluid velocity profile only.

By considering slip lengths ranging from zero to infinity, this study is the first to connect the classical solutions to the Graetz-Nusselt problem. This is not only of fundamental interest, but also makes it possible to evaluate the influence of wall slip on heat transfer in many forced convection problems in science and engineering.

\section{References}

[1] L. Graetz, Über die Wärmeleitungsfähigkeit von Flüssigkeiten, Ann. Phys. 254, 79 (1882).

[2] L. Graetz, Über die Wärmeleitungsfähigkeit von Flüssigkeiten, Ann. Phys. 261, 337 (1885).

[3] W. Nusselt, Die Abhängigkeit der Wärmeübergangszahl von der Rohrlänge, Z. Ver. Deut. Ing. 54, 1154 (1910).

[4] E. R. G. Eckert and R. M. Drake, Analysis of heat and mass transfer (McGraw-Hill Kogakusha, Tokyo, 1972).

[5] R. B. Bird, W. E. Stewart, and E. N. Lightfoot, Transport phenomena, 2nd ed. (John Wiley \& Sons, New York, 2007).

[6] M. Jakob, Heat transfer (John Wiley \& Sons, New York, 1949), Vol. 1.

[7] R. K. Shah and A. L. London, Laminar flow forced convection in ducts: a source book for compact heat exchanger analytical data (Academic Press, New York, 1978).

[8] M. A. Lévêque, Les lois de la transmission de chaleur par convection, Ann. Mines, Mem., Ser. 13, 201 (1928).

[9] G. Karniadakis, A. Beskok, and N. Aluru, Microflows and nanoflows: fundamentals and simulation (Springer, New York, 2005).

[10] E. M. Sparrow and S. H. Lin, Laminar heat transfer in tubes under slip-flow conditions, J. Heat Transfer 84, 363 (1962).

[11] R. F. Barron, X. Wang, T. A. Ameel, and R. O. Warrington, The Graetz problem extended to slip-flow, Int. J. Heat Mass Transfer 40, 1817 (1997).

[12] F. Ezquerra Larrodé, C. Housiadas, and Y. Drossinos, Slip-flow heat transfer in circular tubes, Int. J. Heat Mass Transfer 43, 2669 (2000). 
[13] S. Colin, Gas microflows in the slip flow regime: A critical review on convective heat transfer, J. Heat Transfer 134, 020908 (2011).

[14] E. Lauga, M. P. Brenner, and H. A. Stone, in Springer handbook of experimental fluid mechanics, edited by C. Tropea, A. L. Yarin, and J. F. Foss (Springer, Berlin, 2007), pp. 1219-1240.

[15] L. Bocquet and J.-L. Barrat, Flow boundary conditions from nano- to microscales, Soft Matter 3, 685 (2007).

[16] J. P. Rothstein, Slip on superhydrophobic surfaces, Annu. Rev. Fluid Mech. 42, 89 (2010).

[17] M. Whitby and N. Quirke, Fluid flow in carbon nanotubes and nanopipes, Nat. Nano 2, 87 (2007).

[18] M. Majumder, N. Chopra, and B. J. Hinds, Mass transport through carbon nanotube membranes in three different regimes: Ionic diffusion and gas and liquid flow, ACS Nano 5, 3867 (2011).

[19] C. L. M. H. Navier, Mémoire sur les lois du mouvement des fluids, Mem. Acad. Sci. Inst. Fr. 6, 389 (1823).

[20] H. Barrow and J. F. Humphreys, The effect of velocity distribution on forced convection laminar flow heat transfer in a pipe at constant wall temperature, Wärme Stoffübertrag. 3, 227 (1970).

[21] T.-S. Wong, S. H. Kang, S. K. Y. Tang, E. J. Smythe, B. D. Hatton, A. Grinthal, and J. Aizenberg, Bioinspired self-repairing slippery surfaces with pressure-stable omniphobicity, Nature 477, 443 (2011).

[22] A. Lafuma and D. Quéré, Slippery pre-suffused surfaces, EPL (Europhys. Lett.) 96, 56001 (2011).

[23] D. Maynes, B. W. Webb, and J. Davies, Thermal transport in a microchannel exhibiting ultrahydrophobic microribs maintained at constant temperature, J. Heat Transfer 130, 022402 (2008).

[24] D. Maynes, B. W. Webb, J. Crockett, and V. Solovjov, Analysis of laminar slip-flow thermal transport in microchannels with transverse rib and cavity structured superhydrophobic walls at constant heat flux, J. Heat Transfer 135, 021701 (2012).

[25] R. Enright, M. Hodes, T. Salamon, and Y. Muzychka, Isoflux Nusselt number and slip length formulae for superhydrophobic microchannels, J. Heat Transfer 136, 012402 (2013). 


\section{Appendix}

\section{Nusselt number}

The local Nusselt number $N u_{x}$ is defined in Eq. (6.1) as

$$
N u_{x}=\frac{h_{x} D}{k}
$$

Following Newton's law of cooling and using Fourier's law of thermal conduction, the heat transfer coefficient $h_{x}$ can be rewritten as

$$
h_{x}=\frac{q "}{\Delta T}=-\left.\frac{k}{\langle T\rangle-T_{1}} \frac{\partial T}{\partial r}\right|_{r=R},
$$

where $q$ " represents the local heat flux between the wall and the fluid. Rewriting the temperature gradient in dimensionless form gives

$$
h_{x}=-\left.\frac{2 k}{D} \frac{1}{\langle\Theta\rangle} \frac{\partial \Theta}{\partial \tilde{r}}\right|_{\tilde{r}=1},
$$

where $\langle\Theta\rangle=\left(T_{1}-\langle T\rangle\right) /\left(T_{1}-T_{0}\right)$. Here, it is always true that $\partial_{\tilde{r}} \Theta \leq 0$. Now we obtain Eq. (6.8) for $N u_{x}$ :

$$
N u_{x}=-\left.\frac{2}{\langle\Theta\rangle} \frac{\partial \Theta}{\partial \tilde{r}}\right|_{\tilde{r}=1}
$$

The dimensionless flow-averaged or mixing-cup temperature $\langle\Theta\rangle$, which is a function of the local Graetz number $(\tilde{x} / G z)$, is obtained by the following integral:

$$
\langle\Theta\rangle=\int_{0}^{1} \Theta \tilde{u} \tilde{r} \mathrm{~d} \tilde{r} / \int_{0}^{1} \tilde{u} \tilde{r} \mathrm{~d} \tilde{r} .
$$

\section{Analytical solutions in the thermally developing regime}

No-slip flow. Following the Lévêque approximation, in the case of no-slip flow the velocity profile near the wall can be written as $\tilde{u}=4 \tilde{y}$. When making Eq. (6.9) dimensionless, the following governing equation is obtained for no-slip flow:

$$
G z \tilde{y} \frac{\partial \Theta}{\partial \tilde{x}}=\frac{\partial^{2} \Theta}{\partial \tilde{y}^{2}},
$$

with $\Theta(0, \tilde{y})=1, \Theta(\tilde{x}, 0)=0$, and $\Theta(\tilde{x}, \infty)=1$. By introducing a similarity variable $\eta$, where

$$
\eta=\left(\frac{G z \tilde{y}^{3}}{9 \tilde{x}}\right)^{\frac{1}{3}}
$$

and subsequent rewriting of the energy balance, we obtain the following ordinary 
differential equation (ODE):

$$
\frac{\mathrm{d}^{2} \Theta}{\mathrm{d} \eta^{2}}+3 \eta^{2} \frac{\mathrm{d} \Theta}{\mathrm{d} \eta}=0
$$

with boundary conditions $\Theta(0)=0$ and $\Theta(\infty)=1$. This ODE has a known solution, and with the boundary conditions given the final solution for $\Theta$ becomes

$$
\Theta=\frac{1}{\Gamma\left(\frac{4}{3}\right)} \int_{0}^{\eta} \exp \left(-\bar{\eta}^{3}\right) \mathrm{d} \bar{\eta} .
$$

Previously, an expression for the Nusselt number was found using the dimensionless temperature gradient $\partial_{\tilde{r}} \Theta$. Rewriting this in terms of $\partial_{\tilde{y}} \Theta$, the expression for $N u$ becomes

$$
N u_{x}=\left.\frac{2}{\langle\Theta\rangle} \frac{\partial \Theta}{\partial \tilde{y}}\right|_{\tilde{y}=0} .
$$

However, in the Lévêque approximation $\langle\Theta\rangle=1$. Furthermore, the dimensionless temperature gradient is rewritten in terms of $\mathrm{d}_{\eta} \Theta$. Then

$$
N u_{x}=\left.\frac{2 \eta}{\tilde{y}} \frac{\mathrm{d} \Theta}{\mathrm{d} \eta}\right|_{\eta=0} .
$$

The temperature gradient is always larger than zero, i.e. $\mathrm{d}_{\eta} \Theta>0$. Now, using the Leibniz formula for differentiation of integrals, an expression for the temperature gradient can be found:

$$
\frac{\mathrm{d} \Theta}{\mathrm{d} \eta}=\frac{\exp \left(-\eta^{3}\right)}{\Gamma\left(\frac{4}{3}\right)}
$$

Evaluation at $\eta=0$ and subsequent substitution in the expression for $N u_{x}$ yields

$$
N u_{x}=\frac{2}{\Gamma\left(\frac{4}{3}\right)} \frac{\eta}{\tilde{y}}
$$

or, as earlier presented in Eq. (6.10),

$$
N u_{x}=\frac{2}{9^{\frac{1}{3}} \Gamma\left(\frac{4}{3}\right)}\left(\frac{\tilde{x}}{G z}\right)^{-\frac{1}{3}} .
$$

No-shear flow. In the case of no-shear flow, the velocity profile is uniform: $\tilde{u}=1$. By rewriting Eq. (6.9), we obtain following governing equation for no-shear flow:

$$
\frac{G z}{4} \frac{\partial \Theta}{\partial \tilde{x}}=\frac{\partial^{2} \Theta}{\partial \tilde{y}^{2}},
$$


with $\Theta(0, \tilde{y})=1, \Theta(\tilde{x}, 0)=0$, and $\Theta(\tilde{x}, \infty)=1$. By using the similarity variable

$$
\eta=\left(\frac{G z \tilde{y}^{2}}{16 \tilde{x}}\right)^{\frac{1}{2}}
$$

we arrive at the following ODE:

$$
\frac{\mathrm{d}^{2} \Theta}{\mathrm{d} \eta^{2}}+2 \eta \frac{\mathrm{d} \Theta}{\mathrm{d} \eta}=0
$$

with $\Theta(0)=0$ and $\Theta(\infty)=1$. Solving this ODE gives

$$
\Theta=\frac{2}{\sqrt{\pi}} \int_{0}^{\eta} \exp \left(-\bar{\eta}^{2}\right) \mathrm{d} \bar{\eta}=\operatorname{erf}(\eta)
$$

Since

$$
\frac{\mathrm{d} \Theta}{\mathrm{d} \eta}=\frac{2}{\sqrt{\pi}} \exp \left(-\eta^{2}\right)
$$

this ultimately yields Eq. (6.11) for $N u_{x}$ :

$$
N u_{x}=\frac{1}{\sqrt{\pi}}\left(\frac{\tilde{x}}{G z}\right)^{-\frac{1}{2}}
$$

Slip flow. In Sec. 6.2.3, we derived that for flow with finite wall slip,

$$
N u_{x}=\left.\frac{2}{\tilde{b}} \frac{\partial \Theta}{\partial Y}\right|_{Y=0}=\frac{2}{\tilde{b}} g(X)
$$

From Eq. (6.18) for the dimensionless temperature $\Theta$, which equals

$$
\Theta(X, Y)=\frac{1}{2 \pi \mathrm{i}} \int_{c-\mathrm{i} \infty}^{c+\mathrm{i} \infty} \frac{\operatorname{Ai}\left(p^{1 / 3}\right)-\operatorname{Ai}\left(p^{1 / 3}(1+Y)\right)}{p \operatorname{Ai}\left(p^{1 / 3}\right)} e^{p X} \mathrm{~d} p
$$

and following Leibniz' integral rule, we find that

$$
\frac{\partial \Theta}{\partial Y}=-\frac{1}{2 \pi \mathrm{i}} \int_{c-\mathrm{i} \infty}^{c+\mathrm{i} \infty} \frac{\operatorname{Ai}^{\prime}\left(p^{1 / 3}(1+Y)\right) e^{p X}}{p^{2 / 3} \operatorname{Ai}\left(p^{1 / 3}\right)} \mathrm{d} p .
$$

The function $g(X)$ therefore equals

$$
g(X)=-\frac{1}{2 \pi \mathrm{i}} \int_{c-\mathrm{i} \infty}^{c+\mathrm{i} \infty} \frac{\mathrm{Ai}^{\prime}\left(p^{1 / 3}\right) e^{p X}}{p^{2 / 3} \operatorname{Ai}\left(p^{1 / 3}\right)} \mathrm{d} p,
$$

with $X$ being

$$
X=\left(\frac{\tilde{x}}{G z}\right) \frac{1+4 \tilde{b}}{\tilde{b}^{3}}
$$




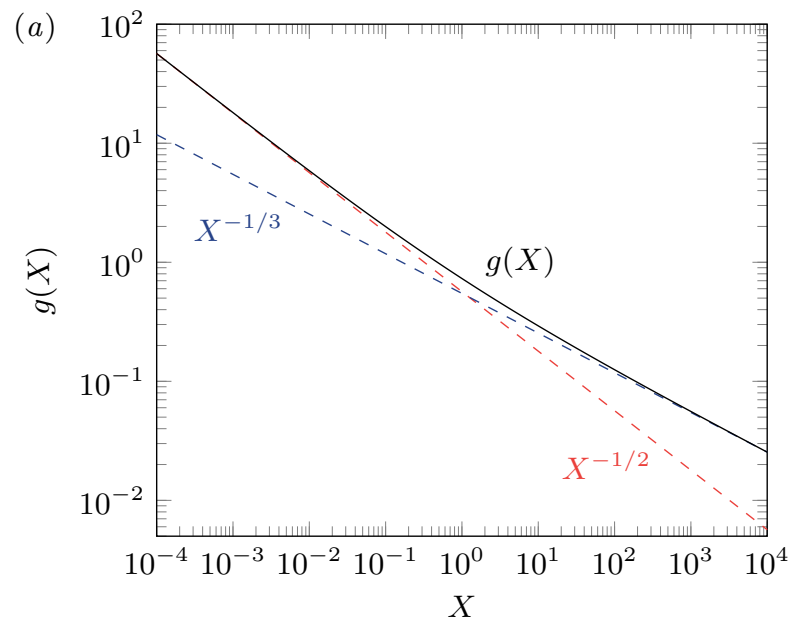

(b)

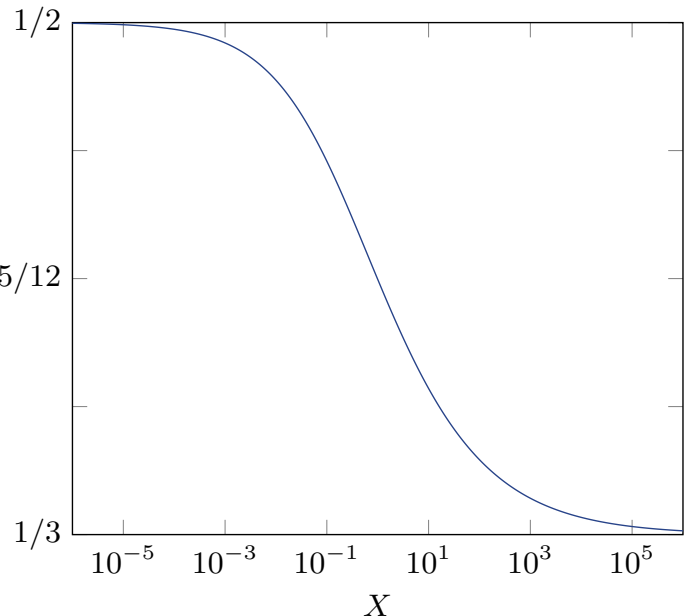

Figure 6.6 - In $(a), g(X)$ is plotted as a function of $X$. In $(b), \beta$ is given as a function of $X$ as obtained from $g(X)$.

The function $g(X)$ is universal, as it does not depend on the slip length $b$. The slip length affects the scaling between $X$ and $\tilde{x} / G z$, so it determines with part of the function $g(X)$ is relevant. When $\tilde{b} \rightarrow 0, X \rightarrow \infty$, while for $\tilde{b} \rightarrow \infty, X \rightarrow 0$.

To evaluate $g(X)$, let $p=s^{3}$. Then

$$
\begin{aligned}
g(X) & =-\frac{1}{2 \pi \mathrm{i}} \int_{c-\mathrm{i} \infty}^{c+\mathrm{i} \infty} \frac{\operatorname{Ai}^{\prime}(s) e^{s^{3} X}}{s^{2} \operatorname{Ai}(s)} 3 s^{2} \mathrm{~d} s \\
& =-\frac{3}{2 \pi \mathrm{i}} \int_{c-\mathrm{i} \infty}^{c+\mathrm{i} \infty} \frac{\operatorname{Ai}^{\prime}(s) e^{s^{3} X}}{\operatorname{Ai}(s)} \mathrm{d} s .
\end{aligned}
$$


The integral should start in the sector $-\pi / 2<\arg (s)<-\pi / 6$ and finish in the sector $\pi / 6<\arg (s)<\pi / 2$. The convergence properties of the integrand are best if we integrate along the rays $\arg (s)= \pm \pi / 3$. On the ray $\infty e^{-\mathrm{i} \pi / 3}$ to the origin we let $s=k e^{-\mathrm{i} \pi / 3}$ with $k$ going from $\infty$ to 0 . On the ray from the origin to $\infty e^{\mathrm{i} \pi / 3}$ we let $s=k e^{\mathrm{i} \pi / 3}$ with $k$ going from 0 to $\infty$. Thus

$$
\begin{aligned}
g(X) & =-\frac{3 e^{-\mathrm{i} \pi / 3}}{2 \pi \mathrm{i}} \int_{\infty}^{0} \frac{\operatorname{Ai}^{\prime}\left(k e^{-\mathrm{i} \pi / 3}\right) e^{-k^{3} X}}{\operatorname{Ai}\left(k e^{-\mathrm{i} \pi / 3}\right)} \mathrm{d} k \\
& -\frac{3 e^{\mathrm{i} \pi / 3}}{2 \pi \mathrm{i}} \int_{0}^{\infty} \frac{\operatorname{Ai}^{\prime}\left(k e^{\mathrm{i} \pi / 3}\right) e^{-k^{3} X}}{\operatorname{Ai}\left(k e^{\mathrm{i} \pi / 3}\right)} \mathrm{d} k,
\end{aligned}
$$

or

$$
\begin{aligned}
g(X) & =\frac{3 e^{-5 \mathrm{i} \pi / 6}}{2 \pi} \int_{0}^{\infty} \frac{\operatorname{Ai}^{\prime}\left(k e^{-\mathrm{i} \pi / 3}\right) e^{-k^{3} X}}{\operatorname{Ai}\left(k e^{-\mathrm{i} \pi / 3}\right)} \mathrm{d} k \\
& +\frac{3 e^{5 \mathrm{i} \pi / 6}}{2 \pi} \int_{0}^{\infty} \frac{\operatorname{Ai}^{\prime}\left(k e^{\mathrm{i} \pi / 3}\right) e^{-k^{3} X}}{\operatorname{Ai}\left(k e^{\mathrm{i} \pi / 3}\right)} \mathrm{d} k .
\end{aligned}
$$

The integrand decays rapidly and can be evaluated numerically. In Fig. 6.6( $a$ ) the function $g(X)$ is plotted as a function of $X$. As demonstrated in the graph, the limiting behaviour of $g(X)$ is as expected: when $X \rightarrow 0, g(X) \propto X^{-1 / 2}$, and when $X \rightarrow \infty, g(X) \propto X^{-1 / 3}$. $\beta$, as obtained by computing the gradient of $\log _{10}(g(X))$ versus $\log _{10}(\tilde{x} / G z)$, is plotted in Fig. 6.6(b). $\beta \approx 5 / 12$ when $X=1$. Thus, the dimensionless group $X$ can be considered as a kind of criterion for the behaviour of $\beta$ : $\beta \rightarrow 1 / 2$ when $X \ll 1$, whereas $\beta \rightarrow 1 / 3$ when $X \gg 1$.

The expression

$$
G(X)=\frac{0.546\left(X^{1 / 2.420}+1.058\right)^{2.420 / 6}}{X^{1 / 2}}
$$

is a good approximation to $g(X)$, which is accurate to about $0.5 \%$ for $10^{-4}<$ $X<10^{4}$. 




\title{
CHAPTER 7
}

\section{Heat and mass transfer over slippery, superhydrophobic surfaces}

\begin{abstract}
The classical Graetz-Nusselt problem is extended to describe heat and mass transfer over heterogeneously slippery, superhydrophobic surfaces. The cylindrical wall consists of segments with a constant temperature/concentration and areas that are insulating/impermeable. Only in the case of mass transport do the locations of hydrodynamic slip and mass exchange coincide. This makes advection near the mass exchanging wall segments larger than near the heat exchanging regions. Also the direction of radial fluid flow is reversed for heat and mass transport, which has an influence on the location where the concentration or temperature boundary layer is compressed or extended. As a result, mass transport is more efficient than heat transfer. Also the influence of axial diffusion on the Nusselt and Sherwood numbers is investigated for various Péclet numbers $P e$. When $P e<10^{2}$, which is characteristic for heat transfer over superhydrophobic surfaces, axial conduction should be taken into account. For $P e \geq 10^{2}$, which are typical numbers for mass transport in microfluidic systems, axial diffusion can be neglected.
\end{abstract}

This chapter has been published as A.S Haase and R.G.H. Lammertink, Heat and mass transfer over slippery, superhydrophobic surfaces, Phys. Fluids 28, 042002 (2016). 


\subsection{Introduction}

The classical Graetz-Nusselt problem considers transport of heat between a flowing medium and a tube, beginning at some location in the hydrodynamically developed flow region. The tube entrance is insulated, and the wall temperature of the heated or cooled section is constant but different from that of the entering fluid temperature. The fluid has constant physical properties, and viscous dissipation and axial heat conduction are neglected. Graetz and Nusselt solved this problem for a uniform velocity profile (no-shear at the wall) [1] and a parabolic velocity profile (no-slip at the wall) $[2,3]$. The amount of local heat transfer is commonly expressed by the local Nusselt number $N u_{x}$, which is a dimensionless heat transfer coefficient. Because of the analogies between heat and mass transfer, the GraetzNusselt solutions are also used to describe mass transfer processes [4]. The mass transfer equivalent of $N u_{x}$ is the local Sherwood number $S h_{x}$.

Previously, we investigated the Graetz-Nusselt problem for homogeneously slippery surfaces [5]. Because homogeneous or intrinsic slip lengths are only of the order of tens of nanometres $[6,7]$, heat/mass transfer can only be enhanced significantly in nanofluidic systems. Intrinsic slip lengths are too small to reduce flow friction substantially in larger systems [8]. Superhydrophobic or microstructured systems, however, can generate effective slip lengths on the order of micrometres, which reduce drag considerably in microscale systems (see, for instance, the review by Rothstein [7]).

The boundary conditions in the Graetz-Nusselt problem, which are no-slip/noshear and a constant temperature/concentration at the wall, are not appropriate in for example membrane systems or superhydrophobic surfaces. Keller and Stein [9] recognised the heterogeneous nature of a membrane and investigated the effects of pore size and spacing on diffusional transport. Juhasz and Deen extended this by considering convective transport $[10,11]$. They described how mass transfer for a non-slippery surface with discrete active mass exchanging areas is affected by the local Péclet number. When the Péclet number increases, convection becomes more important and concentration variations along the surface are minimised. As a result, the surface increasingly resembles a homogeneous surface with a constant wall concentration.

Ling [12] described heat transfer from a single, isothermal strip in a uniform shear flow. Three zones were identified, namely the leading edge, the trailing edge and the zone connecting these edges. For sufficiently large strips or local Péclet numbers, edge effects (axial diffusion) can be neglected and the similarity solution of Lévêque [13] can assumed to be valid everywhere. Ackerberg, Patel and Gupta [14] investigated this problem when heat/mass transfer is dominated by edge effects. This was extended to two-dimensional domains for small Péclet numbers by Phillips [15] and for arbitrary Péclet numbers by Stone [16]. All 
authors found that heat/mass transfer is not uniform over the strip or domain, and that more shear results in increased transfer rates.

When studying transport over heterogeneous surfaces, scientists have often derived and modelled effective boundary conditions that represent a heterogeneous wall [17-19]. Recently, Shah and Shaqfeh [20] studied heat and mass transport in shear flow over a heterogeneous surface containing reactive domains. By using an effective medium theory, they derived expressions for the effective surface reaction rate to replace the details of the heterogeneous surface with a single boundary condition at a certain distance from the non-slippery, reactive wall. When the Péclet number is large, local variations in boundary conditions are screened and heat/mass transport is enhanced.

All examples mentioned so far involve non-slippery surfaces. We previously investigated for a specific type of superhydrophobic surface, which is heterogeneous regarding both momentum and mass transport, how effective wall slip affects mass transport [21]. We showed that convective transport near the surface, as a result of slip, can considerably enhance interfacial mass transfer.

For mass transport over slippery, superhydrophobic surfaces a generalised study such as an extended Graetz-Nusselt problem, is lacking. This is not the case for heat transfer. Maynes, Webb and Davies [22] were the first to investigate (numerically) combined momentum and heat transport in the thermally developed regime over a superhydrophobic surface at constant temperature. They found that thermal transport through a gas-filled cavity to the flowing liquid is at least two orders of magnitude smaller than transport from the solid wall to the liquid. This suggests that the (slippery) gas-liquid interface can be treated as adiabatic. This results in a Nusselt number that is smaller than that for a classical no-slip surface at constant wall temperature. When the Péclet number increases, convective transport becomes more important and the average Nusselt number approaches that for a classical no-slip wall. These observations were confirmed in a semi-analytical work for transverse flow over a superhydrophobic surface containing ribs and cavities at constant heat flux [23]. Later, this work was extended by assessing the influence of axial conduction on thermal transport [24]. The results show that this cannot be neglected for Péclet numbers smaller than approximately $10^{2}$, in particular when the heterogeneity is large compared to the typical channel width.

Enright et al. [25] and Maynes and Crockett [26] studied isoflux thermal transport over superhydrophobic surfaces using effective hydrodynamic and thermal slip lengths. The effective thermal slip length describes an apparent temperature jump between the wall and the liquid, which accounts for the adiabatic part of the heterogeneous surface. Assuming that heat transport near the surface is purely diffusive, Enright et al. derived an expression for the thermal slip length, which was confirmed by simulations. Apparent hydrodynamic and thermal slip lengths 
behave similarly and have comparable values [25-27]. Steigerwalt Lam et al. [28] used hydrodynamic and thermal slip lengths to study thermally developing Couette flow. Moreira and Bandaru [29] used an effective medium approach to study the thermal conductivity of superhydrophobic surfaces.

It appears little research has been done on convective heat/mass transport over heterogeneous and slippery surfaces, as recognised by others [20, 22]. The Graetz-Nusselt problem for superhydrophobic surfaces has only been studied for thermal transport. Mass transfer has not been considered, while in that case the no-slip segment instead of the no-shear area is impermeable, and the no-shear region instead of the non-slippery part of the wall is exchanging mass with the fluid flow [21]. Often unidirectional flow is assumed, thereby neglecting transverse velocity components present near heterogeneously slippery surfaces. Besides that, most research has been directed towards thermally developed transport.

This study aims to be a natural extension of our previous work [5] by changing the boundary conditions from uniform to heterogeneous, for both momentum and heat/mass transport. As far as we know, it is the first study to discuss the Graetz-Nusselt problem for mass transfer over superhydrophobic surfaces and to compare this with thermal transport over such surfaces. We also investigate the influence of axial conduction on heat/mass transport for these systems.

\subsection{Methodology}

\subsubsection{Governing equations}

In Fig. 7.1 a schematic representation is given of the system considered in this study. The flow in the tube is hydrodynamically fully developed and has a uniform temperature $T_{0}$ when it enters the heated or cooled section of the tube. The wall temperature $T_{1}$ of the no-slip wall segments is constant, but different from that of the entering fluid temperature $T_{0}$. The fluid has constant physical properties, and viscous dissipation is neglected. Analogously, when considering mass transport, the inlet concentration is $c_{0}$, and the wall concentration $c_{1} \neq c_{0}$ of the no-shear wall segments is constant.

We assume that the Reynolds number $R e=u_{a v} D / \nu$ is small, where $u_{a v}$ is the average liquid velocity, $D$ is the tube diameter and $\nu$ is the kinematic viscosity. In that case momentum transport transport is described by the continuity and Stokes equations,

$$
\begin{gathered}
\frac{1}{r} \frac{\partial}{\partial r}\left(r u_{r}\right)+\frac{\partial u_{x}}{\partial x}=0 \\
0=-\frac{\partial p}{\partial r}+\mu\left[\frac{\partial}{\partial r}\left(\frac{1}{r} \frac{\partial}{\partial r}\left(r u_{r}\right)\right)+\frac{\partial^{2} u_{r}}{\partial x^{2}}\right]
\end{gathered}
$$




$$
0=-\frac{\partial p}{\partial x}+\mu\left[\frac{1}{r} \frac{\partial}{\partial r}\left(r \frac{\partial u_{x}}{\partial r}\right)+\frac{\partial^{2} u_{x}}{\partial x^{2}}\right],
$$

where $p$ is the pressure, $\mu$ is the dynamic viscosity and $u_{i}$ is the velocity in $i$-direction.

Stationary heat transport in an axisymmetric cylindrical system, under the assumption of constant density $\rho$, heat capacity $C_{p}$ and thermal conductivity $k$, is described as

$$
u_{r} \frac{\partial T}{\partial r}+u_{x} \frac{\partial T}{\partial x}=\alpha\left[\frac{1}{r} \frac{\partial}{\partial r}\left(r \frac{\partial T}{\partial r}\right)+\frac{\partial^{2} T}{\partial x^{2}}\right],
$$

where $\alpha=k /\left(\rho C_{p}\right)$ is the thermal diffusivity. Axial conduction $\left(\partial_{x}^{2} T\right)$ can be neglected when it is much smaller than axial convection. When replacing $T$ for the concentration $c$, and $\alpha$ for the diffusion coefficient $\mathcal{D}$, which is also constant, this equation describes stationary mass transport.

The continuity and Stokes equations are made dimensionless using $\tilde{x}=x / R$, $\tilde{r}=r / R, \tilde{u}_{i}=u_{i} / u_{a v}$ and $\tilde{p}=p R / \mu u_{a v}$, which then read

$$
\begin{gathered}
\frac{1}{\tilde{r}} \frac{\partial}{\partial \tilde{r}}\left(\tilde{r} \tilde{u}_{r}\right)+\frac{\partial \tilde{u}_{x}}{\partial \tilde{x}}=0 \\
0=-\frac{\partial \tilde{p}}{\partial \tilde{r}}+\frac{\partial}{\partial \tilde{r}}\left(\frac{1}{\tilde{r}} \frac{\partial}{\partial \tilde{r}}\left(\tilde{r} \tilde{u}_{r}\right)\right)+\frac{\partial^{2} \tilde{u}_{r}}{\partial \tilde{x}^{2}}, \\
0=-\frac{\partial \tilde{p}}{\partial \tilde{x}}+\frac{1}{\tilde{r}} \frac{\partial}{\partial \tilde{r}}\left(\tilde{r} \frac{\partial \tilde{u}_{x}}{\partial \tilde{r}}\right)+\frac{\partial^{2} \tilde{u}_{x}}{\partial \tilde{x}^{2}} .
\end{gathered}
$$

The convection-diffusion equation is non-dimensionalised using $\Theta=\left(T_{1}-\right.$ $T) /\left(T_{1}-T_{0}\right)$, giving

$$
\tilde{u}_{r} \frac{\partial \Theta}{\partial \tilde{r}}+\tilde{u}_{x} \frac{\partial \Theta}{\partial \tilde{x}}=\frac{2}{P e}\left[\frac{1}{\tilde{r}} \frac{\partial}{\partial \tilde{r}}\left(\tilde{r} \frac{\partial \Theta}{\partial \tilde{r}}\right)+\frac{\partial^{2} \Theta}{\partial \tilde{x}^{2}}\right] .
$$

The Péclet number, which gives the ratio of advective to diffusive transport, is defined as

$$
P e=\operatorname{Re} P r=\frac{u_{a v} D}{\alpha},
$$

where $\operatorname{Pr}=\nu / \alpha$ is the Prandtl number. The domain in $\tilde{x}$-direction runs from zero to any finite value.

The rate of heat transport can be described with a dimensionless correlation of the form $N u_{x}=f_{1}(R e, \operatorname{Pr}, x / L)$. Based on the analogies between heat and mass transport [4], the mass transfer rate for the same geometry and for analogous boundary conditions can be described by $S h_{x}=f_{2}(R e, S c, x / L)$. Thus, replacing 
$\Theta$ in Eq. (7.6) for the dimensionless concentration $\Theta_{m}=\left(c_{1}-c\right) /\left(c_{1}-c_{0}\right)$ yields the dimensionless convection-diffusion equation for mass transport. In that case, $P e=R e S c$, with $S c=\nu / \mathcal{D}$ being the Schmidt number.

\subsubsection{Nusselt/Sherwood number}

The local Nusselt number $N u_{x}$ is defined as

$$
N u_{x}=\frac{h_{x} D}{k},
$$

where $h_{x}$ is the local heat transfer coefficient. Using Fourier's law of thermal conduction, the local heat transfer coefficient $h_{x}$ can be written as $h_{x}=$ $-k /\left.\left(\langle T\rangle-T_{1}\right) \partial_{r} T\right|_{r=R}$. The temperature gradient can be rewritten in dimensionless form using the flow-averaged temperature $\langle\Theta\rangle=\left(T_{1}-\langle T\rangle\right) /\left(T_{1}-T_{0}\right)$. This yields

$$
N u_{x}=-\left.\frac{2}{\langle\Theta\rangle} \frac{\partial \Theta}{\partial \tilde{r}}\right|_{\tilde{r}=1} .
$$

The equivalent of the Nusselt number for mass transport is the Sherwood number:

$$
S h_{x}=\frac{m_{x} D}{\mathcal{D}} .
$$

Here, $m_{x}=-\mathcal{D} /\left.\left(\langle c\rangle-c_{1}\right) \partial_{r} c\right|_{r=R}$ is the local mass transfer coefficient. Using the flow-averaged concentration $\left\langle\Theta_{m}\right\rangle=\left(c_{1}-\langle c\rangle\right) /\left(c_{1}-c_{0}\right)$ we find for the local Sherwood number

$$
S h_{x}=-\left.\frac{2}{\left\langle\Theta_{m}\right\rangle} \frac{\partial \Theta_{m}}{\partial \tilde{r}}\right|_{\tilde{r}=1} .
$$

If $\tilde{x} \rightarrow \infty, N u_{x} \rightarrow N u_{\infty}$ and $S h_{x} \rightarrow S h_{\infty}$ when averaged over the heterogeneities at the wall.

\subsubsection{Boundary conditions}

Various cases are studied here, all being characterised by a different set of boundary conditions (see Fig. 7.1). The boundary conditions for momentum transport are always the same, though. These are rotational symmetry in the middle of the tube,

$$
\frac{\partial \tilde{u}_{r}}{\partial \tilde{r}}=0 \text { at } \tilde{r}=0
$$

and a regular pattern of no-slip and no-shear (a surface free from stress) at the wall (see Fig. 7.1 for the $\tilde{x}$-positions),

$$
\begin{cases}\tilde{\mathbf{u}}=0 & \text { at } \tilde{r}=1 \text { and } \tilde{x}=\{\text { no-slip areas }\} \\ \tilde{\mathbf{u}} \cdot \mathbf{n}=0 \text { and } & \text { at } \tilde{r}=1 \text { and } \tilde{x}=\{\text { no-shear areas }\} \\ \left(-\tilde{p} \mathbf{I}+\left(\nabla \tilde{\mathbf{u}}+(\nabla \tilde{\mathbf{u}})^{\mathrm{T}}\right)\right) \tilde{\mathbf{n}}=0 & \end{cases}
$$


In all cases the inlet temperature or concentration is constant, being

$$
\Theta(\tilde{r}, 0)=1
$$

Case $C$. In the first case, the wall temperature is constant $(\mathrm{C})$, as could be the case in for instance surfaces containing patches that are infused with a lubricating liquid [30]. This gives the following boundary condition at $\tilde{r}=1$ :

$$
\Theta(1, \tilde{x})=0
$$

Case T. This case describes the extended Graetz-Nusselt problem for thermal (T) transport. The slippery patches correspond to gas present in the micro-structures of the slippery, superhydrophobic surface. Because the thermal conductivity of a gas is much smaller than that of a solid, the slippery (gas) patches can be considered as insulating [22]. Therefore,

$$
\begin{cases}\Theta=0 & \text { at } \tilde{r}=1 \text { and } \tilde{x}=\{\text { no-slip areas }\} \\ \partial \Theta / \partial r=0 & \text { at } \tilde{r}=1 \text { and } \tilde{x}=\{\text { no-shear areas }\}\end{cases}
$$

Case $M$. The last case describes the extended Graetz-Nusselt problem for mass (M) transport. This means that the boundary conditions are reversed compared to case $T$. The heterogeneous wall could be considered as a membrane, used to contact a gas and liquid with each other [21]. In that case, mass transfer takes only place between the liquid and the gas. The non-slippery solid wall can be considered as impermeable. Then

$$
\begin{cases}\partial \Theta_{m} / \partial r=0 & \text { at } \tilde{r}=1 \text { and } \tilde{x}=\{\text { no-slip areas }\} \\ \Theta_{m}=0 & \text { at } \tilde{r}=1 \text { and } \tilde{x}=\{\text { no-shear areas }\}\end{cases}
$$

Regarding the positioning of the no-shear and adiabatic/impermeable wall segments, one can maintain either the same position of the zero-flux regions among all three cases, or keep the location of the no-shear regions the same. These two options give the same result when the number of gas/solid modules becomes very large. Here it is decided to choose for the first option, implying that the positions of the no-shear and no-slip segments are reversed when switching from heat to mass transport.

\subsubsection{Effective slip length}

Wall slip is usually described using Navier's slip condition [7, 31],

$$
u_{b}=-\left.b \frac{\partial u}{\partial r}\right|_{r=R}
$$


Flow profile (case $M)$

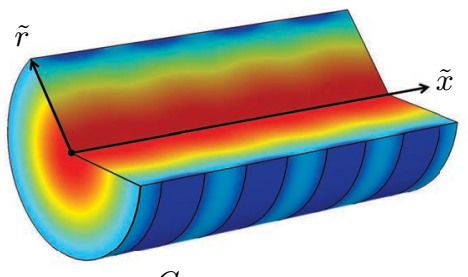

case $C$
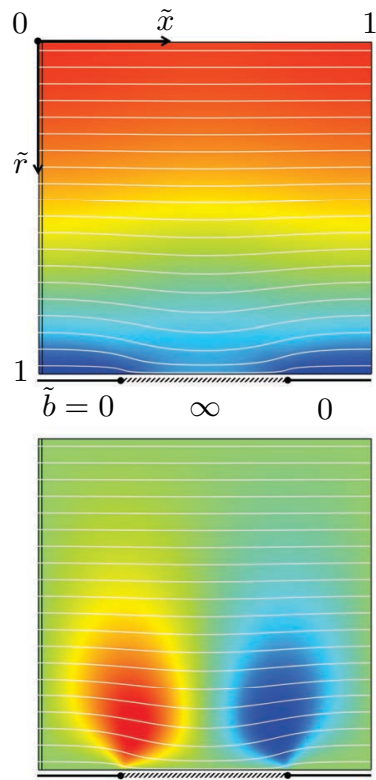

$\tilde{b}=0$

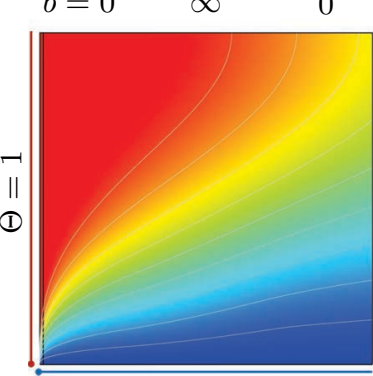

$\Theta=0$

1
Concentration profile $(P e=10$, case $M)$

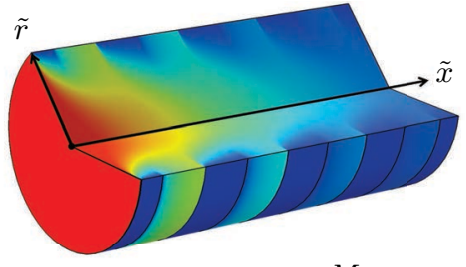

case $M$
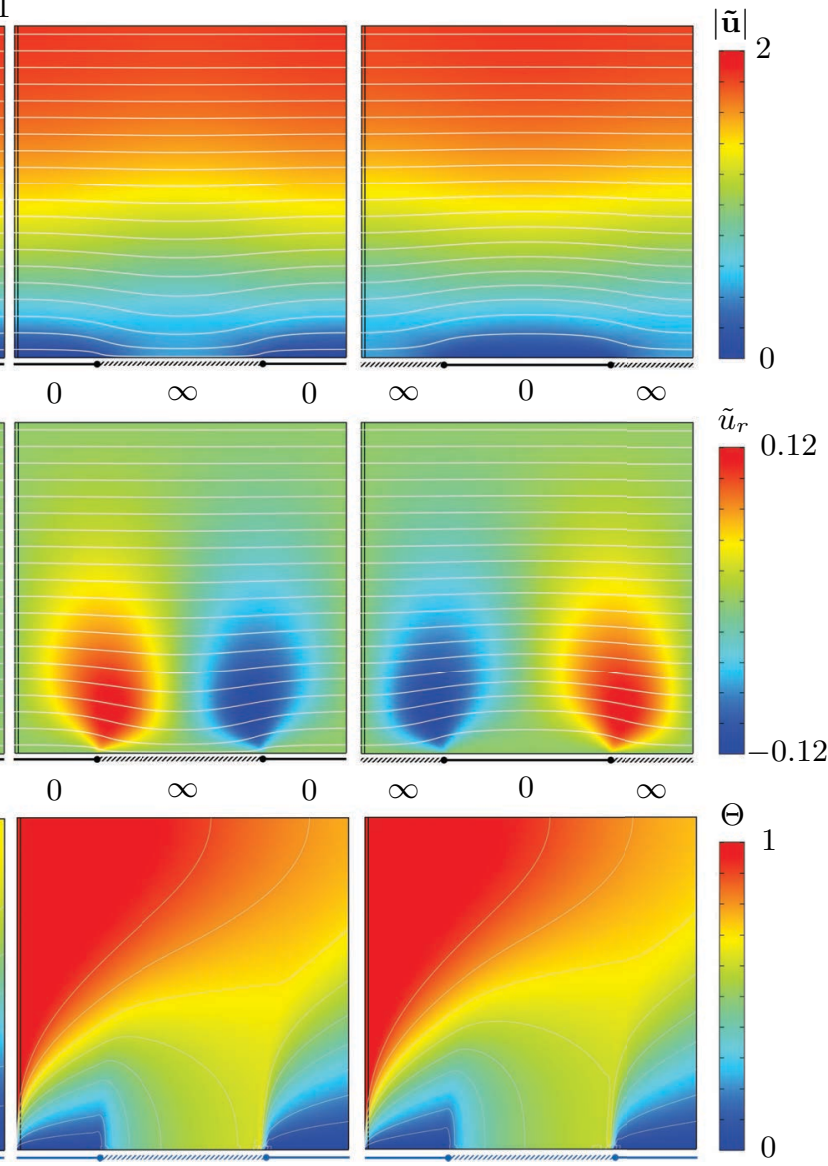

$\Theta=0 \quad \partial_{r} \Theta=0 \quad \Theta=0 \quad \Theta=0 \quad \partial_{r} \Theta=0 \quad \Theta=0$

Figure 7.1 - Illustration of the various physical systems studied. For the figures shown here, $L=R$ and $P e=10$. The streamlines in the plots of the velocity magnitude $|\tilde{\mathbf{u}}|$ and the radial velocity component $\tilde{u}_{r}$ have a fixed starting position. The contour lines in the dimensionless temperature/concentration plots are given for $\Theta=0.1 p$ with $p=\{1,2, \ldots, 9\}$. 
where $u_{b}$ is the slip velocity at the wall, and $b$ is the slip length. In the case of a homogeneously slippery wall, this slip length has a uniform value, whereas for a heterogeneously slippery wall like a superhydrophobic surface, the local value of $b$ varies. The slip length required to obtain a homogeneous slip flow with the same average velocity $u_{a v}$ and pressure gradient $\partial p / \partial x$ as a flow over a heterogeneously slippery surface is referred to as the effective slip length $b$. For Stokes flow we find for the average velocity $u_{a v}$ of a homogeneous slip flow through a cylinder that

$$
u_{a v}=-\frac{\partial p}{\partial x}\left(\frac{R^{2}}{8 \mu}+\frac{b R}{2 \mu}\right) .
$$

Rewriting this equation and making it dimensionless using $\tilde{b}=b / R$, we obtain for a flow with $\tilde{u}_{a v}=1$ the following expression for the (effective) slip length $\tilde{b}$ :

$$
\tilde{b}=-\frac{1}{4}\left(\frac{8}{\partial \tilde{p} / \partial \tilde{x}}+1\right) \text {. }
$$

The analytical solution derived by Davis and Lauga [32] for a heterogeneously slippery surface can be used to estimate the (maximum) amount of slip. As derived before [21], for flat bubbles

$$
\frac{2 b}{\varepsilon L_{g}} \approx \frac{\pi}{8}
$$

In order to rewrite this, we derive that $L_{g}=\varepsilon R / n$, since the length of a no-slip $\left(L_{s}\right)$ and a no-shear $\left(L_{g}\right)$ wall segment is $L_{s}+L_{g}=R / n$. Here, $n$ is the number of no-slip/no-shear units in a tube of length $R$. Substitution gives

$$
\tilde{b} \approx \frac{\pi}{16} \frac{\varepsilon^{2}}{n}
$$

This means that for our system, with $\varepsilon=1 / 2$ and $n=1$, the amount of slip is $\tilde{b}=\pi / 64 \approx 0.05$. This corresponds closely to what is obtained from the simulations $(\tilde{b}=0.0542)$.

\subsection{Numerical approach}

COMSOL Multiphysics 5.0 was used to compute the velocity and temperature/concentration profiles. For solving the flow profile, the laminar flow module was used. Heat/mass transport was modelled utilising the convection-diffusion equation. Standard relative tolerance was $1 \times 10^{-3}$. P2 $+\mathrm{P} 1$ discretisation (second order elements for velocity and first order elements for pressure) was used to solve the Stokes equations. Quadratic elements were used for solving the convection- 
diffusion equation.

From $\tilde{x}=\left[0,10^{-2}\right]$ a logarithmically spaced mesh was used. The length of this mesh was varied. For very short domains, the logarithmic mesh ran from $\tilde{x}=\left[10^{-7}, 10^{-2}\right]$, whereas for long domains this was $\tilde{x}=\left[10^{-3}, 10^{-2}\right]$. The mesh in the $\tilde{r}$-direction was defined as $\tilde{r}=1-\left[0.99\left(1-\tilde{r}_{0}^{2}\right)+0.01\left(1-\tilde{r}_{0}\right)\right]$, with $\tilde{r}_{0}$ being a linearly spaced vector with 101 grid points. This mesh was also defined at $\tilde{x}=1$. For $\tilde{x}=\left(10^{-2}, L / R\right]$ a free triangular mesh was used, the mesh being refined near the boundaries with 8 boundary layers. The standard mesh size was set to 'extra fine', resulting in a total number of approximately $29 \times 10^{3}$ domain elements and 670 boundary elements for a domain of length $L / R=1$. When solving the model for very small no-shear areas, the meshing was further refined by setting the mesh size to 'extremely fine'. This gave about $80 \times 10^{3}$ domain elements and 840 boundary elements for a domain of size $1 \times 1$.

Since the fluid flow is fully periodic and does not depend on the temperature/concentration field, the flow field was solved first for $\tilde{u}_{a v}=1$. Subsequently, using the obtained velocity profile, the temperature/concentration profiles were computed. To obtain developed Nusselt/Sherwood numbers, the outlet temperature/concentration profile of domain $j$ was used as the inlet profile of domain $j+1$. This procedure was repeated until the Nusselt/Sherwood numbers in the domain did not change anymore. Subsequently the developed Nusselt $N u_{\infty}$ and Sherwood $S h_{\infty}$ numbers were computed.

\subsection{Results and discussion}

\subsubsection{Local Nusselt/Sherwood number}

In Fig. 7.1 the three cases considered in this study are displayed. The Péclet number is the same for all cases, which is $P e=10^{1}$. We observe that the velocity profiles are different for the three cases. At the start of the heat/mass exchanging wall segments, radial advection is directed inwards for case $T$, but is directed outwards for case $M$. As will be discussed later, this affects the transfer rate of heat/mass, depending on the Péclet number. The rate of heat/mass transfer at each position $\tilde{x}$ is expressed by the local Nusselt $N u_{x}$ or Sherwood number $S h_{x}$. These numbers, which are proportional to the gradient at the wall and the inverse of the flow-averaged temperature/concentration, are obtained from the temperature/concentration profiles. Although these profiles for case $T$ and case $M$ look very similar, there are some differences. These differences can be related to the different boundary conditions and become more pronounced for increasing Péclet number $P e$.

In Fig. 7.2(a) the local Nusselt $N u_{x}$ and Sherwood $S h_{x}$ numbers are plotted. First, the fluid flow normal to the surface leads to large variations in the curve 
for constant wall temperature. The profile for case $C$ even crosses the classical Graetz-Nusselt profile for no-slip flow (solid line). We also observe that locally $N u_{x}$ or $S h_{x}$ is much larger than the transport number for a homogeneous (slip) flow with a constant wall temperature/concentration (dashed line). These variations have been observed before [22, 26], although not when the flow is described as a uni-directional slip flow $[23-26,28]$. Although case $C$ does not represent a superhydrophobic surface, the set of boundary conditions does describe slippery, liquid-infused surfaces with patterned wettability [30] when the surface and the infusing liquid have comparable thermal conductivity.

Second, the slope of the profiles in the developing regime, i.e. for $\tilde{x}<10^{-1}$ where the flow-averaged temperature/concentration is essentially unity $(\langle\Theta\rangle=1)$, is different for heat and mass transfer. Whereas the exponent of the slope is $-1 / 3$ for heat transport (no-slip wall adjacent to entrance), it is $-1 / 2$ for mass transport (no-shear wall adjacent to entrance). This is in agreement with the analytical values for the exponent $\beta$ found by Lévêque, who predicted that $N u_{x}, S h_{x} \propto \tilde{x}^{-\beta}$ in the developing regime $[5,13]$.

Finally, Fig. 7.2(a) reveals that for case $T$, heat transport is enhanced when the flow is about to enter a no-shear wall segment where $\partial_{r} \Theta=0$. For case $M$ the situation is exactly opposite, as mass transport is retarded when the flow enters a no-slip region where $\partial_{r} \Theta_{m}=0$.

To investigate the influence of the different set of boundary conditions on the rate of heat/mass transport in more detail, we plotted the flow-averaged temperature $\langle\Theta\rangle$ and concentration $\left\langle\Theta_{m}\right\rangle$ in Fig. 7.2(b), and the temperature/concentration gradient $-\partial_{r} \Theta$ at $\tilde{r}=1$ in Fig. 7.2(c). The profiles in Fig. 7.2(b) show that above the adiabatic/impermeable wall segments the amount of energy/mass in the flow does not change, as no heat/mass is exchanged with the wall. The distribution of heat/mass above these segments does change, however, as the plots in Fig. 7.1 reveal. We also observe that the set of boundary conditions for case $C$ leads to the most efficient cooling of the liquid flow. Between case $T$ and case $M$ the differences are smaller, although the flow-averaged concentration decreases faster than the temperature. This already indicates that the differences in boundary conditions for case $T$ and case $M$ make mass transport more efficient than heat transfer between wall and fluid. On the other hand, however, Fig. 7.2(c) reveals that the difference between the gradients for case $T$ and case $M$ becomes larger with position $\tilde{x}$, the gradient for case $M$ being smaller than for case $T$. Since $N u_{x},\left.S h_{x} \propto \partial_{r} \Theta\right|_{\tilde{r}=1} /\langle\Theta\rangle$, this compensates for the increasing difference in flow-averaged temperature/concentration. For that reason, as Fig. 7.2 $(d)$ shows, the ratio of Nusselt to Sherwood number is repetitive in the developed regime $\left(\tilde{x}>1\right.$ for $\left.P e=10^{1}\right)$.

The precise value of the Nusselt/Sherwood number above the heat/mass exchanging wall segments strongly varies. This has various physical origins. First, 
(a) Nusselt/Sherwood numbers

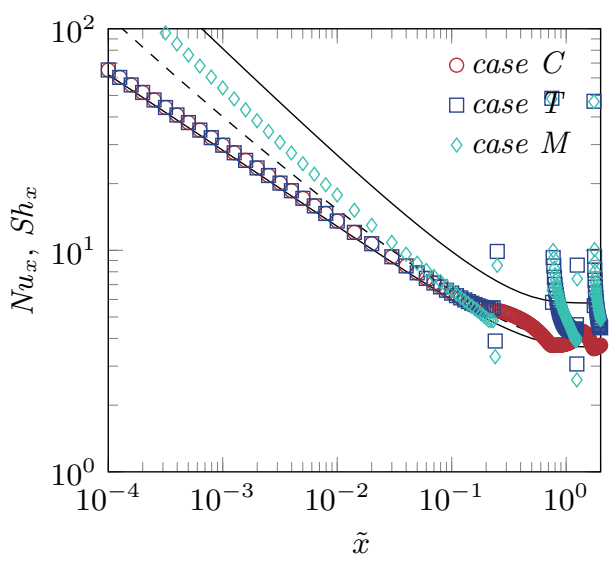

(c) Temperature/concentration gradients

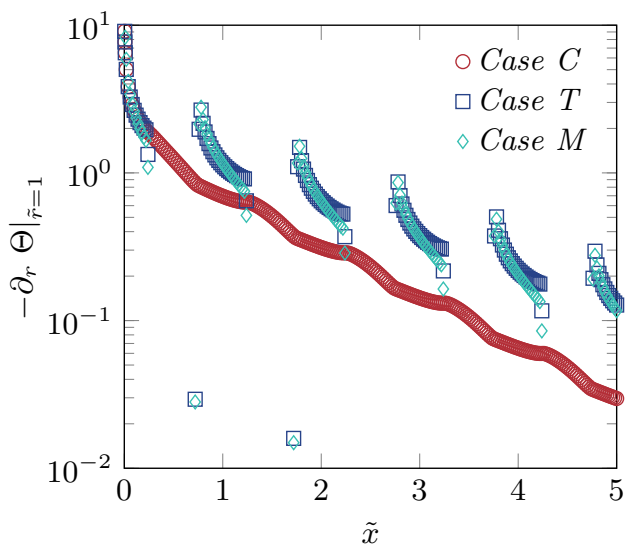

(b) Flow-averaged temperature/concentration

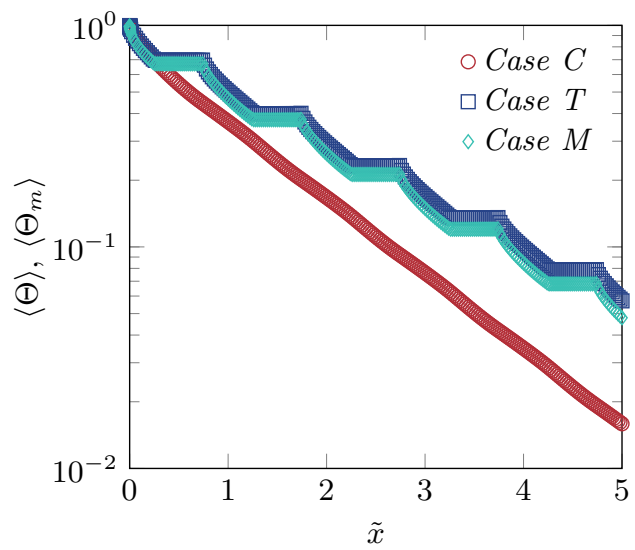

(d) Comparison of case $T$ with case $M$

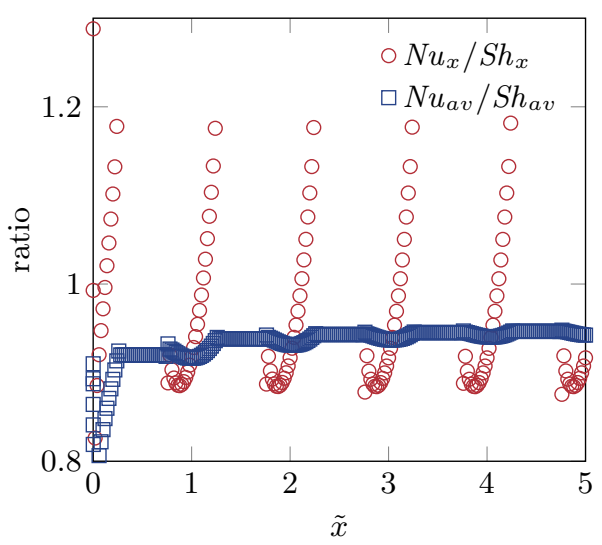

Figure 7.2 - For all graphs, $P e=10^{1}, \tilde{b}=0.0542$ and there is no axial diffusion. In (a), the local Nusselt $N u_{x}$ and Sherwood numbers $S h_{x}$ are plotted for all three cases on a logarithmic scale. The solid lines are the classical Graetz-Nusselt solutions for no-slip and no-shear flow. The dashed curve is the profile for a homogeneous slip flow with a constant wall temperature/concentration and having the same slip length $\tilde{b}$ as the superhydrophobic surface. Both Nusselt and Sherwood number are proportional to the temperature/concentration gradient and the inverse of the flow-averaged temperature/concentration, i.e. $N u_{x},\left.S h_{x} \propto \partial_{r} \Theta\right|_{\tilde{r}=1} /\langle\Theta\rangle$. The flow-averaged temperatures $\langle\Theta\rangle$ and concentration $\left\langle\Theta_{m}\right\rangle$ are shown in $(b)$, while the temperature/concentration gradients $\partial_{r} \Theta$ at $\tilde{r}=1$ are given in $(c)$. In $(d)$ the ratios of both local and average Nusselt (case T) to Sherwood number (case $M$ ) are plotted. For the ratio $N u_{x} / S h_{x}$ the data points above the adiabatic/impermeable wall segments are omitted, as division of very small numbers $\left(N u_{x}, S h_{x} \rightarrow 0\right)$ gives spurious results. 
we observe that both flow-averaged temperature/concentration (Fig. 7.2(b)) and the gradients (Fig. 7.2(c)) for case $T$ and case $M$ are high at the beginning of a heat/mass exchanging wall segment, but drop very quickly, contrary to the profiles for case $C$. Inspection of Fig. 7.1 shows that above the adiabatic/impermeable regions homogenisation of heat/mass takes place, which leads to high gradients at the beginning of each heat/mass exchanging wall segment. This suggests that, apart from the entrance of the tube, transport above all these segments is characterised by a developing regime. Second, the heat exchanging area is non-slippery, while the mass exchanging wall segment is slippery. Wall slip promotes transport, in particular in the developing regime, as classical theories [1-3, 5, 13] show and as in agreement with the profiles in Fig. 7.2( $a)$. Convection near these segments is therefore larger for case $M$. This is also supported by Fig. $7.2(d)$, showing that above the heat/mass exchanging wall segments initially $N u_{x} / S h_{x}<1$ (mass transport is faster). Third, as Fig. 7.1 reveals, the direction of radial advection at the beginning/end of each heat/mass exchanging wall segment is reversed for case $T$ and case $M$. At the beginning of these segments, the concentration/temperature boundary layer is compressed for case $M$ (leading to larger gradients), but extended for case $T$ (resulting in smaller gradients). This may explain why at the beginning of these segments the ratio $N u_{x} / S h_{x}$ drops, then reaches a minimum, and at the end increases to values of $N u_{x} / S h_{x}>1$ (thermal transport is faster).

These three phenomena make the ratio of heat to mass transfer non-symmetric around the midpoints of the heat/mass exchanging wall segments, as Fig. 7.2 $(d)$ shows. Consider for instance the second segment located at $0.75<\tilde{x}<1.25$. For $0.75<\tilde{x}<1.1$ mass transport is faster $\left(N u_{x} / S h_{x}<1\right)$, while for $1.1<\tilde{x}<1.25$ heat transfer is faster $\left(N u_{x} / S h_{x}>1\right)$. As a result, the average Sherwood number is always larger than the average Nusselt number, i.e. $N u_{a v} / S h_{a v}<1$ (Fig. $7.2(d)$ ), and the flow-averaged concentration is smaller than the mixing-cup temperature, i.e. $\left\langle\Theta_{m}\right\rangle\langle\langle\Theta\rangle$ (Fig. 7.2(b)). We therefore establish that, considering case $T$ and case $M$, on average mass transport is faster than thermal transport.

Fig. 7.3 shows that with increasing Péclet number, the variations in the local transport coefficient, in this case the local Nusselt $N u_{x}$ (case $T$ ) and Sherwood number $S h_{x}($ case $M)$, become more pronounced. Since axial diffusion is not taken into account here, an increasing Péclet number implies that the ratio of (radial) advection to radial diffusion increases. Because of the different flow profiles for case $T$ and case $M$, this clarifies why the differences between $N u_{x}$ and $S h_{x}$ also increase with increasing $P e$. However, as indicated in Table 7.1, it should be noticed that for heat transport over superhydrophobic surfaces in a microfluidic platform typically $P e<10^{2}$, whereas for mass transfer generally $P e \geq 10^{2}$. 
(a) Case $T$, no axial diffusion, $\tilde{b}=0.0542$

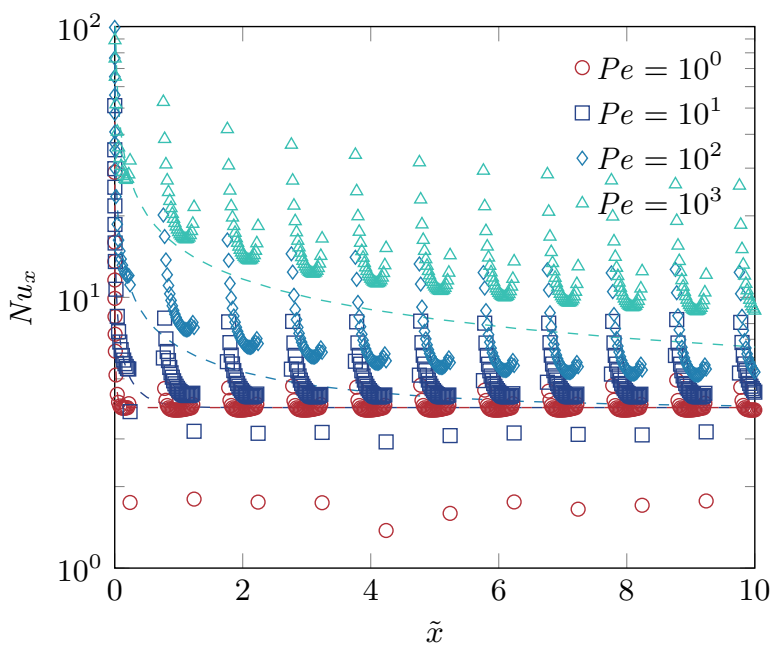

(b) Case $M$, no axial diffusion, $\tilde{b}=0.0542$

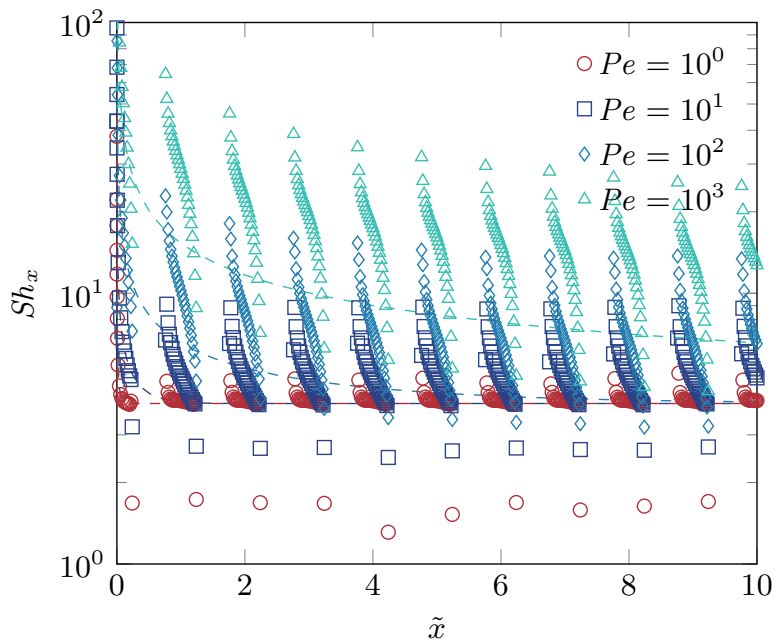

Figure 7.3 - These figures show the influence of the Péclet number $P e$ on heat/mass transport. In (a) the local Nusselt number $N u_{x}$ and in $(b)$ the local Sherwood number $S h_{x}$ is plotted versus the dimensionless position $\tilde{x}$. For both figures, the length is $L=10 R$ and the number of no-slip/no-shear units per length $R$ is $n=1$. The dashed curves are the Nusselt/Sherwood profiles for a homogeneous slip flow with a constant wall temperature/concentration and having the same slip length $\tilde{b}$ as the superhydrophobic surface. 
Table 7.1 - Typical values of relevant dimensionless numbers for a microfluidic system.

\begin{tabular}{lcccccc}
\hline & $D[\mathrm{~m}]$ & $L[\mathrm{~m}]$ & $R e$ & $P r$ & $S c$ & $P e$ \\
\hline Thermal transport & $10^{-4}$ & $10^{-2}$ & $10^{-1}$ & $10^{1}$ & - & $10^{0}$ \\
Mass transport & $10^{-4}$ & $10^{-2}$ & $10^{-1}$ & - & $10^{3}$ & $10^{2}$ \\
\hline
\end{tabular}

\subsubsection{Average Nusselt/Sherwood number}

For a heterogeneous surface as considered here, the local transport number can be much higher than for a homogeneously slippery surface with a constant wall temperature/concentration [5, 33, 34]. However, the adiabatic/impermeable wall segments have an adverse effect on the average Nusselt $N u_{a v}$ or Sherwood $S h_{a v}$ number, which are plotted in Fig. 7.4(a) and (b) for case $T$ and case $M$, respectively. For that reason, fluctuations in the average Nusselt/Sherwood numbers are not so large as in the local transport numbers. The fluctuations become smaller with increasing position $\tilde{x}$, because local variations are averaged out.

Fig. 7.4( $a$ ) shows that the difference between the average Nusselt number $N u_{a v}$ and the corresponding classical Graetz-Nusselt solution becomes smaller with increasing Péclet number $P e$. This is supported by the data for case $T$ (no axial diffusion) in Fig. 7.5, where the developed Nusselt number $N u_{\infty}$ is plotted as function of the Péclet number. $N u_{\infty}$ may even be slightly larger than the classical value of 3.66. This requires very large $P e$, though. It can be attributed to slip near the wall, because for non-slippery surfaces the Nusselt profile would approach the classical Graetz-Nusselt solution [10, 11]. The reason why transport increases with Péclet number is that advection in both axial and radial direction increasingly dominates over diffusion. For large $P e$, the no-shear, adiabatic regions are 'less effective at interrupting the thermal and hydrodynamic boundary layers' [22]. Heat is 'being swept downstream' so fast, that the effect of the 'tangential flow is to smooth out concentration variations' [10].

The behaviour of the average Sherwood number $S h_{a v}$ is analogous to that of the average Nusselt number $N u_{a v}$, as Fig. 7.4(b) shows. Comparison of the average Nusselt and Sherwood numbers reveals that $S h_{a v}>N u_{a v}$, and that the difference between heat and mass transfer becomes larger with increasing Péclet number. While $N u_{a v}$ approaches the classical Graetz-Nusselt profile for no-slip flow, $S h_{a v}$ exceeds this profile and starts to overlap with the solution for homogeneous slip flow with constant wall concentration [5]. This observation is supported by Fig. 7.5, showing that the developed Sherwood number is larger than the developed Nusselt number (no axial diffusion), i.e. $S h_{\infty}>N u_{\infty}$. This clearly illustrates that the difference in boundary conditions for heat/mass transfer affects the rate of transport. A coinciding location of hydrodynamic slip and mass transfer is 
(a) Case $T$, no axial diffusion, $\tilde{b}=0.0542$

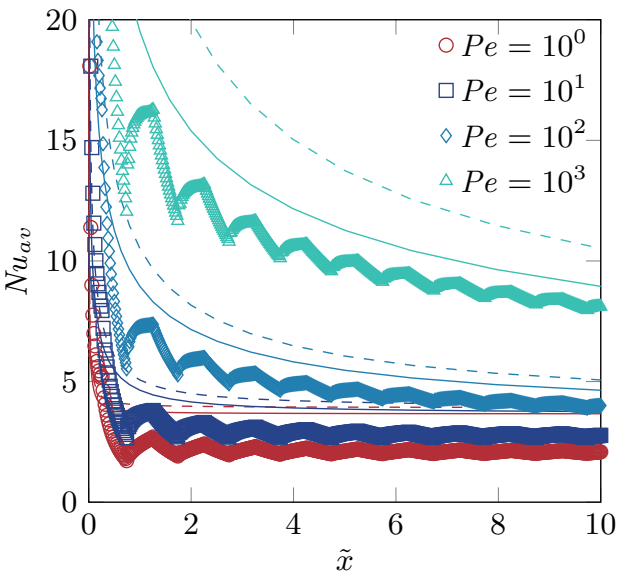

(c) Case $T$, axial diffusion, $\tilde{b}=0.0542$

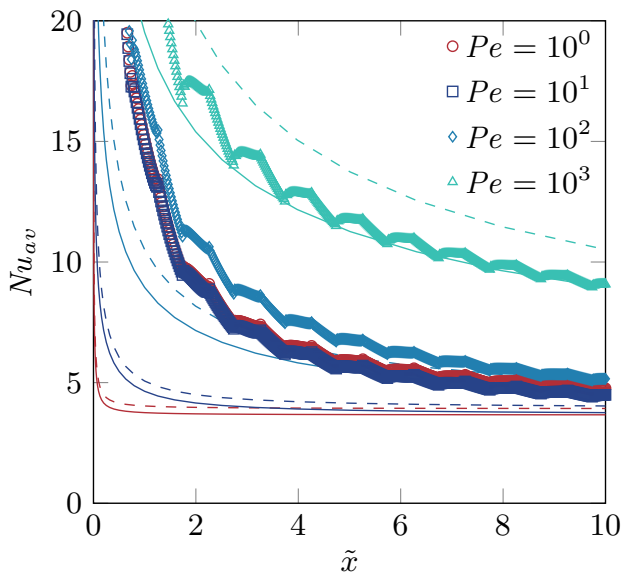

(b) Case $M$, no axial diffusion, $\tilde{b}=0.0542$

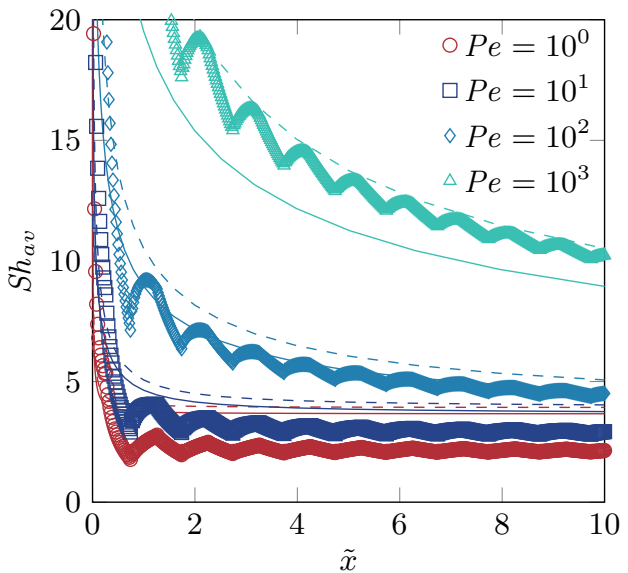

(d) Case $M$, axial diffusion, $\tilde{b}=0.0542$

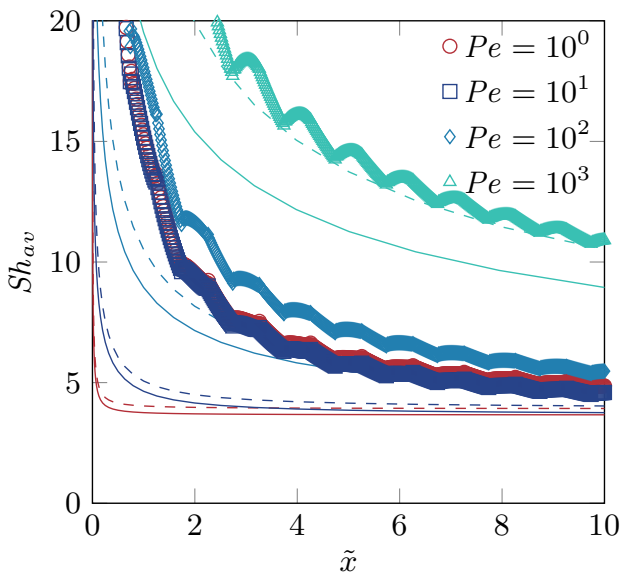

Figure 7.4 - Local average Nusselt $N u_{a v}$ and Sherwood $S h_{a v}$ number for case $T$ and case $M$ for $n=1$ and $L=10 R$ as a function of the Péclet number $P e$. In $(a)$ and $(b)$ axial diffusion is neglected, whereas $(c)$ and $(d)$ include the effect of axial diffusion. The solid lines are the classical Graetz-Nusselt solutions for Hagen-Poiseuille flow. The dashed curves are the Nusselt/Sherwood profiles for a homogeneous slip flow with a constant wall temperature/concentration and having the same slip length $\tilde{b}$ as the superhydrophobic surface.

beneficial for the overall transport rate, as was discussed in Sec. 7.4.1.

The relative magnitude of radial convection to radial diffusion, for a given Péclet number, can be altered when changing the number of no-slip/no-shear units $n$ per length $R$. The influence of this number $n$ on mass transport is shown in Fig. 7.6. When $n$ becomes larger, local fluctuations in the Sherwood number 


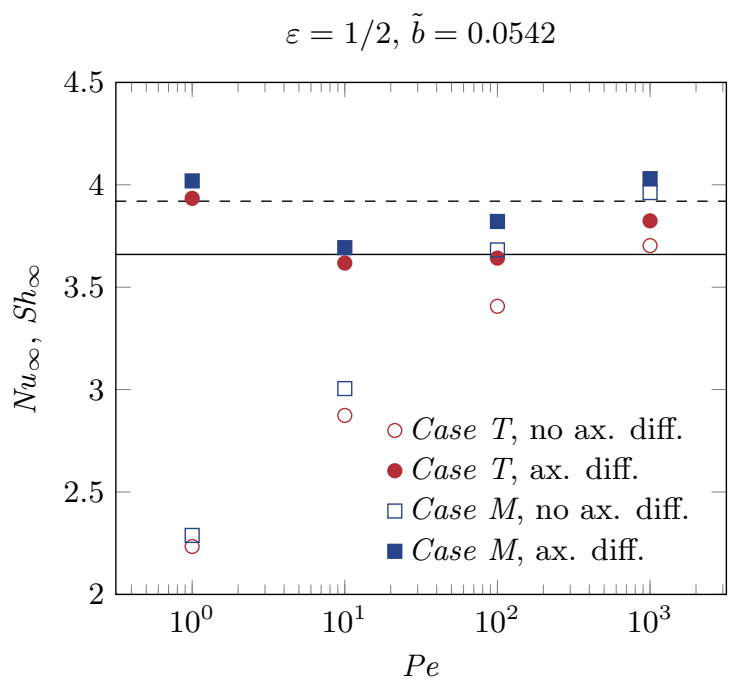

Figure 7.5 - Developed Nusselt and Sherwood number as a function of the Péclet number $P e$. The solid line indicates the classical value for no-slip flow, i.e. $N u_{\infty}, S h_{\infty}=$ 3.66. The dashed line corresponds to the value for a homogeneous slip flow with a constant wall temperature/concentration and having the same slip length $\tilde{b}$ as the superhydrophobic surface, i.e. $N u_{\infty}, S h_{\infty}=3.92$.

become smaller. According to Eq. (7.22), the effective slip length $\tilde{b}$ decreases for increasing $n$, resulting in smaller transverse velocity components $\tilde{u}_{r}$. Note that for large $n$ the average Sherwood number becomes smaller than the classical Graetz-Nusselt solution for no-slip flow. When the size of one no-slip/no-shear unit compared to the tube radius becomes very small $(n \rightarrow \infty)$, the (slip) velocity $\tilde{u}_{b}$ near the wall goes to zero $(\tilde{b} \rightarrow 0)$. In that case advective transport near the wall vanishes, and the wall has, on the scale of the tube radius, a constant wall concentration. One would therefore expect that the Nusselt/Sherwood profiles overlap with the classical Graetz-Nusselt solution [10]. The reason why this is not observed here is because axial diffusion has so far been neglected.

\subsubsection{The significance of axial diffusion - local Péclet number}

To assess the influence of axial conduction/diffusion, the length of the tube could be a proper length scale. In that case $P e_{L}=P e L / D$, which gives, using the numbers given in Table 7.1, for both heat and mass transport a value of at least $P e_{L}=10^{2} \gg 1$. Neglecting axial diffusion seems to be justified, even in the case of heat transfer with a typical Péclet number of $P e=10^{0}$.

However, this compares the ratio of axial convection to axial diffusion on the scale of the tube length. When the average slip velocity $u_{b}$ near the wall becomes 
very small, close to the wall axial diffusion will be faster than convection on the scale of the heterogeneity of the superhydrophobic surface. One can assess the importance of local axial diffusion by looking at the local Péclet number

$$
P e_{b}=\frac{u_{b} L_{s}}{\alpha}=R e_{b} \operatorname{Pr}
$$

Here, $R e_{b}$ is defined as

$$
R e_{b}=\frac{u_{b}}{u_{a v}} \frac{L_{s}}{D} \frac{u_{a v} D}{\nu}=\frac{u_{b}}{u_{a v}} \frac{L_{s}}{D} R e .
$$

In the case of Stokes flow, we can derive that $\tilde{u}_{b}=u_{b} / u_{a v}=4 \tilde{b} /(1+4 \tilde{b})$. Furthermore, we know that $\tilde{b} \approx \pi \varepsilon^{2} / 16 n$ (Eq. (7.22)). For the length of the no-slip area, on which we assume heat/mass exchange takes place in this analysis, we find that $L_{s}=L_{g}(1 / \varepsilon-1)$, where $L_{g}=\varepsilon R / n$. This ultimately gives

$$
P e_{b}=\left[\frac{\pi}{4} \frac{\varepsilon^{2}}{n} /\left(1+\frac{\pi}{4} \frac{\varepsilon^{2}}{n}\right)\right] \frac{1-\varepsilon}{2 n} \operatorname{Re} \operatorname{Pr} .
$$

Because $\tilde{b} \ll 1$ and $\varepsilon^{2} / n \ll 1$, in particular when $n>1$,

$$
P e_{b} \approx \frac{\pi}{8} \frac{\varepsilon^{2}-\varepsilon^{3}}{n^{2}} P e
$$

In this study, $\varepsilon=1 / 2$. This suggests that for heat transfer, $P e_{b} \approx \pi /\left(64 n^{2}\right) P e<1$ for all $n$, because typically $P e \leq 10^{1}$. In this equation we used the effective slip velocity $\tilde{u}_{b}$, which is larger than the actual velocity above the heat exchanging, no-slip area. This makes the required Péclet number $P e$ to let $P e_{b}>1$, suggesting that axial conduction can be neglected, even more stringent. Thus, on average, near the surface diffusion is faster than convection.

For mass transfer, generally $P e>10^{1}$. Then $P e_{b}=\pi /\left(64 n^{2}\right) P e$. For $P e=10^{2}$ this implies that $n>2$ to let $P e_{b}<1$. Besides that, the actual velocity above the mass exchanging surface is larger than the effective slip velocity $u_{b}$. Therefore it seems reasonable to neglect axial diffusion in the case of mass transfer.

Fig. 7.6 shows that axial diffusion indeed conceals the effects of the surface heterogeneity on mass transfer. For $n=1$, fluctuations in the Sherwood number hardly change in size when including axial diffusion: $P e_{b} \gg 1$. For $n=8$ however, for which $P e_{b}<1$, fluctuations are very minor when including axial diffusion. Variations in concentration are smoothed out as a result of axial conduction $[10,24]$, as predicted by Eq. (7.26). That for both $n=1$ and $n=8$ the average Sherwood number increases when including axial diffusion, is because transport is still in the developing regime. In this regime, axial diffusion significantly affects the transport number $[35,36]$. This difference disappears for $P e>10^{2}$ when the 


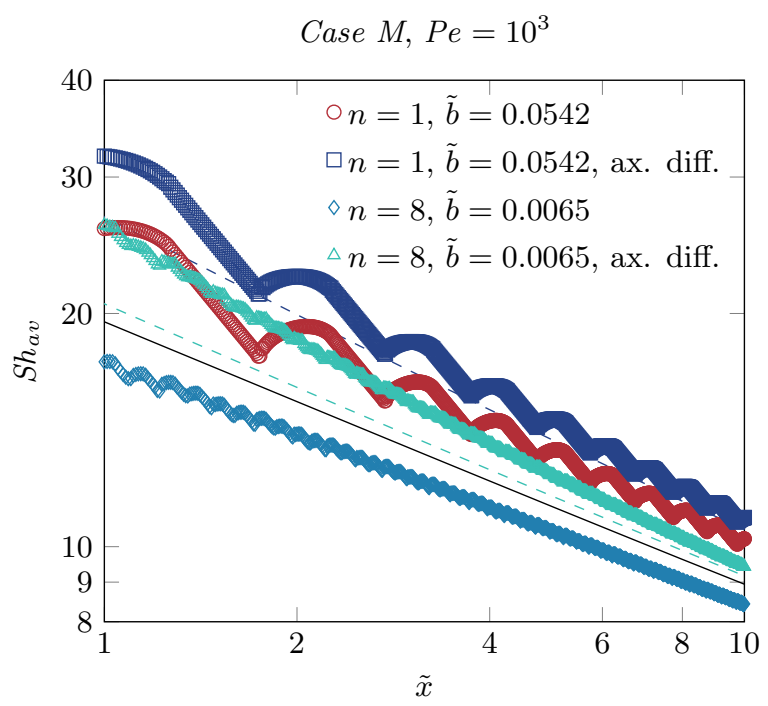

Figure 7.6 - Local average Sherwood number $S h_{a v}$ for $P e=10^{3}$ (with/without axial diffusion) for two different numbers of no-slip/no-shear units $n$ per length $R$. The solid line is the classical Graetz-Nusselt solution. The dashed curves are the Sherwood profiles for a homogeneous slip flow with a constant wall concentration and having the same slip length $\tilde{b}$ as the superhydrophobic surface.

transition regime is approached.

\subsubsection{Influence of axial diffusion on heat/mass transport}

Fig. 7.4 $(a)$ and $(c)$ show the average Nusselt number obtained with and without including axial conduction in the calculations. As comparison of these two plots reveals, axial conduction cannot be neglected for heat transfer when $P e \leq 10^{2}$. For small $P e$ we observe that heat transport is significantly larger than without axial conduction. Also the fluctuations in $N u_{a v}$ become smaller, because axial conduction screens temperature variations along the wall. For $P e>10^{2}$ the influence of axial conduction diminishes, in particular when $\tilde{x}>10$.

For small $\tilde{x}$, even for large $P e$, axial diffusion extends the thermally developing regime. This is in line with the literature $[35,36]$. The developed Nusselt number that is ultimately reached strongly depends on the Péclet number [35, 36]: for $P e=10^{0}, N u_{\infty}=4.03$, which rapidly decreases to the classical value of 3.66 when $P e>10^{1}$. This implies that in the case of heat transfer over superhydrophobic surfaces, for which typically $P e<10^{2}$, axial conduction cannot be neglected. As an approximation, the classical Graetz-Nusselt solutions including axial diffusion could be used to describe heat transport.

Fig. 7.5 shows that the developed Nusselt number $N u_{\infty}$ for flow with axial 
conduction is minimum when $10^{1}<P e<10^{2}$. For low $P e$, the developed Nusselt number corresponds to the classical problem including axial conduction, i.e. $N u_{\infty}=4.03$. Transport near the surface is fully dominated by conduction, and therefore $\Theta \rightarrow 0$ near the adiabatic wall segments. $N u_{\infty}<4.03$ when $10^{1}<P e<10^{2}$ : both diffusion and advection are insufficiently large to level out all temperature variations along the wall. For $P e>10^{2}$ axial convection is so fast, that the adiabatic regions cannot disturb the thermal boundary layer. Near the wall $\Theta \approx 0$. Besides that, for large $P e$ radial advection is large compared to radial diffusion. As a result, $N u_{\infty}$ increases and reaches values larger than the classical value for flow without axial diffusion $\left(N u_{\infty}=3.66\right)$. Finally, the developed Nusselt numbers for flow with and without axial conduction converge towards the same value, as has been observed before [24]. This confirms that only for large Péclet numbers axial diffusion can be neglected.

A similar analysis can be made for mass transport. Fig. 7.4(b) and $(d)$ show that for $P e \geq 10^{2}$ the differences in the average Sherwood numbers $S h_{a v}$ for flows with and without axial diffusion are small, in particular in the developed regime. Inspection of Fig. 7.5 reveals that also the developed Sherwood numbers $S h_{\infty}$ (with/without axial diffusion) converge to the same value. Both observations suggest that in a typical microfluidic system where mass transfer takes place (see Table 7.1), axial diffusion can be neglected. When $P e>10^{2}$, the average and developed Sherwood numbers approach those for a homogeneous slip flow without axial diffusion having a constant wall concentration, and may even exceed them $\left(S h_{\infty}>3.92\right.$ for $\left.P e=10^{3}\right)$. To simplify the description of mass transfer, the solution for homogeneous slip flow with a constant wall concentration could be used as an approximation.

Axial diffusion has greater impact on heat transfer than on mass transport, as comparison of the average and developed Nusselt and Sherwood numbers reveals. Fluctuations are damped more in $N u_{a v}$ than in $S h_{a v}$, as Figs. $7.4(c)$ and $(d)$ show. Fig. 7.5 demonstrates that axial diffusion increases $N u_{\infty}$ relatively more than $S h_{\infty}$. The reason is that heat exchange with the wall takes place where the liquid does not slip. Axial conduction, however, increases the temperature near the adiabatic shear-free wall. Since local slip velocities are high at these wall segments, this considerably enhances convective heat transfer. For mass transfer, the effect of axial diffusion is smaller: it increases the concentration near the impermeable regions. Since these wall segments are non-slippery, here convection is small compared to convection above the mass exchanging no-shear regions.

Note that for all data presented here the number of no-slip/no-shear units per length $R$ is $n=1$, resulting in an effective slip length of $\tilde{b}=0.0542$. With a typical tube diameter of $D=100 \mu \mathrm{m}$ and a porosity of $\varepsilon=1 / 2$, this means that the length of the no-shear segment is $L_{g}=25 \mu \mathrm{m}$. This is large, but experimentally feasible [37]. Depending on how $\varepsilon$ or $n$ are changed, the slip length $\tilde{b}$ may decrease. 
This affects the value of the Nusselt and Sherwood numbers found in microfluidic systems. When $n \rightarrow \infty$ and thus $\tilde{b} \rightarrow 0$, fluctuations in Nusselt/Sherwood numbers arising from the heterogeneity of the superhydrophobic wall disappear. In that case, the tube wall can be considered as non-slippery and as having a constant temperature/concentration.

\subsection{Conclusion}

In this study we described the Graetz-Nusselt problem for heat and mass transport over slippery, superhydrophobic surfaces. The results revealed that the performance of heat and mass transfer can be very distinct. The subtle differences in boundary conditions arising from the heterogeneous nature of the wall should therefore be taken into account.

In the case of thermal transport, the no-slip wall has a constant wall temperature and the no-shear wall is adiabatic. For mass transfer, the no-shear wall has a constant wall concentration and the no-slip wall is impermeable. These differences make, on average, mass transfer more efficient than thermal transport, as expressed by the average Nusselt and Sherwood numbers. Only in the case of mass transfer do the locations of hydrodynamic slip and mass exchange coincide. Advection near the mass exchanging wall segments is therefore larger than near the heat exchanging regions. Also the direction of radial momentum transport is reversed, leading to a compression of the concentration/temperature boundary layer at the beginning of these segments for mass transport, and at the end of these segments for heat transfer. Increasing the Péclet number $P e$ makes the difference in performance between heat and mass transport more pronounced.

Although it may simplify the description of heat/mass transport, axial diffusion cannot always be neglected. Our results show that for $P e<10^{2}$, which is typically the case for heat transfer in superhydrophobic microfluidic systems, axial conduction should be taken into account. This prevents underestimation of the length of the thermally developing regime and the value of the developed Nusselt number. For $P e \geq 10^{2}$, which are typical numbers for mass transport, axial conduction has only a minor influence on the rate of mass transport and can be ignored.

\section{References}

[1] L. Graetz, Über die Wärmeleitungsfähigkeit von Flüssigkeiten, Ann. Phys. 254, 79 (1882).

[2] L. Graetz, Über die Wärmeleitungsfähigkeit von Flüssigkeiten, Ann. Phys. 261, 337 (1885). 
[3] W. Nusselt, Die Abhängigkeit der Wärmeübergangszahl von der Rohrlänge, Z. Verein. Deutsch. Ing. 54, 1154 (1910).

[4] R. B. Bird, W. E. Stewart, and E. N. Lightfoot, Transport phenomena, 2nd ed. (John Wiley \& Sons, New York, 2007).

[5] A. S. Haase, S. J. Chapman, P. A. Tsai, D. Lohse, and R. G. H. Lammertink, The Graetz-Nusselt problem extended to continuum flows with finite slip, J. Fluid Mech. 764, R3 (2015).

[6] E. Lauga, M. P. Brenner, and H. A. Stone, in Springer handbook of experimental fluid mechanics, edited by C. Tropea, A. L. Yarin, and J. F. Foss (Springer, Berlin, 2007), pp. 1219-1240.

[7] J. P. Rothstein, Slip on superhydrophobic surfaces, Annu. Rev. Fluid Mech. 42, 89 (2010).

[8] L. Bocquet and J.-L. Barrat, Flow boundary conditions from nano- to microscales, Soft Matter 3, 685 (2007).

[9] K. H. Keller and T. R. Stein, A two-dimensional analysis of porous membrane transport, Math. Biosci. 1, 421 (1967).

[10] N. M. Juhasz and W. M. Deen, Effect of local Peclet number on mass transfer to a heterogeneous surface, Ind. Eng. Chem. Res. 30, 556 (1991).

[11] N. M. Juhasz and W. M. Deen, Mass transfer in a tube with wall flux confined to evenly spaced discrete areas, Chem. Eng. Sci. 48, 1745 (1993).

[12] S. C. Ling, Heat transfer from a small isothermal spanwise strip on an insulated boundary, Journal of Heat Transfer 85, 230 (1963).

[13] M. A. Lévêque, Les lois de la transmission de chaleur par convection, Ann. Mines, Mem., Ser. 13, 201 (1928).

[14] R. C. Ackerberg, R. D. Patel, and S. K. Gupta, The heat/mass transfer to a finite strip at small Péclet numbers, J. Fluid Mech. 86, 49 (1978).

[15] C. G. Phillips, Heat and mass transfer from a film into steady shear flow, Q. J. Mech. Appl. Math. 43, 135 (1990).

[16] H. A. Stone, Heat/mass transfer from surface films to shear flows at arbitrary Peclet numbers, Phys. Fluids A 1, 1112 (1989).

[17] K.-K. Tio and S. S. Sadhal, Boundary conditions for Stokes flows near a porous membrane, Appl. Sci. Res. 52, 1 (1994).

[18] C. Jia, K. Shing, and Y. C. Yortsos, Advective mass transfer from stationary sources in porous media, Water Resour. Res. 35, 3239 (1999).

[19] S. Veran, Y. Aspa, and M. Quintard, Effective boundary conditions for rough reactive walls in laminar boundary layers, Int. J. Heat Mass Transfer 52, 3712 (2009).

[20] P. N. Shah and E. S. G. Shaqfeh, Heat/Mass transport in shear flow over a heterogeneous surface with first order surface reactive domains, J. Fluid Mech. 782, 260 (2015).

[21] A. S. Haase, E. Karatay, P. A. Tsai, and R. G. H. Lammertink, Momentum 
and mass transport over a bubble mattress: the influence of interface geometry, Soft Matter 9, 8949 (2013).

[22] D. Maynes, B. W. Webb, and J. Davies, Thermal transport in a microchannel exhibiting ultrahydrophobic microribs maintained at constant temperature, J. Heat Transfer 130, 022402 (2008).

[23] D. Maynes, B. W. Webb, J. Crockett, and V. Solovjov, Analysis of laminar slip-flow thermal transport in microchannels with transverse rib and cavity structured superhydrophobic walls at constant heat flux, J. Heat Transfer 135, 021701 (2012).

[24] A. Cowley, D. Maynes, and J. Crockett, Effective temperature jump length and influence of axial conduction for thermal transport in superhydrophobic channels, Int. J. Heat Mass Transfer 79, 573 (2014).

[25] R. Enright, M. Hodes, T. Salamon, and Y. Muzychka, Isoflux Nusselt number and slip length formulae for superhydrophobic microchannels, J. Heat Transfer 136, 012402 (2013).

[26] D. Maynes and J. Crockett, Apparent temperature jump and thermal transport in channels with streamwise rib and cavity featured superhydrophobic walls at constant heat flux, J. Heat Transfer 136, 011701 (2014).

[27] C.-O. Ng and C. Y. Wang, Temperature jump coefficient for superhydrophobic surfaces, J. Heat Transfer 136, 064501 (2014).

[28] L. Steigerwalt Lam, C. Melnick, M. Hodes, G. Ziskind, and R. Enright, Nusselt numbers for thermally developing couette flow with hydrodynamic and thermal slip, J. Heat Transfer 136, 051703 (2014).

[29] D. Moreira and P. R. Bandaru, Thermal transport in laminar flow over superhydrophobic surfaces, utilizing an effective medium approach, Phys. Fluids 27, 052001 (2015).

[30] J. S. Wexler, A. Grosskopf, M. Chow, Y. Fan, I. Jacobi, and H. A. Stone, Robust liquid-infused surfaces through patterned wettability, Soft Matter 11, 5023 (2015).

[31] C. L. M. H. Navier, Mémoire sur les lois du mouvement des fluids, Mem. Acad. Sci. Int. Fr. 6, 389 (1823).

[32] A. M. J. Davis and E. Lauga, Geometric transition in friction for flow over a bubble mattress, Phys. Fluids 21, 011701 (2009).

[33] R. F. Barron, X. Wang, T. A. Ameel, and R. O. Warrington, The Graetz problem extended to slip-flow, Int. J. Heat Mass Transfer 40, 1817 (1997).

[34] F. Ezquerra Larrodé, C. Housiadas, and Y. Drossinos, Slip-flow heat transfer in circular tubes, Int. J. Heat Mass Transfer 43, 2669 (2000).

[35] D. K. Hennecke, Heat transfer by Hagen-Poiseuille flow in the thermal development region with axial conduction, Wärme Stoffübertrag. 1, 177 (1968).

[36] R. Shah and A. London, Laminar flow forced convection in ducts (Academic 
Press, New York, 1978), pp. 78-152.

[37] E. Karatay, A. S. Haase, C. W. Visser, C. Sun, D. Lohse, P. A. Tsai, and R. G. H. Lammertink, Control of slippage with tunable bubble mattresses, P. Natl Acad. Sci. USA 110, 8422 (2013). 




\title{
CHAPTER 8
}

\section{Desalination by electrodialysis using a stack of ion-selective hydrogels on a microfluidic device}

\begin{abstract}
We report a novel approach for the separation of charged species using anion- and cation-exchange hydrogels in a microfluidic device. The capillary line pinning technique we applied enables in situ fabrication of alternating anion- and cation-exchange hydrogels that are placed in confined compartments. Adjacent enriched and depleted streams were obtained in continuous flow when a potential difference was applied over the hydrogel stack. The desalination performance of the microchip was demonstrated at different salt concentrations $(0.01-1 \mathrm{mM}$ sodium chloride), potentials $(10-100 \mathrm{~V})$, current densities $\left(3-7 \mathrm{~A} / \mathrm{m}^{2}\right)$ and liquid flow rates $(0-5 \mu \mathrm{L} / \mathrm{min})$. We show that the microchip was able to remove approximately $75 \%$ of the salt initially present in the depleted outlet streams for an inlet stream concentration of $1 \mathrm{mM}$ sodium chloride. Besides desalination, the microchip allows the visualisation of ion transport in the ion-selective hydrogels. It can therefore be used to study the interplay of transport phenomena at the electrolyte-hydrogel interface during the desalination process.
\end{abstract}

This chapter describes a joint work which has been accepted for publication as B. Gümüşcü*, A.S. Haase*, A.M. Benneker*, M.A. Hempenius, A. van den Berg, R.G.H. Lammertink and J.C.T. Eijkel, Desalination by electrodialysis using a stack of patterned ionselective hydrogels on a microfluidic device, Adv. Func. Mater. (2016). 


\subsection{Introduction}

Membrane stacks are widely used for electrodialysis (ED) and reverse electrodialysis (RED) in macroscale systems [1-3]. Alternating cation- (CEM) and anion-selective membranes (AEM) are stacked with spacers to allow a flowing electrolyte solution between the membranes. When an electric field is applied to this system, the anions in the electrolyte solution will migrate towards the anode, while the cations migrate towards the cathode. However, anions and cations will eventually be blocked by the CEM and AEM, respectively, resulting in the formation of alternatingly depleted and enriched flow streams. When the applied electric field strength is increased, the ion transport rate and therefore the current in the system will rise, until a critical point is reached. Beyond this point, the system enters the well-known limiting current regime [4], where the current no longer increases with electric field strength and the energy efficiency of the ion separation process is reduced. In this regime, a boundary depletion layer is formed in which the major resistance for ion transport is located. When increasing the electric field strength further, or in the case of membrane discharge due to the developing $p \mathrm{H}$ in the membrane, the overlimiting current regime can be reached, resulting in the transport of additional ions obtained through either water splitting or hydrodynamic effects $[5,6]$. Water splitting can also lead to a developing $p \mathrm{H}$ profile in the membrane that can give rise to a change in membrane charge density. The hydrodynamic and ion transport phenomena can result in a loss of performance for macroscale ED systems [7-9].

Thanks to the advances in microfabrication techniques, ion-selective materials have been integrated in fluidic platforms, increasing the number of experimental investigations of the aforementioned phenomena [10-16]. Microfluidic desalination systems have been applied for two purposes. First, microfluidics has been used for downscaling the electrodialysis process in order to obtain more insight into the ion transport phenomena that occur at the microscale [17]. Kim et al. used two microchannels separated by a Nafion membrane, which was fabricated using micro-stamping, to observe multiple vortical flows inside the ion concentration polarisation layer [14]. Kwak et al. fabricated a polydimethylsiloxane (PDMS) microchip consisting of externally mounted commercial ion-exchange membranes for visualisation of concentration and liquid flow profiles in the Ohmic, limiting, and overlimiting current regimes [15]. Second, microfluidics has been used to manufacture miniaturised electrodialysis devices for application purposes. Recently, in situ fabrication of (hybrid) membranes by photolithography in glass microchips has been demonstrated for DNA preconcentration [12] and for microscale $p \mathrm{H}$ regulation by splitting water [13]. In another work, solvent evaporation was used for in situ fabrication of ion-selective membranes in a PDMS microchip for inducing ion concentration polarisation in order to preconcentrate proteins [16]. 
The aforementioned methods for incorporation of membranes in microfluidic systems pose challenges in terms of robustness, consistency and ease of fabrication. For example, photolithography techniques bring alignment problems, while evaporation bears the risk of damaging the membranes. Integration of pre-fabricated membranes in microfluidic chips can lead to liquid leakage [18]. In situ fabrication of membranes in microchips is an alternative, but is difficult when using, for instance, interfacial polymerisation [19]. Hydrogels form an alternative to membranes, and have been successfully incorporated in microfluidic devices in the past $[13,20]$. Previously, we introduced the capillary line pinning technique for controllable patterning of hydrogels in microchips [21]. Hydrogels are therefore promising candidates for studying the hydrodynamic and ion transport phenomena in microfluidic devices, as they provide an ion-selective and hydrophilic matrix, which is versatile, inexpensive and tailorable [13, 20].

In this work, we present a microfluidic device consisting of a stack of alternatingly patterned and oppositely charged hydrogel patches fabricated by capillary line pinning for performing electrodialysis. To the best of our knowledge, the use of such a platform has not been demonstrated or investigated before. As a proof of principle, periodic hydrogel patches were used to obtain alternatingly diluted and enriched parallel streams under different flow rates and applied potential differences. By using a negatively charged fluorescent dye, this platform also allowed us to study ion transport through the hydrogels as well as salt concentration fluctuations and ion concentration polarisation (ICP) in the microchannels. The ability to visualise charge transport in the hydrogels helps to obtain a better understanding of the hydrodynamic and ion transport phenomena that occur in ED and RED systems. Besides that, PDMS microchips with in situ patterned hydrogels enable the fabrication of versatile ion separation platforms.

\subsection{Experimental}

\subsubsection{Microchip and hydrogel fabrication}

The SU-8 masters of the polydimethylsiloxane (PDMS) microchips, see Fig. 8.1(a), were fabricated in the $\mathrm{MESA}^{+}$cleanroom facility at the University of Twente. The masters consisted of two layers of SU-8. The width of the microchannels and the pillars was $700 \mu \mathrm{m}$ and $1720 \mu \mathrm{m}$, respectively. The first layer contained the pillar layout and its thickness was measured as $67.5 \mu \mathrm{m}$ (Bruker Dektak, Germany). The second layer contained both pillar and capillary barrier layouts with a width of $2.0 \mu \mathrm{m}$ and a thickness of $7.5 \mu \mathrm{m}$ (see Fig. 8.1(b) and the appendix/Fig. 8.8).

The microchips were fabricated by assembling a PDMS layer with a microscope slide. PDMS precursor was prepared, degassed, poured onto the master and cured. The cured PDMS was peeled off from the master and treated with oxygen plasma 


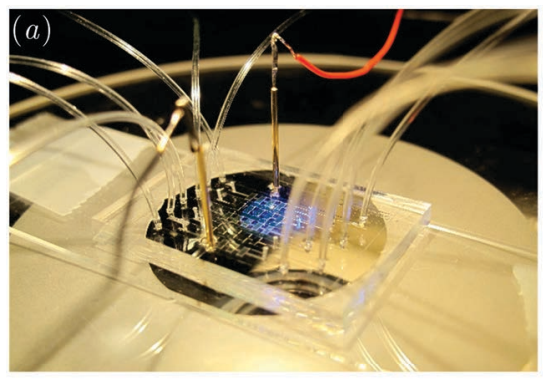

(c) PDMS pillar (b) $\mathrm{NaCl}$ solution

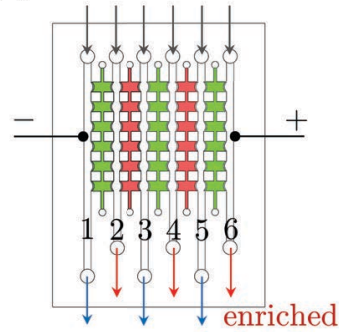

depleted

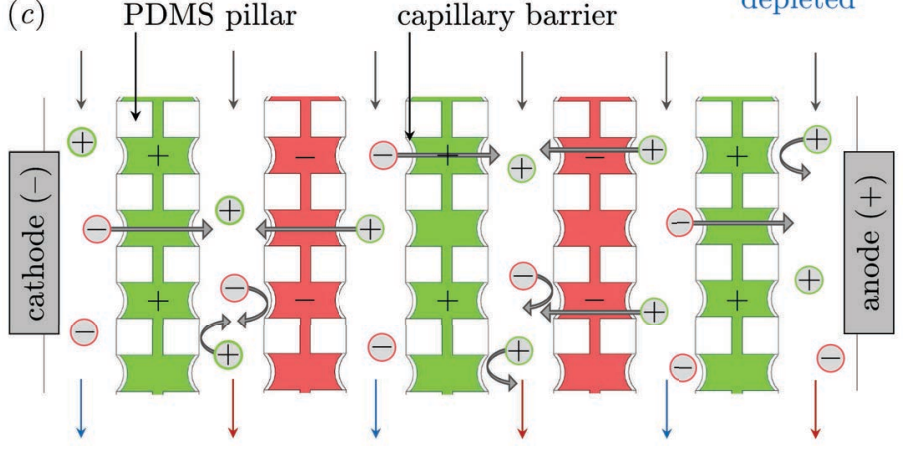

Figure 8.1 - (a) Image of the assembled microchip. (b) Schematic illustration of the membrane stack consisting of alternating rows of anion-exchange hydrogels (AEHs) and cation-exchange hydrogels (CEHs). (c) Schematic representation of the desalination process.

to ensure irreversible bonding with the microscope slide. All microchannels, pillars, capillary barriers, inlets and outlets were structured in the PDMS layer. Fig. 8.1(a) shows an assembled microchip with PDMS pillars and capillary barriers. All hydrogel compartments (each with a size of $200 \times 500 \mu \mathrm{m}$ ) in a specific row are connected with each other via microchannels.

All hydrogel solutions (each with a volume of $500 \mu \mathrm{L}$ ) were degassed at $7 \mathrm{kPa}$ vacuum for $15 \mathrm{~min}$ immediately prior to use. To avoid the presence of dust particles, all hydrogel precursors were prepared in a nitrogen environment in a flow hood by blending $20 \% \mathrm{v} / \mathrm{v}$ of acrylamide/bis (19:1) (BioRad), $15 \% \mathrm{w} / \mathrm{v}$ N,N'-bis(2-hydroxyethyl)ethylenediamine (bis, Sigma-Aldrich), $10 \% \mathrm{w} / \mathrm{v}$ of 2,2dimethoxy-2-phenylacetophenone (DMPA, Invitrogen) and $5 \% \mathrm{w} / \mathrm{v}$ of ammonium persulfate solutions (Invitrogen). For the anion-exchange hydrogels (AEH), 1\% v/v [2-(methacryloyloxy)ethyl]trimethylammonium chloride solution (METC, SigmaAldrich), and for the cation-exchange hydrogels (CEH) 1\% w/v 3-sulfopropyl acrylate potassium salt (SPAP, Sigma-Aldrich) was added to the acrylamide/bis mixture. As the crosslinking reaction is inhibited by oxygen, the patterning and polymerisation processes were performed in a nitrogen environment. 

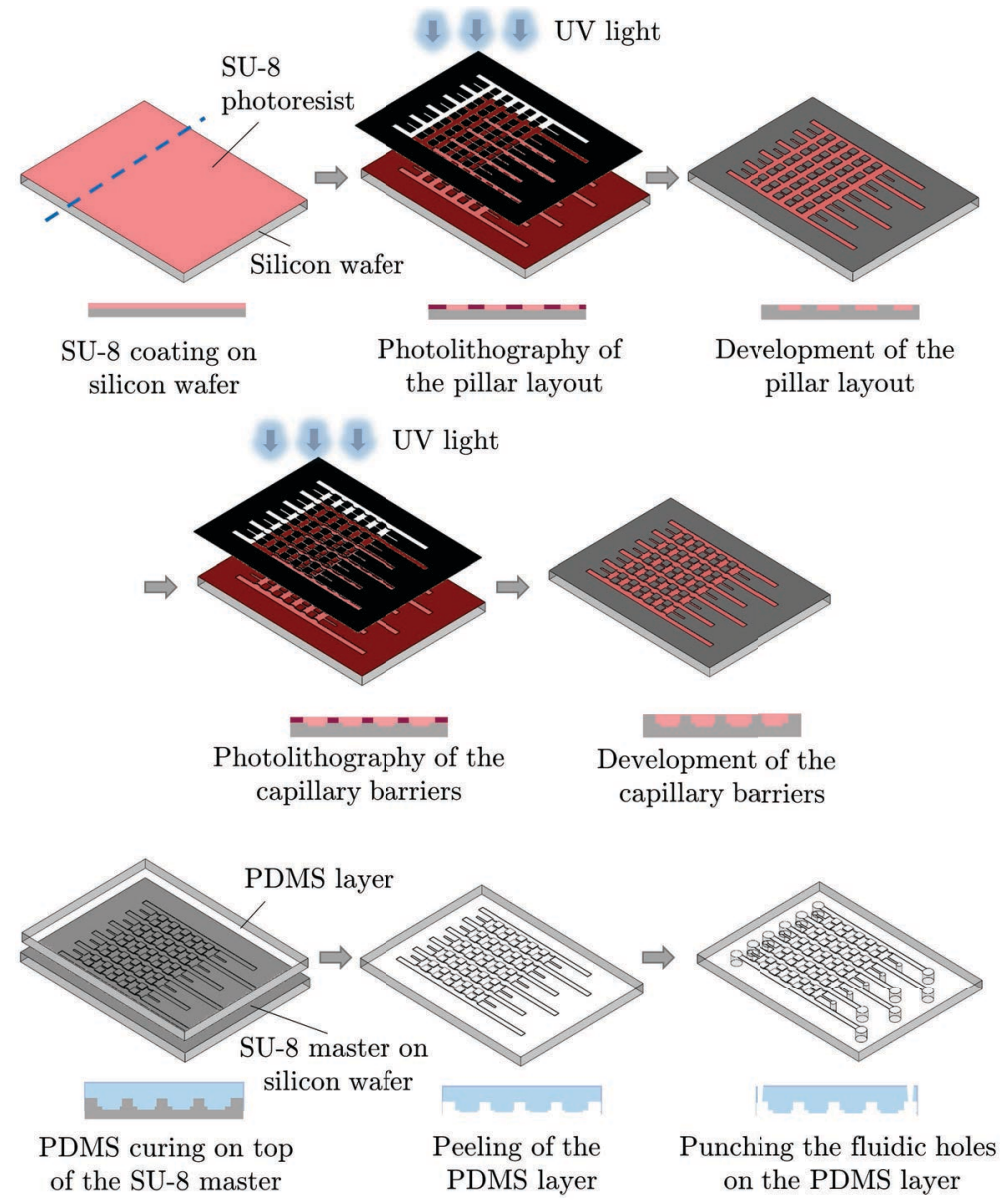

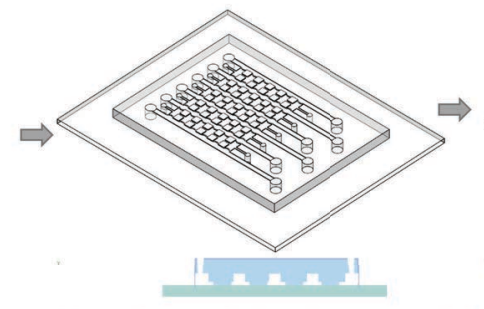

Microchip and glass layer assembly by $\mathrm{O}_{2}$ plasma treatment

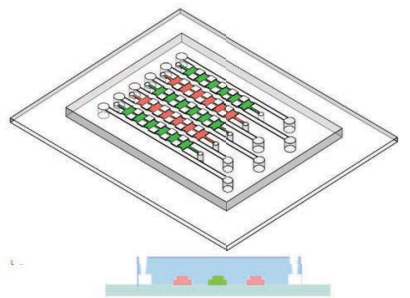

Patterning and curing the charged hydrogels

Figure 8.2 - Photolithography, soft lithography and chip assembly procedures. The schematics on the first two lines illustrate the photolithography process. The schematics on the third line present the soft lithography process. The fourth line depicts the chip assembly and patterning procedure. The blue dashed line indicates the position of the cross sectional area. 
Fig. 8.2 outlines the microchip fabrication and hydrogel patterning process. A detailed explanation of the capillary pinning technique has been reported by Gümüşcü et al. [21]. After the degassing process, approximately $0.5 \mu \mathrm{L}$ of the $\mathrm{AEH}$ and $\mathrm{CEH}$ solutions was alternatingly pipetted into the parallel rows by capillary action. The hydrogel precursor remained contained between the PDMS pillars due to capillary pinning at the barriers. The patterned precursors were subsequently exposed to UV light at $400 \mathrm{~mW} / \mathrm{cm}^{2}$ for $3 \mathrm{~min}$. Microchips containing CEHs and $\mathrm{AEHs}$ were immersed in a $0.1 \mathrm{mM} \mathrm{NaCl}$ solution at room temperature. Each microchip contained six microchannels, which were connected to each other via the alternating rows of AEHs and CEHs as shown in Fig. 8.1(b).

\subsubsection{Characterisation of hydrogels}

Scanning electron microscopy. Thin films of polymerised hydrogels were casted on a glass surface and freeze-dried for 2 days. Samples were kept in a vacuum chamber overnight and subsequently coated with a $5 \mathrm{~nm}$ layer of chromium using an E5000 sputter coater (Bio-Rad). Scanning electron microscopy (SEM) images were taken using a FEI Sirion HR-SEM.

FTIR and XPS. Fourier transform infrared spectroscopy (FTIR) spectra were measured with a Bruker ALPHA (Bruker Optics) using an attenuated total reflectance attachment. Polymerised hydrogels were freeze-dried for 2 days in Eppendorf tubes and kept in a vacuum chamber overnight. Dried samples were directly analysed without any further sample preparation. For X-ray photoelectron spectroscopy (XPS), 1 g of both polymerised AEH and CEH was freeze-dried for 2 days in Eppendorf tubes and kept in a vacuum chamber overnight. Dried samples were analysed using a Quantera SXM (Physical Electronics).

Ion exchange capacity. The ion exchange capacity (IEC) is the amount of charged groups in the hydrogel and is measured by titration [22]. For the titration of the $\mathrm{AEH}, 0.7 \mathrm{~g}$ of the hydrogel was immersed in a $3 \mathrm{M} \mathrm{NaCl}$ solution (Sigma-Aldrich) for 3 days at room temperature to exchange all counter ions in the $\mathrm{AEH}$ for $\mathrm{Cl}^{-}$. After rinsing it with deionised water, the chloride ions were replaced by sulfate ions by immersing the hydrogel into a $1.5 \mathrm{M} \mathrm{Na}_{2} \mathrm{SO}_{4}$ solution for 3 hours. The $\mathrm{Na}_{2} \mathrm{SO}_{4}$ solution was refreshed every hour of the experiment and the collected solutions were titrated with a $0.1 \mathrm{M} \mathrm{AgNO}_{3}$ solution. The required volume of $\mathrm{AgNO}_{3}$ was recorded. The hydrogel was dried in a vacuum oven at $60^{\circ} \mathrm{C}$ for 48 hours and its dry weight was recorded for IEC calculations. A similar procedure was followed to determine the IEC of the $\mathrm{CEH}$. $0.7 \mathrm{~g}$ of the $\mathrm{CEH}$ was immersed in a $1 \mathrm{M} \mathrm{HCl}$ solution to exchange all counter ions for $\mathrm{H}^{+}$. The hydrogel was then rinsed with deionised water and immersed in a $2 \mathrm{M} \mathrm{NaCl}$ solution for 3 hours to replace the hydrogen ions for sodium ions. The $\mathrm{NaCl}$ solution was refreshed every hour and the collected solutions were titrated with a $0.1 \mathrm{M} \mathrm{NaOH}$ solution. The hydrogel was dried in a vacuum oven. All experiments were repeated three times. 
Water swelling. Water swelling of the hydrogels was determined by immersion in deionised water and subsequent drying in a vacuum oven. The degree of water swelling $S$ was calculated using the following equation: $S=\left(W_{s}-W_{d}\right) / W_{d}$. Here, $W_{s}$ and $W_{d}$ represent the weight $W$ of the swollen $(s)$ hydrogels and the dried $(d)$ hydrogels. Also the swelling degree of the polymerised hydrogels was tested: 2 $\mathrm{mL}$ of bulk hydrogel after polymerisation was immersed in $50 \mathrm{~mL}$ deionised water for 72 hours, and patterned hydrogels on chip were immersed in deionised water for 16 hours.

Permselectivity. The permselectivity determines the capability of the hydrogel to discriminate between anions and cations. An in-house built two-compartment plexiglass cell was used for the permselectivity experiments. The charged bulk hydrogel was located in between the two compartments. By using a peristaltic pump, one of the compartments was filled with a $0.1 \mathrm{M} \mathrm{NaCl}$ solution, while the other compartment was filled with a $0.5 \mathrm{M} \mathrm{NaCl}$ solution. Both solutions were recirculated continuously during the measurement and kept at $25^{\circ} \mathrm{C}$ using a thermostatic bath. Two reference calomel electrodes were used to measure the electric potential over the hydrogel by connecting the system to a potentiostat (Metrohm Autolab PGSTAT302N) for monitoring the changes in voltage over the hydrogel. The apparent permselectivity $\alpha=\Delta V_{\text {meas }} / \Delta V_{\text {theor }}$ was calculated for both $\mathrm{AEH}$ and $\mathrm{CEH}$ separately using the ratio of the measured potential difference $\Delta V_{\text {meas }}$ over the theoretical potential difference $\Delta V_{\text {theor }}$ for an ideal permselective membrane. The theoretical potential difference was calculated using the Nernst equation $\Delta V_{\text {theor }}=(R T) /(z F) \ln \left(c_{1} \gamma_{1} / c_{2} \gamma_{2}\right)$. Here, $R$ is the gas constant, $T$ the temperature, $z$ the electrochemical valence and $F$ the Faraday constant [23]. The salt concentrations and activity coefficients of the two solutions are indicated by $c_{i}$ and $\gamma_{i}$, where $\gamma_{i}$ denotes the mean activity coefficient for an aqueous $\mathrm{NaCl}$ solution at $25^{\circ} \mathrm{C}$ as obtained from [24].

Membrane resistivity. The area resistances of both $\mathrm{AEH}$ and $\mathrm{CEH}$ were measured using an in-house built six-compartment plexiglass cell. In the experiments, a $0.1 \mathrm{M} \mathrm{NaCl}$ solution was used and the voltage drop $\Delta V$ over the hydrogel was recorded for an applied current density $I / A$. Here, $I$ is the current through the membrane with area $A$. The resistivity $R_{\rho}$ of each hydrogel, after baseline correction by performing a blank run, was given by the slope of the potential difference versus current density curve. Thus, $R_{\rho}=(A \Delta V) /(I d), d$ being the thickness of the hydrogel.

Electrodialysis experiments. The desalination performance of the microchip was investigated using solutions with different $\mathrm{NaCl}$ concentrations (0.01, 0.1 and $1 \mathrm{mM}$ ) containing $5 \mu \mathrm{M}$ Alexa Fluor 488 Cadaverine (ThermoFisher Scientific), which is a negatively charged fluorescent dye. This dye was utilised to determine the local ion concentration in the microchannels qualitatively. Gold coated copper electrodes were placed in the outer two microchannels adjacent to the hydrogels. 
Table 8.1 - Bulk material characteristics of cross-linked METC and SPAP polymers, with the $95 \%$ confidence interval of the mean indicated. Except for the resistivity and permselectivity, all measurements have been repeated three times.

\begin{tabular}{lll}
\hline & AEH (METC) & CEH (SPAP) \\
\hline Swelling $S$ in deionised water $(\%)$ & $460 \pm 10$ & $450 \pm 10$ \\
Ion exchange capacity IEC $\left(\mathrm{mmol} / \mathrm{g}_{\text {dry }}\right)$ & $1.95 \pm 0.45$ & $0.64 \pm 0.09$ \\
Charge density CD (mmol/g $\left.\mathrm{g}_{\mathrm{H}_{2} \mathrm{O}}\right)$ & $0.43 \pm 0.10$ & $0.14 \pm 0.02$ \\
Resistivity $R_{\rho}(\Omega \mathrm{cm})$ (single meas.) & 310 & 470 \\
Permselectivity $\alpha(\%)$ (single meas.) & 29 & 26 \\
\hline
\end{tabular}

An electrical potential or current was applied by a Keithley 2450 Source Meter, controlled by a home-built LabVIEW program. The fluorescent dye was visualised using a Hamamatsu ORCA-Flash4.0 LT camera mounted on an inverted Zeiss Axiovert 40 MAT microscope. The liquid flow was supplied to the microchip using six Harvard Picoplus programmable syringe pumps and six Braun Omnifix-F $1 \mathrm{~mL}$ syringes. The samples were collected separately at the outlet of each microchannel. The conductivity of the samples was measured using a potentiostat (BioLogic SP300) combined with an in-house made interdigitated electrode sensor. By using a calibration curve, the outlet stream salt concentrations could be obtained from the measured impedance (see the appendix/Fig. 8.9).

\subsection{Results and discussion}

\subsubsection{Characterisation of hydrogels}

In the appendix/Fig. 8.10, the molecular structures of the charged monomers (METC and SPAP) photopolymerised with acrylamide and bis are shown. The free radicals yielded by DMPA started the crosslinking process by randomly associating with acrylamide, bis and the METC or SPAP monomers or polymer chains, which eventually became polymerised in the illuminated region. The photopolymerisation process has been explained in detail in our previous work [25]. The SEM images indicate that the hydrogels obtained after photopolymerisation were homogeneous (see the appendix/Fig. 8.11).

METC and SPAP are suitable monomers to obtain charge selective interfaces for electrodialysis, because they permanently have a positive and negative charge density, respectively, which is $p \mathrm{H}$ independent in the range of $p \mathrm{H} \mathrm{2-12} \mathrm{[26,} \mathrm{27].}$ In addition, the hydrolytic stability of METC and SPAP polymers enables charge based separations in electrodialysis microchips [26, 27]. In some previous studies, both charged monomers have shown to be super-absorbents, which results in strong swelling behaviour $[28,29]$. In this work, the swelling behaviour was suppressed by 


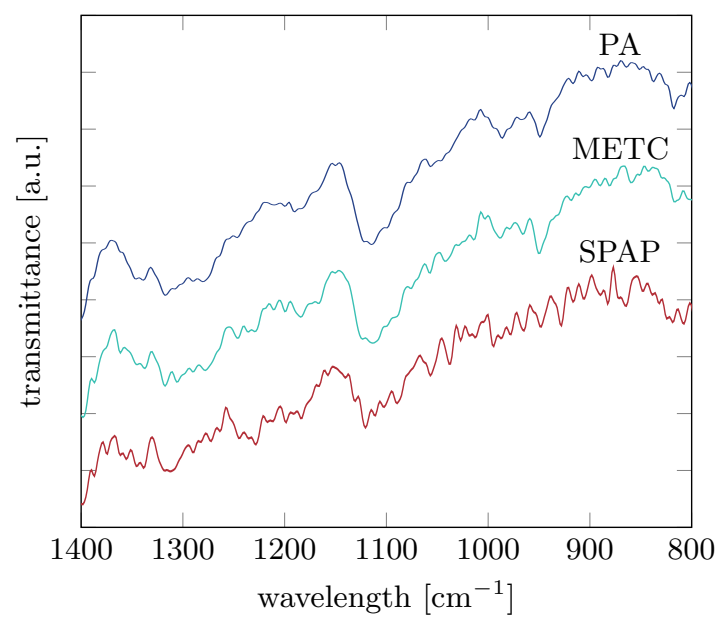

Figure 8.3 - FTIR spectra of polyacrylamide (PA), METC + PA (anion exchange hydrogel) and SPAP + PA (cation exchange hydrogel) are shown.

increasing the cross-linking density and the amount of uncharged monomers. We measured the water swelling of the bulk METC and SPAP polymers as $460 \pm 10 \%$ for METC and as $450 \pm 10 \%$ for SPAP after drying the bulk materials in a vacuum oven (Table 8.1). The high swelling degree indicates the ability of the hydrogels to absorb water in high contents, which is related to the hydrophilic nature of the polyacrylamide backbone. Note that directly after photopolymerisation, the hydrogels already have a high water content. By immersing the hydrogels in deionised water directly after photopolymerisation, the additional swelling of the bulk hydrogels was measured to be $15 \%$ and $16 \%$ for $\mathrm{AEH}$ and $\mathrm{CEH}$, respectively. Both hydrogels showed identical swelling behaviour in the microchip as well, as shown in the appendix/Fig. 8.8. In addition, immersed hydrogels are expected to swell less in salt solutions than in deionised water [30]. The reason is that hydrogel swelling decreases with increasing ion concentration, as the charged side groups present in the hydrogels are increasingly screened by the ions in the salt solution $[31,32]$.

The presence of cross-linker and SPAP or METC was verified using FTIR. The FTIR spectra shown in Fig. 8.3 indicate the presence of SPAP sulfonate groups from $\mathrm{S}=\mathrm{O}$ stretching bands at 982 and $1043 \mathrm{~cm}^{-1}$ and $\mathrm{SO}_{2}$ symmetric stretching absorption at $1178 \mathrm{~cm}^{-1}$ [33]. A relatively weak absorption at 1170 $\mathrm{cm}^{-1}$ was ascribed to $\mathrm{N}-\mathrm{CH}_{3}$ stretching of METC repeat units [33]. $\mathrm{C}-\mathrm{N}$ stretching absorptions in general are known to be weak [33, 34]. We measured the FTIR spectra of the corresponding monomers to confirm the location of the peaks observed in the polymers (see the appendix/Fig. 8.12). 
Table 8.2 - XPS results for polyacrylamide (PA), the positively charged hydrogel (PA + METC) and the negatively charged hydrogel (PA + SPAP).

\begin{tabular}{lcccccc}
\hline Element (\%) & $\mathrm{C}(1 \mathrm{~s})$ & $\mathrm{N}(1 \mathrm{~s})$ & $\mathrm{O}(1 \mathrm{~s})$ & $\mathrm{S}(2 \mathrm{p})$ & $\mathrm{Cl}(2 \mathrm{p})$ & $\mathrm{K}(2 \mathrm{p})$ \\
& $283 \mathrm{eV}$ & $399 \mathrm{eV}$ & $529 \mathrm{eV}$ & $164 \mathrm{eV}$ & $200 \mathrm{eV}$ & $293 \mathrm{eV}$ \\
\hline $\mathrm{PA}$ & 64.6 & 16.3 & 18.7 & - & - & - \\
$\mathrm{PA}+\mathrm{METC}$ & 64.4 & 16.3 & 20.1 & - & 0.5 & - \\
$\mathrm{PA}+\mathrm{SPAP}$ & 62.1 & 16.1 & 20.3 & 1.2 & - & 0.1 \\
\hline
\end{tabular}

We also analysed the chemical composition of the hydrogels using XPS to verify the presence of either METC or SPAP. The atomic ratios of carbon, nitrogen, oxygen, sulphur, chlorine and potassium are given in Table 8.2. METC and SPAP polymers were compared with PA polymer for the evaluation of the atomic percentage changes. For the METC polymer, the amount of carbon atoms remained the same, despite the fact a slight increase was expected [34]. In agreement with the expected composition, the nitrogen atom content remained the same and oxygen content increased. The detection of chloride clearly indicates that charged $\mathrm{N}^{+}$groups were present $[35,36]$. In the SPAP hydrogel the content of carbon atoms decreased, which could be an experimental artefact. On the other hand, as expected, the amount of nitrogen atoms remained constant. The increased amount of oxygen atoms can be attributed to the high oxygen content of the SPAP monomer [34]. The presence of sulfonate groups was validated by the detection of sulphur and potassium atoms. However, potassium was present in a lower amount than expected. These atoms might be out of the scanning range due to the orientation of the charged groups on the polymer film.

Table 8.1 shows some material characteristics of the anion-exchange (METC) and cation-exchange (SPAP) hydrogels. Compared to commercially available ion exchange membranes [37, 38], METC exhibited a comparable and SPAP a low ion exchange capacity. When considering the charge density, we found that both hydrogels, in comparison to commercially available membranes (for example, Neosepta AMX has a charge density of 4.8 M and Neosepta CMX has a charge density 5.7 $\mathrm{M}$ [39]), have a low number of charged groups per gram of water. We attribute this to the hydrophilic structure of the hydrogels and their capability to hold large amounts of water in their polymeric meshes. This can explain the moderate permselectivity of both hydrogels, as the permselectivity of an ion-selective membrane is related to its charge density $[23,37,38]$. Both anionand cation-exchange hydrogels are therefore not fully selective for the counter ions, which has an adverse effect on the desalination efficiency. However, low salt concentrations lead to a decreased screening of the charged groups present in the hydrogels, thereby promoting charge separation [23]. To demonstrate the successfull functioning of the platform, we therefore performed the desalination ex- 
periments using a salt concentration of maximum $1 \mathrm{mM} \mathrm{NaCl}$. This concentration is two orders of magnitude smaller than the salt concentrations of $0.1 \mathrm{M} / 0.5 \mathrm{M}$, which is commonly used in permselectivity measurements. The resistivity of the hydrogels is larger than that of a typical commercial membrane with an area resistance of $3 \Omega \mathrm{cm}^{2}$ and a membrane thickness of $150 \mu \mathrm{m}$, resulting in a resistivity of $200 \Omega \mathrm{cm}[37,38]$. The relatively high resistivity of our hydrogels is probably related to their low charge density, giving rise to a low concentration of mobile counter ions.

\subsubsection{Desalination - proof of principle experiments}

When filling the microfluidic channels with electrolyte solution and applying a gradually increasing potential $(\Delta V=0-3 \mathrm{~V})$, we observed migration of the fluorescent dye (and thus presumably of the ions) into the hydrogels. A concentration of $5 \mu \mathrm{M}$ of Alexa Fluor 488 Cadaverine was utilised. This negatively charged fluorescent dye is considered to be $p \mathrm{H}$ insensitive and has a low degree of photobleaching [40]. As expected, the AEHs were observed to have a high fluorescence intensity, as they absorb the negatively charged fluorescent dye as a counter-ion to a much greater extent than the CEHs, where the dye is the co-ion.

To test whether the stack of charged hydrogels can be used for desalination purposes, we filled all microchannels with a diluted salt solution $(0.1 \mathrm{mM} \mathrm{NaCl})$ and did not impose any flow through the microchannels. Upon application of a potential difference, depletion zones of the fluorescent dye formed adjacent to the hydrogels in the expected microchannels (which are channels 1, 3 and 5 as shown in Fig. 8.1(b)), while the average fluorescence intensity in the enriched microchannels increased. Snapshots of this observation are shown in the appendix/Fig. 8.13. These observations indicate that the hydrogels are charge-selective and that they can be used for desalination.

IV-sweeps were performed to determine the characteristic underlimiting, limiting and overlimiting current regimes, which are well known for ion-selective membrane systems $[2,4,8,9]$. These measurements were performed for different $\mathrm{NaCl}$ concentrations $(0.01,0.1$ and $1 \mathrm{mM})$ and flow rates $(2,3,5$ and $10 \mu \mathrm{L} / \mathrm{min})$. Relatively low salt concentrations were used to avoid the possible adverse effects occurring at high current densities (e.g. extensive electrode reactions or gas formation). The applied voltage was ramped up with steps of $1 \mathrm{~V}$, with a speed of 10 seconds per voltage step. Fig. 8.4 shows the IV-curves for different salt concentrations. We observed higher currents for higher salt concentrations, indicating that the current in the Ohmic regime is proportional to the ion concentration in the liquid. The maximum current, which is reached at the highest potential difference of $\Delta V=50 \mathrm{~V}$ in the overlimiting current regime, does not precisely scale one to one with the salt concentration.

Normalisation of the current $I$ by the maximum current $I_{\max }$ of the correspond- 
(a) Absolute current $I$ versus $\Delta V$

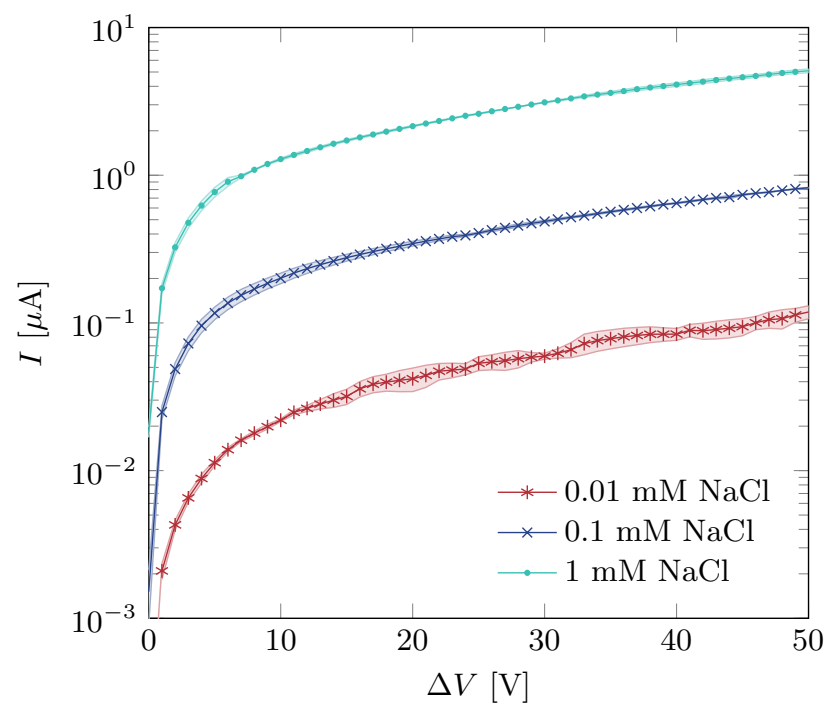

(b) Normalized current $I / I_{\max }$ versus $\Delta V$

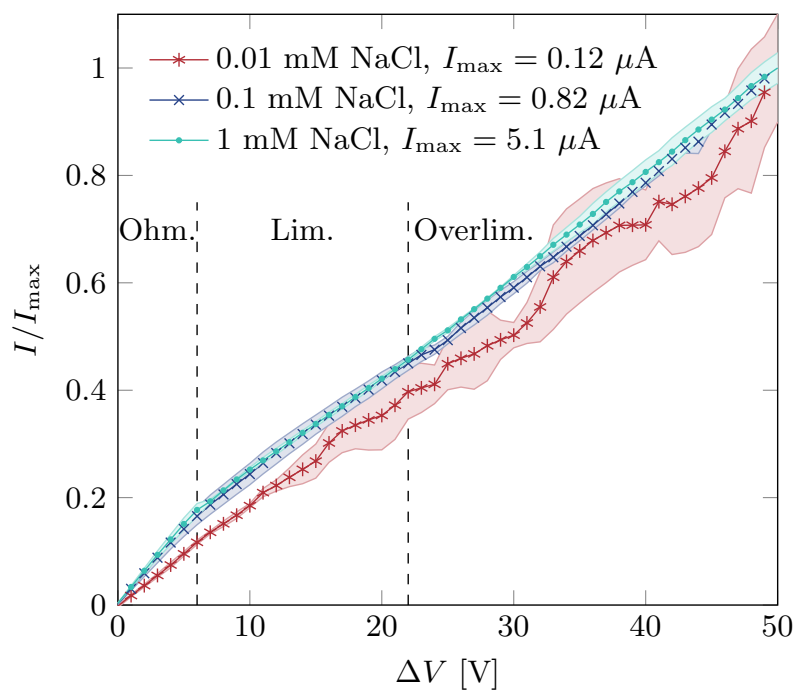

Figure 8.4 - IV characteristics for different salt concentrations at flow rates of $3 \mu \mathrm{L} / \mathrm{min}$. In $(a)$, the absolute current $I$ is plotted as a function of the potential difference $\Delta V$. The measured currents strongly depend on the salt concentration and scale accordingly. In $(b)$, the currents are normalised by the maximum current of the corresponding experiment. The graphs, in particular those for 0.1 and $1 \mathrm{mM}$, show that around a potential difference of approximately $\Delta V=6 \mathrm{~V}$ and $\Delta V=22 \mathrm{~V}$, the slope changes. This indicates where the boundaries between the Ohmic, limiting and overlimiting current regimes are approximately located. 
(a) $0.1 \mathrm{mM} \mathrm{NaCl}, 3 \mu \mathrm{L} / \mathrm{min}$ flow at $\Delta V=20 \mathrm{~V}$
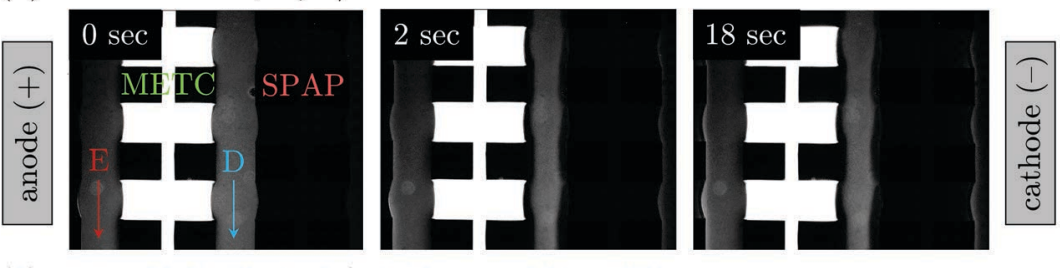

(b) $0.1 \mathrm{mM} \mathrm{NaCl}, 3 \mu \mathrm{L} / \mathrm{min}$ flow at $\Delta V=30 \mathrm{~V}$
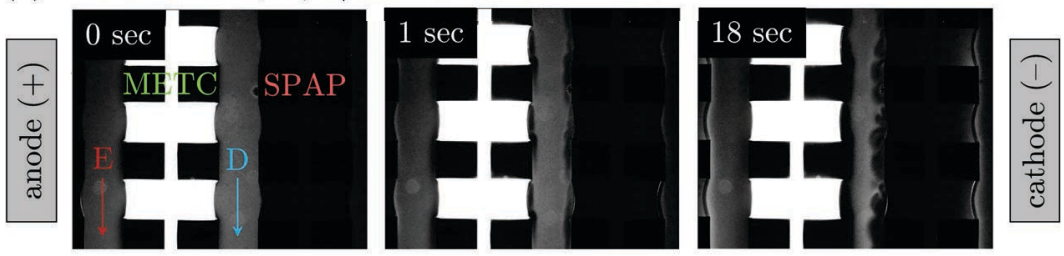

Figure 8.5 - Fluorescence images showing the formation of ion depleted and enriched zones in the depleted (D) and enriched (E) microchannels for two constant potential measurements. A $0.1 \mathrm{mM} \mathrm{NaCl}$ solution containing $5 \mu \mathrm{M}$ Alexa Fluor 488 Cadaverine (the fluorescent dye) was flushed through the microchip at $3 \mu \mathrm{L} / \mathrm{min}$. The experiments were performed at a constant potential difference of (a) $\Delta V=20 \mathrm{~V}$ and (b) $\Delta V=30 \mathrm{~V}$. The bright coloured hydrogel is the anion-exchange hydrogel (METC) and the dark coloured one is the cation-exchange hydrogel (SPAP).

ing experiment indicates that for all concentrations the transport behaviour is similar. At a potential difference of approximately $\Delta V=6 \mathrm{~V}$, ion transport transitions from the Ohmic to the limiting current regime. At a potential difference of roughly $\Delta V=22 \mathrm{~V}$, the overlimiting current regime starts. Calculation of the derivative $\mathrm{d} I / \mathrm{d} \Delta V$ shows that at these points the slope of the $I(\Delta V)$-curve changes, indicating a change in the transport characteristics. The transition points are in particular apparent from the curves for 0.1 and $1 \mathrm{mM} \mathrm{NaCl}$. The fluctuations in current decreased with increasing salt concentration. Different flow rates did not lead to significant differences in the measured currents. This can possibly be attributed to incomplete depletion of ions in the diluted streams, implying that there are still ions available for charge transport through the hydrogels for all flow rates.

For elevated voltages $(\Delta V>10 \mathrm{~V})$ we observed the development of ion depletion zones in the depleted microchannels. On the other hand, a local increase in fluorescence intensity was observed in the enriched microchannels, indicating the existence of locally higher salt concentrations. As shown in Fig. 8.5(a), the depletion zones were observed to grow in size with increasing applied voltage and distance from the inlet. Fig. 8.5(b) shows that for $\Delta V>25 \mathrm{~V}$ vortices were formed in the depletion zones. Probably because of the discontinuous nature of the hydrogel patches - an alternating pattern of charge-selective hydrogels 
and PDMS - the vortices did not migrate in the direction of the imposed flow as reported by Kwak et al. [15]. At the upstream edge of each hydrogel patch, in the depletion zone, a high fluorescence intensity was observed. This suggests that near those points locally the salt concentration was larger than in the rest of the depletion zones. The mixing of the electrolyte solution next to the PMDS pillars by the vortices could explain this behaviour, as this mixing increases the ion concentration near the PDMS pillar and consequently also near the upstream edge of each hydrogel. Charge transport is thus affected by the heterogeneity of the ion concentration at the hydrogel interface and could even enhance it when compared to a homogeneous ion-selective membrane, as previously discussed in the literature [41].

To quantify the desalination performance of the microchip, we performed experiments in which we applied a constant potential in the range of $0-100 \mathrm{~V}$ or a constant current in the range of 3-7 $\mu \mathrm{A}$, which corresponds to a current density in the range of $12-28 \mathrm{~A} / \mathrm{m}^{2}$ (here, the area is defined as the hydrogel surface area per channel wall). The resulting currents and potentials were measured to confirm that there is charge transport through the hydrogel patches. A $1 \mathrm{mM} \mathrm{NaCl}$ solution (supplemented with $5 \mu \mathrm{M}$ Alexa Fluor 488 Cadaverine) was pumped through the microchannels at a flow rate of either 3 or $5 \mu \mathrm{L} / \mathrm{min}$. The conductivities of the solutions collected at the channel outlets were measured, and the salt concentration were calculated accordingly. As expected, the effluent streams from the microchannels were either salt enriched or depleted in an alternating pattern.

In Fig. 8.6( $a)$ the measured salt concentrations are shown for constant potential experiments for two different flow rates ( 3 and $5 \mu \mathrm{L} / \mathrm{min}$ ). When the flow rate was increased, the residence time and therefore the charge separation decreased. Only the outlet streams of the microchannels 2 to 5 were taken into account. In the outer two microchannels 1 and 6, containing the electrodes and both facing only an anion-exchange hydrogel (see Fig. 8.1), the solution is not necessarily either enriched or depleted because of ions generated by electrode reactions.

In Fig. 8.7 $(a)$, the current $I$ is plotted as a function of time $t$ for a fixed potential difference of $\Delta V=70 \mathrm{~V}$ and a flow rate of $3 \mu \mathrm{L} / \mathrm{min}$. This data corresponds to the results shown for $3 \mu \mathrm{L} / \mathrm{min}$ in Fig. 8.6(a). Fig. 8.7(a) demonstrates that the average current during this experiment was $7.7 \mu \mathrm{A}\left(31.3 \mathrm{~A} / \mathrm{m}^{2}\right)$ at steady state. Based on this current, the total transport was calculated to be $4.8 \times 10^{13}$ ions per second, while $6.0 \times 10^{13}$ ions entered each channel per second. Based on this calculation, we expect a desalination degree of $80 \%$. This is twice as high as the measured value of $40 \%$ for $3 \mu \mathrm{L} / \mathrm{min}$, as Fig. $8.1(a)$ shows. The deviation between the measured and expected desalination degree could be attributed to the low permselectivity of the hydrogels, which leads to non-selective ion transport in addition to selective ion transport.

In Fig. 8.6(b), the salt concentrations of the outlet streams are shown for three 
(a) Outlet concentrations at $\Delta V=70 \mathrm{~V}$ for $1 \mathrm{mM} \mathrm{NaCl}$

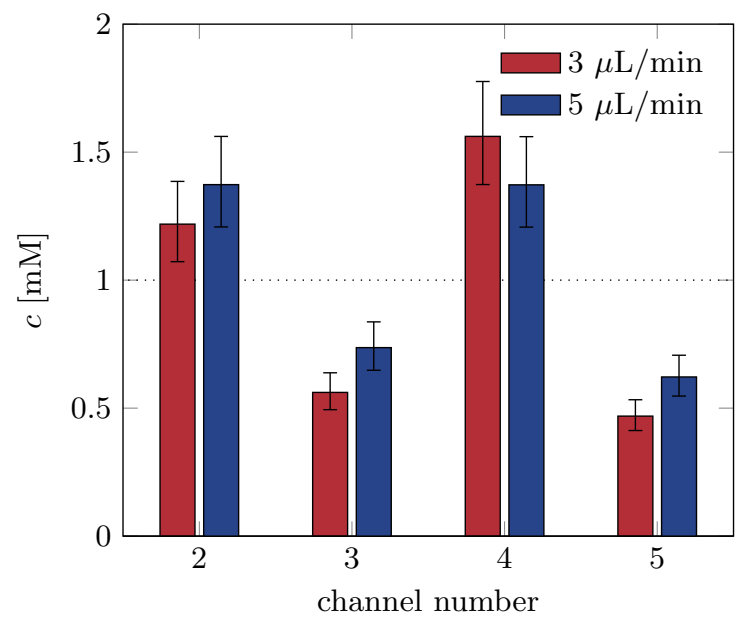

(b) Outlet concentrations at $3 \mu \mathrm{L} / \mathrm{min}$ for $1 \mathrm{mM} \mathrm{NaCl}$



Figure 8.6 - $\mathrm{NaCl}$ concentrations of the solutions collected at the channel outlets for $(a)$ constant potential measurements $(\Delta V=70 \mathrm{~V})$ for different flow rates $(3$ and 5 $\mu \mathrm{L} / \mathrm{min})$, and for $(b)$ constant current measurements with different currents $(3,5$ and 7 $\mu \mathrm{A}$, corresponding to current densities of $12.2,20.3$ and $28.4 \mathrm{~A} / \mathrm{m}^{2}$ ) for a fixed flow rate (3 $\mu \mathrm{L} / \mathrm{min}$ ). The error bars indicate the $95 \%$ prediction bounds.

different applied currents, i.e. for 3, 5, and $7 \mu \mathrm{A}\left(12.2,20.3\right.$ and $\left.28.4 \mathrm{~A} / \mathrm{m}^{2}\right)$. The data shows that the outlet concentrations did not change when increasing the current $I$. Because the transport rate of charge carriers is proportional to the current, we would expect to see a difference in the degree of desalination. For all three currents, however, $75 \%$ of the initially present salt was removed in the 
(a) $1 \mathrm{mM} \mathrm{NaCl}, 3 \mu \mathrm{L} / \mathrm{min}, \Delta V=70 \mathrm{~V}$

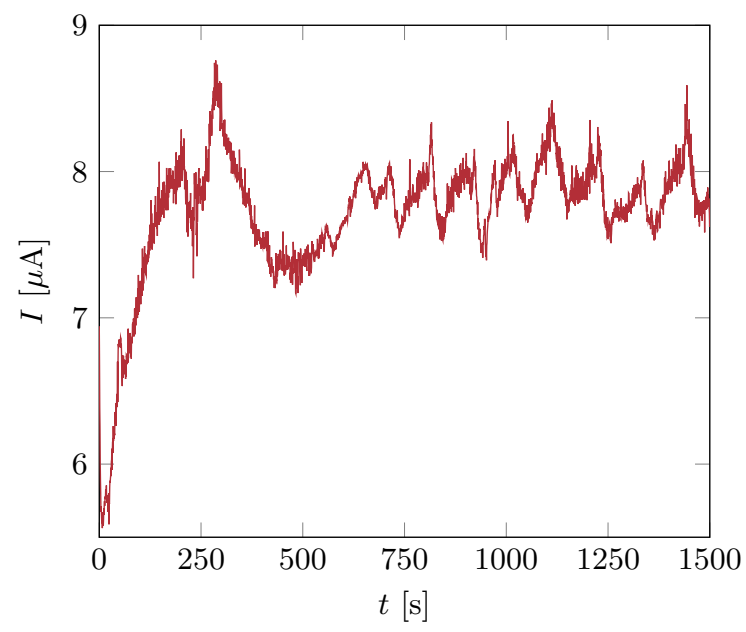

(b) $1 \mathrm{mM} \mathrm{NaCl}, 3 \mu \mathrm{L} / \mathrm{min}$ flow at $\Delta V=70 \mathrm{~V}$
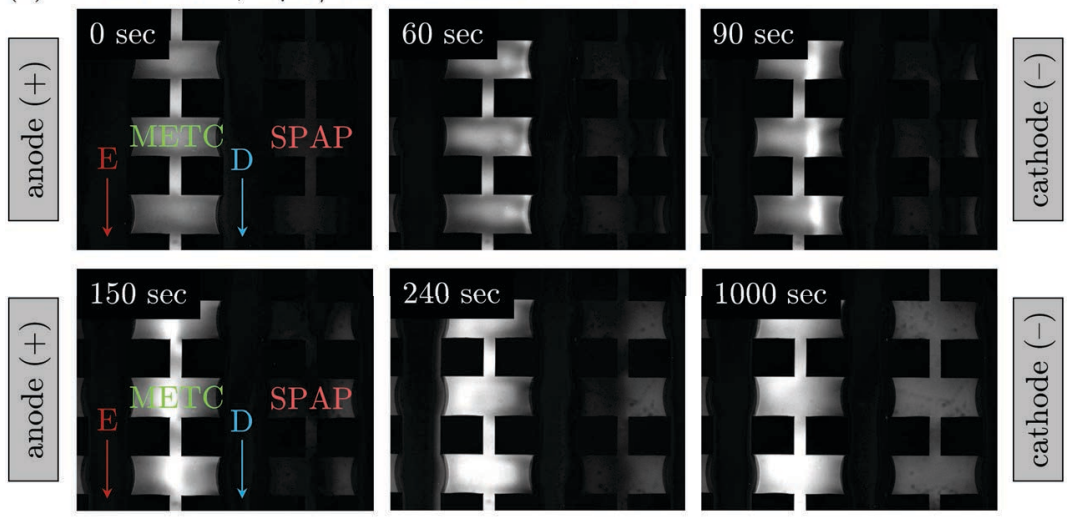

Figure 8.7 - The design of the microfluidic electrodialysis platform allows visualisation of ion transport in the hydrogels between the salt enriched (E) and depleted (D) channels. In $(a)$ the current $I$ is plotted as function of time $t$ for a fixed potential difference of $\Delta V=70 \mathrm{~V}$ and flow rate of $3 \mu \mathrm{L} / \mathrm{min}$. Before reaching steady state, the change in current can be related to transport in the hydrogel, as indicated by the $(b)$ fluorescence images of the dye in the hydrogels $(1 \mathrm{mM} \mathrm{NaCl}$ solution with $5 \mu \mathrm{M}$ Alexa Fluor 488 Cadaverine). It took about $600 \mathrm{~s}$ before the intensity, and thus presumably the dye and ion concentration, in the hydrogels was constant.

desalinated streams. This suggests that $75 \%$ is the maximum desalination degree that is achievable with these devices. As discussed in the previous paragraph, this can be attributed to the presently low charge density of the hydrogels. At higher currents a lower efficiency is then expected.

A valuable property of our microfluidic electrodialysis platform is that it enables 
real-time visualisation of ion transport through the hydrogels using a fluorescent dye. This helps in obtaining a better understanding of the relevant electrokinetic transport phenomena. As the curve in Fig. 8.7( $a)$ shows, approximately 600 seconds were required before the system reached steady state. We observe that at the very first instance $(t<10 \mathrm{~s})$, the current decreased. This could indicate the build-up of a concentration boundary layer. Subsequently the current was increasing, reaching a (local) maximum at $250 \mathrm{~s}$. Inspection of the fluorescence images for this experiment, given in Fig. 8.7(b), shows that the fluorescence distribution can be related to the charge transport through the hydrogels. Directly after applying a potential difference, a high concentration of negatively charged fluorescent dye was observed near the interface of the anion-exchange METChydrogel with the salt-depleted channel. This cluster of fluorescent dye migrated towards the enriched channel in approximately 250 seconds. Comparison with the current profile in Fig. 8.6(a) shows that at that moment, the current reached a (local) maximum. Subsequently, the fluorescence slowly approached a constant intensity distribution, reaching steady state after approximately 600 s. Note that not only in the positively charged anion-exchange hydrogel (AEH) the fluorescence intensity and thus the dye concentration increased, but also in the negatively charged cation-exchange SPAP-hydrogel $(\mathrm{CEH})$. This indicates that, in line with the measured permselectivity of the bulk polymers, the ion selectively of the hydrogels was not perfect. The dye concentration, however, was clearly larger in the $\mathrm{AEH}$ than in the $\mathrm{CEH}$.

\subsection{Conclusion}

Alternatingly patterned anion- and cation-exchange hydrogels were fabricated in a PDMS microchip using capillary barriers. The chemical composition of the hydrogels was tailored to obtain a suitable balance between the degree of swelling and the charge density. The material properties were characterised, and the ion-exchange hydrogels showed ion-selective permeation, based on their ion exchange capacity, permselectivity and resistivity. We demonstrated that the hydrogels can be used for desalination of water with salt concentrations up to 1 $\mathrm{mM} \mathrm{NaCl}$ at various flow rates $(0-5 \mu \mathrm{L} / \mathrm{min})$, applied potentials $(0-100 \mathrm{~V})$, and current densities $\left(12-28 \mathrm{~A} / \mathrm{m}^{2}\right)$. We obtained alternating dilute and concentrated streams at the channel outlets of the microchips. We measured a desalination degree of $40 \%$ for an inlet concentration of $1 \mathrm{mM} \mathrm{NaCl}$, an applied potential difference of $\Delta V=70 \mathrm{~V}$ and a flow rate of $3 \mu \mathrm{L} / \mathrm{min}$. The maximum desalination degree obtained was $75 \%$.

By utilising a charged fluorescent dye, we could visualise the ion concentration in the electrodialysis device. The fluorescence images revealed that ion-depleted 
boundary layers are formed near the ion-exchange hydrogels when a potential difference $\Delta V$ is applied. When $\Delta V>25 \mathrm{~V}$, we observed the formation of vortices, enhancing convective transport towards the hydrogels in the overlimiting current regime. The desalination platform described here also enables visualisation of charge transport through the hydrogels, which can contribute to a better understanding of the transport phenomena in electrodialysis processes.

The separation efficiency can be enhanced by increasing the total length of the hydrogel-liquid interface, by increasing the residence time of the solution in the microchannels, or by optimising the hydrogel properties like the charge density. This would allow desalination of salt solutions with higher concentrations, without the need to apply large potential differences. Our approach might therefore be promising for the development of processes related to water desalination [42], sample pre-treatment [43] and high-focusing of proteins [44].

\section{References}

[1] H. Strathmann, Electrodialysis, a mature technology with a multitude of new applications, Desalination 264, 268 (2010).

[2] V. V. Nikonenko, N. D. Pismenskaya, E. I. Belova, P. Sistat, P. Huguet, G. Pourcelly, and C. Larchet, Intensive current transfer in membrane systems: modelling, mechanisms and application in electrodialysis, Adv. Colloid Interfac. 160, 101 (2010).

[3] M. Tedesco, H. V. M. Hamelers, and P. M. Biesheuvel, Nernst-Planck transport theory for (reverse) electrodialysis : I . Effect of co-ion transport through the membranes, J. Membr. Sci. 510, 370 (2016).

[4] R. W. Baker, in Membrane technology and applications (John Wiley \& Sons, Chichester, 2004).

[5] M. B. Andersen, M. van Soestbergen, A. Mani, H. Bruus, P. M. Biesheuvel, and M. Z. Bazant, Current-induced membrane discharge, Phys. Rev. Lett. 109, 1 (2012).

[6] I. Rubinstein and B. Zaltzman, Convective diffusive mixing in concentration polarization: from Taylor dispersion to surface convection, J. Fluid Mech. 728, 239 (2013).

[7] F. Maletzki, H.-W. Rösler, and E. Staude, Ion transfer across electrodialysis membranes in the overlimiting current range: stationary voltage current characteristics and current noise power spectra under different conditions of free convection, J. Membr. Sci. 71, 105 (1992).

[8] E. Belashova, N. Melnik, N. Pismenskaya, K. Shevtsova, A. Nebavsky, K. Lebedev, and V. Nikonenko, Overlimiting mass transfer through cation- 
exchange membranes modified by Nafion film and carbon nanotubes, Electrochim. Acta 59, 412 (2012).

[9] V. V. Nikonenko, A. V. Kovalenko, M. K. Urtenov, N. D. Pismenskaya, J. Han, P. Sistat, and G. Pourcelly, Desalination at overlimiting currents: state-of-the-art and perspectives, Desalination 342, 85 (2014).

[10] J. H. Lee, Y.-A. Song, and J. Han, Multiplexed proteomic sample preconcentration device using surface-patterned ion-selective membrane, Lab Chip 8, 596 (2008).

[11] S. J. Kim, S. H. Ko, K. H. Kang, and J. Han, Direct seawater desalination by ion concentration polarization, Nat. Nanotechnol. 5, 297 (2010).

[12] H. Kim, J. Kim, E.-G. Kim, A. J. Heinz, S. Kwon, and H. Chun, Optofluidic in situ maskless lithography of charge selective nanoporous hydrogel for DNA preconcentration, Biomicrofluidics 4, 043014 (2010).

[13] L.-J. Cheng and H.-C. Chang, Microscale $p H$ regulation by splitting water, Biomicrofluidics 5, 046502 (2011).

[14] S. J. Kim, S. H. Ko, R. Kwak, J. D. Posner, K. H. Kang, and J. Han, Multi-vortical flow inducing electrokinetic instability in ion concentration polarization layer, Nanoscale 4, 7406 (2012).

[15] R. Kwak, G. Guan, W. K. Peng, and J. Han, Microscale electrodialysis: concentration profiling and vortex visualization, Desalination 308, 138 (2013).

[16] M. Kim, M. Jia, and T. Kim, Ion concentration polarization in a single and open microchannel induced by a surface-patterned perm-selective film, Analyst 138, 1370 (2013).

[17] T. A. Zangle, A. Mani, and J. G. Santiago, Theory and experiments of concentration polarization and ion focusing at microchannel and nanochannel interfaces, Chem. Soc. Rev. 39, 1014 (2010).

[18] S. Park, T. D. Chung, and H. C. Kim, Ion bridges in microfluidic systems, Microfluid. Nanofluid. 6, 315 (2009).

[19] Y. Zhang, N. E. Benes, and R. G. H. Lammertink, Visualization and characterization of interfacial polymerization layer formation, Lab Chip 15, 575 (2015).

[20] D. Hlushkou, R. Dhopeshwarkar, R. M. Crooks, and U. Tallarek, The influence of membrane ion-permselectivity on electrokinetic concentration enrichment in membrane-based preconcentration units, Lab Chip 8, 1153 (2008).

[21] B. Gumuscu, J. G. Bomer, A. van den Berg, and J. C. T. Eijkel, Large scale patterning of hydrogel microarrays using capillary pinning, Lab Chip 15, 664 (2015).

[22] H. Strathmann, Ion-exchange membrane separation processes (Elsevier, Amsterdam, 2004).

[23] T. Sata, Ion exchange membranes: preparation, characterization, modification and application (Royal Society of Chemistry, Cambridge, 2004). 
[24] CRC handbook of chemistry and physics, 76th ed., edited by D. R. Lide (CRC Press, Boca Raton, 1995).

[25] B. Gumuscu, J. G. Bomer, A. van den Berg, and J. C. T. Eijkel, Photopatterning of hydrogel microarrays in closed microchips, Biomacromolecules $\mathbf{1 6}$, 3802 (2015).

[26] W. Zhao, P. Fonsny, P. FitzGerald, G. G. Warr, and S. Perrier, Unexpected behavior of polydimethylsiloxane/poly(2-(dimethylamino)ethyl acrylate) (charged) amphiphilic block copolymers in aqueous solution, Polym. Chem. 4, 2140 (2013).

[27] M. Balastre, S. J. Kostka, and M. McMillan, U.S. patent application no. $14 / 527,812,2015$.

[28] V. V. Khutoryanskiy, Z. S. Nurkeeva, G. A. Mun, A. D. Sergaziyev, Z. Ryskalieva, and J. M. Rosiak, Polyelectrolyte complexes of soluble poly-2[(methacryloyloxy)ethyl]trimethylammonium chloride and its hydrogels with poly(acrylic acid), Eur. Polym. J. 39, 761 (2003).

[29] S. Scognamillo, V. Alzari, D. Nuvoli, J. Illescas, S. Marceddu, and A. Mariani, Thermoresponsive super water absorbent hydrogels prepared by frontal polymerization of $\mathrm{N}$-isopropyl acrylamide and 3-sulfopropyl acrylate potassium salt, J. Polym. Sci. A Polym. Chem. 49, 1228 (2011).

[30] P. M. Biesheuvel, A. P. van Loon, R. Raangs, H. Verweij, and C. Dirksen, A prototype osmotic tensiometer with polymeric gel grains, Eur. J. Soil Sci. 51, 355 (2000).

[31] S. Durmaz and O. Okay, Acrylamide/2-acrylamido-2-methylpropane sulfonic acid sodium salt-based hydrogels: synthesis and characterization, Polymer $\mathbf{4 1}$, 3693 (2000).

[32] O. Okay, in Hydrogel sensors and actuators: Engineering and technology, edited by G. Gerlach and K.-F. Arndt (Springer-Verlag, Berlin, Heidelberg, 2010), pp. 1-14.

[33] E. Pretsch, P. Bühlmann, and M. Badertscher, Structure determination of organic compounds (Springer-Verlag, Berlin, 2009).

[34] Y.-L. Ji, Q.-F. An, Q. Zhao, W.-D. Sun, K.-R. Lee, H.-L. Chen, and C.-J. Gao, Novel composite nanofiltration membranes containing zwitterions with high permeate flux and improved anti-fouling performance, J. Membr. Sci. 390-391, 243 (2012).

[35] G. Beamson and D. Briggs, High resolution XPS of organic polymers: the Scienta ESCA300 database (John Wiley \& Sons, Chicester, 1992).

[36] P. Cardiano, P. G. Mineo, F. Neri, S. Lo Schiavo, and P. Piraino, A new application of ionic liquids: hydrophobic properties of tetraalkylammoniumbased poly(ionic liquid)s, J. Mater. Chem. 18, 1253 (2008).

[37] T. Xu, Ion exchange membranes: state of their development and perspective, J. Membr. Sci. 263, 1 (2005). 
[38] P. Długołecki, K. Nymijer, S. Metz, and M. Wessling, Current status of ion exchange membranes for power generation from salinity gradients, J. Membr. Sci. 319, 214 (2008).

[39] A. H. Galama, J. W. Post, M. A. Cohen Stuart, and P. M. Biesheuvel, Validity of the Boltzmann equation to describe Donnan equilibrium at the membrane-solution interface, J. Membr. Sci. 442, 131 (2013).

[40] N. Panchuk-Voloshina, R. P. Haugland, J. Bishop-Stewart, M. K. Bhalgat, P. J. Millard, F. Mao, W.-Y. Leung, and R. P. Haugland, Alexa dyes, a series of new fluorescent dyes that yield exceptionally bright, photostable conjugates, J. Histochem. Cytochem. 47, 1179 (1999).

[41] S. M. Davidson, M. Wessling, and A. Mani, On the dynamical regimes of pattern-accelerated electroconvection, Sci. Rep. 6, 22505 (2016).

[42] K. N. Knust, D. Hlushkou, R. K. Anand, U. Tallarek, and R. M. Crooks, Electrochemically mediated seawater desalination, Angew. Chem. Int. Edit. 52, 8107 (2013).

[43] P. Kubáň, A. Šlampová, and P. Boček, Electric field-enhanced transport across phase boundaries and membranes and its potential use in sample pretreatment for bioanalysis, Electrophoresis 31, 768 (2010).

[44] R. S. Foote, J. Khandurina, S. C. Jacobson, and J. M. Ramsey, Preconcentration of proteins on microfluidic devices using porous silica membranes, Anal. Chem. 77, 57 (2005). 


\section{Appendix}

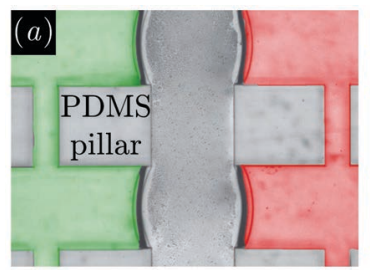

Before DI water injection

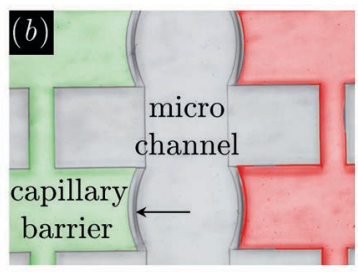

$1.5 \mathrm{~h}$ after injection

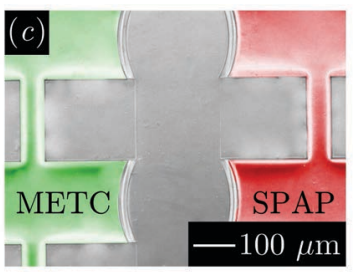

$16 \mathrm{~h}$ after injection

Figure 8.8 - Phase contrast microscopy images of SPAP (red) and METC (green) hydrogels $(a)$ after fabrication, prior to injection of deionised water into the microchannels, $(b)$ after 1.5 hours in water and $(c)$ after 16 hours in water. The hydrogels are artificially coloured based on grey scale differences.

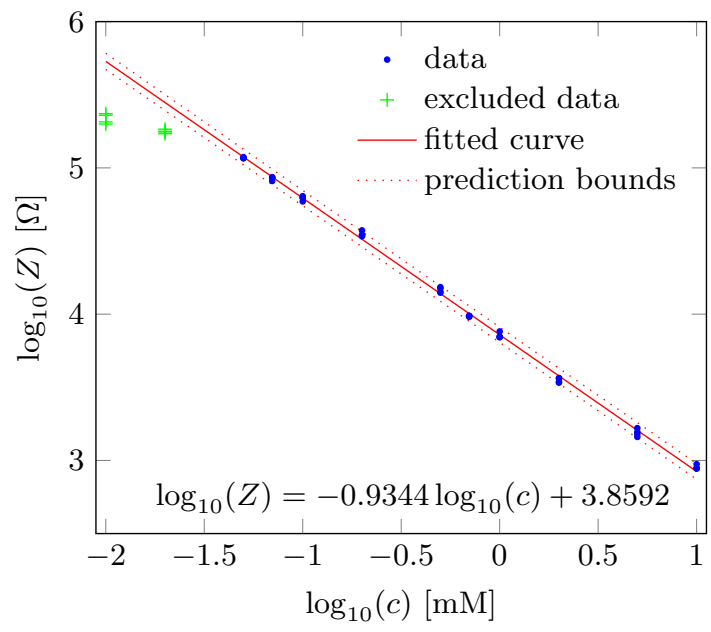

Figure 8.9 - Calibration curve of the impedance $Z$ as a function of the $\mathrm{NaCl}$ concentration $c$. The fitting is performed on a log-log scale to ensure that the experimental data is normally distributed around the calibration curve. The data points for 0.01 and $0.02 \mathrm{mM}$ are omitted, as they clearly fall outside the linear range. This improves the reliability of the calibration curve. The minimum concentration we measured also was always larger than $0.1 \mathrm{mM}$. The dashed lines form the $95 \%$ prediction bounds. As the calibration line gives $\log _{10}(Z)$ as function of $\log _{10}(c)$, on an absolute scale the size of the error depends on the concentration, and the errors are not symmetric around the determined concentration. 
(a) Bis Acrylamide

(b) PA + METC<smiles>C=CC(=O)NCNC(=O)C=C</smiles>

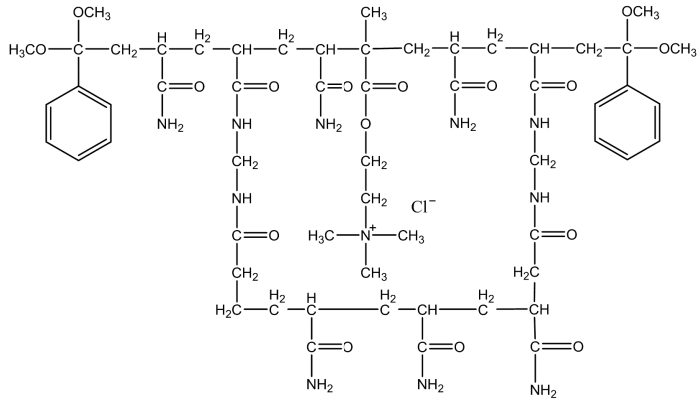

(c) PA + SPAP<smiles>O=C(c1ccccc1)C(O)(O)c1ccccc1</smiles>

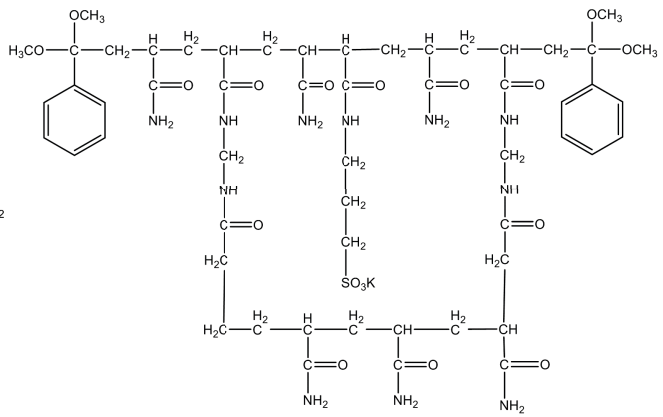

Figure 8.10 - Molecular structures of $(a)$ the various monomers, $(b)$ METC + polyacrylamide, the anion-exchange hydrogel, and $(c)$ SPAP + polyacrylamide, the cationexchange hydrogel.


Figure 8.11 - SEM images of the hydrogels: $(a)$ polyacrylamide, $(b)$ METC + polyacrylamide and $(c)$ SPAP + polyacrylamide. 


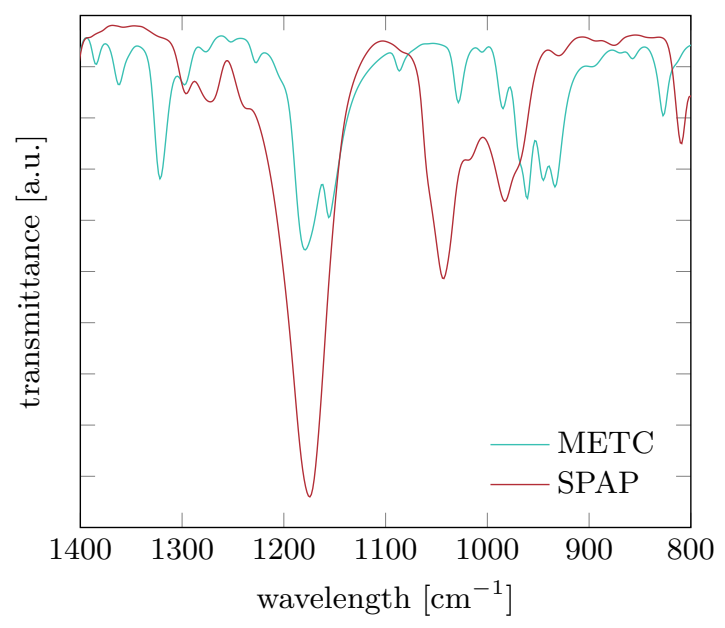

Figure 8.12 - FTIR spectra of the METC and SPAP monomers. For METC, there is a peak at $1178 \mathrm{~cm}^{-1}$ for $\mathrm{C}-\mathrm{N}$ stretching. For SPAP, the peaks at 982 and $1043 \mathrm{~cm}^{-1}$ denote the presence of $\mathrm{R}-\mathrm{SO}_{3}$-groups .

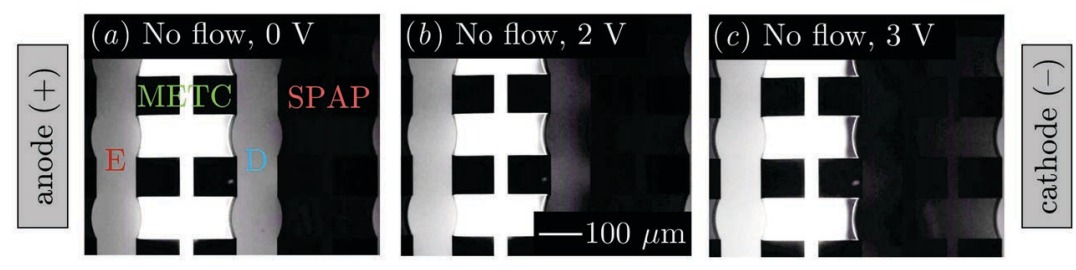

Figure 8.13 - Fluorescence images showing ion concentration polarisation in the microchip. A $1 \mathrm{mM} \mathrm{NaCl}$ solution with $5 \mu \mathrm{M}$ of NaAlexa Fluor 488 Cadaverine (the fluorescent dye) was injected into the microchip and an IV-sweep up to $3 \mathrm{~V}$ was applied. The experiments were performed under an increasing electric field without liquid flow. 




\section{CHAPTER 9}

\section{Conclusions and outlook}

\subsection{Conclusions}

This dissertation describes various studies on the transport of momentum, mass, heat and charge near slippery and/or heterogeneous surfaces. The aim of this work is to extend the current knowledge on transport near such surfaces and to examine how wall slip and/or surface heterogeneity can be employed to enhance interfacial transport.

Most of the research described in this thesis was focused on transport near superhydrophobic substrates. These surfaces are slippery, because they consist of a pattern of no-slip solid walls and no-shear gas bubbles over which a fluid can flow. The amount of effective wall slip in flow over a superhydrophobic bubble mattress can be manipulated by active control of the gas-liquid interface curvature. This can be used to minimise hydrodynamic friction (chapter 2).

Superhydrophobic surfaces are also heterogeneous, giving rise to different hydrodynamic boundary conditions and consequently also to local variations in the flow field. This may lead to some surprising results. Slippage over a bubble mattress is maximised when it is slightly bumpy (chapter 3). In that case the viscous dissipation in the bubble corners, where the no-slip channel wall and the no-shear bubble surfaces meet each other, is minimum. We also found that, in comparison with Newtonian fluids, wall slip is significantly larger for inelastic non-Newtonian liquids when operating the system in the shear-thinning regime (chapter 4). Since complex fluids are encountered frequently, also in the field of (bio)microfluidics, this may offer some potential to improve transport in such non-Newtonian systems.

Because of slippage, convective transport near the interface increases. This can enhance interfacial transport, even in the case of heterogeneously slippery surfaces (chapter 5). As a more generic approach to investigate the relationship between wall slip and heat/mass transfer, we extended the Graetz-Nusselt problem to flows over slippery surfaces (chapter 6 and 7 ). The solutions to this classical forced-convection problem are still widely used in the field of science and engineering. For homogeneous slip flow, an analytical solution could be derived for the Nusselt/Sherwood number in the developing regime that connects and 
actually also encompasses the classical solutions (chapter 6 ). It shows that wall slip can significantly increase interfacial transport when the slip length is of the order of the characteristic size of the system. Wall slip also increases heat/mass transfer in the developed regime in which the transfer rate is constant.

Whereas the solution to the Graetz-Nusselt problem for homogeneous slip flow is valid for both heat and mass transfer, this is not the case for transport near heterogeneously slippery superhydrophobic surfaces (chapter 7). In the case of thermal transport, the no-slip wall has a constant wall temperature and the slippery gas-liquid interface can be treated as adiabatic. For mass transfer, on the other hand, the no-shear gas-liquid interface has a constant wall concentration and the solid wall is impermeable. As only in the case of mass transfer the locations of hydrodynamic slip and mass exchange coincide, mass transfer is more efficient than thermal transport.

These studies show that the local properties of a flow field matter. Small differences in the boundary conditions can therefore lead to a very distinct

performance. Besides the location, also the size of the slip regions is important. When these regions become very small compared to the characteristic size of the system, effective wall slip vanishes and heat/mass transfer near the wall is dominated by conduction/diffusion (chapter 5). In that case, the surface can be treated as non-slippery and as having a uniform temperature/concentration.

Finally, surface heterogeneity also influences ion transport across charge-selective interfaces. We fabricated a microfluidic electrodialysis platform containing a stack of anion- and cation-exchange hydrogels (chapter 8 ). The device allows visualisation of ion transport inside the hydrogels, which can be coupled to the transport phenomena near the hydrogel-water interfaces and in the bulk. This platform can therefore help to investigate and to understand the physical phenomena related to charge transport in more detail.

\subsection{Outlook}

\subsubsection{Enhanced transport}

An important condition for employing wall slip to enhance interfacial transport is that the typical length scale of the system needs to match with the effective slip length. In that sense, wall slip opens up a wide range of possibilities for nano- and microfluidic systems [1-5]. Slippery surfaces can also be of interest in large-scale applications in which small scale conduits play an important role. Examples include membrane systems consisting of hollow fibres [6], multiphase monolith reactors [7] and porous media [8].

Consider, for instance, a hollow fibre, which is structured at the inside and/or the outside $[9,10]$. Two examples are shown in Fig. 9.1. When the surface 

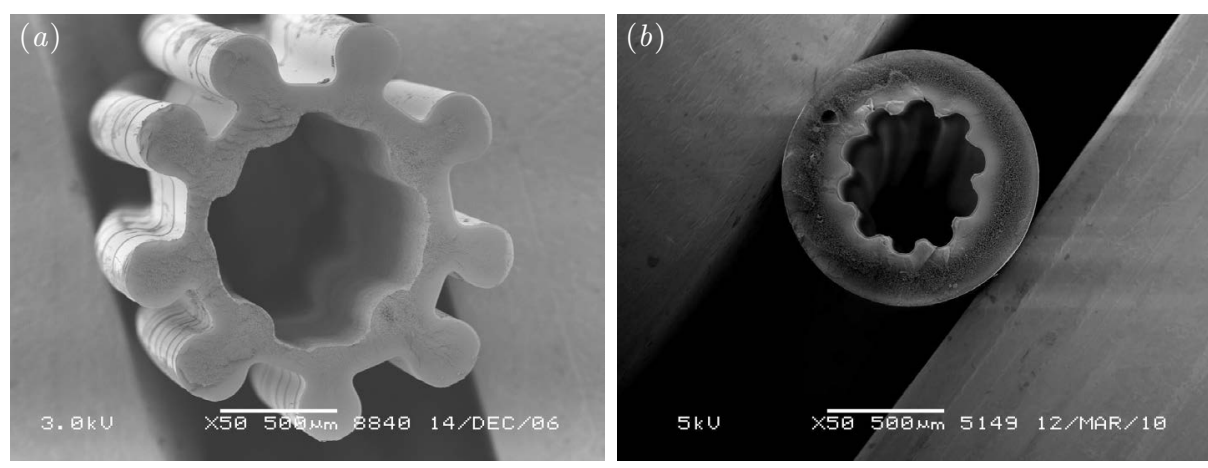

Figure 9.1 - Examples of hollow fibre membranes that are structured from $(a)$ the outside [9] or (b) the inside [10], fabricated from a polyethersulfone/polyvinylpyrrolidone blend.

structures are small enough and the membrane material itself is hydrophobic and therefore gas-filled, a superhydrophobic surface can be created. The way these membranes are produced entails that inside the fibres the flow is parallel to the flow direction. Such structured hollow fibre membranes are therefore potentially highly slippery, which may benefit interfacial transport.

Producing surface structures that are small enough to ensure the formation of stable gas-liquid menisci remains challenging though [9, 10]. A possible way to overcome this is by using other production techniques, such as three-dimensional prototyping of membranes [11]. Besides topologically structured, it is worth to study whether such structured hollow fibres can also be made chemically heterogeneous by functionalisation of the surface [12] or by (locally) adding catalytic [13] or charged materials [14]. This could induce surface-driven flows, discussed in Sec. 9.2.3, which can promote convective transport at the microscopic scale near the interface between the fibre and the liquid.

\subsubsection{Visco-elasticity and wall slip}

When flowing over a heterogeneous substrate, the varying surface conditions can give rise to characteristic time scales, even when the flow is stationary. For a bubble mattress, for instance, this would correspond to the time required to flow over one no-slip/no-shear bubble unit. In the case of visco-elastic fluids, such as polymer solutions, the typical time scale $t$ could potentially match the relaxation time $\tau$ of the fluid. Depending on the relative time scale, as expressed by the Deborah number $D e=\tau / t$, the response of a visco-elastic fluid is either predominantly elastic $(D e<1)$ or principally viscous $(D e>1)$. In chapter 4 we treated the aqueous xanthan gum solutions as inelastic and shear thinning. Fig. 9.2 


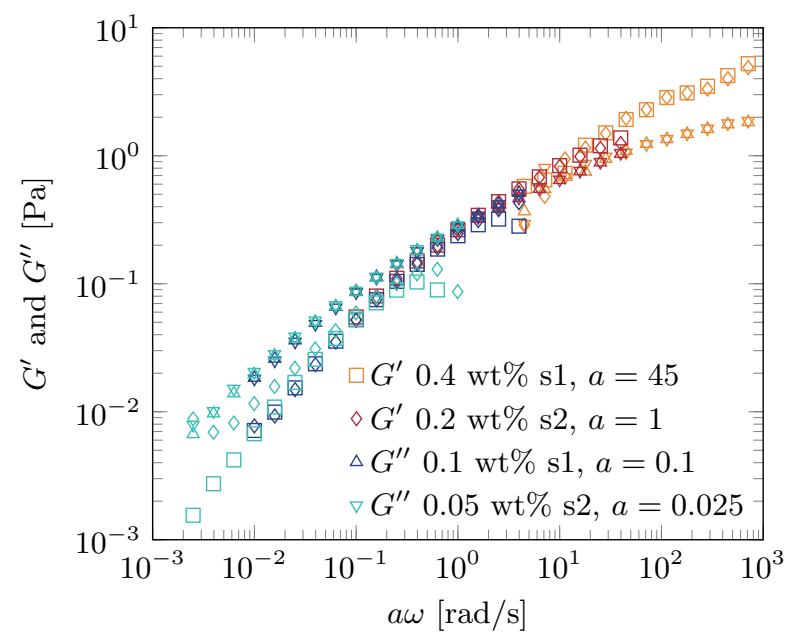

Figure 9.2 - Dynamic measurement data for multiple aqueous xanthan gum solutions, time-concentration shifted to the reference concentration of $0.2 \mathrm{wt} \%$. The square and diamond symbols in the figure represent the storage modulus $G^{\prime}$. The viscous modulus $G^{\prime \prime}$ is given by the upward and downward pointing triangles. The colours denote different xanthan gum concentrations. The shift factor $a=\tau_{0} / \tau$ is the ratio of the relaxation time of the reference concentration to that of the concentration under consideration. A strain sweep performed at a frequency of $f=1 \mathrm{~Hz}$ showed that the visco-elastic regime is linear for strains up to $40 \%$.

illustrates that these solutions actually are visco-elastic, with a characteristic time scale of $\tau \sim 1 \mathrm{~s}$ for a $0.2 \mathrm{wt} \%$ solution. However, since both the elastic and viscous modulus are very small, i.e. $G^{\prime}, G^{\prime \prime}<10 \mathrm{~Pa}$, xanthan gum can be treated as (nearly) inelastic [15].

Microfluidic platforms are highly suitable to study and employ visco-elastic fluids, as high shear rates $\gamma$ can be reached [16]. Surface heterogeneity leads to local variations in this shear rate, and consequently the rate of deformation also varies spatially. This can give rise to high Weissenberg numbers $W i=\tau \gamma$, denoting the product of relaxation time and fluid deformation rate (note that the time scale $t$ does not necessarily equal the inverse of the deformation rate $1 / \gamma$, and consequently also the Deborah and Weissenberg numbers can be different [17]). Elastic effects in microscale systems can therefore be significant.

Because the flow of visco-elastic fluids can lead to various phenomena not observed for Newtonian fluids, visco-elasticity can been exploited in a number of different applications in which non-Newtonian fluids are encountered. It has been predicted that by applying an oscillating driving force the transverse velocity components in microchannels can be increased, which will promote mixing $[18,19]$. Complex fluids can be employed as a working fluid to stabilise flows at 
low Reynolds numbers through highly anisotropic channels: so-called microfluidic rectifiers [20-22]. For the operation of these devices, visco-elasticity provides the required non-linearity. Also obstacles in the flow can create elastic instabilities $[23,24]$, which affects the flow profile around the object.

It is unknown how effective wall slip is affected by visco-elastic behaviour. For slippery superhydrophobic surfaces, the heterogeneous boundary induces local variations in shear that can be considerable. Besides that, the entrapped gas bubbles can protrude in and act as an obstacle to the flow. This could lead to various interesting effects in the fluid flow, such as instabilities and reduced friction. The combination of non-linear fluid properties and slippery (and protruding) bubbles may also have a positive effect on interfacial transport.

\subsubsection{Surface-driven flows}

Both smooth and superhydrophobic surfaces offer great potential for systems with interfacially driven transport, even though intrinsic slip lengths for smooth surfaces are only tens of nanometres long $[25,26]$. Consider for instance electroosmotic flow (EOF), in which an electric field $E$ generates a volume force in the electrical double layer (EDL) near a surface with a zeta potential $\zeta$ at the slipping plane. In this double layer, the ion concentration is unbalanced. The resulting net Coulomb force acting in this layer induces motion of the fluid near the surface. Since the characteristic thickness of the electrical double layer is characterised by the Debye length $\lambda_{D}$, which is approximately $10 \mathrm{~nm}$ for a $1 \mathrm{mM}$ monovalent salt solution [27], all velocity gradients are located very close to the wall. Generally the EDL is very thin and much smaller than the characteristic channel height $H$. As a consequence, essentially a plug flow with a velocity $U_{\text {eof }}$ is generated. In case of a slippery surface, the electro-osmotic velocity profile is written as $[27,28]$

$$
U_{\text {eof }}=-\frac{\epsilon_{m} \zeta E}{\mu}\left(1+\frac{b}{\lambda_{D}}\right)\left(1-\exp \left(-\frac{y}{\lambda_{D}}\right)\right)
$$

where $\epsilon_{m}$ is the dielectric permittivity of the medium. It immediately follows that electro-osmotic flow can be enhanced with a factor $\left(1+b / \lambda_{D}\right)$. This enhancement factor can be significant, as even intrinsic slip lengths can be much larger than the Debye layer, which is usually 1 to $100 \mathrm{~nm}$ thick for watery systems $[1,29,30]$. Using total internal reflection fluorescence (TIRF), and compared to EOF over a hydrophilic glass surface, Bouzigues et al. [31] measured a doubling of the electro-osmotic velocity $U_{\text {eof }}$ for a homogeneously slippery hydrophobised glass surface.

Electro-osmotic flow over superhydrophobic surfaces is more complicated, and it is not until recently that effects of heterogeneous surfaces, which are anisotropic regarding local wall slip as well as surface charge, on EOF were explored. Since 
the velocity gradients for EOF are only present in de Debye layer, the flow over the surface is directly affected when introducing small patterns on the surface which are characterised by different zeta potentials, as theoretical and experimental studies show [32-34]. For surfaces with a pattern of opposite surface charges, locally a flow reversal was observed.

Analytical and numerical studies [28, 35-39] predict what will happen when a pattern of different wall slip is combined with variations in zeta potential. The resulting EOF field is the result of the complex interplay of the relative amount of slip length $b$ to the typical length of the surface pattern $L$. Depending on the ratio of the zeta potentials and the relative size of local $\left(b_{l}\right)$ to effective $\left(b_{f}\right)$ slip length, EOF may be inhibited, enhanced or reversed. For a weakly charged surface containing patches of no-slip (region $1, b_{l}=0$ ) and partial slip (region $2, b_{l}$ ), being in the thin double layer limit where $\lambda_{D} / L \ll 1$, the electro-osmotic velocity is given by [36]

$$
U_{\mathrm{eof}}=-\frac{\epsilon_{m} \zeta_{1} E}{\mu}\left[1+\frac{b_{f}}{b_{l}}\left(\frac{q_{2}}{q_{1}}\left(1+\frac{b_{l}}{\lambda_{D}}\right)-1\right)\right],
$$

where $q_{i}$ is the constant charge density of the surface, which is related to the zeta potential as $\zeta_{i}=q_{i} \lambda_{D} / \epsilon_{m}$.

We can distinguish various situations. In the case of slip regions that are uncharged but perfectly slippery, $U_{\text {eof }}=U_{0}=-\left(\epsilon_{m} \zeta_{1} E\right) / \mu$ and therefore no enhancement is expected. When the slip regions are uncharged but not perfectly slippery, which is not unrealistic, even for superhydrophobic surfaces, EOF is actually suppressed in comparison with a homogeneous no-slip surface with a uniform charge density. Enhancement is possible, however, when the slip regions would carry some charge. When the surface would have a homogeneous charge, i.e. $q_{1}=q_{2}$, Eq. (9.1) holds again. In that case the amplification factor $\left(1+b_{f} / \lambda_{D}\right)$ can be very large, as $b_{f}=\mathcal{O}(\mu \mathrm{m})$.

Electro-osmotic flow over heterogeneously slippery and charged surfaces has not been investigated experimentally yet. Besides increasing momentum transport, such surfaces could also be employing to improve mixing [40]. It is therefore of high interest to provide experimental evidence for the numerically and analytically predicted flow behaviour over such surfaces. This may be challenging experimentally, as it requires quantitative determination of the flow fields, which then have to be correlated with accurate information on local slip lengths and zeta potentials.

In Fig. 9.3, a possible experimental approach is schematically shown. First, a heterogeneous surface needs to be fabricated. This can be a chemically heterogeneous surface with patches of different zeta potentials, with one of those showing (partial) slip. Also superhydrophobic surfaces could be used for this. The flow field is predicted to depend strongly on the surface conditions. The flow field given 
(a) EOF in microfluidic device

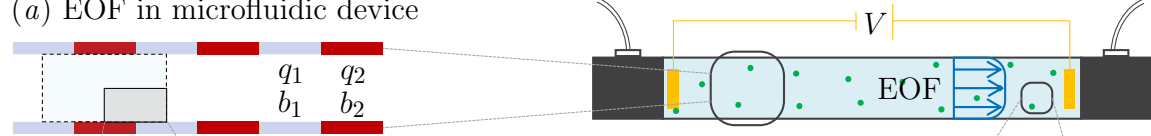

(b) $q_{2} / q_{1}=0.5$

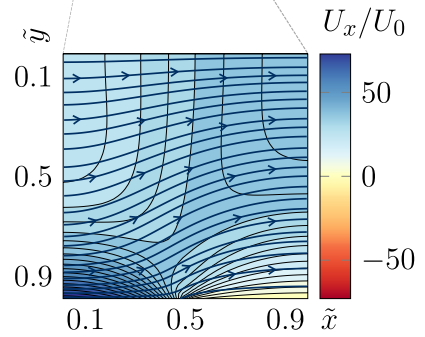

(c) $q_{2} / q_{1}=-0.02$

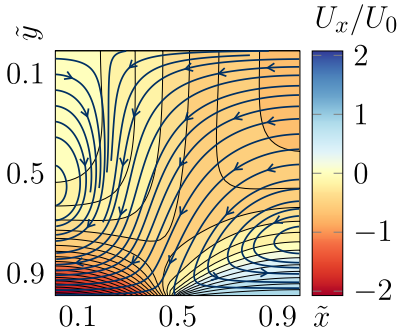

(d) $\mu \mathrm{PIV}$ and TIRF

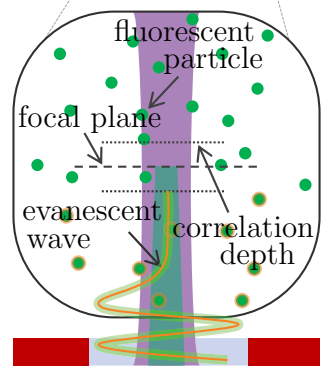

Figure 9.3 - Schematic drawing ( $a$ ) of a microfluidic device allowing electro-osmotic flow over a surface that is heterogeneous regarding both wall slip $b$ and surface charge $q$. In $(b)$ and $(c)$, two examples of the flow field are given for a system with a pattern length $L=40 \mu \mathrm{m}$, a channel height $H=80 \mu \mathrm{m}$, a slip fraction of $\varepsilon=1 / 2$ and with slip lengths of $b_{1}=0$ and $b_{2}=L$. The Debye length is $\lambda_{D}=30 \mathrm{~nm}$, but the ratio of the surface charges varies. These flow profiles, calculated using the solution reported by $\mathrm{Ng}$ and Chu [37], illustrate therefore possible flow profiles over a slippery superhydrophobic surface. A flow reversal is observed in $(c)$ for oppositely charged surface patches. The velocity field can be measured experimentally $(d)$ using micro-partical image velocimetry $(\mu \mathrm{PIV})$ and/or total internal reflection fluorescence (TIRF).

in Fig. 9.3(b), for which the ratio of zeta potentials equals 0.5 [41-45], shows a strong enhancement in flow as $U_{x} \gg U_{0}$. Surfaces can also acquire a negative zeta potential, which could also be induced actively [46, 47]. As Fig. 9.3(c) shows, even when $-q_{2} \ll q_{1}$ already a reversal of the flow over the slippery, charged surface is predicted to occur. The flow fields can be measured experimentally near the surface using total internal reflection fluorescence (TIRF) or in the bulk of the microchannels using micro-particle image velocity $(\mu \mathrm{PIV})$.

These experiments can be extended to other surface driven flows, such as diffusioosmosis and thermo-osmosis where a flow is induced by a solute or temperature gradient near a slippery wall $[25,48]$. Also for these interfacially driven transport phenomena, enhancement factors of $(1+b / L)$ can be obtained:

$$
U=-\frac{1}{\mu} \Gamma L\left(1+\frac{b}{L}\right) \frac{\mathrm{d} O}{\mathrm{~d} x},
$$

with $O$ being a certain observable, like temperature or concentration, $\Gamma$ being a length scale measuring the excess of the observable near the surface, and $L$ being 
the thickness of the interface in which a stress is generated by the observable $O$ [48].

This offers possibilities to employ wall slip near catalytic surfaces containing hydrophilic and hydrophobic patches [49], or near surfaces that contain patches with different catalytic activities [50-52]. By electrochemical reduction of peroxide, which is generating an electric field, platinum/gold particles start to move by reaction-induced auto-electrophoresis [53]. In all these examples, there are concentration gradients very close to the surface. According to Eq. (9.3), slippage could be used in such systems to increase convection very close to the surface by transporting reactants faster to or from the catalytically active sites. This potentially enhances the efficiency of a catalytic process.

\subsubsection{Electrokinetic transport and wall heterogeneity}

Wall heterogeneity can be used to enhance ion transport across charge-selective and permeable surfaces. Spatial variations in, for instance, the dielectric permittivity of the wall lead to local deviations in the electric field, which promotes electroconvection [54]. Systematic experimental studies on this predicted behaviour are limited, however. The fabrication method of the microfluidic electrodialysis platform described in chapter 8 makes it possible to vary the wall geometry relatively easily, which facilitates a systematic study on the relationship between wall geometry and charge transport.

The aim is to fully characterise the transport phenomena that are related to electrodialysis by spatially resolving the concentration of the chloride ions using fluorescence lifetime imaging (FLIM) in combination with, for example, a chloride-sensitive dye. The device allows visualisation of the charge transport inside the transparent hydrogels, which form the permselective barriers between the microchannels. These charge-selective hydrogels enable the desalination of salt water under an electric field. Additionally, dispersion of (preferably) chargeneutral fluorescent particles can provide more detailed information on the flow profile. Of particular interest is the flow pattern near the water-hydrogel interfaces at the onset of the overlimiting current regime. In this way, charge transport in the channels, near the interfaces and in the hydrogels can be coupled to the flow profile in the channels.

\section{References}

[1] H. A. Stone, A. D. Stroock, and A. Ajdari, Engineering flows in small devices: microfluidics toward a lab-on-a-chip, Annu. Rev. Fluid Mech. 36, 381 (2004).

[2] V. Hessel, H. Löwe, and F. Schönfeld, Micromixers - a review on passive and active mixing principles, Chem. Eng. Sci. 60, 2479 (2005). 
[3] K. F. Lam, E. Sorensen, and A. Gavriilidis, Review on gas-liquid separations in microchannel devices, Chem. Eng. Res. Des. 91, 1941 (2013).

[4] E. Y. Kenig, Y. Su, A. Lautenschleger, P. Chasanis, and M. Grünewald, Microseparation of fluid systems: a state-of-the-art review, Sep. Purif. Technol. 120, 245 (2013).

[5] E. K. Sackmann, A. L. Fulton, and D. J. Beebe, The present and future role of microfluidics in biomedical research, Nature 507, 181 (2014).

[6] A. Gabelman and S.-T. Hwang, Hollow fiber membrane contactors, J. Membr. Sci. 159, 61 (1999).

[7] M. T. Kreutzer, F. Kapteijn, J. A. Moulijn, and J. J. Heiszwolf, Multiphase monolith reactors: chemical reaction engineering of segmented flow in microchannels, Chem. Eng. Sci. 60, 5895 (2005).

[8] S. Berg, A. W. Cense, J. P. Hofman, and R. M. M. Smits, Two-phase flow in porous media with slip boundary condition, Tranp. Porous Med. 74, 275 (2008).

[9] P. Z. Çulfaz, E. Rolevink, C. van Rijn, R. G. H. Lammertink, and M. Wessling, Microstructured hollow fibers for ultrafiltration, J. Membrane Sci. 347, 32 (2010).

[10] P. Z. Çulfaz, M. Wessling, and R. G. H. Lammertink, Hollow fiber ultrafiltration membranes with microstructured inner skin, J. Membr. Sci. 369, 221 (2011).

[11] T. Femmer, A. J. C. Kuehne, and M. Wessling, Estimation of the structure dependent performance of 3-D rapid prototyped membranes, Chem. Eng. J. 273, 438 (2015).

[12] S. P. Sun, K. Y. Wang, N. Peng, T. A. Hatton, and T.-S. Chung, Novel polyamide-imide/cellulose acetate dual-layer hollow fiber membranes for nanofiltration, J. Membr. Sci. 363, 232 (2010).

[13] R. Kas, K. K. Hummadi, R. Kortlever, P. de Wit, A. Milbrat, M. W. J. LuitenOlieman, N. E. Benes, M. T. M. Koper, and G. Mul, Three-dimensional porous hollow fibre copper electrodes for efficient and high-rate electrochemical carbon dioxide reduction, Nat. Commun. 7, 10748 (2016).

[14] C. V. Gherasim, T. Luelf, H. Roth, and M. Wessling, Dual-charged hollow fiber membranes for low-pressure nanofiltration based on polyelectrolyte complexes: one-step fabrication with tailored functionalities, ACS Appl. Mater. Interfaces 8, 19145 (2016).

[15] P. E. Arratia, G. A. Voth, and J. P. Gollub, Stretching and mixing of non-Newtonian fluids in time-periodic flows, Phys. Fluids 17, 1 (2005).

[16] M. S. N. Oliveira, M. A. Alves, and F. T. Pinho, Transport and mixing in laminar flows (Wiley-VCH Verlag, Weinheim, 2011), pp. 131-174.

[17] R. J. Poole, The Deborah and Weissenberg numbers, Rheol. Bull. 53, 32 (2012). 
[18] G. G. Pereira, Effect of variable slip boundary conditions on flows of pressure driven non-Newtonian fluids, J. Non-Newt. Fluid Mech. 157, 197 (2009).

[19] S. Dhondi, G. G. Pereira, and S. C. Hendy, Molecular dynamics simulations of polymeric fluids in narrow channels: methods to enhance mixing, Phys. Rev. E 80, 036309 (2009).

[20] A. Groisman, Microfluidic memory and control devices, Science 300, 955 (2003).

[21] A. Groisman and S. R. Quake, A microfluidic rectifier: anisotropic flow resistance at low Reynolds numbers, Phys. Rev. Lett. 92, 094501 (2004).

[22] P. C. Sousa, F. T. Pinho, M. S. N. Oliveira, and M. A. Alves, Efficient microfluidic rectifiers for viscoelastic fluid flow, J. Non-Newt. Fluid Mech. 165, 652 (2010).

[23] V. M. Ribeiro, P. M. Coelho, F. T. Pinho, and M. A. Alves, Viscoelastic fluid flow past a confined cylinder: three-dimensional effects and stability, Chem. Eng. Sci. 111, 364 (2014).

[24] K. P. Nolan, A. Agarwal, S. Lei, and R. Shields, Viscoelastic flow in an obstructed microchannel at high Weissenberg number, Microfluid. Nanofluid. 20, 101 (2016).

[25] L. Bocquet and J.-L. Barrat, Flow boundary conditions from nano- to microscales, Soft Matter 3, 685 (2007).

[26] J. C. T. Eijkel, Liquid slip in micro- and nanofluidics: recent research and its possible implications, Lab Chip 7, 299 (2007).

[27] H. Bruus, Theoretical microfluidics (Oxford University Press, Oxford, 2009).

[28] T. M. Squires, Electrokinetic flows over inhomogeneously slipping surfaces, Phys. Fluids 20, 092105 (2008).

[29] L. Joly, C. Ybert, E. Trizac, and L. Bocquet, Hydrodynamics within the electric double layer on slipping surfaces, Phys. Rev. Lett. 93, 257805 (2004).

[30] L. Joly, C. Ybert, E. Trizac, and L. Bocquet, Liquid friction on charged surfaces: from hydrodynamic slippage to electrokinetics, J. Chem. Phys. 125, 204716 (2006).

[31] C. I. Bouzigues, P. Tabeling, and L. Bocquet, Nanofluidics in the Debye layer at hydrophilic and hydrophobic surfaces, Phys. Rev. Lett. 101, 114503 (2008).

[32] A. Ajdari, Electro-osmosis on inhomogeneously charged surfaces, Phys. Rev. Lett. 75, 755 (1995).

[33] A. E. Herr, J. I. Molho, J. G. Santiago, M. G. Mungal, T. W. Kenny, and M. G. Garguilo, Electroosmotic capillary flow with nonuniform zeta potential, Anal. Chem. 72, 1053 (2000).

[34] A. D. Stroock, M. Weck, D. T. Chiu, W. T. Huck, P. J. Kenis, R. F. Ismagilov, and G. M. Whitesides, Patterning electro-osmotic flow with patterned surface charge, Phys. Rev. Lett. 84, 3314 (2000). 
[35] S. S. Bahga, O. I. Vinogradova, and M. Z. Bazant, Anisotropic electro-osmotic flow over super-hydrophobic surfaces, J. Fluid Mech. 644, 245 (2010).

[36] A. V. Belyaev and O. I. Vinogradova, Electro-osmosis on anisotropic superhydrophobic surfaces, Phys. Rev. Lett. 107, 098301 (2011).

[37] C.-O. Ng and H. C. W. Chu, Electrokinetic flows through a parallel-plate channel with slipping stripes on walls, Phys. Fluids 23, 102002 (2011).

[38] H. C. W. Chu and C.-O. Ng, Electroosmotic flow through a circular tube with slip-stick striped wall, J. Fluids Eng. 134, 111201 (2012).

[39] C. Zhao and C. Yang, Advances in electrokinetics and their applications in micro/nano fluidics, Microfluid. Nanofluid. 13, 179 (2012).

[40] Q. Zhou and C.-O. Ng, Electro-osmotic dispersion in a circular tube with slip-stick striped wall, Fluid Dyn. Res. 47, 015502 (2015).

[41] C. Yang, T. Dabros, D. Li, J. Czarnecki, and J. H. Masliyah, Measurement of the zeta potential of gas bubbles in aqueous solutions by microelectrophoresis method, J. Colloid Interf. Sci. 243, 128 (2001).

[42] A. Sze, D. Erickson, L. Ren, and D. Li, Zeta-potential measurement using the Smoluchowski equation and the slope of the current-time relationship in electroosmotic flow, J. Colloid Interf. Sci. 261, 402 (2003).

[43] B. J. Kirby and E. F. Hasselbrink, Zeta potential of microfluidic substrates: 2. Data for polymers, Electrophoresis 25, 203 (2004).

[44] M. Takahashi, $\zeta$ potential of microbubbles in aqueous solutions: electrical properties of the gas-water interface, J. Phys. Chem. B 109, 21858 (2005).

[45] V. Tandon, S. K. Bhagavatula, W. C. Nelson, and B. J. Kirby, Zeta potential and electroosmotic mobility in microfluidic devices fabricated from hydrophobic polymers: 1. The origins of charge, Electrophoresis 29, 1092 (2008).

[46] C. Steffes, T. Baier, and S. Hardt, Enabling the enhancement of electroosmotic flow over superhydrophobic surfaces by induced charges, Colloids Surf. A $\mathbf{3 7 6}$, 85 (2011).

[47] C. Schönecker and S. Hardt, Electro-osmotic flow along superhydrophobic surfaces with embedded electrodes, Phys. Rev. E 89, 063005 (2014).

[48] A. Ajdari and L. Bocquet, Giant amplification of interfacially driven transport by hydrodynamic slip: diffusio-osmosis and beyond, Phys. Rev. Lett. 96, 186102 (2006).

[49] C. Franch, R. G. H. Lammertink, and L. Lefferts, Partially hydrophobized catalyst particles for aqueous nitrite hydrogenation, Appl. Catal. B 156-157, 166 (2014).

[50] A. Sen, M. Ibele, Y. Hong, and D. Velegol, Chemo and phototactic nano/microbots, Faraday Discuss. 143, 15 (2009).

[51] J. G. Gibbs and Y.-P. Zhao, Autonomously motile catalytic nanomotors by bubble propulsion, Appl. Phys. Lett. 94, 163104 (2009).

[52] C. Zhou, H. Zhang, Z. Li, and W. Wang, Chemistry pumps: a review of 
chemically powered micropumps, Lab Chip 16, 1797 (2016).

[53] J. L. Moran, P. M. Wheat, and J. D. Posner, Locomotion of electrocatalytic nanomotors due to reaction induced charge autoelectrophoresis, Phys. Rev. E 81, 1 (2010).

[54] V. V. Nikonenko, N. D. Pismenskaya, E. I. Belova, P. Sistat, P. Huguet, G. Pourcelly, and C. Larchet, Intensive current transfer in membrane systems: modelling, mechanisms and application in electrodialysis, Adv. Colloid Interf. 160, 101 (2010). 




\section{Acknowledgements}

Having finished my doctoral research and dissertation, I owe many thanks to a number of people without whom I would not have been able to finish this work.

First of all, I would like to express my gratitude to Rob Lammertink as my promotor and daily supervisor. Your ERC starting grant allowed me to start as a PhD student in the Soft matter, Fluidics and Interfaces (SFI) research group. Thank you for all your guidance, knowledge and support. I really felt comfortable with your style of supervision: you were highly supportive and you gave me a lot of freedom. At the same time: you also directed me when needed, without being imperative. You allowed me to develop myself both personally and professionally.

I want to thank Jeff Wood, being my co-promotor, for all the discussions we had. These resulted in a number of very nice collaborations. I admire your vast knowledge on almost every topic. A big part of solving a problem was just talking to you about it. I really enjoyed these scientific sparring sessions!

I would like to extend my gratitude to Amy Tsai as well. During the first part of my $\mathrm{PhD}$, you were involved in many projects. Your view and suggestions improved the work considerably.

Many of the studies I did were collaborations. In particular I want to thank various staff members of the Physics of Fluids research group. I am grateful to Detlef Lohse for the cooperation. Your critical mind always was of great value. I want to thank Chao Sun for sharing his expertise as well. I also owe thanks to Jacco Snoeijer for his open-mindedness and analytical insight.

Although we never met in real life, I do want to thank Jon Chapman from the University of Oxford. Your mathematical expertise turned out to be crucial for solving an analytical problem. I also want to thank Jan Eijkel from the BIOS Lab-on-a-Chip research group for working together.

It is an honour for me that Cecile Cottin-Bizonne, Jan Eijkel, Steffen Hardt, Detlef Lohse and Hans Kuipers accepted the invitation to take place in my graduation committee. I am very grateful to you for doing that.

Anne Benneker and Jan van Nieuwkasteele, thank you for being my paranymphs! I have always enjoyed our moments of working together, and maybe even more all the nice (and amusing) conversations we had. I feel honoured that you both accepted this position and that you will be standing next to me during my defence.

Three persons in particular familiarised me with the world of scientific research. 
I want to thank Ludovic Carlier for coaching me during my bachelor assignment. Doing this in Paris was a valuable experience, also for my personal development. I also learned a lot from Elif Karatay and Claas Willem Visser. Thank you for your supervision during my master assignment. The experience I got during this period definitely formed a steppingstone towards my time as a $\mathrm{PhD}$ student. Luckily, we could continue our collaborations and discussions.

The project with Burcu Gümüşcü and Anne Benneker actually stood a bit aside from the main topic of my research. Nevertheless I liked to work on it, and we managed to transform an idea into a working device. Thank you for your willingness to join forces.

I want to thank all past and current members of the SFI group I worked with, if not mentioned already: Aura Visan, Can Aran, Cristina Franch, Damon Rafieian, Hanieh Bazyar, Ineke Pünt, Joeri de Valença, Khalid El Tayeb El Obied, Lidy Harryvan, Manas Manohar Mandalahalli, Mandakranta Ghosh, Pim Bullée, Roland Blanch Ojea, Vic van Dijk and Yali Zhang. The atmosphere in the SFI group was always very open and nice. The diversity of the group also created a very pleasant working environment. I will remember all our cookie breaks, group outings and dinners with great pleasure!

In addition to the SFI group, I also want to thank all members of the Membrane Science and Technology and of the Inorganic Membranes research groups for all the teamwork and all the nice (coffee) moments. I really enjoyed playing indoor soccer with the AC Membranes team. This definitely was no lost time, even though we lost many times!

I had the pleasure to supervise two students: Lisette Sprakel and Myrthe Bruning. The work you delivered is a clear sign of your competence. Thank you for working together and for contributing to this thesis.

Besides doing research, I got the opportunity to help teaching some courses. Thank you for that, Wim Brilman. I enjoyed doing it, and I will not forget that you gave me the chance to give my first lecture.

I believe that God has given everybody the task to exploit and to develop his or her talents. He is the source where our talents and abilities stem from. I have tried to do that as well as I could. Still, I am also convinced that this would not have been possible without having a solid base on which I could fall back on, every day again. I thank my family and friends for giving me a home and helping me to relax after work and during the weekends. I am grateful to my parents for having confidence in me, and for allowing me to pursue my interests, even though these were not always even clear. I also want to thank my parents(-in-law) for showing interest in my work.

Finally, and most importantly, I thank Corine for all her love and (mental) support during my years at the University of Twente. You motivated me to continue in tough periods, but you also forced me to have a good work-life balance. 
I thank God for giving us two beautiful children: Marieke and Gertjan. Raising children requires attention and energy, but this is outweighed by all their filial affection and dependency. It puts many things into perspective - indeed, also the work described in this thesis.

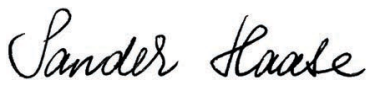





\section{About the author}

Andries Sander Haase was born on the 21st of February 1988 in Deventer, the Netherlands. In 2012, he married Corine Pluimers, with whom he has two children: Marieke and Gertjan. Since 2014, he has been a council member in the municipality of Rijssen-Holten.

He obtained his Gymnasium diploma cum laude at the Jacobus Fruytier scholengemeenschap in Apeldoorn in 2006. In the same year, he started his bachelor study in Chemistry at Utrecht University. In 2009 he obtained his BSc degree cum laude with a bachelor assignment on the characterisation of the three-dimensional structure of a protein using NMR spectroscopy at the Laboratoire des BioMolécules of the Uni-

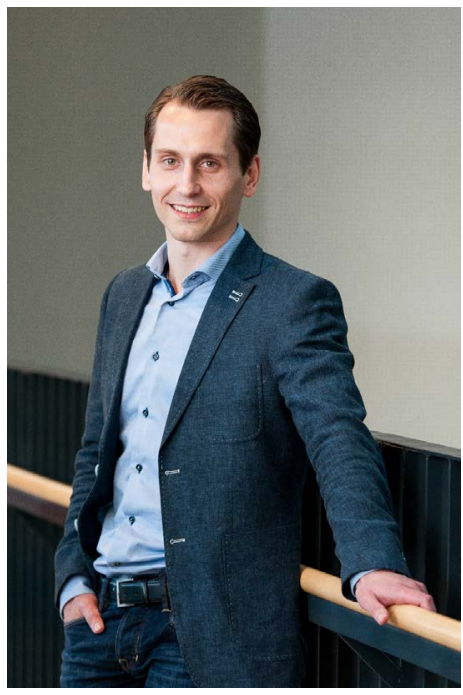
versité Pierre et Marie Curie (Paris IV).

In 2009, he continued his studies with a pre-master and master in Chemical Engineering (track Process Technology) at the University of Twente. He did his internship at Bayer Technology Services in Leverkusen, studying the dispersion of carbon nanotubes in low viscosity solvents. He performed his master assignment in the Soft matter, Fluidics and Interfaces research group on the transport of momentum and mass over a bubble mattress, which is a heterogeneously slippery superhydrophobic surface. In May 2012, he obtained his MSc degree cum laude. His thesis made him one of the finalists for the Shell Master Prize 2012.

In September 2012 he started as a PhD student in the Soft matter, Fluidics and Interfaces research group at the University of Twente, under the supervision of prof. dr. ir. Rob G.H. Lammertink. His research was directed towards transport over slippery surfaces, with the aim to contribute to a better understanding of various transport processes occurring at the nano- and microscale. He supervised one bachelor and one master assignment. In addition to doing research, he was a teaching assistant for the courses Heat and Mass Transfer and Introduction to Chemical Reactor Engineering.

In September 2016, he started working as Researcher Process Technology in the Research, Development \& Innovation department of AkzoNobel in Deventer. 



\section{Scientific output}

\section{List of publications}

submitted A.S. Haase, J.A. Wood, L.M.J. Sprakel and R.G.H. Lammertink, Inelastic non-Newtonian flow over heterogeneously slippery surfaces (2016).

2016 B. Gümüşcü*, A.S. Haase*, A.M. Benneker*, M.A. Hempenius, A. van den Berg, R.G.H. Lammertink and J.C.T. Eijkel, Desalination by electrodialysis using a stack of patterned ion- selective hydrogels on a microfluidic device, accepted for publication in Adv. Func. Mater. (2016).

2016 A.S. Haase, J.A. Wood, R.G.H. Lammertink and J.H. Snoeijer, Why bumpy is better: the role of the dissipation distribution in slip flow over a bubble mattress, Phys. Rev. Fluids 1, 054101 (2016).

2016 A.S. Haase and R.G.H. Lammertink, Heat and mass transfer over slippery, superhydrophobic surfaces, Phys. Fluids 28, 042002 (2016).

2015 A.S. Haase, S.J. Chapman, P.A. Tsai, D. Lohse and R.G.H. Lammertink, The Graetz-Nusselt problem extended to continuum flows with finite slip, J. Fluid Mech. 764, R3 (2015).

2013 A.S. Haase, E. Karatay, P.A. Tsai and R.G.H. Lammertink, Momentum and mass transport over a bubble mattress: the influence of interface geometry, Soft Matter 9, 8949 (2013).

2013 E. Karatay, A.S. Haase, C.W. Visser, C. Sun, D. Lohse, P.A. Tsai and R.G.H. Lammertink, Control of slippage with tunable bubble mattresses, P. Natl Acad. Sci. USA 110, 8422 (2013).

2012 L. Carlier, A.S. Haase, M.Y. Burgos Zepeda, E. Dassa and O. Lequin, The C-terminal domain of the Uup protein is a DNA-binding coiled coil motif, J. Struct. Biol. 180, 577 (2012).

2012 L. Carlier, A.S. Haase, M.Y. Burgos Zepeda, E. Dassa and O. Lequin, Secondary structure and NMR resonance assignments of the C-terminal DNA-binding domain of Uup protein, Biomol. NMR Assign. 6, 197 (2012). 


\section{Attended conferences and symposia}

2016 Burgers Symposium, Lunteren, the Netherlands

2015 68th meeting of the Division of Fluid Dynamics of the APS, Boston, USA

2015 IFPEN Microfluidics conference, Paris, France

2014 NPS14, Utrecht, the Netherlands

2014 FLOW14, Enschede, the Netherlands

2014 Physics@FOM, Veldhoven, the Netherlands

2014 Winterschool, Hirschegg, Austria

2013 International Soft Matter Conference, Rome, Italy

2013 European Congress of Chemical Engineering, The Hague, the Netherlands

\section{Supervised students}

2014 Lisette M.J. Sprakel, MSc assignment

Non-Newtonian flow on bubble mattresses

2014 Myrthe A. Bruning, BSc assignment

Momentum transport in liquid/liquid systems

\section{Attended courses}

2015 Professional Effectiveness

Novitijd and Centre for Training \& Development, University of Twente

2013 Numerical Methods for Chemical Engineers

Onderzoekschool Procestechnologie, Eindhoven University of Technology

2012 Methodologies in the Engineering Sciences

Twente Graduate School, University of Twente

2012 Advanced Fluid Mechanics

J.M. Burgerscentrum, University of Twente

2012 Cleanroom courses

$\mathrm{MESA}^{+}$NanoLab, University of Twente 





\section{SLIPPERY INTERFACES}

Some surfaces exhibit wall slip, meaning that a flowing fluid has a velocity relative to that surface. This leads to increased advection in the direct vicinity of the wall. As this directly affects the rate of transport at larger scales, slippery surfaces have a potentially important application in enhancing interfacial transport. The knowledge obtained through fundamental investigations of these micro- and nanoscale transport phenomena is therefore of high importance.

The aim of this thesis is to extend the current knowledge on transport near slippery and/or heterogeneous interfaces. For that purpose, various experimental, analytical and numerical studies have been performed. The following topics have been investigated:

- the influence of hydrodynamic slip on momentum and mass transfer over superhydrophobic surfaces, focusing on a so-called bubble mattress;

- the extension of a classical forcedconvection problem to homogeneously and heterogeneously slippery surfaces, describing heat and mass transfer near these surfaces;

- ion transport near heterogeneous. charge-selective interfaces in a microfluidic desalination device.
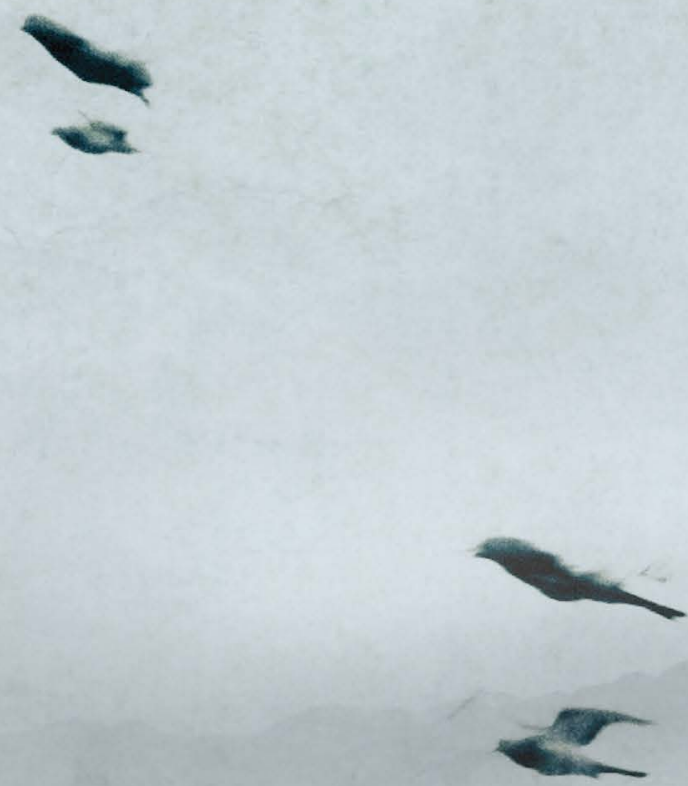